\title{
An investigation into the ecology and evolution of caterpillar eyespots
}

by

Thomas John Hossie

A thesis submitted to the Faculty of Graduate and Postdoctoral Affairs in partial fulfillment of the requirements for the degree of

Doctor of Philosophy

in

Biology

Carleton University

Ottawa, Ontario

(C) 2014, Thomas John Hossie 


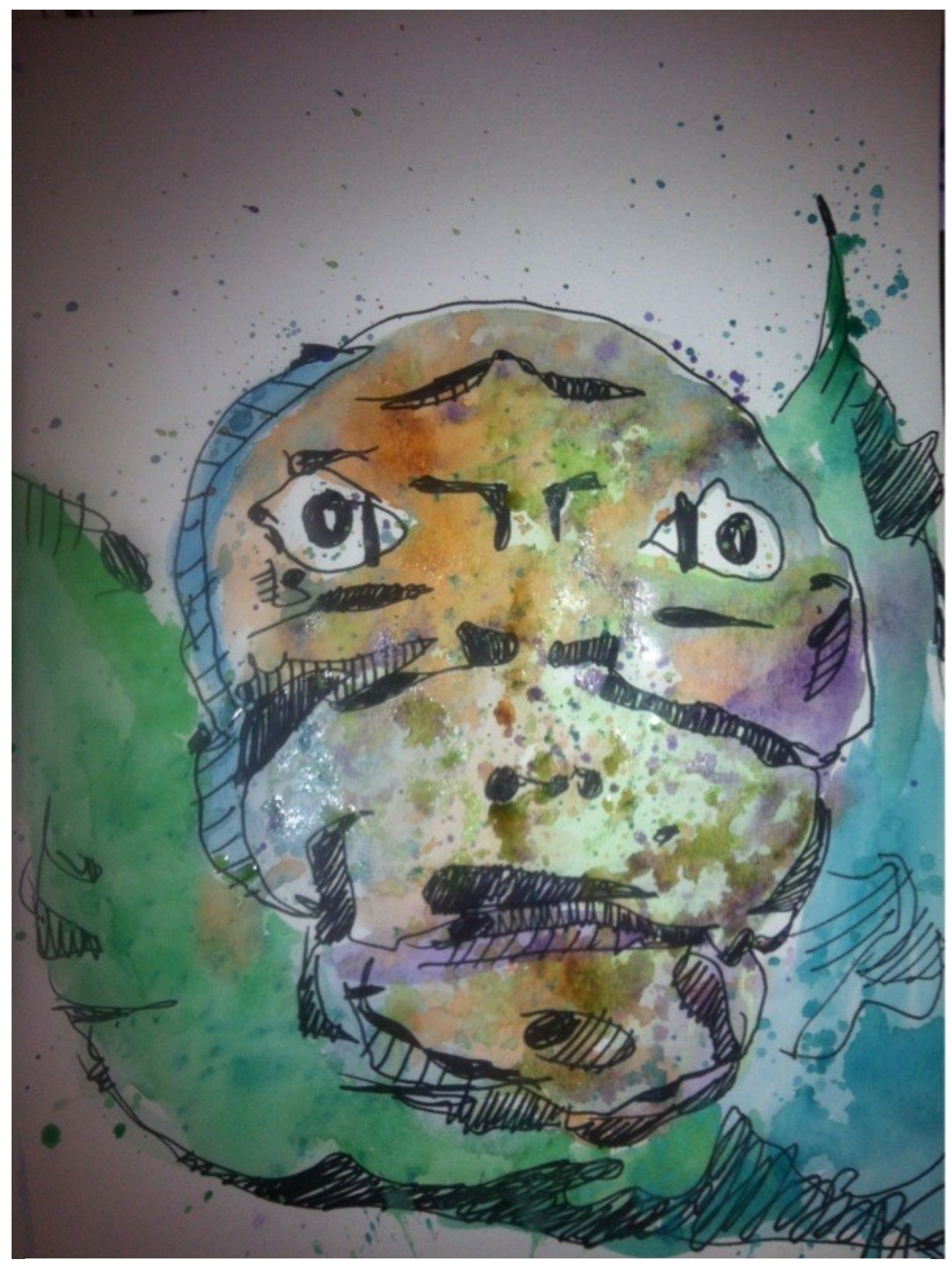

"Bad Portrait" painted by artist Mandy Stobo based on a photo of a Papilio canadensis caterpillar taken by Thomas Hossie 


\section{Abstract}

Eyespots are conspicuous circular patterns on the body of an animal which superficially resemble vertebrate eyes. These odd markings have long captured the interest of biologists and naturalists alike, many of whom have suggested that eyespots mimic the real eyes of dangerous animals and thereby protect prey species from their attackers. Eyespots are particularly widespread and diverse in lepidopteran caterpillars, and these caterpillars with eyespots are typically assumed to be snake-mimics. Yet a dearth of empirical investigation has left us without evidence that eyespots can protect caterpillars, or the ability to substantiate our subjective belief that these caterpillars are mimicking snakes. Using a combination of field and lab experiments my research provides robust empirical evidence demonstrating that eyespots are an adaptation in caterpillars, but reveals that other factors, including body colour and body size, influence their efficacy as a deterrent. Moreover, my work reveals that the defensive posture, which many caterpillars with eyespots adopt when harassed, also provides protection independent from the possession of eyespots. Using an objective measure of mimetic fidelity I showed that this defensive posture is likely a form of behavioural mimicry which increases the caterpillar's resemblance to viperine snakes when viewed dorsally. Caterpillars from three distinct families showed analogous behavioural mimicry, suggesting strong selection for convergent phenotypes across lepidopteran larvae. Finally I provide an explanation for why some caterpillars have eyespots, and others do not. I show that body size critically influences the evolution of eyespots using a phylogenetically-controlled analysis of Macroglossinae (Sphingidae) caterpillar traits. Using field and lab experiments I reveal that the protective value of eyespots to caterpillars is in fact size-dependent: eyespots are only intimidating to attackers when on large-bodied prey that are already easy to detect, yet eyespots hinder small prey by making them easy to detect and failing to intimidate attackers. I argue that caterpillar eyespots have evolved to serve a mimetic function and were generated and are currently maintained by the innate fear of predator eyes harbored to varying degrees by numerous species of insect-eating birds. 


\section{Acknowledgements}

I would like to extend my sincere gratitude to my supervisor Tom Sherratt. He has been an excellent mentor throughout my degree. When I started my doctorate I did not know what the topic of my dissertation would be, but he allowed me the time I needed to develop the research program presented here. Tom gave me the freedom to dictate the direction of my research though all stages, but always provided the critical feedback required to make these projects successful. I am also grateful for his support of the several rewarding opportunities I have had throughout my degree including a month-long field trip to Costa Rica, and numerous conferences (Quebec City, Banff, Ottawa, Newcastle (UK), and Ventura). His feedback on the work included below resulted in substantial improvements and it has been a pleasure having him as a supervisor.

My committee members Gabriel Blouin-Demers and Jayne Yack have been enthusiastic and supportive throughout my degree. Jayne in particular pushed me contact Dan Janzen and arrange a trip to Costa Rica which would end up being a highlight of my $\mathrm{PhD}$ experience. Dan Janzen and Winnie Hallwachs encouraged my travel to the Área Concervación Guanacaste in Costa Rica, and facilitated my visits to the various collecting stations during my stay. They allowed me to photograph and conduct behaviour tests on the numerous caterpillars at the collecting stations, which introduced me to a diversity of species I would not have otherwise experienced. Dan in particular has provided me with valuable insight into caterpillar eyespots that could not have been gleaned elsewhere. I thank my collaborators John Skelhorn, Akito Kawahara, Jesse Breinholt, Dan Janzen, and Winnie Hallwachs; each of their contributions to 
this thesis were pieces that I was unable to add myself and have improved the calibre of this research.

I would like to thank members of the Sherratt and Gorelick lab for their support and critical feedback, especially Chris Hassall and Richard Webster. Lee Gutowsky and I began our doctorates in Biology at the same time, and lived together for the first year and a half of our degree. I have greatly valued his friendship during my time in Ottawa. Similarly, I have appreciated the company of friends and colleagues Jake Brownscombe, Wayne Knee, Graham Raby, and Jason Thiem.

Much of my fieldwork was conducted on land owned by Bill and Eleanor Hossie. They, along with my parents Mary Alice and James Hossie, unwaveringly encouraged my pursuits in biology and were always interested to hear about my research. I attribute much of my interest and appreciation for the natural world to my mother.

Finally, I thank my partner Holly Kienzle. She gave up steady work as a teacher and proximity to her friends and family to come join me in Ottawa while I finished my degree. I know it has not always been easy for her, and I appreciate her continued love and support. 


\section{Co-authorship Statements}

Several pieces of this thesis document have been published including data chapters 2-5. Chapter 6 is soon to be submitted. Dr. Tom Sherratt was a coauthor on data chapters 2 and 3 which were published in Animal Behaviour (Hossie and Sherratt 2012, 2013), and chapter 4 which was published in Current Zoology (Hossie and Sherratt 2014). Specifically, Dr. Sherratt helped with the experimental design, analysis, and writing of this work. Chapter 5 was a natural history paper that was co-authored by Dr. Winnie Hallwachs, Dr. Daniel Janzen and Dr. Sherratt (Hossie et al 2013). Dr. Hallwachs and Dr. Janzen facilitated the observation that led to the description, provided great insight into the life history and behaviour of the taxa described, contributed photos, and assisted with writing. Dr. Sherratt gave the initial push to publish the description and contributed to the writing of the manuscript. Chapter 6 was co-authored by Dr. John Skelhorn, Dr. Akito Kawahara, Dr. Jesse Breinholt and Dr. Tom Sherratt. Dr. Skelhorn and I collaboratively designed the chick experiment portion of this work, and the actual chick experiment itself was conducted by Dr. Skelhorn. Dr. Kawahara and Dr. Breinholt built the phylogeny that was used in this chapter. Dr. Skelhorn, Dr. Kawahara, Dr. Breinholt, and Dr. Sherratt assisted with the writing of the manuscript. For all data chapters included in this thesis I conducted all of the analyses (excluding phylogeny estimation in Ch. 6), generated all the figures, and drafted all initial and final manuscripts.

Chapters 2 and 3 are reprinted herein with the permission of Elsevier. Chapter 4 is reprinted with the permission of Current Zoology, and chapter 5 is reprinted in accordance with the policies of Taylor \& Francis. 


\section{Table of Contents}

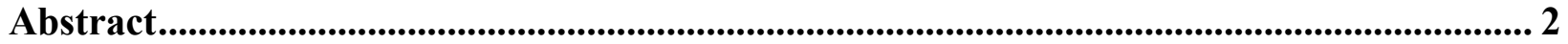

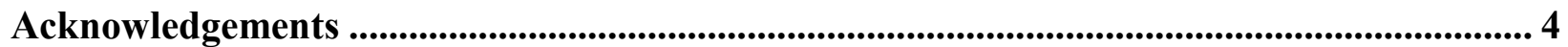

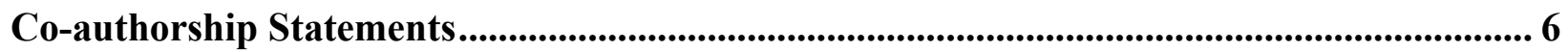

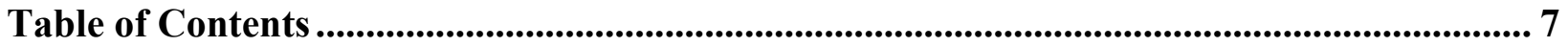

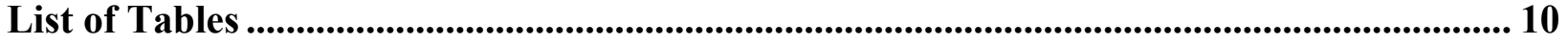

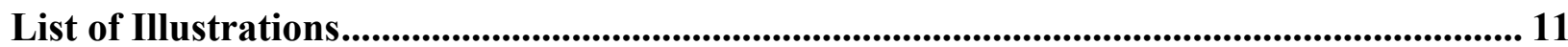

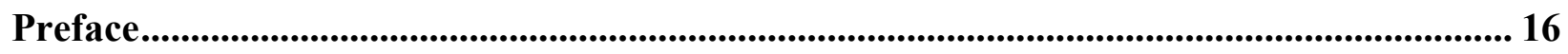

1 Chapter: A general introduction to topics covered in the thesis .................................... 19

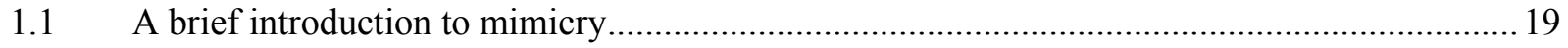

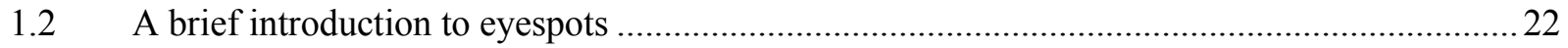

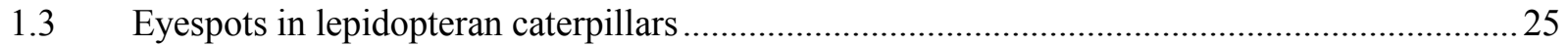

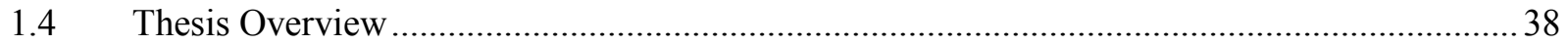

2 Chapter: Eyespots interact with body colour to protect caterpillar-like prey from avian

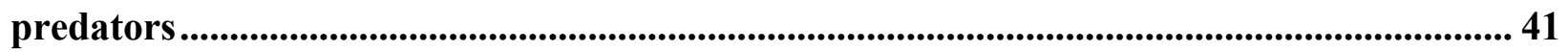

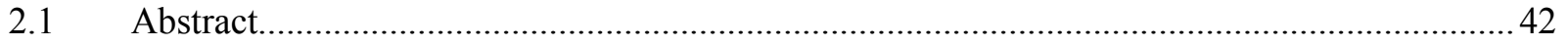

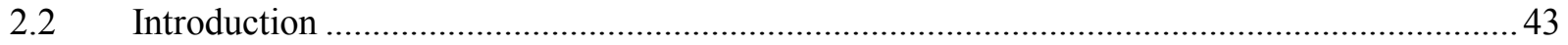

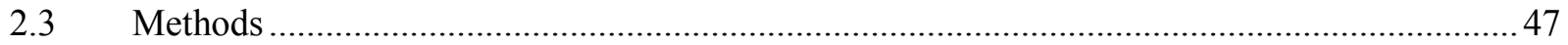

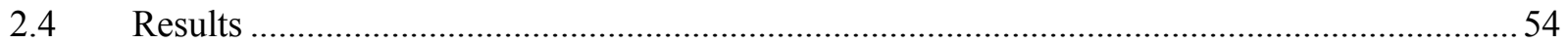

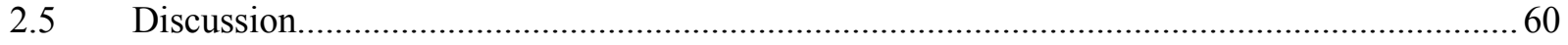

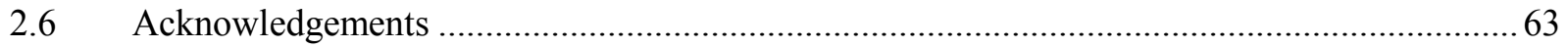

3 Chapter: Defensive posture and eyespots deter avian predators from attacking

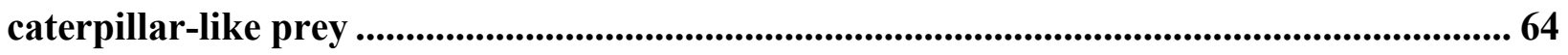

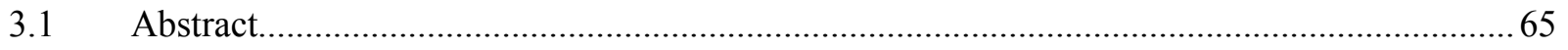

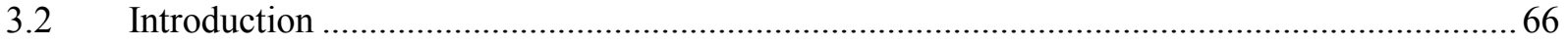




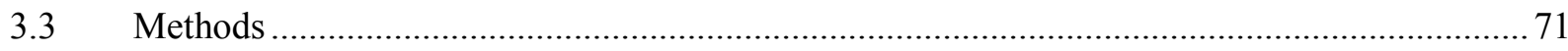

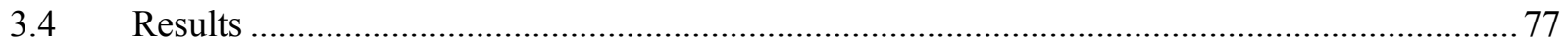

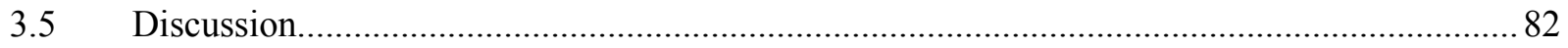

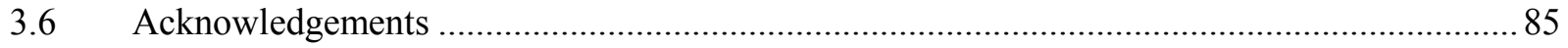

4 Chapter: Does defensive posture increase mimetic fidelity of caterpillars with eyespots to their putative snake mimics? ........................................................................................................ 86

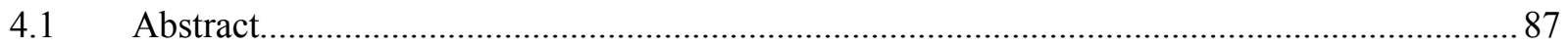

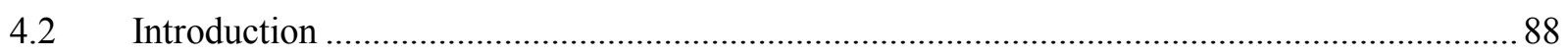

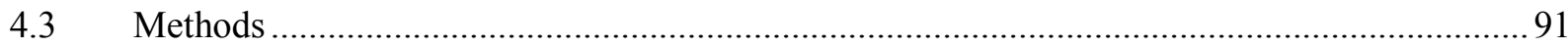

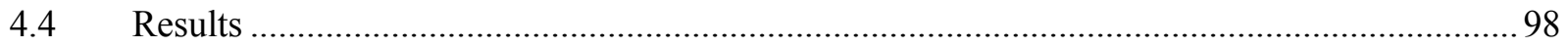

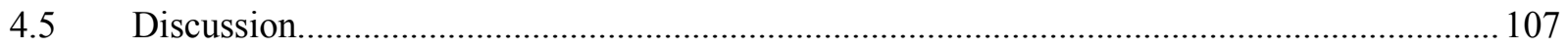

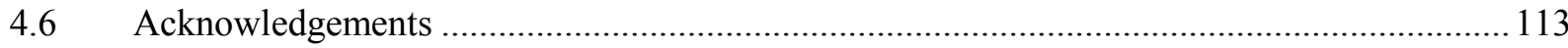

5 Chapter: An eyespot that "blinks": an open and shut case of eye mimicry in Eumorpha caterpillars (Lepidoptera: Sphingidae) ..................................................................... 114

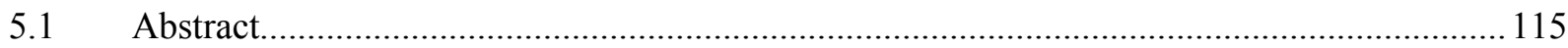

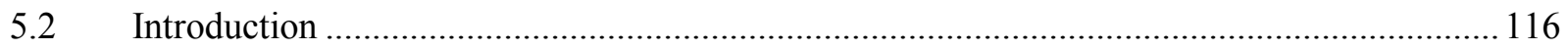

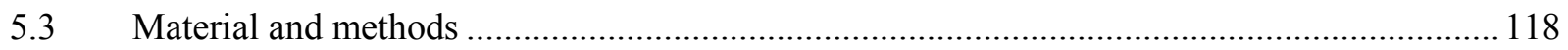

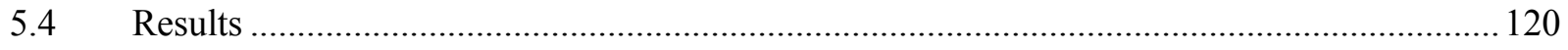

5.4.1 Description of Eumorpha phorbas caterpillar............................................................ 120

5.4.2 Description of Eumorpha labruscae caterpillar ........................................................... 124

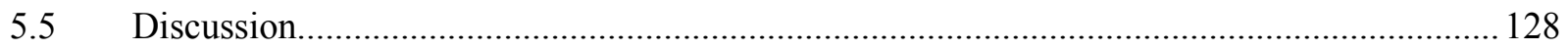

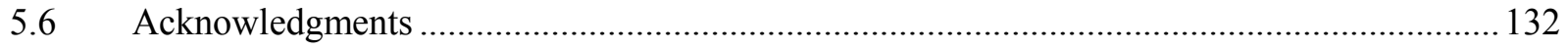

6 Chapter: Body size affects the evolution of eyespots in caterpillars ........................... 133

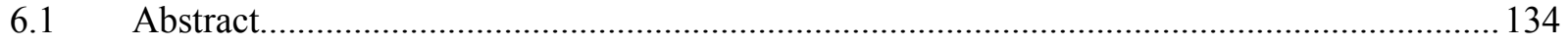

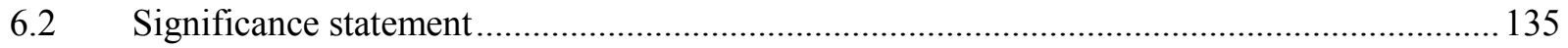

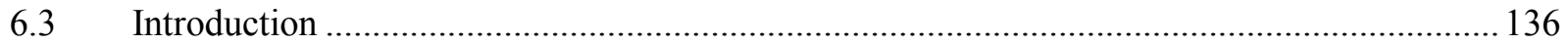




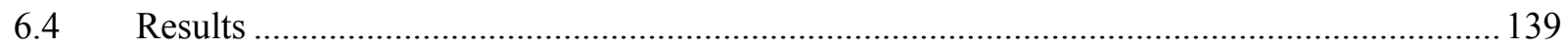

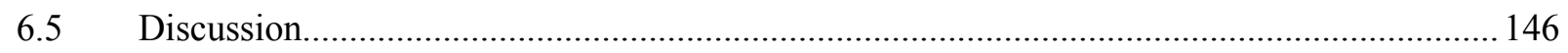

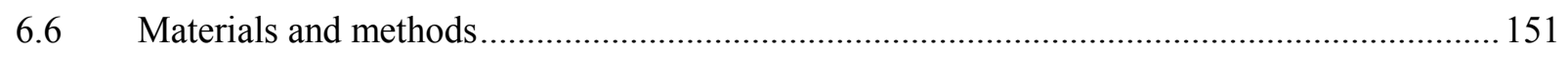

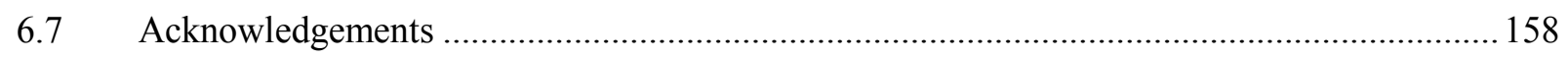

7 Chapter: General conclusions and future work................................................... 159

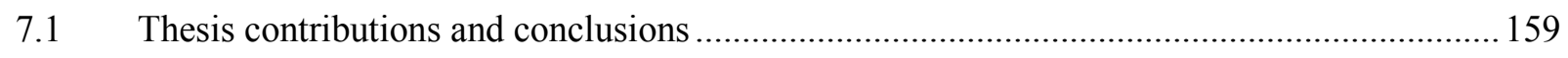

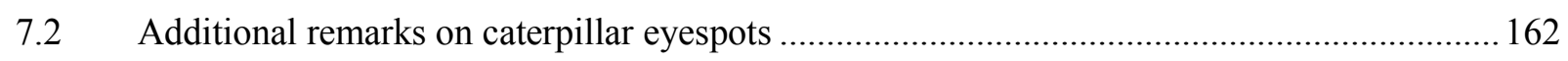

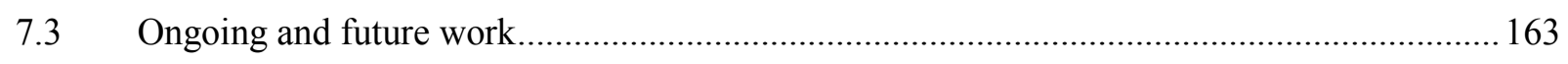

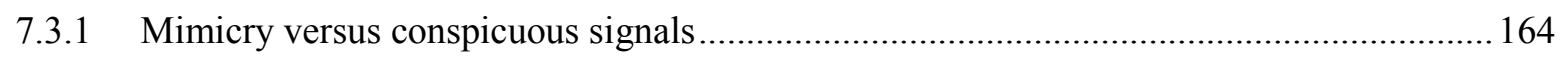

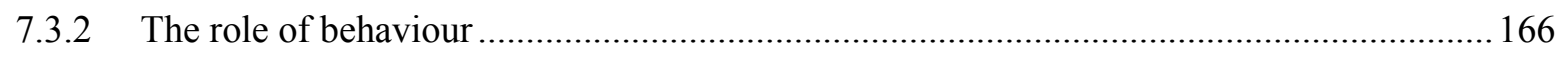

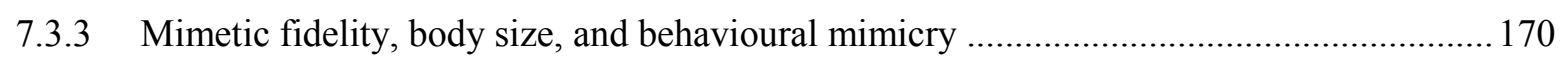

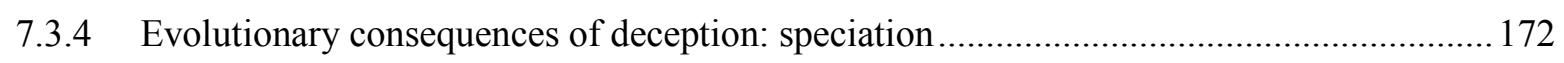

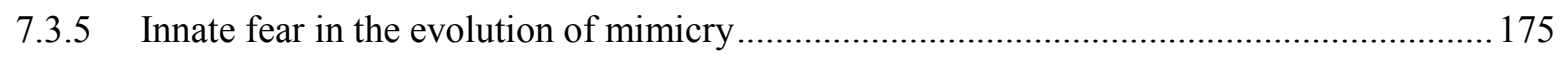

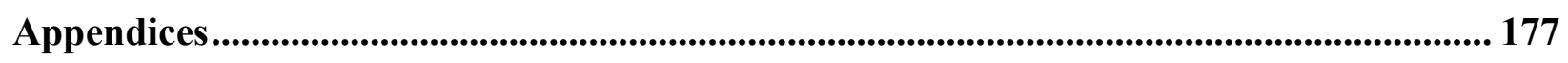

Appendix A Blog posts from “Caterpillar Eyespots” highlighting Moss' descriptions of snake-mimic

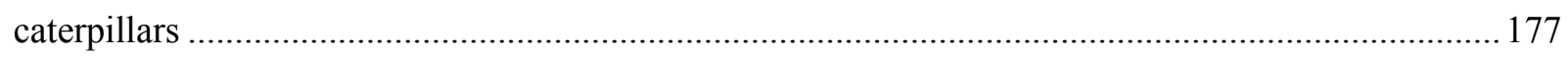

A.1 Moss' account of Hemeroplanes larvae in Para .......................................................... 177

A.2 Moss' account of another snake-mimic: Madoryx plutonius ......................................... 182

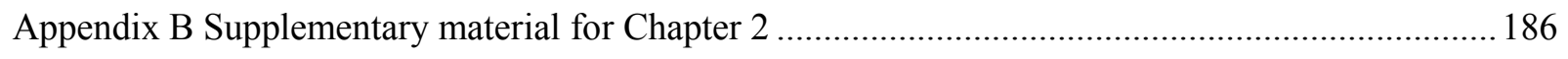

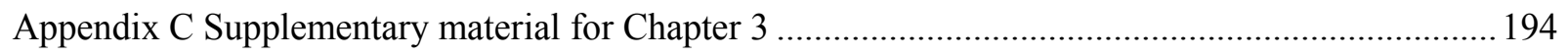

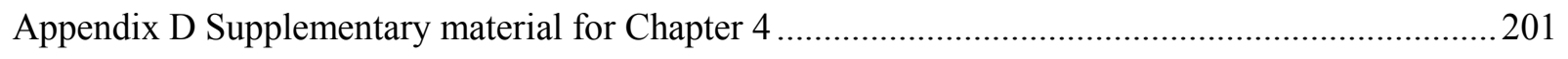

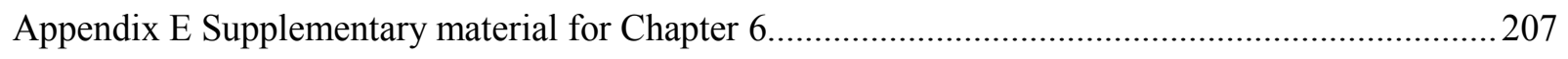

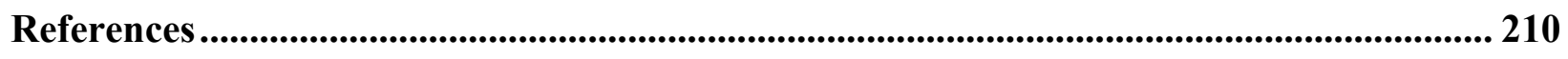




\section{List of Tables}

Table 1-1 Outline of possible nonmutually exclusive functions of eyespots in lepidopteran caterpillars and the relevant literature

Table 2-1: Results from fitting Cox proportional hazards regression model of pastry caterpillar survival stratified by location and tree species

Table 3-1: Results from fitting Cox proportional hazards regression model of pastry caterpillar survival stratified by location and tree species. 78

Table 4-1: The position of landmarks for geometric morphometric comparison of 'eyespots' and body shape of caterpillars with eyespots and the corresponding landmark positions on snakes.. 95 Table 4-2: Results from matched-pairs t-tests comparing the shape of caterpillars in their rest and defensive postures for each of four shape variables (i.e. principal components). 103 


\section{List of Illustrations}

Figure 0-1: A page out of my field notebook from a trip to Peru in 2007 depicting a final instar Hemeroplanes sp. caterpillar. 16

Figure 1-1: Final instar Sphingidae caterpillars illustrating what August Weismann's distinction between "eye-spots" (panels A, B) and "ring-spots" (panels C, D), which he considered to have distinct functions. A) Deilephila porcellus, photo by "NaturKamera"; B) D. elpenor, photo by Joachim Baecker; C) Hyles gallii, photo by "Hectonichus". H. gallii caterpillars also have a black colour variant; D) Hyles euphobiae, photo by Daniel Schwen. All four photos accessed from Wikipedia Commons and used freely as per their Creative Commons licence. 28

Figure 1-2 Final instar Papilio genus (Papilionidae) caterpillars in their defensive posture, everting their osmererium from behind their head. Though this structure likely functions to deter insect or parasitoids, and emits volatile chemicals. Many have argued that the osmeterium has been co-opted to facilitate snake mimicry in at least some Papilio species. This is because the structure resembles a snake's tongue because of its orange-red colour, location in relation to other eye- and face-like features, and the fact that it is forked. A) Papilio canadensis; B) Papilio cresphontes, a bird-dropping mimic which appears to retain comparable defensive posture and vestigial eyespots. Both photos by T. Hossie in Kemptville, ON, summer of 2011, and 2013 respectively.

Figure 2-1: Examples of pecked pastry caterpillars from each of the four treatments used in this

experiment. (a) Solid-no eyespots; (b) Countershaded-no eyespots; (c) Solid-eyespots; 50 Figure 2-2: (a) Results from pairwise Cox proportional hazard regression comparisons of pastry caterpillar survival stratified by tree species and location, and clustered by the tree to which 
pastry caterpillars were pinned. Eyespot (no eyespots versus eyespots present) and colour (solid green versus countershaded) treatments were arranged in a $2 \times 2$ factorial design. (b) Results from the same analyses conducted using only those pastry caterpillars pinned to Populus tremuloides (primary host of Papilio canadensis; Scriber et al. 1995). In both panels, arrow width is proportional to the relevant Wald statistic and points towards the treatment with lower hazard rate. Solid arrows are significant at $P<0.05$. 57 Figure 2-3: Box plot depicting the number and location of peck attacks on pastry caterpillars deployed in the field after controlling for the lack of independence of multiple pecks on a given individual prey item. White boxes: no-eyespot treatment; grey boxes: eyespot treatment. The corrected median number of pecks is depicted by the bar inside the box, hinges indicate the $25 \%$ and $75 \%$ quartiles, and whiskers extend to 1.5 times the interquartile range, with values outside of this range depicted as open circles. 59

Figure 3-1: (a) Penultimate instar of a Costa Rican Hemeroplanes triptolemus (Sphingidae) caterpillar displaying its defensive posture; what appears to be the dorsal side of the snake head is in fact the ventral side of the caterpillar thorax. Photo is from Janzen and Hallwachs (2014), specimen number 03-SRNP-11366 (used with permission). (b) Ultimate instar Papilio canadensis caterpillar in resting posture, and (c) ultimate instar Papilio canadensis caterpillar in defensive posture (photos by TJH) 70 Figure 3-2: Examples of pecked pastry caterpillars from each of the four treatments used in this experiment. (a) no eyespots-resting posture; (b) no eyespots-defensive posture; (c) eyespotsresting posture; (d) eyespots-defensive posture. 73 Figure 3-3: Results from pairwise Cox proportional hazard regression comparisons of pastry caterpillar survival stratified by tree species and location, and clustered by the tree to which 
pastry caterpillars were pinned. Eyespot (i.e., no eyespots versus eyespots present) and HeadShape (i.e., resting posture versus defensive posture) treatments were arranged in a $2 \times 2$ factorial design. Arrow width is proportional to the relevant Wald statistic and points towards the treatment with lower hazard rate. Solid arrows are significant at $\mathrm{P}<0.05$. 79

Figure 3-4: Box plot depicting the median number and location of peck attacks on pastry caterpillars deployed in the field after controlling for the lack of independence of multiple pecks on a given individual prey item. White boxes: no-eyespot treatment; grey boxes: eyespot treatment. The corrected median number of pecks is depicted by the bar inside the box, hinges indicate the $25 \%$ and $75 \%$ quartiles, and whiskers extend to 1.5 times the interquartile range, with values outside of this range depicted as open circles

Figure 4-1: Landmark placements for caterpillars and snakes in their dorsal and lateral views. A) Papilio canandensis (Papilionidae) caterpillar in defensive posture, dorsal view; B) Leptodeira annulata (Colubridae) snake, dorsal view; C) P. canadensis caterpillar defensive posture, lateral view; D) L. annulata, lateral view. 96

Figure 4-2: Vector plots illustrating the shape change described by principal components (PCs) 1 and 2 for both the dorsal and lateral view. Vectors point in the direction of an increasing PC value.

Figure 4-3: The morphospace occupied by caterpillars with eyespots in their resting and defensive postures as compared to Colubridae and Viperidae snakes. Only one Boidae snake was measured and its location is demarked with an $\mathrm{X}$. 101

Figure 4-4: Posterior probabilities for the classification of caterpillars from 14 species as a caterpillar (black), colubrid snake (grey), or viperid snake (hatched) when in their resting and 
defensive posture. Posterior probabilities shown here were extracted from a linear discriminant analysis using only dorsal features that changed with posture (i.e., dorsal PC2 only). 106

Figure 5-1: Lateral view of Eumorpha phorbas caterpillar during the penultimate (A) and ultimate (B) instars.

Figure 5-2: Close-up views of Eumorpha phorbas caterpillars. (A) Dorsal view of the anterior body segments, (B) dorso-lateral view of posterior eyespot which replaces the anal horn present in the penultimate instar. 123

Figure 5-3: Lateral view of Eumorpha labruscae caterpillar during the penultimate (A) and ultimate (B) instars. 126

Figure 5-4: Close-up views of Eumorpha labruscae caterpillar. (A) Lateral view of the anterior body segments, (B) dorsal view of posterior eyespot that which replaces the anal horn present in the penultimate instar.

Figure 6-1: Phylogeny of Macroglossinae (Sphingidae) species included in the analysis. Black symbols indicate the species whose caterpillars possess eyespots and the number inside indicates the caterpillar's maximum body length $(\mathrm{cm})$ at the larva's final instar. 140 Figure 6-2: Results from pairwise Cox proportional hazard regression comparisons of pastry caterpillar survival in the field experiment. Eyespot (i.e. no eyespots versus eyespots present) and body size (i.e. $2 \mathrm{~cm}$ versus $4 \mathrm{~cm}$ in length) treatments were arranged in a $2 \times 2$ factorial design. Arrow width is proportional to the relevant Wald statistic and point towards the treatment with lower hazard rate. Solid arrows are significant at $\mathrm{P}<0.05$. 142

Figure 6-3: Results from the lab experiment using domestic chicks. Bars represent the latency to attack broken up into search time (i.e., time to first monocular inspection), and inspection time (i.e., time from first inspection to attack). Ten chicks were exposed to each treatment. Vertical 
lines on each horizontal bar represent the estimated standard error (SEM). The thickened vertical lines represent the SEM for overall latency to attack. 145

Figure 7-1: Experimental set up for a failed experiment in 2011 which set out to investigate bird responses to caterpillar eyespots and anti-predator behaviour (arranged as a 2x2 factorial). A) The feeding table during training phase. Live "superworms" (Zophobas morio larvae) tethered to screws using monofilament fishing line and hair-removal wax, and black sunflower seeds added to draw in birds and acclimate them to feeding on the table. B) The table and video camera set up in in field as viewed from the blind 20m away. C) Close-up of a tethered superworm D) Close-up of a tethered silkworm (Bombyx mori larvae), E) Tethered silkworm upon which eyespots were painted used as a preliminary test of the system. 168

Figure 7-2: Photos from the prototyping process for a caterpillar model that could move upon attack by wild birds. A) The dynamic arm with spring-loaded release motion. B) A pressure sensitive signal receiver which would be triggered by a bird peck. C) A solenoid actuator which would activate and reset the dynamic arm. D) A schematic of how the model would be deployed in the field. 169 


\section{Preface}

The most erroneous stories are those we think we know best—and therefore never scrutinize or question.

\section{— Stephen Jay Gould}

Full House: The Spread of Excellence from Plato to Darwin (1997), 57.

Ask any naturalist and they'll tell you, eyespots protect prey animals because they resemble the eyes of a predator or other dangerous animal and thereby startle or intimidate would-be attackers; but how do we know this? How can we know that these markings, which look like eyes to us, similarly resemble eyes to birds or other animals? More fundamentally, what evidence do we have to even suggest that these markings deter predators from their attack?

In February of 2007 I took part in a tropical field course set in the Peruvian Amazon. This gave me the chance to observe and study tropical ecology first hand. Knowing that I may never get a chance to return I took every chance I could to see more. Halfway through our trip while hiking as a group one of my classmates was startled by what appeared to be an arboreal viper reaching from the branches. In reality, it was a caterpillar. Specifically, this was Hemeroplanes ornatus (or possibly $H$. triptolemus as they are difficult to distinguish as caterpillars), a spectacular sight - these caterpillars are among the best of the snake mimics. At rest they are well concealed, the dorsal side of their body resembling tree bark, but when discovered they reach out from the foliage

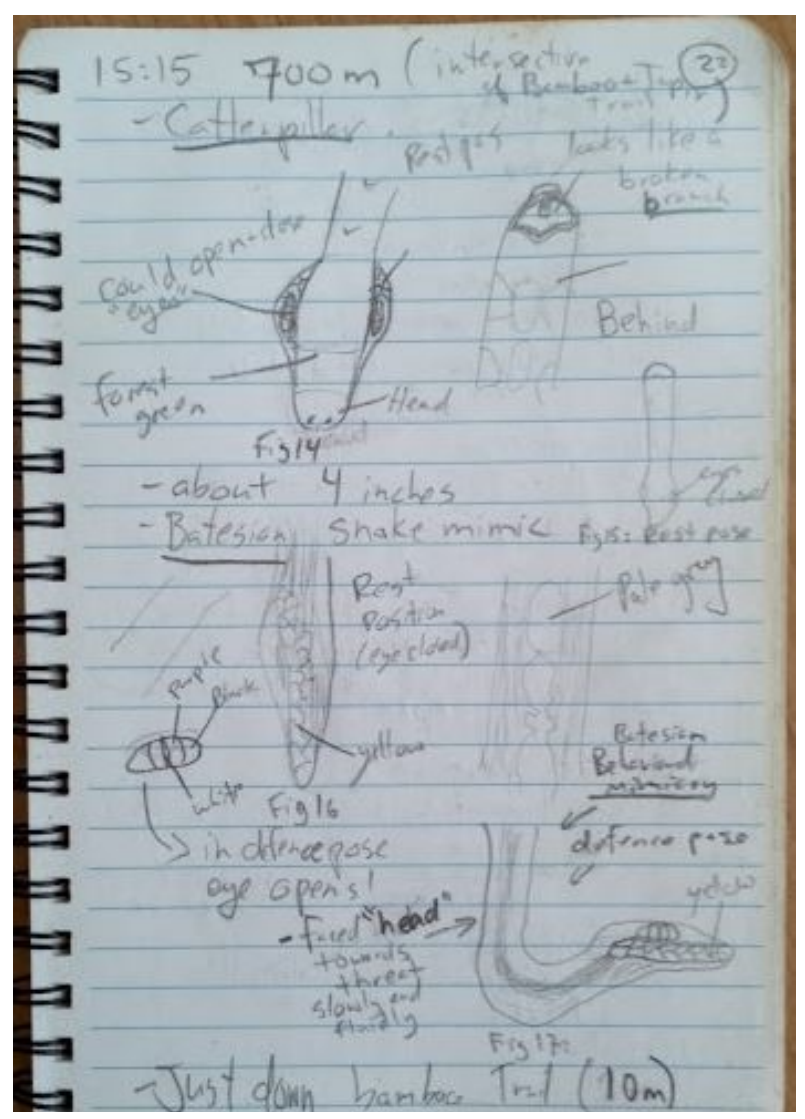

Figure 0-1: A page out of my field notebook from a trip to Peru in 2007 depicting a final instar

Hemeroplanes sp. caterpillar. 
inflating their anterior body segments into a viperine-shaped head. In a manner unlike any other species I have encountered, these caterpillars display ventral side up. Only upon inflation of the anterior segments do the eyespots become visible. The caterpillar throws itself backward and sways this false head towards the perceived threat, not in a jerky thrash one might expect from a large bodied caterpillar, but in a smooth, sinuous, fluid motion.

Even with my limited knowledge of mimicry at the time I knew of Bates and Müller's theories, and knew that Bates formulated his ideas while traveling in the Amazon (though primarily based on his observations of adult butterflies). It wouldn't be until much later (early 2010) that I would realize the Bates likely encountered this same species of caterpillar during his own travels from 1848-1859. In fact, in the very paper where Bates first put forward his theory of adaptive resemblance, now known as Batesian mimicry, he included a description of his encounter:

"The most extraordinary instance of imitation I ever met with was that of a very large Caterpillar, which stretched itself from amidst the foliage of a tree which I was one day examining, and startled me by its resemblance to a small Snake. The first three segments behind the head were dilatable at the will of the insect, and had on each side a large black pupillated spot, which resembled the eye of the reptile: it was a poisonous or viperine species mimicked, and not an innocuous or colubrine Snake; this was proved by the imitation of keeled scales on the crown, which was produced by the recumbent feet, as the Caterpillar threw itself backwards. The Rev. Joseph Greene, to whom I gave a description, supposes the insect to have belonged to the family Notodontida?, many of which have the habit of thus bending themselves. I carried off the Caterpillar, and alarmed every one in the village where I was then living, to whom I showed it. It unfortunately died before reaching the adult state."

-Henry Walter Bates, 1862, p509

Clearly Bates saw this as an important example of adaptive resemblance. There are a number of Notodonta caterpillars that have eyespots, yet his description points to a Hemeroplanes 
caterpillar. Specifically, he highlights that the caterpillar threw itself backwards such that the recumbent feet (i.e., the true legs) were visible and, to my knowledge, Hemeroplanes are among the only species that display this way (i.e., ventral side up). Furthermore, no New World caterpillar of which I'm aware is as convincingly viper-like when threatened.

Like Bates, I did not need convincing that this caterpillar was mimicking a snake. In fact, I have not encountered a single skeptic of the view that this caterpillar is a snake-mimic once they have seen photos or video footage. Clearly this caterpillar is a snake mimic, and clearly those large black pupillated spots are mimicking eyes. Was this judgment subjective? Perhaps. But what else could possibly explain the appearance and behaviour of this caterpillar? For decades this line of reasoning was the principal evidence put forth to support the widely-held contention that those caterpillars possessing eyespots, including but not limited to Hemeroplanes, mimicked snakes and were thereby protected from insect-eating birds. As a scientist however, subjective assessments aren't good enough. Objective empirical evidence is required to justify our faith in our ideas, irrespective of how obvious they seem or how widely held the belief. Only through this pursuit can we ever hope to fully appreciate the complexity of the world in which we live.

Thomas Hossie

Ottawa, 2014 


\section{Chapter: A general introduction to topics covered in the thesis}

\subsection{A brief introduction to mimicry}

Predation is an important source of mortality in many species, as evidenced by the multitude of anti-predator adaptations we observe in the natural world. Yet, despite intense study of predator-prey systems we still lack a synthetic framework from which we can resolve general trends in the evolution of specific anti-predator traits. For instance, the conditions favouring the evolution of Batesian mimicry over other seemingly equivalent predator defences (e.g., crypsis, aposematism, spinescence) remain unclear. With the plethora of extremely cryptic prey, it is not immediately obvious why natural selection would favour alternative defences such as mimicry over simply improved camouflage. Careful examination of the conditions where anti-predator defences differ among closely related species, or are analogous among distantly related groups, can provide the critical insight required to understand when and why such changes occur. In determining those features of predator-prey systems that consistently co-vary with specific antipredator defences we build a foundation upon which theoreticians and empiricists can develop a more complete understanding of why prey have the defences they do.

Mimicry is an intrinsically interesting defence strategy. Natural selection has produced some astonishing cases of adaptive resemblance, with many cases only now being fully appreciated (e.g., Gluckman \& Mundy, 2013; Nelson \& Jackson, 2009; O’Hanlon, Holwell, \& Herberstein, 2014). In its original formulation, mimicry represents a form of adaptive resemblance where an edible species gains protection from predators by resembling an inedible or noxious species (Bates 1862). Here, the predator learns to associate noxiousness with a given prey species (the model) through sampling and avoid attacking these prey in subsequent encounters. The mimic benefits through resembling the model species to the degree that the 
predator's learned aversion to the model is generalized to also include unrelated, but edible, mimics. Bates developed this idea of mimicry after spending 11 years in South America. Being a keen entomologist and taxonomist, he noticed a number of puzzling phenomena when collecting and cataloguing Heliconidae butterflies in the Amazon. Occasionally, he would catch Pieridae butterflies which looked and behaved so much like the co-occurring Heliconidae species that he could not discriminate between them on the wing. Although Heliconidae species were both conspicuous and slow flying, Bates never observed them being pursued by birds or other enemies, whereas those Pieridae that did not resemble Heliconidae were frequently pursued. In total Bates would catalogue some 14 species of Pieridae, along with additional species from Castiniidae and Papilionidae, which imitated the wing patterns and flight behaviour of cooccurring Heliconidae butterflies (Bates 1862). These observations would eventually culminate into his development of the theory that now bears his name.

As one of Darwin's contemporaries, the idea of protection through imitation came at an important time during the development of Darwin's the theory of evolution by natural selection. Bates explicitly formulated his theory of adaptive resemblance in the context of evolution by natural selection and stated: "I believe the case offers a most beautiful proof of the truth of the theory of natural selection" (H. Bates, 1862, p513). Darwin clearly realized the importance of this work, telling Bates in a letter that "In my opinion it is one of the most remarkable \& admirable papers I ever read in my life." (Letter to Bates, Nov. 20 1862), and congratulating him on his great work that he was sure would have lasting value.

Since its original formation by Bates, mimicry theory has been expanded to include several distinct forms of adaptive resemblance. Fritz Müller, suggested that mimicry could also evolve in cases where two noxious species both benefit from resembling each other (Müller 
1879). The key benefit he suggested is that co-mimicry reduces the cost of predator education, as fewer individuals of each species need to be killed by a naïve predator before the predator learns to associate a given signal with unpalatability. This form of mimicry is now termed Müllerian mimicry, but did not initially gain wide acceptance (see Sherratt 2008). Important empirical validations would come later and show that unrelated taxa that are equally unpalatable, through different means, indeed share signals to reduce the cost of predator education (e.g., Mallet et al. 1989; Ritland \& Brower 1991; Kapan 2001). The degree and direction of asymmetries in the noxiousness of model and mimics are sometimes ambiguous, which can make classifying a given mimicry system as Batesian or Müllerian difficult. This has led to the development of quasiBatesian mimicry theory (Speed 1993; Mallet \& Joron 1999; Speed \& Turner 1999). Another widely recognized form of mimicry is "aggressive mimicry" where adaptive resemblance is used not to avoid predators, but instead to deceive receivers and thereby improve access to their resources (Endler 1981; Saul-gershenz \& Millar 2006; O’Hanlon et al. 2014).

Of interest to evolutionary biologists is how defences like Batesian mimicry can arise from rarity. In true Darwinian form, Bates believed that this proceeded in small incremental steps (Bates 1862). Yet, it has been pointed out that there is a possible impediment to the gradual evolution of mimetic phenotypes from cryptic ancestors (Charlesworth \& Charlesworth 1975). In those prey gaining substantial protection from crypsis, any small step towards a mimetic phenotype would concurrently increase conspicuousness and fail to produce sufficient resemblance to the model to confer protection (Charlesworth \& Charlesworth 1975). Given that these early forms are edible, relatively conspicuous, and are easily distinguished from the model, such intermediate phenotypes should then be selected against. What then enabled the existing Batesian mimics to overcome this phenotypic gap? One explanation involves a two-stage process 
where the first step is a major gene mutation that modifies the phenotype so drastically that the species is mistaken for the model in a large proportion of predator encounters (Nicholson 1927; Sheppard 1962, 1967; Charlesworth \& Charlesworth 1975). This is followed by second stage where subsequent mutations gradually increase fidelity to the model. Another explanation, illustrated more recently, is that high model abundance combined with high model noxiousness can create conditions where even relatively poor mimics do equally well as cryptic forms (Kikuchi \& Pfennig 2009). Both of these mechanisms may have played a role in the widespread evolution of eyespots in caterpillars. A third route has been gaining acceptance more recently, which operates on the idea that predators pay close attention to certain features and ignore other features that could provide useful information. This process is sometimes called "overshadowing" and provides apparently "poor" mimics with some degree of security as long as they possess a feature that predators use to categorize prey as unsuitable (Gamberale-Stille et al. 2012; Aronsson \& Gamberale-Stille 2012).

\subsection{A brief introduction to eyespots}

Much of this thesis focuses on the evolution and anti-predator function of conspicuous circular markings, commonly called 'eyespots'. The term 'eyespot' comes from a widely held inference that these paired markings deceive predators by resembling eyes. Indeed eyespots might serve a variety of anti-predator functions which may or may not be attributed to eye mimicry (Stevens 2005, Table 1-1), and the last 10 years has seen renewed interest in these markings. As of 2010 when I began this work, research in this area had focused largely on fish and adult Lepidoptera (fish: Karplus \& Algom, 1981; adult Lepidoptera: Vallin et al. 2005; Olofsson et al. 2010), but were known to occur in other groups including Coleoptera (e.g., 
eastern-eyed click beetle, Alaus oculatus), Hemiptera (e.g., lantern fly, Fulgora lanternaria), Orthoptera (e.g., peacock katydid, Pterochroza ocellata), Mantodea (e.g., peacock mantis, Pseudempusa pinnapavonis; spiny flour mantis, Pseudocreobotra wahlbergi), and in the larvae of Lepidoptera (Janzen et al. 2010). Considering only those cases where eyespots operate defensively, the fact that eyespots have arisen independently (and been subsequently maintained) in so many distinct groups suggests two things: 1) the protective value of eyespots may be considerable, and 2) the means through which protection is conferred may be quite general. There are several mechanisms through which eyespots may confer protection to the animals which possess them, and these mechanisms are discussed in detail elsewhere (e.g., Edmunds, 1974; Kodandaramaiah, 2011; Stevens, 2005). Consequently, I outline these mechanisms here only briefly and instead spend more time in the next section discussing eyespots as they relate specifically to caterpillars.

The means through which eyespots are thought to protect animals from their predators can broadly be separated into deflection and intimidation. A number of studies in adult Lepidoptera have now convincingly demonstrated that small marginal eyespots can deflect a bird's strike away from their body, minimizing damage and facilitating escape (e.g., Lyytinen et al. 2004; Olofsson et al. 2010; Vallin et al. 2011). Recent evidence suggests that eyespots may serve such a deflection function in aquatic environments as well (Kjernsmo \& Merilaita 2013). It was suggested by Blest (1957) that the eyespots of many Papilio caterpillars may be "directive", in order to lure attacks towards the location of the osmeterium which releases noxious chemicals when caterpillars are harassed (Frankfater et al. 2009). Blest did find evidence that adding spots to mealworms could lure strikes (Blest 1957), but more recent studies have shown that the osmeterium seems to be ineffective in deterring bird attacks (Leslie \& Berenbaum 1990; 
Frankfater et al. 2009). Such directive effects might also explain the eyespots in some adult amphibians (e.g. Physalaemus nattereri) which are associated with glands that secrete highly noxious fluid (Lenzi-Mattos et al. 2005).

Instead of directing or deflecting attack, large eyespots are thought to intimidate attackers (Stevens 2005; Kodandaramaiah 2011; Kodandaramaiah et al. 2013), and a considerable body of evidence exists to support this claim (Vallin et al. 2005, 2006; Kodandaramaiah et al. 2009; Merilaita et al. 2011; Olofsson et al. 2012). However, research led by Martin Stevens has challenged the traditional assumption that these large eyespots are intimidating because they resemble the eyes of the predator's own enemies (Stevens 2005; Stevens \& Ruxton 2014). Stevens correctly points out the fact that large eyespots preventing or delaying a predator's attack does not in itself indicate that eyespots mimic eyes ("eye mimicry hypothesis") and could instead result from predators avoiding prey with highly conspicuous and/or novel signals (the “conspicuous signal hypothesis"; Stevens 2005, Kodandaramaiah 2011; Stevens and Ruxton 2014).

As the name implies, eyespots have traditionally been considered as features that mimic eyes. In some cases, this apparent eye mimicry is coupled with body parts in a way consistent with other head- or face-like features, further suggesting a mimicry function. In particular, many caterpillars combine eyespots with swollen body segments or behaviors leading many eminent naturalists and evolutionary biologists to consider them snake mimics (see below). Of course, the opinions of individual scientists, regardless of their eminence, is less valuable than rigorous empirical support. The next section reviews the historical context and summarizes the natural history and experimental work that had been done to inform our understanding of eyespots in caterpillars as of 2010. This cut off, though seemingly arbitrary, was used deliberately to reflect 
the state of our knowledge of this phenomenon when I planned and conducted the research detailed in the research chapters that follow.

\subsection{Eyespots in lepidopteran caterpillars}

Naturalists and evolutionary biologists alike have long recognized that a variety of lepidopteran caterpillars, to a greater or lesser extent, resemble snakes (e.g., Bates 1862; Weismann 1882; Wallace 1889; Poulton 1890; Moss 1912). This perceived resemblance is generated by a number of traits, differing among caterpillars, and acting either independently or synergistically to convey the impression of a snake. For instance, when Henry Walter Bates described his encounter with one such caterpillar (see Preface) he emphasized three major components which seem to characterize the majority of the so-called 'snake mimic' caterpillars: i) a pair of pupiliated eyespots located towards one end of the caterpillar (usually the anterior end, but occasionally the posterior end), ii) body segments that are enlarged or dilatable, and iii) bending behaviours expressed upon approach or harassment. As naturalists continue to discover additional lepidopteran species, or describe previously unobserved larval stages of a known species, it has become apparent that eyespots are by no means uncommon in caterpillars (Janzen et al. 2010; Janzen \& Hallwachs 2014). Indeed, eyespots seem to have arisen in numerous lineages within Lepidoptera, with each lineage expressing great diversity in shape, colour, and complexity of the eyespots themselves. This is illustrated well in a recent manuscript (Janzen et al. 2010), as well as in the photographic database provided by Janzen's website (Janzen \& 
Hallwachs 2014) and my Flickr group "Caterpillars with Eyespots" (https://www.flickr.com/groups/eyespots/).

Anecdotally, some of the most impressive examples of mimicry by lepidopteran larvae come from relatively large species, including many from the Sphingidae family. In fact the example that Henry Walter Bates recounted as being "extraordinary" was likely a sphingid species (see Preface) that can attain a sizable (for a caterpillar) $8.6 \mathrm{~cm}$ in length as a caterpillar, and other even larger examples exist (see Chapter 6). The description of this caterpillar by Bates is understandably brief as these caterpillars were not the immediate focus of his manuscript, but a more detailed description of the deceptive appearance and behaviour of this caterpillar is provided later by Moss (1920). This description can be read in full with accompanying photos in Appendix A, along with his description of a related sphingid, Madoryx pluto (now M. plutonis), which appears to have evolved a distinct form of snake mimicry seemingly independent from Hemeroplanes. Alfred Russel Wallace, a former collaborator of Bates, would note in his own book on natural selection that Sphingid caterpillars with eyespots terrify their enemies by resembling snakes (Wallace 1889, p209-210). Using similar language, E. B. Poulton (1890) would describe this in more detail a year later citing two European sphingids Deilephila elepenor and D. porcellus as representing impressive snake mimics that terrify their enemies (Poulton 1890).

Yet by far the most thorough early account of eyespot markings in Lepidoptera, through each of the instars, was given by the eminent evolutionary biologist August Weismann who discussed the appearance and function of eyespots (Weismann 1882). His main interest in eyespots and other larval markings was that tracking the changes in these markings through ontogeny could provide important insight into the relatedness among species. Importantly, 
Weismann made a clear, and biologically reasonable, distinction between two sets of superficially similar markings, which he called "eye-spots" and "ring-spots" (Figure 1-1). "Eyespots", as defined by Darwin and so considered also by Weismann, are "a spot within a ring of another colour, like the pupil within the iris, but the central spot is often surrounded by additional concentric zones" (Darwin 1882, pp. 427-428). For each component of the eyespots Weismann had corresponding terms, the central spot (pupil) he termed "nucleus", the lighter area surrounding the pupil was the "mirror", and the typically darker area surrounding this was the "ground-area". He proposed that eyespots were deceptive imitations of an eye and even conducted some early experiments with Deilephila porcellus and D. elpenor (species considered by Weismann to have "eye-spots", Figure 1-1AB) to demonstrate their protective effect against avian enemies. The so-called "ring-spots" differed from this in that they lack a nucleus, or pupil, indicating to Weismann that these markings were not imitating an eye. His preliminary experiments with feeding Hyles gallii (Figure 1-1C) to lizards indicated that these "ring-spot" markings may have an aposematic function, though he regretted not being able to properly conduct these experiments with birds and admitted that more rigorous work was needed. $\mathrm{He}$ suspected that $H$. euphorbiae (Figure 1-1D), H. dahlii, and H. mauritanica similarly used their conspicuous ring-spots as signals of distastefulness, because they all feed by day on poisonous Euphorbiaceae plants while fully exposed. Ring-spots in some other species, such as $H$. hippophaes, were thought to aid in concealment by resembling the berries of their food plant, but again he was prevented from testing this experimentally by logistical constraints. 


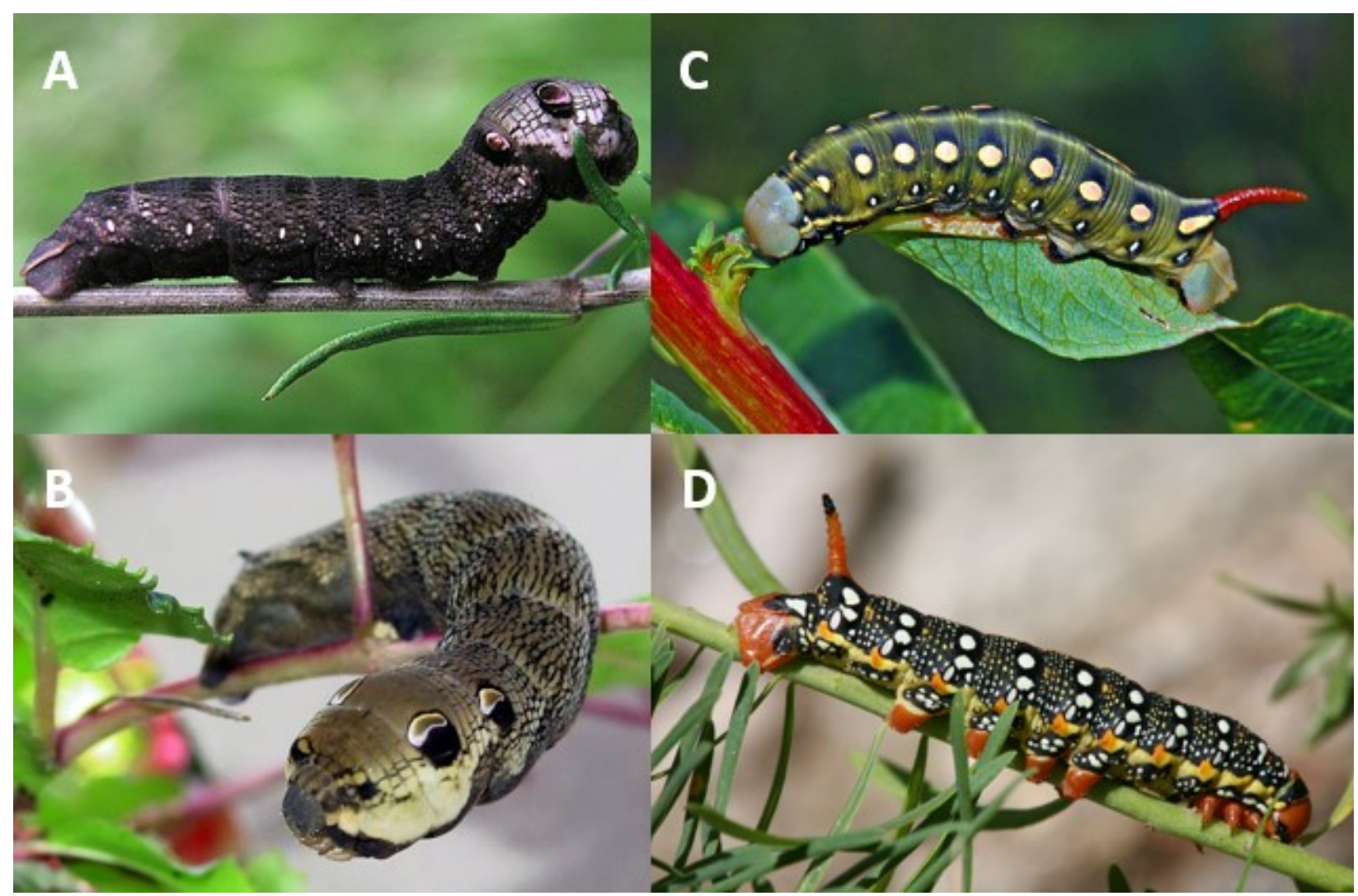

Figure 1-1: Final instar Sphingidae caterpillars illustrating what August Weismann's distinction between "eye-spots" (panels A, B) and "ring-spots" (panels C, D), which he considered to have distinct functions. A) Deilephila porcellus, photo by "NaturKamera"; B) D. elpenor, photo by Joachim Baecker; C) Hyles gallii, photo by "Hectonichus". H. gallii caterpillars also have a black colour variant; D) Hyles euphobiae, photo by Daniel Schwen. All four photos accessed from Wikipedia Commons and used freely as per their Creative Commons licence. 
An informative, if humorous, anecdote about the frightening appearance of some sphingid caterpillars comes from the footnotes in another tome on the larvae of British butterflies and moths (Buckler 1887, p. 115) and states:

"The general aspect of this larva [D. elpenor], with its puffed front segments, and the curious blotches on segments 5 and 6 makes it a thing of horror to the non-entomological finder. Mr. Bignell tells me of a whole brood, which were discovered feeding on a vine in a back garden, and bravely captured with the kitchen fire tongs, and put to death as venomous beasts."

Anecdotes like this and the one from Bates included in the preface are explicit examples of how effectively the traits of these caterpillars seem to be in their deception, reflecting the widely held belief that the eye-like markings on caterpillars do in fact mimic the eyes of a dangerous vertebrate. Of course these eye-like markings, and the associated perception that they resemble the eyes of a snake, is not limited to Sphingidae. An early detailed account of the snake-like appearance of Papilio palamades and P. troilus (Papilionidae) caterpillars appears in Copeia, a herpetology and ichthyology journal (Pickens 1927). In addition to describing the apparent "head" shape and eyespots as consistent with that of a snake, Pickens seems to have been the first to highlight the osmeterium as potentially augmenting the mimicry by resembling a snake's tongue (Pickens 1927). Pickens correctly states that this forked structure, which extends from behind the caterpillar's true head, happens to be correctly coloured and located (in relation to the eyespots and other head-like features) where one would expect to observe the snake's tongue (see Figure 1-2). Today many Papilio caterpillars are still widely considered snake mimics, despite the fact that they attain maximum sizes of around $6 \mathrm{~cm}$ in length (e.g., see the description of Papilio troilus in Wagner, 2005). Certainly many caterpillars resemble snakes at least to the 
human observer, and among naturalists and evolutionary biologists alike it seems to have become generally accepted that caterpillar eyespots increase a caterpillar's resemblance to a snake, thereby functioning to scare off predators such as insect-eating birds. 


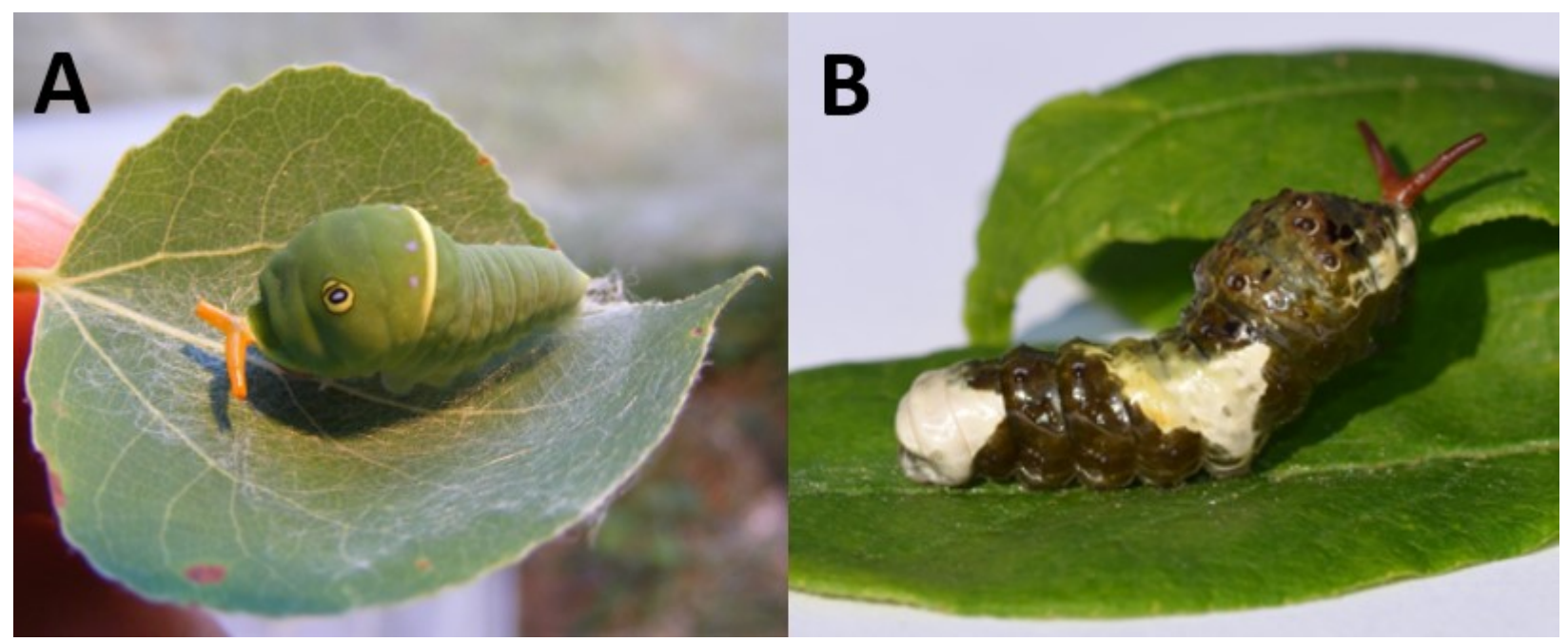

Figure 1-2 Final instar Papilio genus (Papilionidae) caterpillars in their defensive posture, everting their osmererium from behind their head. Though this structure likely functions to deter insect or parasitoids, and emits volatile chemicals. Many have argued that the osmeterium has been co-opted to facilitate snake mimicry in at least some Papilio species. This is because the structure resembles a snake's tongue because of its orange-red colour, location in relation to other eye- and face-like features, and the fact that it is forked. A) Papilio canadensis; B) Papilio cresphontes, a bird-dropping mimic which appears to retain comparable defensive posture and vestigial eyespots. Both photos by T. Hossie in Kemptville, ON, summer of 2011, and 2013 respectively. 
Yet, the empirical support for such claims is limited. Empirical support amounts to poorly replicated and scantily described experiments from Weismann and Poulton, and a few others, involving few ecologically relevant predators. One interesting, set of experiments was conducted by Shirota (1980). Briefly, Shirota affixed a pair of orange paper disks (ranging in size from $0.25-2 \mathrm{~cm}$ in diameter, the larger eyespot treatments being bigger than the diameter of the caterpillars themselves!) each with a concentric black spot to the anterior end of silkworm (Bombyx mori) caterpillars, then caterpillars were exposed to field caught starlings (Sturnus cineraceus) in the lab. These experiments showed that birds delayed attacking caterpillars with larger eyespots, directed strikes towards small eyespots, and preferred no-eyespot caterpillars over caterpillars with eyespots only when spots were $1 \mathrm{~cm}$ or greater in diameter (Shirota 1980). Finally, late instar larvae of D. elpenor lewisii were exposed to the starlings. These caterpillars were never attacked and birds were reportedly "restless" and "frightened" by the larvae despite the caterpillar's eyespots being only $0.4 \mathrm{~cm}$ in diameter (Shirota 1980). From this, we can conclude that although the protection afforded by 'eyespots' may increase with larger spots (see also Stevens et al. 2008a), the effects of additional traits yet to be quantified (e.g. eyespot position, morphology, behaviour) are likely critical to effective predator intimidation.

In the 33 years following the work by Shirota (1980), to my knowledge, there has been no experimental work examining eyespots in caterpillars. Yet, it is clear from the results of Shirota (1980), and from the dearth of other rigorous experimental work, that currently our mechanistic understanding of caterpillar eyespots is poor. That being said, it seems likely that the shape of the apparent "head" and the suite of defensive behaviours are likely to play a role as 
these features seem to be shared among many caterpillars with eyespots, and could conceivably make the caterpillars look more snake-like.

A thoughtful review of snake mimicry in caterpillars was included as a primary example in F. Harvey Pough's 1988 manuscript “Mimicry of vertebrates: are the rules different?” (Pough 1988). Unlike other biologists who have been reluctant or guarded in ascribing snake mimicry specifically as the function of eyespots and other related traits (e.g., Pickens, 1927), Pough clearly views this system in the light of Batesian mimicry. Importantly he points out that for these caterpillars that resemble snakes there is no one-to-one model-mimic relationship, a pitfall into which many non-specialists fall. This is followed up with several solid arguments for why the mimicry of snakes tends to be comparably abstract. The most convincing, and perhaps general, argument is that snakes are have properties as models that differ from most groups. Both venomous and non-venomous snakes can be extremely noxious in that the risk of rapid death for the agent of selection (i.e. insect-eating birds) is high in both cases. Pough argues that even the consequences of sub-lethal interactions experienced upon surviving an interaction with a snake, such as tissue necrosis and infection, can be so severe as to render them virtually as critical as immediate death. Thus, snakes are extremely noxious models, which causes predators to broadly generalize their aversion to snakes, resulting in even marginal resemblances to a snake conferring substantial protection to a caterpillar (Pough 1988). In addition, unlike most mimicry systems, predators cannot sample their model population to assess noxiousness or learn how to discriminate models and mimics when the model is lethal. Even in cases where the predator succeeds in killing a model, it would not learn to avoid them in subsequent interactions. This "paradox of a deadly model" is solved by selection for innate avoidance, and such innate fear of snakes has been widely reported (Reviewed in Pough 1988). Taken together this innate fear and 
broad generalization resulting from extreme noxiousness of snakes as models explains why abstract mimicry of snakes by caterpillars is maintained and is so widespread (Pough 1988).

Just as I finished writing my proposal to work on the subject of caterpillar eyespots, a Perspective piece was published in the journal Proceedings of the National Academy of Sciences by some of the world's experts in lepidopteran larvae and tropical ecology - Daniel Janzen, Winnie Hallwachs, and John Burns (Janzen et al. 2010). This paper was entitled "A tropical horde of counterfeit predator eyes", and nicely laid out a modern framework for understanding eyespots as they occur in caterpillars. In many ways this was an update and extension of Pough's treatment of the subject (Pough 1988), but focused more explicitly on caterpillar eyespots. Specifically, the paper outlines numerous relevant aspects of tropical bird and caterpillar natural history that has not previously been outlined explicitly. Of course, this context is critical if one hopes to attempt an understanding of such complex tropical ecosystems (Janzen 1988). The paper also provides a beautiful gallery of larvae and pupae to illustrate how widespread and diverse the phenomenon seems to be.

The central argument of Janzen et al (2010) is that the plethora of tropical caterpillars with eyespots is the product of a multispecific (i.e. multiple species) set of avian predators that possess an innate fear of eye or face-like stimuli (Janzen et al. 2010). The authors are cautious in attributing all the innate avoidance of eye- or face-like features to fear of snakes, stating instead that "These false eyes are mimicking the eyes and faces of such predators of insect-eating birds as snakes, lizards, other birds, and small mammals, as perceived at close range by the insectivorous birds in their natural world" (Janzen et al. 2010). As with Pough (1988), Janzen and colleagues stress the importance of a predator's innate aversion to features that indicate danger or noxiousness during the evolution of prey phenotypes. In cases where innate reactions 
are the predator's dominant response, fundamental aspects of the system will differ from those systems understood in terms of experience, memory and learning potential (Janzen et al. 2010). Specifically, when fear is innate it is no longer difficult to explain why in these systems mimics frequently outnumber their models, lack specific models, frequently appear to have poor levels of mimetic fidelity, and often occur in allopatry from their models (see also Pfennig \& Mullen 2010). Indeed, the suggestion that innate fear is driving the evolution and maintenance of this multi-specific mimicry complex is synthetic and elegant in how it enables us to understand this system. Of course, this leaves the question under what circumstances should innate fear evolve and be maintained?, an area of research surprisingly deficient in theoretical and empirical work (but see Plough 1988). Innate fear seems likely to evolve in cases where learning is prevented by the high costs associated with attacking a deadly model (Pough 1988).

Perhaps surprisingly, as of 2010 when I began this work there was a clear deficiency of empirical support for many of the fundamental aspects of the argument that eyespots protect caterpillars from insect-eating birds as a result of an innate fear of snakes and/or other threatening vertebrates. Robust empirical tests had not been conducted to examine the key questions do eyespots confer protection to caterpillars? and, do caterpillars with eyespots resemble snakes (or other predatory vertebrates) to non-human observers (i.e., to the true agents of selection)? This thesis attempts to address these and related questions by conducting rigorous empirical work. I reproduce here a supplementary table from my first publication on this topic (Hossie \& Sherratt 2012) which I think usefully summarizes all the possible functions of caterpillar eyespots and provides the references for the work on those functions conducted prior to 2012 . 


\section{Table 1-1 Outline of possible nonmutually exclusive functions of eyespots in lepidopteran caterpillars and the relevant literature}

\begin{tabular}{ll}
\hline Possible function & Comments \\
\hline $\begin{array}{l}\text { Eye mimicry: eyespots protect } \\
\text { prey because they resemble the } \\
\text { eyes of dangerous predators (e.g. } \\
\text { snakes) }\end{array}$ & $\begin{array}{l}\text { Classic explanation and prevailing } \\
\text { hypothesized function for eyespots } \\
\text { on caterpillars, especially within the } \\
\text { naturalist community }\end{array}$ \\
$\begin{array}{l}\text { Startle signal: eyespots are } \\
\text { conspicuous markings that startle } \\
\text { attackers }\end{array}$ & $\begin{array}{l}\text { Markings scare off attackers because } \\
\text { they are conspicuous and presented } \\
\text { suddenly }\end{array}$ \\
\hline
\end{tabular}

\begin{tabular}{ll} 
Empirical work related to caterpillars & Additional references \\
\hline $\begin{array}{l}\text { None, although Janzen et al. (2010) } \\
\text { provides natural history observations } \\
\text { and theoretical grounds to support this }\end{array}$ & Caterpillar eyespots: Bates 1862; \\
Poulton 1890; Edmunds 1974; \\
Janzen et al. 2010
\end{tabular}

mechanism

Adult Lepidoptera: Stevens 2005; Kodandaramaiah 2011; Merilaita et al. 2011

conspicuous markings that startle suddenly

None

With moth-like prey: Blest 1957;

Cundy \& Allen 1988

Reviewed in reference to eyespots: Stevens 2005; Kodandaramaiah 2011

General review: Ruxton et al. 2004 (Section 13.5)

Conspicuous signal: predators avoid attacking prey that possess conspicuous markings

Direction: eyespots direct attacks towards additional defences
Includes effects of 'dietary

conservatism' and 'neophobia'. It

seems possible that birds avoid prey with eyespots simply because

eyespots are unfamiliar

Seems plausible for several species (e.g. Papilio spp., Cerura vinula). Blest (1957) suggested it specifically for papilionid larvae with osmeterium
Coppinger 1970; Schuler \& Hesse 1985; Roper \& Cook 1989; Roper 1990; Stevens 2005; Stevens et al. 2007, 2008a

Blest (1957) analysed where captive birds pecked mealworms painted with a single spot
Poulton (1890) details the spraying of irritant fluid from anterior end (near eyespots) of C. vinula larvae when touched 


Deflection: eyespots deflect
attacks away from vulnerable body
parts (e.g. head capsule)

Warning signal: eyespots act as a warning signal indicating unpalatability

Distraction: eyespots increase crypsis by distracting attention from the true edge of prey
Refuted by Janzen et al. (2010) on

the grounds that attack to any part of

a caterpillar's body would be lethal.

However, reducing the probability of

strikes to caterpillar's true head could

have some benefit

Unlikely. Caterpillars with eyespots tend to be palatable. In the Papilio genus, aposematism is not linked to the presence of plant toxins (Prudic et al. 2007)

Small conspicuous markings reduce detection of prey by distracting

predators' 'attention' away from the true outline of a prey item

\section{None}

Evidence that eyespot caterpillars are palatable: Brower 1958; Leslie \&

Berenbaum 1990; Takagi et al. 1995

None with caterpillars

Suggested by Thayer 1909

Tested with moth-like targets by Stevens et al. 2008b (not supported) 
More generally, I hope that this work will provide insight into the evolution of anti-predator defences. A number of factors make lepidopteran caterpillars a useful system for this. Firstly, caterpillars are a pre-reproductive stage in development which is followed by complete metamorphosis (holometabolism) to a distinct adult form. As a result, the function of a phenotype expressed in the larval stage is therefore less obfuscated by sexual selection than comparable adult phenotypes. In addition, Lepidoptera is a diverse and well-studied group which includes several closely related species that employ a diverse range of anti-predator defences including crypsis, aposematism, and spinescence (Lederhouse 1990). The extent of available gene sequences and robust phylogenies also facilitates any comparative work conducted in this system by enabling researchers to control the extent of non-independence among specieslevel data. Over the course of my PhD I have utilized this valuable system to examine the ecology and evolution of anti-predator defence by specifically investigating eyespots in caterpillars.

\subsection{Thesis Overview}

I designed my doctoral research program to follow the logical steps required to develop a comprehensive and empirically supported understanding of caterpillar eyespots. I began by conducting the first empirically sound experiment to test whether eyespots can confer protection to caterpillars exposed to wild predators (Chapter 2). Eyespots did confer protection, but this protection was dependent upon other aspects of body colour and was not as strong as one might expect given the widespread nature of the phenomenon. I therefore tested whether the caterpillar's defensive posture might increase the protection afforded by eyespots perhaps because they place these eye-like markings in 
the context of a "head" (Chapter 3). Despite the finding that the defensive posture indeed strongly reduced predation on the caterpillars, I was left without evidence for or against the contention that this protection resulted from an increase in mimetic fidelity to snakes. Thus, I set out to explicitly test whether a shift from a resting posture to the defensive posture really does result in a concurrent increase to mimetic fidelity to snakes (Chapter 4). I found that the defensive posture primarily increases resemblance to Viperidae snakes, and not snakes more generally, and only when viewed from above. Chapter 5 is a natural history description of a pair of caterpillars which possess the unique ability to palpitate their anal eyespot such that it creates (at least to human observers) the appearance of a blinking vertebrate eye. This is an important work because it illustrates that eye-mimicry in caterpillars is not restricted to specific mimicry of snake eyes (which cannot blink). Now, armed with an empirically-based foundation, I set out in Chapter 6 to explain the distribution of eyespots in caterpillars - specifically, why do some caterpillars have eyespots while others do not? This was accomplished using a combination of the phylogenetic comparative method, a field experiment with wild birds, and a lab experiment with naïve domestic chicks. This chapter illustrates that eyespots evolve in relatively large species as a result of selection against eyespots in small prey, and for eyespots in large prey. I end with a general conclusions chapter that summarizes my current thoughts on how the system operates, what I feel this work has accomplished more broadly, and where I see future research in this area being most valuable.

Based on the body of research I have conducted over the past four years my overarching thesis is that eyespots are an adaptation that has arisen in large caterpillars 
to protect them from a multispecific community of insect-eating birds which fear snakes and other dangerous vertebrates. 


\title{
2 Chapter: Eyespots interact with body colour to protect caterpillar- like prey from avian predators
}

\author{
Thomas J. Hossie ${ }^{1 *}$ and Thomas N. Sherratt ${ }^{1}$ \\ ${ }^{1}$ Department of Biology, Carleton University, Ottawa, ON, Canada \\ *Corresponding author
}

Published in Animal Behaviour (2012) 84: 167-173

Reproduced here in whole in accordance with Elsevier terms and conditions. 


\subsection{Abstract}

A wide range of animals possess conspicuous eye-like markings on their bodies, commonly called 'eyespots'. These markings are generally thought to serve an antipredator function, particularly in lepidopteran caterpillars where eyespots may mimic the eyes of their enemies' predators. Despite this, the protective value of caterpillar eyespots remains unclear and has yet to receive empirical study. Moreover, most eyespot caterpillars are otherwise cryptically coloured, suggesting that the efficacy of eyespots may depend on additional aspects of prey coloration. We assessed the protective value of caterpillar eyespots with and without a form of countershading by pinning artificial caterpillars to tree branches in the field and monitoring their survival over $90 \mathrm{~h}$. Eyespot and countershading treatments were arranged in a $2 \times 2$ factorial design, allowing us to quantify both main and interactive effects. We also photographed attacked prey to determine whether eyespots affected the area of the prey's body that was attacked (i.e. pecked by an avian predator). We observed no main effect of eyespots or countershading on survival; instead, countershading and eyespots acted synergistically to reduce predation, although whether this interactive effect reflects a countershading mechanism or different background matching when prey are viewed from above is unclear. Importantly, bird pecks were directed both towards eyespots and towards the body section furthest from the eyespots. This is the first field experiment to examine the protective value of eyespots on caterpillar-like prey and demonstrates that the extent of protection depends on additional factors, including body colour and possibly behaviour. 


\subsection{Introduction}

Many animals possess conspicuous circular markings that superficially resemble vertebrate eyes. These markings are commonly called 'eyespots' and while they may play a variety of roles (e.g. sexual signaling; San Martin et al. 2011), they are widely thought to have evolved as an antipredator defence (Blest 1957; Edmunds 1974; Stevens 2005; Kodandaramaiah 2011; see Table 1-1 for a summary of possible antipredator functions of eyespots). Accordingly, the possession of eyespots may deflect a predator's attack away from vulnerable body parts and/or prevent attacks altogether through predator intimidation. Nevertheless, empirical tests of these proposed mechanisms have been partly contradictory (intimidation: Vallin et al. 2005; Stevens et al. 2007; Merilaita et al. 2011: deflection: Lyytinen et al. 2003, 2004; Olofsson et al. 2010), and it remains unclear the extent to which eyespots increase prey survival under field conditions. Moreover, when such tests have been conducted they have sometimes challenged popular belief. For example, recent work has suggested that the intimidation effect of eyespots, at least in adult Lepidoptera, may be a consequence of predators avoiding prey with conspicuous markings, rather than eyespots resembling the eyes of a predator's own enemies (Stevens et al. 2008b; Stevens \& Merilaita 2009).

Regardless of the specific mechanism, the fact that eyespots have arisen independently in several animal groups (Ruxton et al. 2004a), and in a range of lifehistory stages, indicates that their protective value may be considerable. Eyespots occur on caterpillars from numerous independent lineages within Lepidoptera and have long been believed to confer protection against insectivorous birds through enhancing their resemblance to predatory snakes (e.g. Poulton 1890; Edmunds 1974; Chittka \& Osorio 
2007). Indeed, in his classic monograph, Henry Walter Bates described a caterpillar with eyespots that resembled a snake as 'The most extraordinary instance of imitation I have ever met' (Bates 1862, p509). Most recently, Janzen et al. (2010) detailed the natural history of many Neotropical caterpillars with eyespots and proposed that these markings represent an eye-mimicry complex generated by avian predators, which innately fear predator eyes. Yet while predator eye mimicry is entirely plausible, to date no experimental work has examined this function for caterpillar eyespots in the field. Specifically, we lack empirical evidence showing that (1) eyespots protect caterpillars from avian predators, and that (2) eyespot function in caterpillars is related to the mimicry of snake or other predators' eyes, as opposed to the more general effects of neophobia or dietary conservatism.

To date, only a few preliminary attempts have been made to understand the function of eyespots in caterpillars. For instance, Poulton (1890) described three simple trials (each using only a single caterpillar) that supported an intimidation function for caterpillar eyespots; however, this work was anecdotal, and the eye-spotted caterpillar in question was eventually consumed in two of the three cases described. Work by Blest (1957) found that captive birds directed their pecks towards a single eyespot that had been painted on mealworm larvae. Shirota (1980) likewise presented caterpillars with artificial eyespots to six captive birds, but found that all caterpillars were promptly attacked, with delays to attack only occurring when eyespots were unrealistically large (i.e. diameter of $1-2 \mathrm{~cm}$ ). Intriguingly, the same birds did not attack a live Deilephila elpenor lewisii caterpillar, which naturally possesses eyespots (Shirota 1980). Therefore, 
each of the above studies only provides anecdotal observations, and the protective value of eyespots within a natural setting remains to be demonstrated.

The caterpillars of many lepidopteran species are generally thought to mimic snakes as they possess a pair of eyespots on enlarged anterior body segments during late instars (e.g. Papilio sp., see Scriber et al. 1995; Allen et al. 2005; Wagner 2005 for photos). Several of these species are abundant in extratropical areas that lack the snake species that might pose a significant threat to birds. In these instances, eyespots could continue to be maintained by migratory birds that express either a learned or innate aversion to predatory snakes encountered in tropical wintering grounds, or a more general innate aversion to larger creatures with eyes (Janzen et al. 2010). As several species of caterpillars that have eyespots are apparently palatable, it seems unlikely that eyespots function as warning signals (Brower 1958; Leslie \& Berenbaum 1990; Takagi et al. 1995). A deflection function for caterpillar eyespots has also been dismissed previously on the grounds that damage to any part of a caterpillar's body would be lethal (Janzen et al. 2010); however, in some cases, eyespot-targeted strikes would direct attacks towards specialized organs that release noxious chemicals when caterpillars are harassed. For example, Blest (1957) suggested that eyespots of Papilio caterpillars might direct attacks towards the osmeterium (Frankfater et al. 2009). In addition, deflecting pecks away from the true head might allow caterpillars to mount behaviours that heighten the intimidation effect of eyespots, thereby thwarting attacks before lethal damage is done. Experimental work in the field is clearly needed to clarify whether caterpillar eyespots function to 
intimidate predators from attacking and/or whether the presence of eyespots also serves to deflect or direct strikes.

The efficacy of eyespots in defending against predators may be affected by additional features of the prey's coloration. For instance, in a field experiment using moth-like targets, Stevens et al. (2008) showed that eyespots reduced predation on conspicuous, but not camouflaged, prey. Yet, most eyespot caterpillars are cryptically coloured (Poulton 1890; Edmunds 1974; Janzen et al. 2010), and many 'snake mimic' caterpillars are countershaded (e.g. Papilio canadensis, Papilio glaucus: Wagner 2005). Other potential examples of caterpillars with eyespots that appear countershaded include Hebomoia glaucippe (Pieridae), Daphnis nerii (Sphingidae), Xylophanes ceratomioides (Sphingidae) and Hemeroplanes triptolemus (Sphingidae). As countershading (i.e. darker pigmentation on surfaces is generally illuminated) has been suggested to be an effective means of crypsis in caterpillars (Rowland et al. 2007, 2008), then it is possible that eyespots and countershading have an interactive effect on caterpillar survival.

To determine whether constantly displayed eyespots were effective in deterring avian predation and/or deflecting strikes, we deployed artificial caterpillars in the field and monitored their 'survival'. Specifically, caterpillar treatments followed a $2 \times 2$ factorial design: with or without 'eyespots' and with or without countershading. This design allowed us to compare directly the survival of prey with eyespots against two alternate prey with greater levels crypsis (i.e. solid green-no eyespots, and countershadedno eyespots). Furthermore, our design permitted us to test for an eyespot-countershading interaction. Overall, we predicted that eyespots would protect prey by intimidating 
predators from attacking, and if eyespots serve a deflection function, we predicted that pecks would be directed towards the caterpillar's eyespots.

\subsection{Methods}

\section{Site Description}

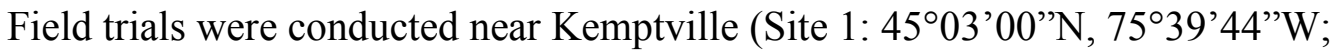

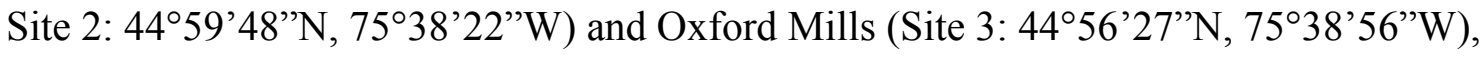
Ontario, Canada. Experiments were run between 25 May and 27 August 2010, which corresponds roughly to the dates when Canadian tiger swallowtail, Papilio canadensis, caterpillars could be found in the wild in our study area (Hall et al. 1996; T. J. Hossie, personal observation). All three sites were composed primarily of secondary growth of mixed deciduous forest. Each site was no closer than $5.5 \mathrm{~km}$ from another site. Preliminary scouting of each site confirmed the presence of $\mathrm{Pa}$. canadensis adults, snakes, as well as several species of insectivorous birds. Insectivorous bird species observed in each of the field locations are detailed in appendix B Table S2-1.

\section{Artificial Prey}

Artificial caterpillars were designed to assess the protective value of eyespots and countershading on caterpillar prey in general; however, for ecological relevance, our prey were modelled loosely on Papilio canadensis. We created artificial prey following similar protocols to previous studies (e.g. Church et al. 1997; Rowland et al. 2008). Specifically, pastry caterpillars were manufactured with a 3:1 mixture of white flour (White Rose ${ }^{\mathrm{TM}}$, White Rose Flour Mills, Byford, WA, U.S.A.) and lard (Tenderflake ${ }^{\mathrm{TM}}$, Maple Leaf Foods, Toronto, ON, Canada). From this, we made light green pastry by 
adding three drops of Leaf Green AmeriColor Soft Gel Paste ${ }^{\mathrm{TM}}$ (AmeriColor Corp., Placentia, CA, U.S.A.) and $20 \mathrm{ml}$ of water, and we made dark green pastry by adding six drops of gel paste and $5 \mathrm{ml}$ of water. Strips of dark and light green pastry were produced by pressing the dough through a circular opening $0.5 \mathrm{~cm}$ in diameter using a modified Play-Doh ${ }^{\mathrm{TM}}$ Mini Fun Factory ${ }^{\mathrm{TM}}$ (Hasbro Canada Corp., Longueuil, QC, Canada); these strips were then cut into $4 \mathrm{~cm}$ lengths. To make uniform, light green prey, two $4 \mathrm{~cm}$ strips of light pastry were pressed together and rolled such that the final artificial prey were cylinders $4 \mathrm{~cm}$ long with a diameter of about $7 \mathrm{~mm}$. Countershaded prey were produced using the same methods, but combining one light and one dark strip of pastry. This created a two-toned prey similar to Rowland et al. (2008); for our purposes the dark side was considered dorsal, as prey were presented on the upper side of branches. We used Elmer's ${ }^{\mathrm{TM}}$ Non-toxic Tempera Paint (Elmer's Products, Inc., Westerville, OH, U.S.A.) to create eyespot treatments, by painting two $0.6 \mathrm{~cm}$ black circles dorsolaterally on the prey, $0.3 \mathrm{~cm}$ apart and $0.5 \mathrm{~cm}$ from one end. Excess black paint was removed from the centre of the black spots using a thin paintbrush and a $0.4 \mathrm{~cm}$ white circle was painted inside each. Relatively large eyespots were used in an attempt to maximize the antipredator effect of the markings, as larger eyespot size greatly influences avoidance behaviour of predators (Stevens et al. 2008b). A dorsal view of an eyespot caterpillar is provided in appendix B Fig. S2-1. Tempera paint is an appropriate medium for eyespots as it dries in a smooth matte finish and the colours do not change over time (Mayer 1985). Finished pastry caterpillars (e.g. Fig. 2-1) were checked against a standard model prey for each treatment to ensure uniformity in size and shape of the body, as well as the size and position of eyespots. In this experiment we did not attempt to mimic the thickened head 
area that many eyespot caterpillars (e.g. Pa. canadensis) exhibit in their defensive posture. Although of interest, this thickening is expressed facultatively upon harassment, and here we focused on continuously displayed signals. Similarly, the caterpillars of a number of species (e.g. Deilephila elpenor, Xylophanes tersa) possess several pairs of eyespots. Whether additional pairs of eyespots serve a specific function or affect caterpillar survival remains untested; however, here we focused only on a single pair of eyespots. 


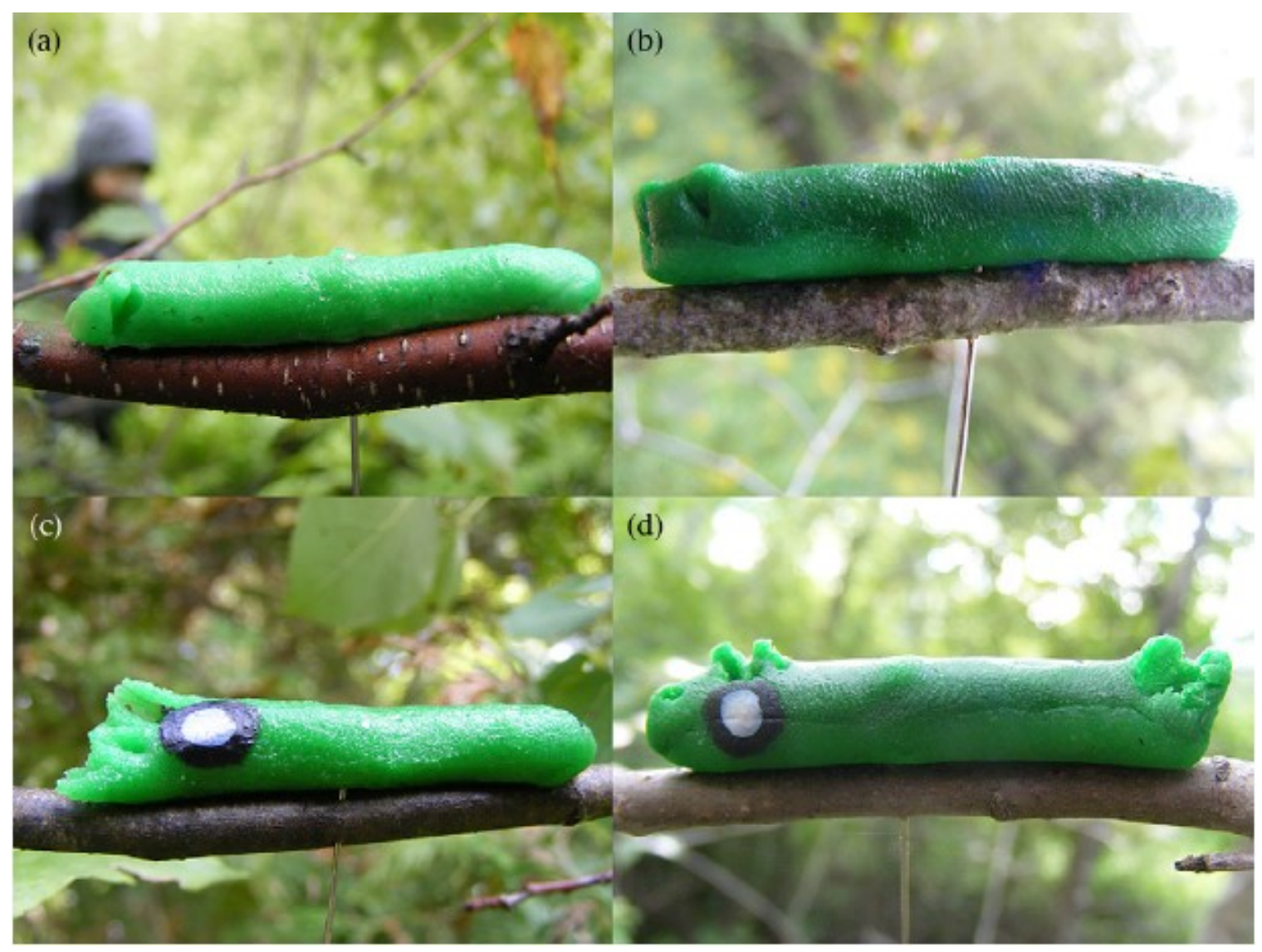

Figure 2-1: Examples of pecked pastry caterpillars from each of the four treatments used in this experiment. (a) Solid-no eyespots; (b) Countershaded-no eyespots; (c) Solid-eyespots;

(d) Countershaded-eyespots. 


\section{Prey Presentation}

Pastry caterpillars were deployed between 1700 and 1900 hours on the first day of a replicate site. Specifically, 24 trees were selected along a linear transect ( $\sim 10 \mathrm{~m}$ apart), and to each tree a single artificial prey was pinned on the upper side of a live tree branch in the north, south, east and west zone of a tree. Trees were restricted to one of four species (Populus tremuloides, Populus deltoides, Betula papyrifera, Acer rubrum; appendix B Table S2-2), which have all been identified as a host species of 'snake mimic' Papilio caterpillars (Scott 1986; Scriber et al. 1995; Allen et al. 2005). Each tree received a single pastry caterpillar from each treatment, and branch zone (i.e. north, south, east and west) was allocated randomly. Attaching four prey to each tree has the benefit of reduced variation in microhabitat among treatments. Selected branches were 1$2 \mathrm{~m}$ from the ground and were roughly $5-9 \mathrm{~mm}$ in diameter. Pastry caterpillars were considered killed if they were no longer present or had been attacked by a bird (peck marks present; e.g. Fig. 2-1). 'Survival' was checked at 14, 19, 24, 38, 43, 48, 62, 90 h from deployment. In all cases of missing baits, the pin (invariably still stuck in the tree branch) was found and the ground underneath was searched for the bait or any remaining pieces of an attacked bait. All attacked prey were photographed, then removed. Artificial prey damaged by rain, slugs or ants were removed $(N=88)$ and considered censored (i.e. considered to have survived only to that time period, but not considered killed) in the final analysis. After $90 \mathrm{~h}$, remaining prey were removed.

The entire process was replicated nine times (three times per site, albeit at a different location within site) for a total of 864 artificial prey deployed. Each consecutive replicate was conducted in an entirely new location within one of the three larger sites, 
and was at least $200 \mathrm{~m}$ from any previous location within that site. To avoid temporal confounds among sites, we always conducted consecutive replicates at a different site in a systematic order (i.e. Site 1, location 1; Site 2, location 1; Site 3, location 1; Site 1, location 2 , etc.). We conducted additional checks for pecked pastry caterpillars in the early replicates, but time constraints precluded us from checking for pecked pastry caterpillars in the remaining replicates. Specifically, in one replicate trial, a check for pecked/missing pastry caterpillars was conducted at $67 \mathrm{~h}$ from the start of the trial, and in two replicate trials, a $72 \mathrm{~h}$ check was conducted. Pastry caterpillars were designed to be fully palatable to birds, and our experimental design was approved by the Carleton University Research Ethics Committee (Protocol B10-35).

Analyses

We analysed the survival of artificial prey using Cox proportional hazards regression (Cox 1972), which can handle both censored data and nonuniform changes in predation risk. Since pastry caterpillars were pinned singly on branches, individual caterpillars may well have been encountered independently on the trees (e.g. Rowland et al. 2008); however, we controlled the possible lack of independence between baits on the same tree by clustering the baits by tree in our analysis. Overall significance of the model was assessed using the Wald test, which does not assume independence of observations within a cluster. Factors in our model included colour (i.e. solid versus countershaded), eyespot (i.e. eyespots versus no eyespots), and the colour×eyespot interaction. The effects of location and tree species were not of direct interest, and we did not have a priori hypotheses regarding their effect on survival. However, preliminary analysis indicated 
that the hazard rate differed among tree species and locations (see also appendix B Figs S2-2, S2-3). We therefore stratified the analysis to permit baseline hazard rates specific to location and to tree species. This analysis was conducted in R (R Development Core Team 2014) using the survival library (Therneau 2014), and the assumption of proportionality was tested using the cox. zph function.

We quantified the number and location of bird pecks on pastry caterpillars by analysing digital photographs of all retrieved baits that showed evidence of being attacked. For each pastry caterpillar with eyespots (i.e. with or without countershading), we counted the number of pecks in each of six evenly sized, continuous sections: anterior tip, body section with eyespots, mid-anterior body, mid-posterior body, hind-mid posterior body, and posterior tip. Similarly, for caterpillars without eyespots, we counted the number of pecks in each body section, with the anterior-posterior designation assigned randomly by a coin toss. To determine whether the number of pecks differed between body sections or treatments, we used a generalized mixed effects model with a Poisson error distribution and logit link function. We included section as a fixed factor and set individual as a random factor to control for the lack of independence of multiple pecks on a given caterpillar. The significance of a factor was examined by comparing models with and without the parameter of interest using a log likelihood ratio test. We also compared the fit of our models using Akaike's Information Criterion (AIC). This 
analysis was conducted in R (R Development Core Team 2014) using the lmer 4 package (Bates et al. 2011).

\subsection{Results}

The total $90 \mathrm{~h}$ mortality rate for a location ranged from $32 \%$ to $91 \%$ (mean \pm SE: $68 \pm 6.32 \%)$. The main effects of colour and of eyespots did not explain a significant amount of the variation in survival (Wald $=0.73, P=0.47$; Wald $=1.58, P=0.11$, respectively), but a significant eyespot $\times$ colour interaction was detected (Wald $=-2.36, P$ $=0.019)$. To interpret the significant interaction, we conducted pairwise Cox proportional hazard regressions on experimental treatments. We found a strong reduction in the hazard of countershaded prey with eyespots relative to solid prey with eyespots $(\sim 15.4 \%$ reduction; Wald $=5.05, d f=1, P=0.025$ ). Remaining pairwise comparisons were not significant (results from all pairwise Cox proportional hazard models are presented in Fig. 2-2a). Therefore, the significant interaction indicated that eyespots increased hazard on solid prey but decreased hazard on countershaded prey. Note that qualitatively equivalent results were obtained when missing prey were censored instead of classified as prey killed (appendix B Table S2-3). 
Table 2-1: Results from fitting Cox proportional hazards regression model of pastry caterpillar survival stratified by location and tree species

\begin{tabular}{lllllll}
\hline & Coef & Exp(coef) & SE(coef) & Robust SE & Wald & $P$ \\
\hline Colour & 0.04576 & 1.04682 & 0.11343 & 0.06303 & 0.726 & 0.4678 \\
Eyespot & 0.10715 & 1.1131 & 0.11316 & 0.06766 & 1.583 & 0.1133 \\
Colour×eyespot & -0.22295 & 0.80016 & 0.16243 & 0.09466 & -2.355 & 0.0185
\end{tabular}

Parameter estimates and associated significance tests depict how eyespots and countershading affect survival of pastry caterpillars. Since fates of pastry caterpillars that were pinned to the same tree were not necessarily entirely independent, we clustered caterpillars that were pinned to the same tree in the analysis and used the robust standard error to calculate the Wald statistic. 
Given that the baseline hazard rate differed between tree species (appendix B Fig. S2-3), we compared treatment-related survival by conducting further Cox regression models separately for each tree species. With the exception of Populus tremuloides, there was no significant difference in the survival rate of caterpillars between the four eyespot/colour treatments $(P$. tremuloides: overall Wald $=14.67, d f=3, N=320, P=$ 0.002 ; remaining $P>0.38$, see Supplementary Material for details). For those pastry caterpillars on $P$. tremuloides, a significant eyespot $\times$ colour interaction contributed to the overall significance $($ Wald $=-3.77, P<0.001)$. Pairwise treatment comparisons revealed the same directionality in survivorship as the full analysis but they showed greater strength (Fig. 2-2b). Notably, the artificial caterpillars pinned to $P$. tremuloides experienced a relatively high baseline hazard rate (appendix B Fig. S2-3). 


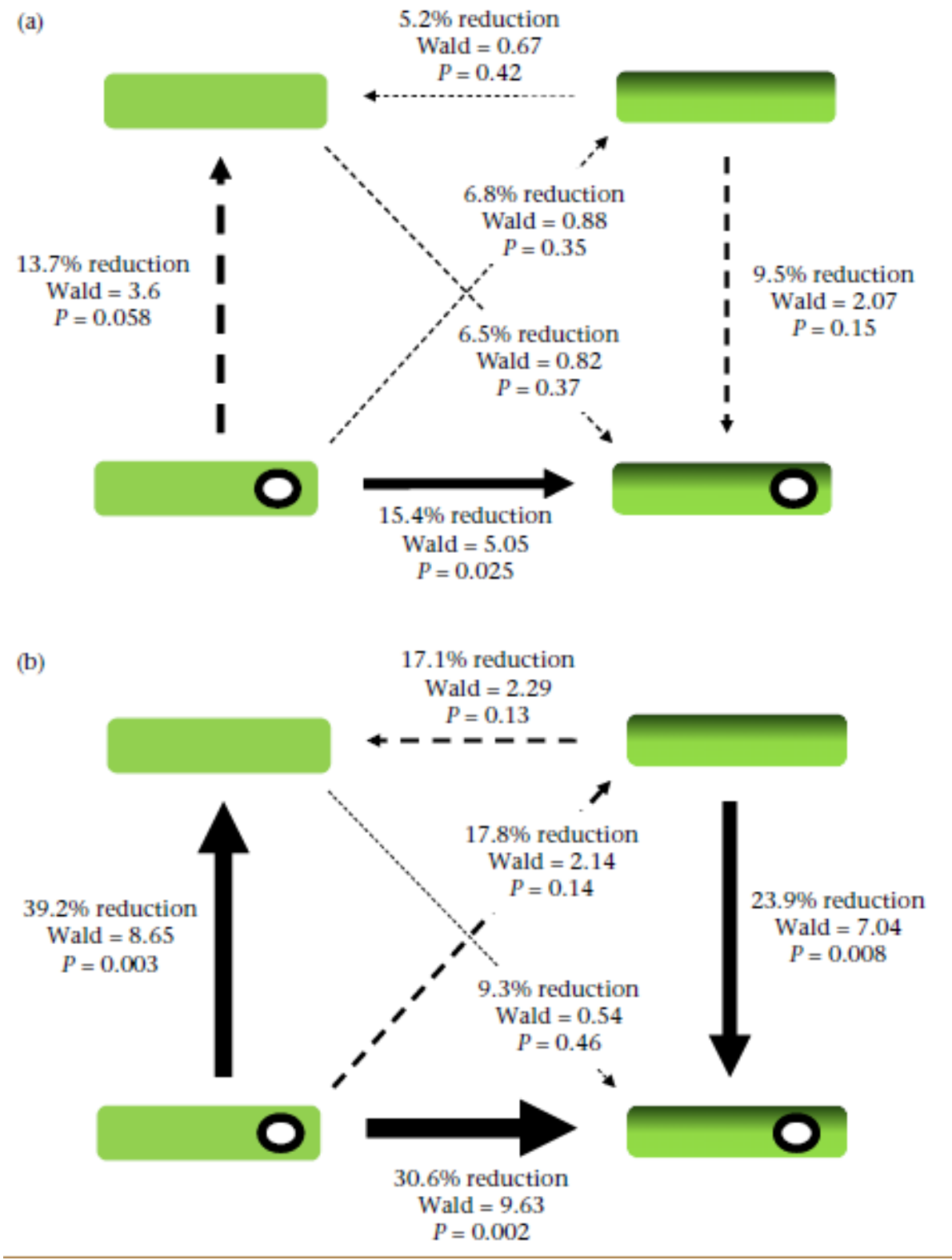

Figure 2-2: (a) Results from pairwise Cox proportional hazard regression comparisons of pastry caterpillar survival stratified by tree species and location, and clustered by the tree to which pastry caterpillars were pinned. Eyespot (no eyespots versus eyespots present) and colour (solid green versus countershaded) treatments were arranged in a $2 \times 2$ factorial design. (b) Results from the same analyses conducted using only those pastry caterpillars pinned to Populus tremuloides (primary host of Papilio canadensis; Scriber et al. 1995). In both panels, arrow width is proportional to the relevant Wald statistic and points towards the treatment with lower hazard rate. Solid arrows are significant at $P<0.05$. 
Although many prey items presumed attacked had been fully removed by predators $(52 \%, 334 / 642)$, the remaining attacked baits were recovered from the field (48\% recovered, 308/642). Overall, a similar proportion of caterpillars disappeared upon attack in all four treatments (mean proportion $\pm \mathrm{SE}=0.50 \pm 0.05 ; F_{3,24}=1.27, P=0.31$ ). There was no significant effect of treatment on the number of pecks received by attacked pastry caterpillars recovered from the field $\left(F_{3,307}=1.12, P=0.295\right.$, overall mean $\pm \mathrm{SE}=$ $3.78 \pm 0.18)$. Pecks were not evenly allocated along all sections of the pastry caterpillars $\left(\chi^{2} 5=459.34, P<0.001\right)$, and the distribution of pecks was affected by the presence of eyespots $\left(\chi^{2}{ }_{6}=49.858, P<0.001\right)$, but not by countershading $\left(\chi^{2} 6=9.904, P=0.13\right)$. Results from model comparison using AIC confirmed these results (appendix B Table S2-4). Specifically, prey with eyespots were pecked less in the section anterior of the eyespot $(z=-3.699, P<0.001)$ and pecked more frequently within the eyespot section $(z$ $=4.406, P<0.001)$ and on the posterior tip furthest from the eyespots $(z=5.091, P<$ 0.001) than prey without eyespots (Fig. 2-3, appendix B Table S2-5). The middle sections of prey with and without eyespots rarely received pecks (Fig. 2-3). Therefore, pecks were not distributed evenly along the bodies of any caterpillar type, but eyespots affected where birds pecked the prey. 


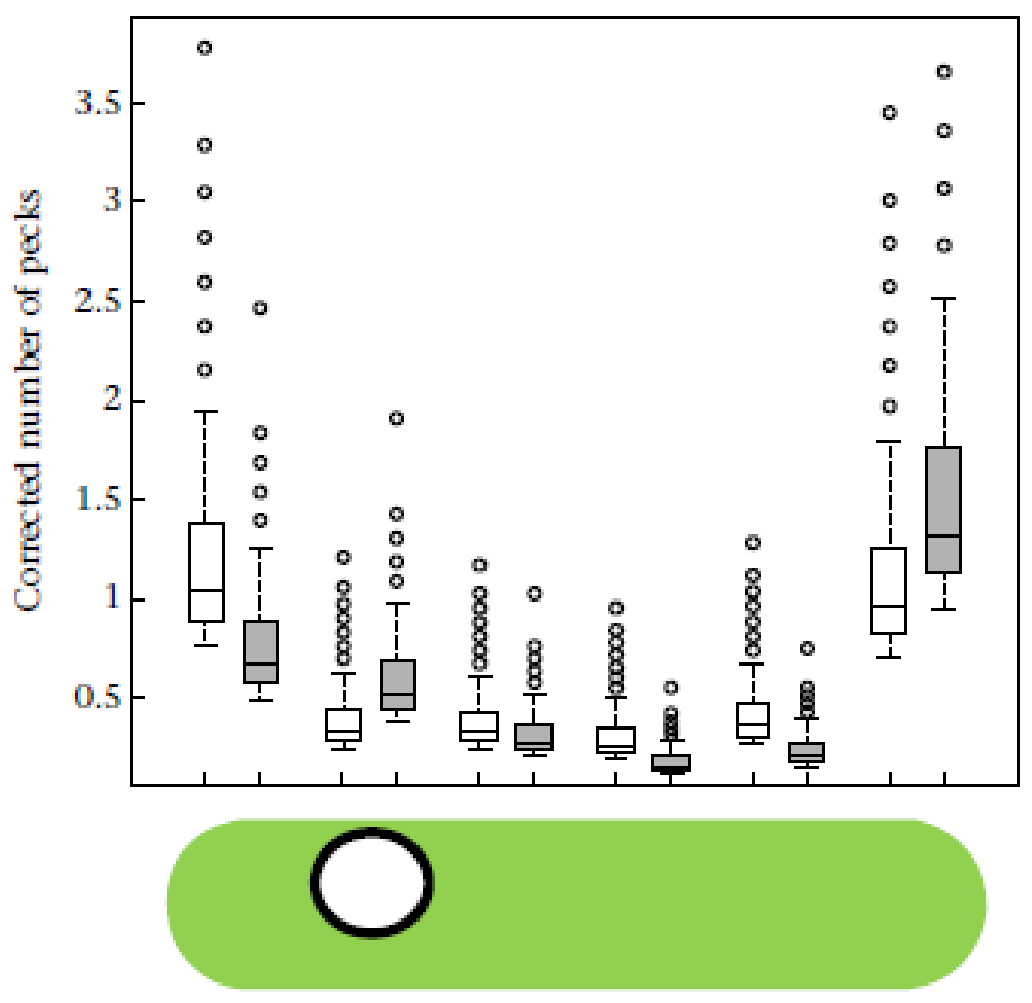

Figure 2-3: Box plot depicting the number and location of peck attacks on pastry caterpillars deployed in the field after controlling for the lack of independence of multiple pecks on a given individual prey item. White boxes: no-eyespot treatment; grey boxes: eyespot treatment. The corrected median number of pecks is depicted by the bar inside the box, hinges indicate the $25 \%$ and $75 \%$ quartiles, and whiskers extend to 1.5 times the interquartile range, with values outside of this range depicted as open circles. 


\subsection{Discussion}

Our experiment examined the protective value of eyespots and countershading in caterpillar-like prey subject to predation by wild avian predators. Surprisingly, we did not observe a strong main effect of eyespots or countershading on survival. However, a significant interaction indicated that countershading and eyespots act synergistically to reduce predation. Specifically, we found that eyespots increased the survival of countershaded prey but reduced survival of solid prey. These patterns were more apparent when analyses were restricted to include only prey pinned on trembling aspen (Populus tremuloides), the primary host of Pa. canadensis (Scriber et al. 1995). Finally, pecks were not randomly or symmetrically distributed on eyespot caterpillars, indicating that eyespots may also affect where predators strike the prey. To our knowledge this is the first experiment to demonstrate that eyespots can protect caterpillar prey from predation by wild birds in the field, albeit to a limited extent.

If eyespots do protect caterpillars through predator eye mimicry, then the weak avoidance of eyespot caterpillars may be a result of our design necessarily excluding relevant caterpillar behaviours. Such behaviours have long been recognized in caterpillars with eyespots (Bates 1862; Poulton 1890) and also occur in Papilio sp. caterpillars (T. J. Hossie, personal observation). The extent to which variation in behaviour and morphology influence predation of eyespot caterpillars by avian predators is currently being evaluated. The diversity of eyespots observed in the natural world, many of which only vaguely resemble eyes, suggests that eyespots need not closely resemble a 
predator's eyes; however, it is conceivable that the reduced efficacy of eyespots that we observed may also have been the result of our eyespot design.

Countershaded prey without eyespots in our study did not have greater survival than solid green prey. This contrasts with previous work (Edmunds \& Dewhirst 1994; Rowland et al. 2007, 2008), although a protective effect of countershading has not always been observed (Ruxton et al. 2004b; Speed et al. 2004). It is possible that microhabitat choices of prey strongly influence the efficacy of countershading, or that the bird community in our study areas may have been composed of species where countershading does not affect prey detection (e.g. Speed et al. 2004). Countershading might have been more effective if caterpillars had been deployed on leaves (see also: Speed et al. 2004), yet Rowland et al. (2008) observed a protective effect of countershading for green prey similarly deployed on branches. Instead, we suspect that countershading may be insufficient for our larger-bodied prey positioned on branches as the prey used in our experiment were also larger than those used in previous studies (e.g. Rowland et al. 2008). A limitation of our design is that our solid treatment was the same colour as the ventral, rather than dorsal, side of the countershaded prey, meaning that treatments would have been seen as different colours when viewed from above. As we observed no significant main effect of prey body colour, this constraint most likely had little effect on our results; nevertheless, the design of our body colour treatment may not adequately distinguish between the effect of background matching and countershading.

A key result of our study is that eyespots reduced the hazard to countershaded prey but not solid prey. Thus, the protective value of conspicuous spots most likely depends on other aspects of prey coloration (see also, Stevens et al. 2008c). One possible 
reason for this interactive effect is that combining eyespots with countershading makes the caterpillar a more convincing snake mimic, perhaps by acting additively as a cue signaling danger, as many arboreal snakes are also countershaded. Indeed, birds may use patterns of dangerous snakes that function in crypsis (e.g. the zigzag pattern of Vipera sp.) as avoidance cues (Wüster et al. 2004; Valkonen et al. 2011b). Whether the observed strong effects on Po. tremuloides are explained by the difference in sample size relative to other tree species, or by a specific biological factor remains unclear, and future work should investigate whether eyespots interact with countershading specifically or body colour more generally. Regardless of the mechanism, our results indicate that the effect of eyespots is not independent of habitat or additional aspects of prey coloration, emphasizing the epistatic effects of different traits on fitness.

Eyespots also affected the area of the pastry caterpillar's body that was pecked by birds, which may represent an additional advantage to the caterpillars. When eyespots were lacking, pecks were symmetrically directed towards the terminal sections; however, when eyespots were present, the terminal section anterior of the eyespot received relatively few pecks. In addition, both the section with eyespots and the terminal section furthest from the eyespots were pecked more than the comparable positions on caterpillars without eyespots (Fig. 2-3). Thus, while some avian predators may target the eyespots upon attack, others may be intimidated from attacking the end of the caterpillar with eyespots. Still other birds probably do not fear eyespots as eyespot prey were occasionally removed completely. Blest (1957) found that birds were more likely to peck towards a single spot painted on mealworm prey, and our finding that pecks were directed towards eyespots is consistent with his suggestion that eyespots may direct 
strikes towards the osmeterium in papilionid larvae. Whether such change in the distribution of pecks increases caterpillar survival remains unknown; however, fewer pecks anterior of the eyespots should minimize the change of damage to a caterpillar's true head, located anterior to the eyespots in many eyespot caterpillars including $P a$. canadensis.

Overall, our work is consistent with the proposed intimidation function for caterpillar eyespots, but many questions remain to be addressed empirically, namely: (1) do caterpillar eyespots represent a form of mimicry, (2) do tropical migratory birds carry either learned or innate aversions towards eye-like features to temperate areas, and (3) what selection pressures favour eyespots over other defences? Although challenging, addressing these questions will help us understand the widespread phenomenon of eyespots and may provide new insights into the evolution of mimicry.

\subsection{Acknowledgements}

S. Adamo and two anonymous referees provided helpful comments on this manuscript. We thank H. Kienzle for help during the implementation of this work, as well as B. and E. Hossie, the University of Guelph Kemptville Campus, and the Ferguson Forest Centre for allowing use of their property. L. Touzin helped facilitate work on crown land. This work was funded through a Natural Sciences and Engineering Research Council of Canada Discovery grant awarded to T.N.S. 


\section{Chapter: Defensive posture and eyespots deter avian predators from attacking caterpillar-like prey}

Thomas J. Hossie ${ }^{1 *}$ and Thomas N. Sherratt ${ }^{1}$

${ }^{1}$ Department of Biology, Carleton University, Ottawa, ON, Canada

*Corresponding author

Published in Animal Behaviour (2013) 86: 383-389

Reproduced in whole in accordance with Elsevier terms and conditions. 


\subsection{Abstract}

Many lepidopteran caterpillars appear to gain protection from insect-eating birds because they possess eyespots; a pair of conspicuous markings on the body which are generally thought to resemble the eyes of a dangerous predator. Similarly, many caterpillars widen their anterior body segments when threatened, and this defensive posture may also deter attack because it forms what resembles the triangular head of a dangerous viper. Yet it is unclear whether this putative head-shape mimicry alone can protect caterpillar prey, or whether a modified head-shape enhances the protection afforded by eyespots. To disentangle the protective value of eyespots and head-shape mimicry we created four types of artificial caterpillar in a $2 \times 2$ factorial design and deployed them on tree branches in the field, where they were exposed to predation by wild birds. "Survivorship" of the artificial caterpillars was monitored over $90 \mathrm{~h}$ in six locations. We found a clear survival advantage for prey that possessed either eyespots or a widened head-shape. However, prey possessing both eyespots and the widened head did not survive longer than prey with either defence alone, suggesting that the traits did not act additively or synergistically to deter attack. Interestingly though, only caterpillars in the eyespot-defensive posture treatment received more pecks directed towards the anterior "head" region. To our knowledge this is the first study to examine the interaction between eyespots and head-shape mimicry as means of anti-predator defence in caterpillar prey, and demonstrates clearly the protective advantage of these traits in caterpillar prey subject to predation by wild birds. 


\subsection{Introduction}

The caterpillars of many lepidopteran species possess a pair of conspicuous eyespots. These eye-like markings are thought to protect caterpillars because they resemble the eyes of a dangerous predator and thereby deter the attacks of insect-eating birds (Janzen et al. 2010). To human eyes, the eyespots do not always confer a close resemblance to any given model, but sometimes the extent of mimicry is compelling. Indeed, Darwin's contemporary, Henry Walter Bates, described his encounter with one particular species of snake-mimicking caterpillar as "The most extraordinary instance of imitation that I ever met... [the caterpillar] startled me by its resemblance to a small Snake..." (Bates 1862, p 509).

Recently we provided the first empirical field demonstration that eyespots may provide caterpillars with protection from avian predators (Hossie \& Sherratt 2012). Yet, eyespots do not occur independently of other traits that influence their protective value. For example, our study showed that other aspects of prey body colour (i.e., countershading) affect how well eyespots deter avian attack (Hossie \& Sherratt 2012). Another trait that may influence the protective value of eyespots is the characteristic defensive posture that many of these caterpillars adopt when threatened. Specifically, the defensive posture typically involves widening the anterior body segments near the eyespots and may enhance their overall resemblance to enemies of insect-eating birds such as Viperidae snakes (Fig 3-1a,c; Valkonen et al. 2011). Bates observed this first hand: "The first three segments behind the head were dilatable at the will of the insect, and had on each side a large black pupillated spot, which resembled the eye of the reptile: it was a poisonous or viperine species mimicked, and not an innocuous or 
colubrine Snake..." (Bates 1862, p509). To our knowledge, the extent to which this defensive posture enhances the protection afforded by eyespots has never been examined empirically.

Among large caterpillars the facultative widening of anterior body segments when threatened is relatively common, and even occurs in some species that lack eyespots (e.g. Eumopha phorbas, Papilio cresphontes). In those species where the caterpillars do have eyespots, expanding the anterior body segments does produce the appearance of a dangerous snake at least to human observers (e.g., Fig 3-1a). Recent work has also revealed that birds are less likely to attack snake-like prey with triangular "viper-shaped" heads, as compared to prey with a narrower "colubrid-shaped" head (Valkonen et al. 2011a). Indeed, several "non-venomous" colubrid snakes behaviourally modify their head shape when threatened, a process known as "head triangulation", which is generally thought to increase mimetic resemblance to vipers (Werner \& Frankenberg 1982; Werner 1985, 1986). Thus, it seems likely that inflation of the anterior body segments by caterpillars represents a similar form of behavioural mimicry of a specific or generalized threat, and there are countless examples of lepidopteran species whose larvae possess both eyespots and this behaviour (e.g. Hemeroplanes ornatus, Madoryx oiclus, Papilio troilus). Crucially, the distribution of these prey need not overlap with the distribution of their model (e.g., arboreal vipers) to gain protection so long as insect-eating birds have an inherited predisposition to avoid objects with these traits, or if the birds migrate from 
tropical wintering grounds where these models occur (see Janzen et al. 2010, Hossie et al 2012).

The results of Valkonen et al. (2011) are of further interest because they found that both the viper head-shape and dorsal zigzag pattern reduced predation, but did so independently. Similarly, both eyespots and the defensive posture (i.e., head-shape mimicry) may reduce attacks on caterpillars, but do so independently. However, since all snakes have eyes, but not all dangerous snakes have a dorsal patterning, combining headshape mimicry with eyespots might produce a more reliable set of mimetic signals. Thus head-shape mimicry is perhaps more likely to act additively (or synergistically) with eyespots than with a dorsal zigzag pattern in protecting mimetic prey.

To date only a few anecdotal reports have indicated that caterpillars may effectively deter avian predators by combining eyespots and behaviour. Specifically, Poulton (1890) describes the defensive posture of the Deilephila elpenor and D. porcellus caterpillars (which have eyespots), as well as the results from unreplicated staged presentations using these caterpillars (Poulton 1890). Briefly, these tests indicated no protection from a tame jay, weak avoidance of the caterpillar by domestic foul, and the deterrence of small birds (e.g., sparrows) from a feeding tray containing seed or bread crumbs (Poulton 1890). Additionally, Shirota (1980) observed that 6 captive starlings (Sturnus cineraceus) feared, and did not attack, live final instar Deilephlia elpenor lewisii caterpillars within 3 minutes. Together these mixed results suggest that the eyespots and the defensive posture may deter an attacking bird, yet no study has attempted to disentangle the independent or interactive effects of these traits on protection. Clearly well-replicated field studies are required to determine the true protective value of these 
traits. To examine the protective value of eyespots and behavioural mimicry (i.e., facultative widening of anterior body segments) in caterpillars we deployed artificial caterpillars in the field and monitored their 'survival'. Treatments were arranged in a $2 \times 2$ factorial design which allowed us to determine i) if either eyespots or head-shape mimicry can effectively protect caterpillars from avian predators, and ii) whether combining these defences further increases their ability to deter attack. 


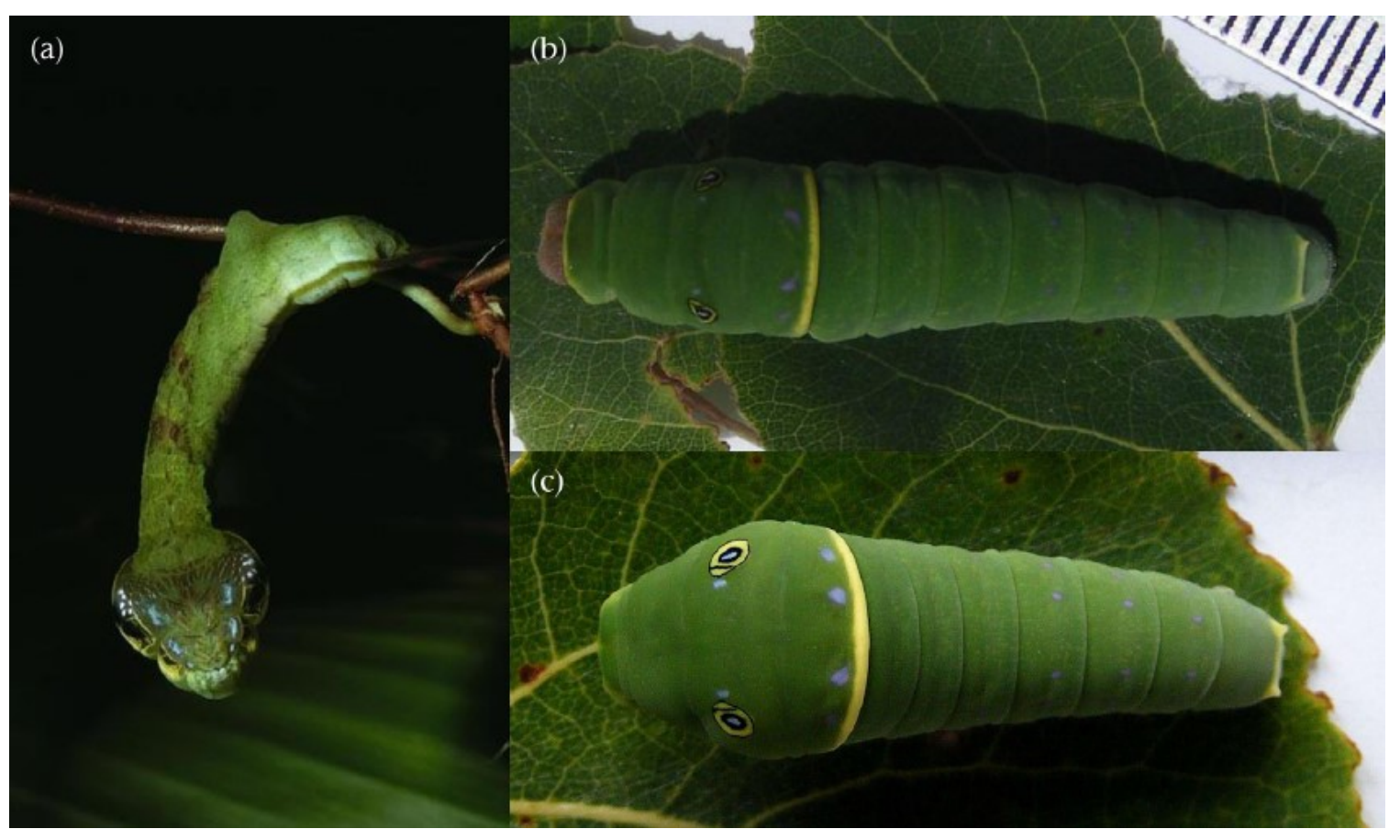

Figure 3-1: (a) Penultimate instar of a Costa Rican Hemeroplanes triptolemus (Sphingidae) caterpillar displaying its defensive posture; what appears to be the dorsal side of the snake head is in fact the ventral side of the caterpillar thorax. Photo is from Janzen and Hallwachs (2014), specimen number 03-SRNP11366 (used with permission). (b) Ultimate instar Papilio canadensis caterpillar in resting posture, and (c) ultimate instar Papilio canadensis caterpillar in defensive posture (photos by TJH). 


\subsection{Methods}

Artificial Prey

Artificial caterpillars were designed to assess whether eyespots and adopting a defensive posture (i.e., thickening of the anterior body segments) can protect caterpillars from attack by wild birds. Manufacture of artificial caterpillars followed methodology similar to previous studies (e.g., Church et al. 1997; Rowland et al. 2008), and for ecological relevance our models were based loosely on the late instars of Papilio canadensis and P. glaucus caterpillars. A number of species from the Papilionidae genus Papilio have caterpillars with conspicuous eyespots that facultatively adopt this defensive posture when threatened. Specifically, these caterpillars curl their true head into their body while inflating their anterior body segments (Figure 3-1b,c; T. Hossie, personal obs.).

Pastry caterpillars conformed to 4 treatments: i) no eyespots-rest posture, ii) no eyespots-defensive posture, iii) eyespots-rest posture, iv) eyespots-defensive posture. We have shown previously that eyespots effectively protected artificial caterpillars from bird attacks only when the caterpillars were also countershaded (Hossie \& Sherratt 2012) as commonly observed (de Ruiter 1956, Lederhouse 1990). We thus decided to make all treatments similarly countershaded. Briefly, pastry caterpillars were manufactured with a 3:1 mixture of white flour (White Rose ${ }^{\mathrm{TM}}$ ) and lard (Tenderflake ${ }^{\mathrm{TM}}$ ). From this, light green pastry was produced by adding 3 drops of Leaf Green AmeriColor ${ }^{\mathrm{TM}}$ Soft Gel Paste $^{\mathrm{TM}}$ and $20 \mathrm{ml}$ of water, whereas 6 drops of gel paste and $5 \mathrm{ml}$ of water were added to produce the dark pastry. Dark and light pastry was rolled to $0.5 \mathrm{~cm}$ diameter strips which were then cut into $4 \mathrm{~cm}$ lengths. Two-toned (i.e., countershaded) prey were then created 
by pressing together one piece of dark and light pastry such that the final artificial prey were cylinders $4 \mathrm{~cm}$ long with a diameter of $\sim 0.7 \mathrm{~cm}$. For our purposes the dark side was considered dorsal as prey were presented on the upper side of branches. To create prey with a modified "head" shape, representing the caterpillar's defensive posture, half of these two-toned models were pressed into a mould made of Plaster of Paris (The Webster Group Inc.). This process created models that were equivalent to the un-moulded prey in volume and colour, but were $3 \mathrm{~cm}$ in length and asymmetrically widened on one end (Figure 3-2b, d). The eyespot design was modified from Hossie and Sherratt (2012) such that here the spots they were slightly smaller and had a central bright iris, so as to be more representative of the eyespots on Papilio caterpillars. We used Elmer's ${ }^{\mathrm{TM}}$ non-toxic Tempera Paint to make eyespot treatments. First two yellow circles $(\sim 0.45 \mathrm{~cm}$ in diameter, 1:1 mix of yellow and white paint) were painted dorso-laterally on the prey, 0.4 cm apart. Rest- and defensive-posture treatments were different lengths (i.e., 4 and $3 \mathrm{~cm}$, respectively), thus we painted eyespots $\sim 0.7 \mathrm{~cm}$ from the anterior tip for "rest posture" models, but only $\sim 0.5 \mathrm{~cm}$ from the anterior tip of "defensive posture" models to maintain roughly equivalent proportional distance to the anterior end. Excess yellow paint was removed from the centre of the spots using a thin paintbrush and a $0.3 \mathrm{~cm}$ black circle was painted inside each. Finally, a small white spot of paint $\sim 0.1 \mathrm{~cm}$ in diameter was added to the center of the black circle. A dorsal view of an eyespot caterpillar is provided in Figure S3-1. Finished pastry caterpillars (e.g., Figure 3-2) were checked against a standard model prey for each treatment to ensure uniformity in size and shape of the body, as well as the size and position of eyespots. 


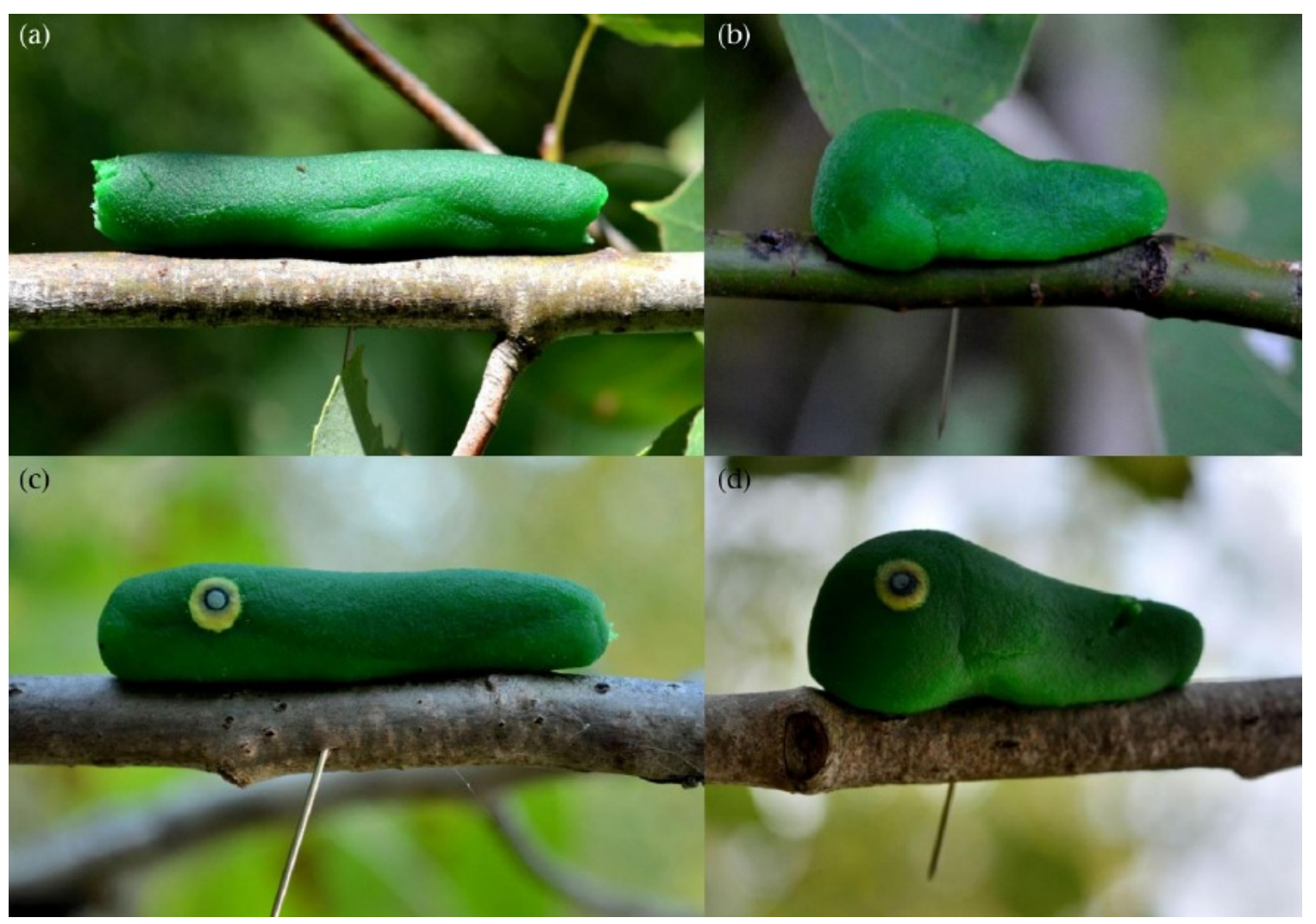

Figure 3-2: Examples of pecked pastry caterpillars from each of the four treatments used in this experiment. (a) no eyespots-resting posture; (b) no eyespots-defensive posture; (c) eyespots-resting posture; (d) eyespots-defensive posture. 


\section{Site description}

Field trials were conducted near Kemptville (Site 1: 4503’00 N, 75³9’44 W;

Site 2: 4459’48 N, 75³8’22 W) and Oxford Mills (Site 3: 445627 N, 75³8’56 W), Ontario, Canada. Experiments were run between June 14 and August 312012 which overlaps with the dates when eyespotted Papilio canadensis (Figure 3-1b,c) and $P$. glaucus caterpillars can be found in the wild in our study area (T. Hossie, personal obs.; Hall et al. 1996). All three sites were composed of secondary growth mixed-deciduous forest. Each site was no closer than $5.5 \mathrm{~km}$ from another site. Preliminary scouting of each site confirmed the presence of $P$. canadensis adults and several species of insectivorous birds. Insectivorous bird species observed in each of the field locations are detailed in appendix Table S3-1.

Prey presentation

Pastry caterpillars were deployed between 1600-1900h on the first day of a replicate site. Specifically, 24 trees were selected along a linear transect ( $\sim 10 \mathrm{~m}$ apart), and to each tree a single artificial prey was pinned on the upper side of a live branch in the north, south, east and west zone of a tree. Trees were restricted to one of 4 species (Populus tremuloides, Populus deltoides, Betula papyrifera, Acer rubrum, Table S3-2) which have all been identified as a host species of "snake mimic" Papilio caterpillars (Scott 1986; Scriber et al. 1995; Allen et al. 2005). Each tree received a single pastry caterpillar from each treatment and branch zone (i.e., N, E, S, W) was allocated randomly. Attaching 4 prey to each tree has the benefit of reduced variation in microhabitat among treatments. Selected branches were between 1-2 m from the ground 
and were roughly $0.5-1 \mathrm{~cm}$ in diameter. Pastry caterpillars were considered killed if they were no longer present or had been attacked by a bird (peck marks present; e.g., Figure 32, Figure S3-1). 'Survival' was checked at 14, 19, 24, 38, 43, 48, 62, 67, 74, and 90 hours from deployment. In all cases of missing baits, the pin (invariably still stuck in the tree branch) was found and the ground underneath was searched for the bait or any remaining pieces of an attacked bait. All attacked prey were photographed then removed. Artificial prey damaged by rain, slugs or ants were removed $(n=111)$ and considered censored (i.e., considered to have survived only to that time period, but not considered killed) in the final analysis. After $90 \mathrm{~h}$, remaining prey were removed.

The entire process was replicated 6 times (two times per site, albeit at a different location within site) for a total of 576 artificial prey deployed. Each consecutive replicate was conducted in an entirely new location within one of the 3 larger sites, and was at least $200 \mathrm{~m}$ from any previous location within that site. To avoid temporal confounds among sites, consecutive replicates were always conducted at a different site in a systematic order (i.e., Site 1 location 1, Site 2 location 1, Site 3 location 1, Site 1 location 2 etc.).

Analyses

We analyzed the survival of artificial prey using Cox proportional hazards regression (Cox 1972), which can handle both censored data and non-uniform changes in predation risk. Since pastry caterpillars were pinned singly on branches, individual caterpillars may well have been encountered independently on the trees (e.g., Rowland et al. 2008); however we controlled the possible lack of independence between baits on the 
same tree by clustering the baits by tree in our analysis. Overall significance of the model was assessed using the Wald test which does not assume independence of observations within a cluster. Factors in our model included Head-shape (i.e., rest vs. defensive posture), Eyespot (i.e., eyespots vs. no eyespots), and the Head Shape $\times$ Eyespot interaction. The effects of location and tree species were not of direct interest, and we did not have a priori hypotheses regarding their effect on survival. However preliminary analysis indicated that the hazard rate differed among tree species and locations (see Figures S2-S3). We therefore stratified the analysis to permit location- and tree speciesspecific baseline hazard rates. This analysis was conducted in R (R Development Core Team 2014) using the survival library (Therneau 2014), and the assumption of proportionality was tested using the cox.zph function.

We quantified the number and location of bird pecks on pastry caterpillars by analysing digital photographs of all retrieved baits that showed any evidence of being attacked. For each pastry caterpillar with eyespots we counted the number of pecks in each of 6 even-sized continuous sections: anterior tip, body section with eyespots, midanterior body, mid-posterior body, hind-mid posterior body, and posterior tip. For the no eyespots-rest posture treatment we counted the number of pecks in each body section, with the anterior-posterior designation assigned randomly by a coin toss. To determine if the number of pecks differed among body sections and among treatments we used a generalized mixed effects model with a Poisson error distribution and log link function. We included section as a fixed factor and set individual as a random factor to control for the lack of independence of multiple pecks on a given caterpillar. The significance of a factor was examined by comparing models with and without the parameter of interest 
using a likelihood ratio test. We also compared the fit of our models using Akaike's information criterion (AIC). These analyses were conducted in R (R Development Core Team 2014) using the lmer 4 package (Bates et al. 2011).

\subsection{Results}

The total $90 \mathrm{~h}$ mortality rate for a location ranged from $28 \%$ to $89 \%$ (mean \pm SE: $63.6 \pm 9.12 \%$ ). Both the main effects of Head Shape, and Eyespots significantly reduced the hazard rate $(z=-2.65, P=0.008 ; z=-1.98, P=0.048$, respectively), however we did not detect a significant Head Shape $\times$ Eyespot interaction $(z=1.72, P=0.086)$. The modified Head Shape reduced hazard by $23.6 \pm 10.1 \%$, whereas possessing Eyespots reduced hazard by $18.5 \pm 10.4 \%$ (Table 3-1). To assess the relative survival rates of the 4 treatments we conducted pair-wise comparisons of the 4 treatments (Figure 3-3). This analysis revealed that the no eyespots-rest position treatment suffered the highest predation rate, with comparatively lower hazard arising in the no eyespot-defensive posture $(22.1 \pm 9.6 \%$ reduction, Wald $=6.84, P=0.009)$, and the eyespot-defensive posture treatments $(21.1 \pm 10.9 \%$ reduction, Wald $=4.75, P=0.029$, Figure $3-3)$. The reduction in hazard experienced in the eyespot-rest posture treatment relative to no eyespots-rest position prey was near significant $(17 \pm 10.4 \%$ reduction, Wald $=3.63, P=$ 0.057, Figure 3-3). Classifying only prey recovered with peck marks as killed (i.e., considering all missing prey as censored) produced qualitatively similar results (Table S3-3). 
Table 3-1: Results from fitting Cox proportional hazards regression model of pastry caterpillar survival stratified by location and tree species.

\begin{tabular}{lllllll}
\hline & Coef & Exp(coef) & SE(coef) & Robust SE & $\boldsymbol{z}$ & $\boldsymbol{P}$ \\
\hline Eyespots & -0.2049 & 0.8147 & 0.1499 & 0.1037 & -1.976 & 0.0481 \\
Head Shape & -0.2687 & 0.7644 & 0.1469 & 0.1014 & -2.65 & 0.00805 \\
Eyespots $\times$ Head Shape & 0.2274 & 1.2553 & 0.2129 & 0.1326 & 1.715 & 0.0864 \\
\hline
\end{tabular}

Parameter estimates and associated significance tests depict how eyespots and posture affect survival of pastry caterpillars. Since fates of pastry caterpillars that were pinned to the same tree were not necessarily entirely independent, we clustered caterpillars that were pinned to the same tree in the analysis. In our analysis we used a binary coding system (i.e., $0 / 1$ ) for our factors indicating the absence/presence of eyespots or a widened head shape. 


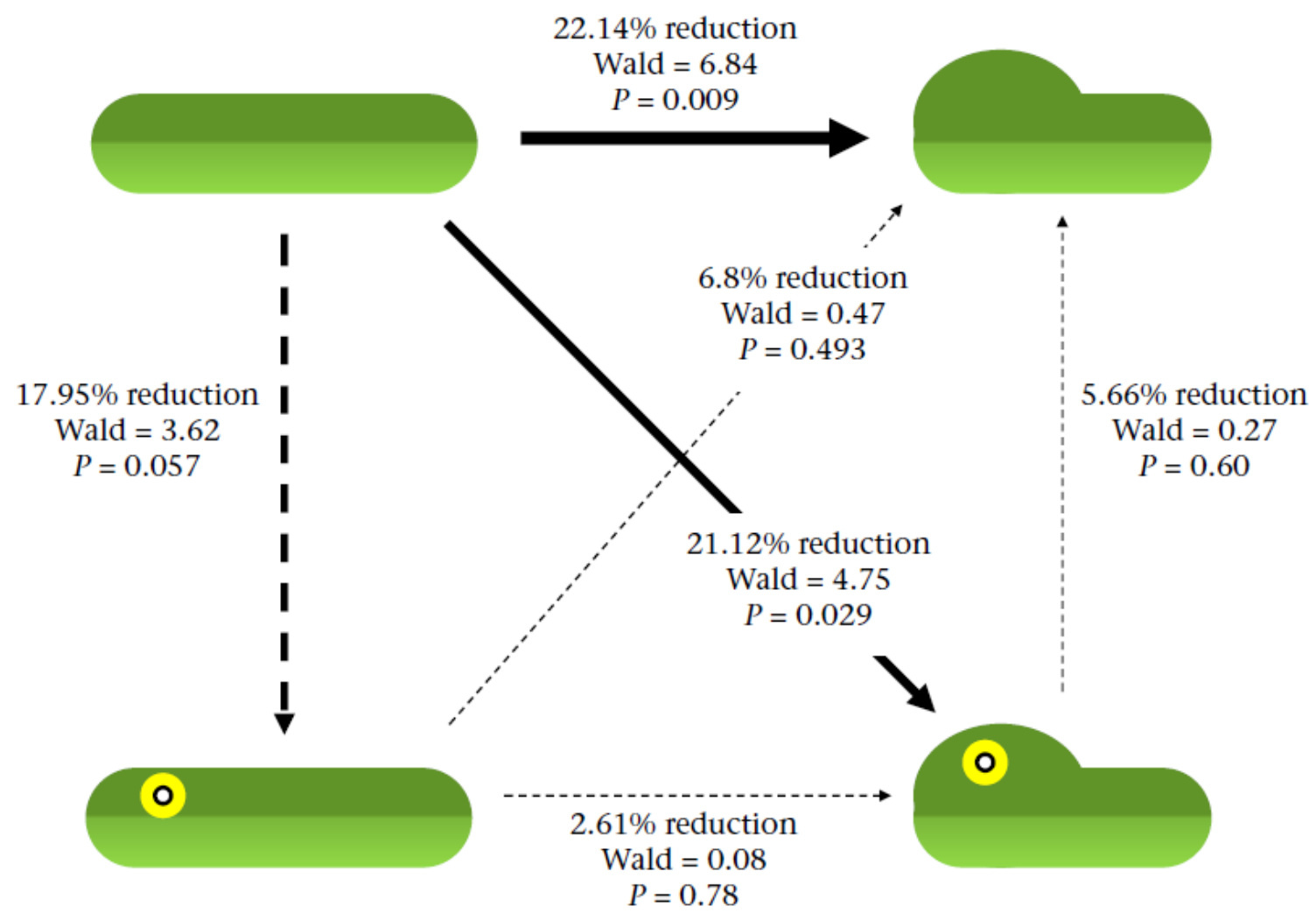

Figure 3-3: Results from pairwise Cox proportional hazard regression comparisons of pastry caterpillar survival stratified by tree species and location, and clustered by the tree to which pastry caterpillars were pinned. Eyespot (i.e., no eyespots versus eyespots present) and Head-Shape (i.e., resting posture versus defensive posture) treatments were arranged in a $2 \times 2$ factorial design. Arrow width is proportional to the relevant Wald statistic and points towards the treatment with lower hazard rate. Solid arrows are significant at $\mathrm{P}<0.05$. 
Of the 364 prey assumed killed, we recovered $185(50.8 \%)$ that had been pecked by birds in the field. Overall, a similar proportion of caterpillars disappeared upon attack in all 4 treatments (mean proportion $\pm \mathrm{SE}=0.48 \pm 0.05, F_{3,20}=0.486, P=0.70$ ). However, the total number of pecks on each bait was affected by both eyespots (mean number of pecks \pm SE: no eyespots $=4.39 \pm 0.38$, eyespots $=6.13 \pm 0.57 ; F_{1,181}=8.26, P$ $=0.005)$, and head shape $\left(\right.$ rest $=4.31 \pm 0.34$, defensive $=6.25 \pm 0.60 ; F_{1,181}=6.51, P=$ $0.012)$, but not the eyespot $\times$ head shape interaction $\left(F_{1,181}=0.02, P=0.88\right)$. More pecks were directed towards the section with eyespots in the defensive posture prey when compared to the equidistant position at the other end (i.e., section $5, z=3.119, P=$ 0.002), and when compared to the same position (i.e., section 2) on defensive-posture prey lacking eyespots $(z=3.194, P=0.001$, Figure $3-4)$. Similarly, the adjacent section (i.e., section 3) in defensive-posture prey also received more pecks if the prey had eyespots $(z=2.603, P=0.009)$. The pecks were distributed symmetrically in the remaining 3 treatments (all $P>0.06$, Table S3-4) with most pecks directed towards the tips and relatively few pecks directed toward the middle sections (Figure 3-4). Therefore eyespots affected where birds pecked the artificial caterpillars only when they were in their defensive posture and not when prey were in the rest-posture. 

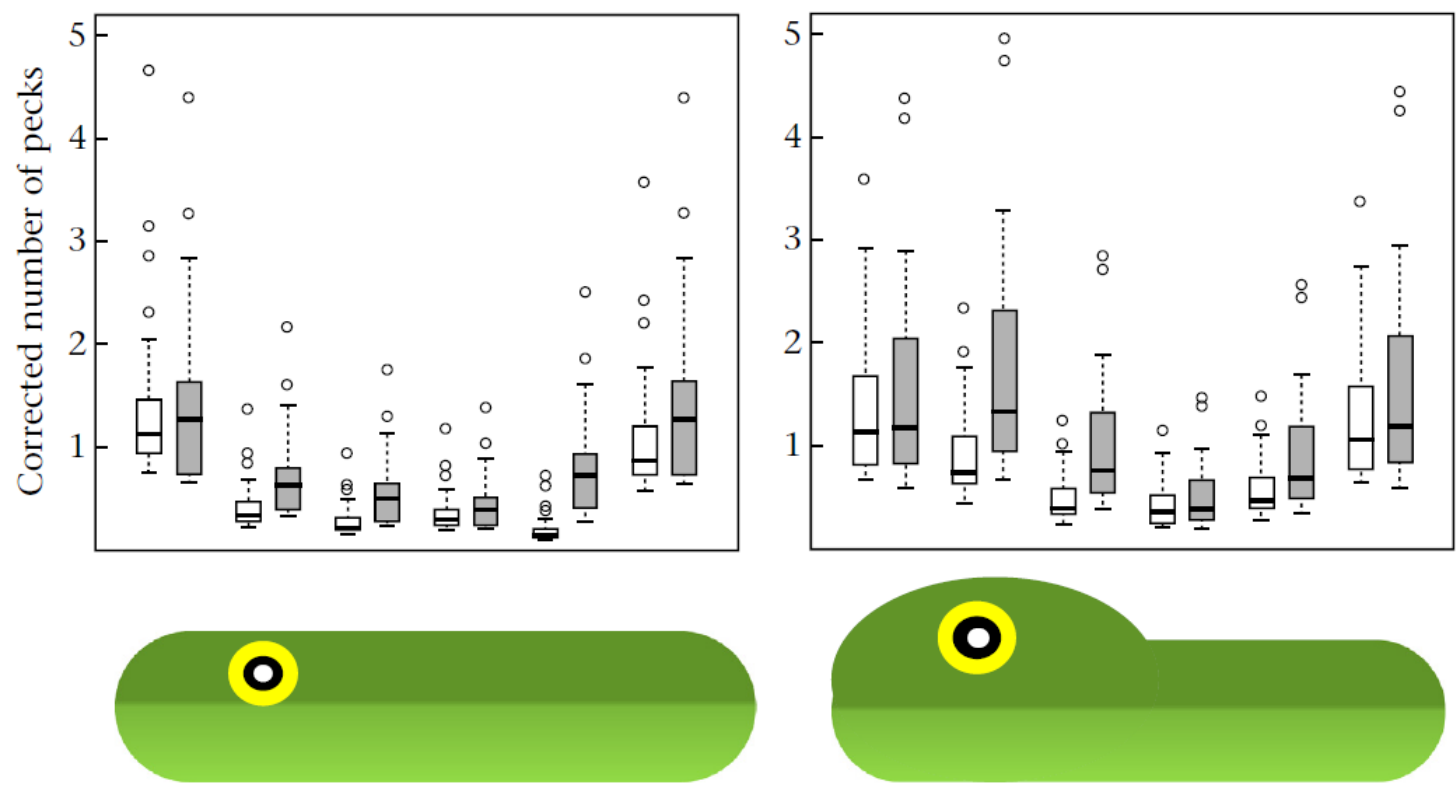

Figure 3-4: Box plot depicting the median number and location of peck attacks on pastry caterpillars deployed in the field after controlling for the lack of independence of multiple pecks on a given individual prey item. White boxes: no-eyespot treatment; grey boxes: eyespot treatment. The corrected median number of pecks is depicted by the bar inside the box, hinges indicate the $25 \%$ and $75 \%$ quartiles, and whiskers extend to 1.5 times the interquartile range, with values outside of this range depicted as open circles. 


\subsection{Discussion}

Our experiment examined the protective value of eyespots and a widened headshape in caterpillar-like prey subjected to predation from wild avian predators. We found a clear survival advantage for caterpillar targets that possessed either, or both, eyespots and a widened head-shape. Although we had expected to observe a significant interactive effect between eyespots and head-shape mimicry, we instead found that these defences apparently operate independently to deter attack. Specifically, the treatment possessing both eyespots and the widened head shape did not survive longer than prey with either defence alone. Interestingly though, only caterpillars in the eyespot-defensive posture treatment received more pecks directed towards the anterior "head" region (Figure 3-4). To our knowledge this is the first empirical study to examine the interaction between eyespots and head-shape mimicry as means of anti-predator defence in caterpillar prey, and demonstrates clearly the protective advantage of these traits in caterpillar prey subjected to predation by wild birds.

In this experiment we observed a strong main effect of caterpillar eyespots, which indicated that possessing eyespots conferred a significant survival advantage. The protective value of eyespots observed here is therefore much greater than what we had observed previously (Hossie \& Sherratt 2012). In our previous study the eyespot design was purposefully large and conspicuous in an attempt to augment their possible effect on survival (Hossie \& Sherratt 2012), whereas here we modified the design to be a closer approximation of the eyespots possessed by Papilio caterpillars. Therefore, it is possible that we observed a greater deterrent effect of eyespots in this study because we used a more realistic design. Our revised eyespot design also incorporated a white "sparkle" 
marking in the center of the black pupil (Figure 3-2). Such "sparkle" features have recently been shown to enhance the protective effect of eyespots in adult butterflies (Blut et al. 2012), and may operate similarly in caterpillars. Thus, our work clearly demonstrates that possessing eyespots confers a protective advantage, and we can infer that aspects other than the conspicuousness and size of eyespots critically influence their protective value to caterpillar prey.

Pastry caterpillars with a widened "head" region (i.e., the defensive posture) survived significantly longer than prey in the "rest posture", but this protective effect was not augmented by the presence of eyespots. That is, having either eyespots or a widened "head" protected prey as well as both traits together. This result is consistent with Valkonen et al. (2011) who found that although both viper-like patterning and head shape reduce predation on clay snakes they do not act additively. Interestingly, there are several examples of caterpillars that, despite lacking eyespots, possess a behavioural mimicry response equivalent or superior to closely-related species that possess both traits. For example, Eumorpha phorbas lacks the conspicuous eyespots of the closely related $E$. labruscae, but still produces a convincing viperine head shape when threatened. Similarly, Papilio cresphontes and P. thaos have the thickened "head" area despite lacking the eyespots possessed by the so called "snake mimics" (e.g., P. glaucus, $P$. troilus). This is perhaps additionally indicative that these defensive strategies function reasonably well in isolation. Ongoing research is currently examining the evolutionary history of these defences in Papilionidae and Sphingidae in part to determine whether 
acquiring or losing eyespots secondary best explains the trait combinations observed in modern species.

Peck marks were distributed symmetrically in the rest-posture treatments regardless of eyespot presence, whereas more "head" directed pecks occurred on prey in the defensive-posture particularly when eyespots were also present. Specifically, prey with both a thickened head area and eyespots received significantly more pecks directed at the body segments containing eyespots and the adjacent segment (i.e., sections 2 - 3), compared to either the equidistant sections at the other end (i.e., sections 4 - 5) or sections 2 - 3 on defensive-posture prey lacking eyespots (Figure 3-4). If birds are more likely to attack the eyes or head of a threatening object like a snake, this might indicate that the combination of eyespots and behavioural mimicry does increase the mimetic fidelity beyond either trait alone. In our previous work we found that birds targeted the eyespots even though prey were not in the defensive posture (Hossie \& Sherratt 2012). We suspect that this difference results from changes in the eyespot design between experiments, and additional work should investigate whether such differences in features (e.g., size, composition) determine how an eyespot functions (i.e., deflection vs. intimidation).

This work provides clear evidence for the protective effect of eyespots in caterpillar-like prey. In addition, our experiment demonstrates that a thickened head region also provides a clear protective advantage against attacking birds. The slightly weaker protective effect of eyespots compared to head-shape mimicry $(18.5 \pm 10.4 \% \mathrm{vs}$ $23.6 \pm 10.1 \%$, respectively) might indicate that a diamond-shaped or "viperine" head shape more reliably indicates a true threat than eyespots. Somewhat surprisingly, 
simultaneous possession of both eyespots and the thickened head area did not confer more protection than either trait alone, despite prey with both defenses drawing more "head" directed attacks. It remains unclear as to why prey with both traits did not receive increased protection compared to prey with either trait in isolation. Earlier work has also failed to observe a synergistic effect of eyespots and sound production in the protection of peacock butterflies from avian predators (Vallin et al. 2005). Thus, future research should investigate the learning process of avian predators to develop our understanding of how predators decide which cues reliably indicate danger, and why they sometimes fail to incorporate information from multiple cues when deciding to attack a given prey item. For example, are there adaptive limits to learning the reliability of multiple signals? Such research is likely to help us better understand the evolution of mimetic traits, and may help explain why many mimics only imperfectly resemble their model.

\subsection{Acknowledgements}

We thank H. Kienzle for help during the implementation of this work, as well as B. and E. Hossie, the University of Guelph Kemptville Campus, and the Ferguson Forest Centre for allowing use of their property. L. Touzin helped facilitate work on crown land. D. H. Janzen and W. Hallwachs kindly provided the image of $H$. triptolemus. This work was funded through a Natural Sciences and Engineering Research Council of Canada Discovery grant awarded to T.N.S. 


\section{Chapter: Does defensive posture increase mimetic fidelity of caterpillars with eyespots to their putative snake mimics?}

Thomas J. Hossie ${ }^{1 *}$ and Thomas N. Sherratt ${ }^{1}$

${ }^{1}$ Department of Biology, Carleton University, Ottawa, ON, Canada

*Corresponding author

Published in Current Zoology (2014) 60: 76-89

Reproduced with permission from Current Zoology.

Special Issue on The evolutionary consequences of deception 


\subsection{Abstract}

Organisms often evolve behaviours that increase or reinforce the protection from predators afforded by their morphological defences. For example, mimetic animals may adopt postures or locomotory behaviours that emulate a characteristic feature of their model to increase predator deception. Caterpillars with eyespots are thought to mimic snakes, and when threatened many of these caterpillars adopt a posture that appears to enhance this resemblance. Herein we evaluate the quantitative strength of evidence of behavioural mimicry in the caterpillars of 14 species by comparing how closely a series of putative snake-mimicking caterpillars resemble snakes while at rest and when threatened. Specifically, we quantified the head morphology and eye position of a range of snake species, as well as the shape of the apparent head (i.e. anterior body segments) and position of eyespots in caterpillars resting or in their defensive posture. This allowed us to objectively examine evidence for an increased resemblance to either snakes generally, or to Viperidae snakes specifically, upon adopting the defensive posture. Widening the anterior body segments during the defensive posture typically made caterpillars appear more viper-like as opposed to more snake-like in general. Enhanced resemblance to vipers upon mounting the defensive posture was apparent only from the dorsal view. Laterally, caterpillars more closely resembled snakes in the resting posture and shifting to the defensive posture instead reduced mimetic fidelity. Overall we found evidence for behavioural mimicry in all 14 species examined. We highlight that objectively quantifying mimetic fidelity can help identify key features involved in deception. 


\subsection{Introduction}

Animals show a variety of predator defences ranging from those which minimize detection (crypsis) to those rendering prey both conspicuous and unprofitable to attack (aposematism) (Edmunds 1974; Ruxton et al. 2004a). Each strategy typically involves trait combinations which mutually reinforce one another. In particular, behaviours frequently evolve to increase the efficacy of an animal's physical traits, such that its overall protection from predators is improved. For example, camouflaged moths often orient themselves on trees to maximize crypsis (Webster et al. 2009; Kang et al. 2012), while noxious newts signal their unpalatability to on-looking predators through a rigid defensive posture that displays their bright ventral colouration (Johnson \& Brodie 1975). Similarly, mimetic animals may employ behaviours to increase their resemblance to dangerous or noxious models. Spectacular examples of this include hoverflies that hold up and wave their dark front legs in front of their head to mimic the conspicuous antennae of wasps (Waldbauer 1970; Penney et al. 2014), and mimetic butterflies that fly slowly and in a straight line to mimic the nonchalant flight behaviour of their models (Srygley 1999, 2004). Clearly behavioural mimicry can play a key role in making the discrimination task of the predator more difficult, thereby conferring greater protection to mimetic prey.

While naturalists have long recognized that many lepidopteran caterpillars possess conspicuous eye-like markings (“eyespots"), many have also noted that when threatened these caterpillars adopt a defensive posture that seems to increase their resemblance to a snake (e.g., Bates 1862; Weismann 1882; Poulton 1890; Cott 1940). Specifically, when confronted with a perceived threat, many of these caterpillars inflate 
their anterior body segments and/or pull their head into their body telescopically such that the anterior segments are similarly inflated (Bates 1862; Weismann 1882; Poulton 1890). Fear responses to caterpillars bearing eyespots and displaying their defensive posture have been anecdotally described in taxa ranging from birds to primates (see descriptions from (Poulton 1890; Cott 1940).

Recent work has demonstrated that a fixed defensive posture in artificial caterpillars is capable of conferring protection from insect-eating birds in the field (Hossie \& Sherratt 2013). Widening the anterior body segments may function to deter attack for a number of reasons including emphasizing the eyespots, bluffing the potential predator by appearing larger, and enhancing the caterpillar's overall resemblance to snakes. The latter explanation in particular remains speculative without an objective measure of mimetic fidelity in both rest and defensive postures. More fundamentally, despite widespread acceptance that caterpillars with eyespots mimic snakes, we are not aware of any attempt to quantify this resemblance.

Although subjective, inflation of the anterior segments appears to increase the 'head-like' appearance of the anterior body segments (particularly in those species with eyespots, TJH and TNS pers. obs.). This is certainly the case for a range of temperate and tropical caterpillars that we have had the opportunity to observe directly (incl., temperate: Papilio canadensis, P. cresphontes; tropical: Eumorpha phorbas, Hemeroplanes triptolemus, Therinia transversaria, Xylophanes cthulhu). The defensive posture often creates a wider or more triangular-shaped head which seems viper-like on cursory examination, supporting the occasional suggestion that at least some caterpillars with eyespots mimic vipers specifically (Bates 1862; Valkonen et al. 2011a). Yet, the snake 
families Boidae, Colubridae and Viperidae all contain several species that are important predators of insect-eating birds, and thus snakes from any of these groups pose an imminent mortality threat to insectivorous birds. As such there seems to be little reason to suspect that these caterpillars mimic vipers specifically, as opposed to snake mimicry more generally. Indeed, Janzen et al (2010) suggested that eyespots function because birds possess a generalized aversion to the eyes of threatening vertebrates in general (i.e., including but not limited to snakes). Venomous snakes may however pose a unique threat in that non-predatory attacks can also result in mortality, and even those snakes too small to consume an insect-eating bird pose considerable risk. Further, large birds and other non-avian insectivores may not be similarly deterred from attacking what appears to be a small snake unless it appears to be venomous. Valkonen et al. (2011) showed that a viper-like head shape reduced predation by raptors on model snakes (compared to models with a "colubrid-like" head shape), and in combination with our work (Hossie \& Sherratt 2013) it seems that birds are generally more cautious when dealing with snake-like prey possessing wide "heads". Thus, it remains possible that the defensive posture of at least some caterpillars increases their resemblance to Viperidae snakes specifically. Clearly however, the contention that caterpillars with eyespots resemble a Viperidae snake more so than a Colubridae snake requires an objective measure of mimetic fidelity.

Here we have employed a landmark-based shape analysis (i.e., geometric morphometrics, see Zelditch et al. 2004) to quantify the head shape and position of eyes in snakes, to the "head" shape and position of eyespots in putative snake-mimic caterpillars. Specifically, we have attempted to answer two related questions: 1) Does the defensive posture of caterpillars with eyespots increase their resemblance to snakes?, and 
2) Do these caterpillars resemble Viperidae snakes more so than Colubridae snakes when they adopt their defensive posture?

\subsection{Methods}

Specimens

We photographed 10 Papilio canadensis (Papilionidae) caterpillars which were hand-reared from eggs laid by wild-caught females collected in Kemptville, Ontario Canada during the summer months of 2011. All Papilio caterpillars came from 2 females and were photographed during the final (5th) instar in both the rest and defensive posture from both the lateral and dorsal view. Photos of the putative snake mimic Therina transversaria (Saturniidae) were taken by TJH of a caterpillar collected by parataxonomists as part of an ongoing inventory project in the Área de Concervación Guanacaste (ACG), Costa Rica (Janzen et al. 2005; Janzen \& Hallwachs 2011).

Remaining photos were taken from Janzen and Hallwachs (2014), an online database that catalogues the results of the aforementioned inventory project in the ACG. We searched this database for photos of caterpillars with eyespots that clearly showed the caterpillar from a dorsal or lateral view. Photos were deemed appropriate for this work only if the view of the caterpillar was not rotated, that is, the camera shot was directly over the caterpillars body (dorsal view) or directly revealed the lateral profile of the caterpillar (lateral view). Although this process limited the number of species that could be included in our analysis it was necessary because only these photos could be used to accurately quantify the position of the features of interest (i.e., eyespots, and "head shape") and compare them to their putative models. Further, only those species from which we were 
able to collect a photo of the caterpillar in both the rest and the defensive posture were included in our analysis. This was important because it would be difficult otherwise to determine whether the difference in morphology between "rest" and "defensive" photos truly resulted from a change in body posture, and was not simply an artifact of among species variation. Overall, we were able to obtain rest and defensive posture photos for 22 lepidopteran species in total, however only 14 of these species had suitable photos in both lateral and dorsal views. To create shape variables (see below) we used 18 species in the dorsal view and 18 species in lateral view; 4 species in each analysis differed between dorsal and lateral views (see Table S4-1). The number of caterpillar photographs available (i.e., photos of different individuals) for each species varied from 1-5 (see Table S4-1), and in cases where multiple images were available we used the average morphology from these photos in our analyses (see below).

Preserved snake specimens from the collections at the Canadian Museum of Nature, Ottawa were photographed from the dorsal and lateral orientations. It seems unlikely that a given caterpillar with eyespots mimics a specific species, but is instead a more general mimic of animals such as snakes that pose an imminent threat to insecteating birds (Janzen et al. 2010). We therefore collected photos of snake specimens from a range of taxonomic groups that vary in morphology. This approach is in line with our goal which was to characterize the morphospace occupied by snakes in general, as well as colubrid- and viperid-specific morphospaces, such that we could determine the extent of overlap with the caterpillars when adopting their resting and defensive postures. This approach is also conservative in that it minimizes the possibility that failing to select a particular snake species would affect our final conclusions. While we were somewhat 
limited by availability we were able to photograph 82 preserved snakes, representing 36 species from 19 genera, within six subfamilies (Table S4-2). Sixteen of these species were from Colubridae, 19 from Viperidae, and one species was from Boidae (Table S42). Dorsal photos required the eye not to be damaged or sunken into the orbit, and lateral photos were only used if the specimen's mouth was closed or could be closed without distorting the specimen. After close inspection of the snake photos for quality control two species were not included in the dorsal analysis and three species were not included in the lateral analysis because the photos were unsuitable (see Table S4-2). Overall, the number of photos (of different individuals) for each species of snake ranged from 1-6; where multiple photos were available the average morphology used in the analyses (see below).

\section{Measurements}

Landmarks were digitized on all photos using tpsDig2 software (Rohlf 2006) following the landmark placements detailed in Table 4-1 (see also Figure 4-1). We contend that although the landmarks are not placed on anatomically analogous locations between snakes and caterpillars they reflect apparently analogous features in both taxa as seen by a potential predator such as an insect-eating bird (see also Benitez-Vieyra et al. 2009 for a similar approach). This landmark data was read into CoordGen7a (Sheets 2011a) where we conducted a Procrustes transformation to eliminate effects of size, rotation, and translation on shape (Zelditch et al. 2004). In cases where more than one specimen was available within a species (and also within posture for caterpillars) a consensus mean (i.e., a single average set of landmark coordinates for each species) was generated using the reference specification function in CoordGen7a. This process 
minimized inaccurate assignment of landmark positions within species. We then conducted two principal component analyses (PCAs) using PCAGen7a (Sheets 2011b) to describe and quantify the variation in shape for both dorsal and lateral views. In each PCA we included landmark data from snakes and from caterpillars in both resting and defensive postures. PCA was selected over other methods, such as canonical variates analysis, because it does not require placing the specimens into pre-defined groups and thereby objectively determines which shape features best describe shape variation in the data set. Principal components (PCs) which described more than $10 \%$ of the variation in shape and laid to the left of the "elbow" in the scree plot were then used to describe the morphospace occupied by caterpillars in each posture and snakes, respectively. 
Table 4-1: The position of landmarks for geometric morphometric comparison of 'eyespots' and body shape of caterpillars with eyespots and the corresponding landmark positions on snakes.

\section{Lateral View}

\begin{tabular}{cll}
\hline Landmark & Caterpillar & Snake \\
\hline $\mathbf{1}$ & Center of eyespot & Center of eye \\
\hline $\mathbf{2}$ & Top of eyespot & Top of eye \\
\hline $\mathbf{3}$ & Bottom of eyespot & Bottom of eye \\
\hline $\mathbf{4}$ & Anterior extent of eyespot & Anterior extent of eye \\
\hline $\mathbf{6}$ & Posterior extent of eyespot & Posterior extent of eye \\
\hline $\mathbf{7}$ & ${\text { Behind } 1^{\text {st }} \text { segment behind head }}^{\text {Top of body above center of eyespot }}$ & Tip of snake nose \\
\hline $\mathbf{8}$ & Bottom of body below center of eyespot & Top of head above center of eye \\
\hline $\mathbf{9}$ & Top of body after the $6^{\text {th }}$ body segment & Bottom of head below center of eye \\
\hline $\mathbf{1 0}$ & Directly below LM 9 & Top of body, where neck meets the head \\
\hline
\end{tabular}

Dorsal View

\begin{tabular}{cll}
\hline Landmark & Caterpillar & Snake \\
\hline $\mathbf{1}$ & ${\text { Behind }{ }^{\text {st }} \text { segment behind head }}^{\text {Anterior extent of left eyespot }}$ & Tip of snake nose \\
\hline $\mathbf{2}$ & Anterior extent of left eye \\
\hline $\mathbf{3}$ & Center of left eyespot & Center of left eye \\
\hline $\mathbf{5}$ & $\begin{array}{l}\text { Left eyespot, closest point toward the } \\
\text { midline }\end{array}$ & $\begin{array}{l}\text { Left eye, closest point towards center of } \\
\text { head }\end{array}$ \\
\hline $\mathbf{6}$ & Left eyespot, furthest point to the left & Left eyespot, furthest point to the left \\
\hline $\mathbf{7}$ & Posterior extent of left eyespot & Posterior extent of left eye \\
\hline $\mathbf{8}$ & Anterior extent of right eyespot & Anterior extent of right eye \\
\hline $\mathbf{9}$ & Center of right eyespot & Renter of right eye \\
\hline $\mathbf{1 0}$ & midline & $\begin{array}{l}\text { Right eye, closest point towards center of } \\
\text { head }\end{array}$ \\
\hline $\mathbf{1 1}$ & Posterior extent port of right eyespot & Right eyespot, furthest point to the left \\
\hline $\mathbf{1 2}$ & Widest point on the left side of body & Posterior extent of right eye \\
\hline $\mathbf{1 3}$ & Widest point on the right side of body & Widest point on the left side of head \\
\hline $\mathbf{1 4}$ & After $6^{\text {th }}$ body segment, left extent & Widest point on the right side of head \\
\hline $\mathbf{1 5}$ & After $6^{\text {th }}$ body segment, right extent & Right extent of body after the head \\
\hline & &
\end{tabular}




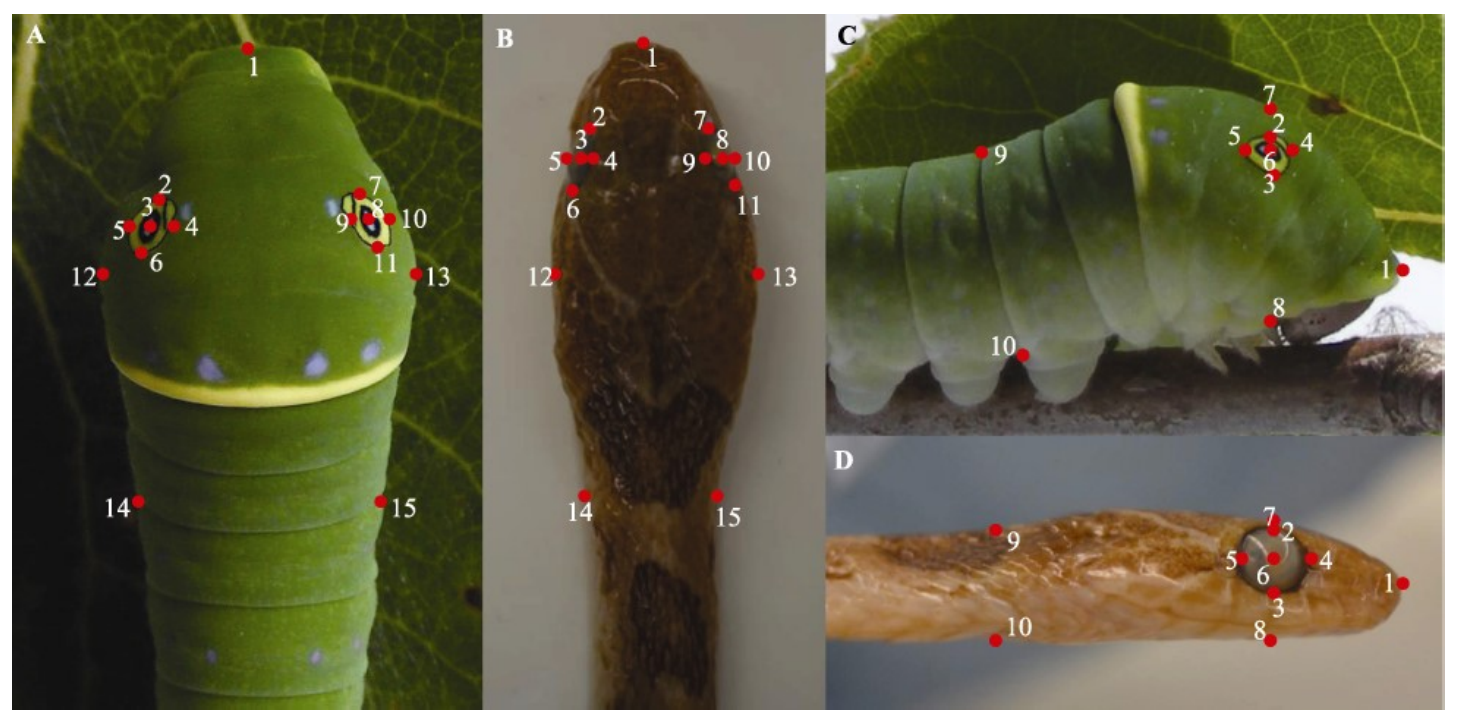

Figure 4-1: Landmark placements for caterpillars and snakes in their dorsal and lateral views. A) Papilio canandensis (Papilionidae) caterpillar in defensive posture, dorsal view; B) Leptodeira annulata (Colubridae) snake, dorsal view; C) P. canadensis caterpillar defensive posture, lateral view; D) $L$. annulata, lateral view. 
Analyses

We determined which of the significant PCs described a change in apparent shape between caterpillars in the resting and defensive posture using matches-pairs t-tests (i.e., paired by species). As we were interested in how a change in posture affected resemblance to snakes, only those PCs that showed significant difference in shape between postures were included in subsequent Linear Discriminant Analyses (LDAs) described below. First, we examined whether caterpillars in the defensive posture appear more snake-like than caterpillars in the resting posture by developing a LDA model to discriminate between caterpillars and snakes. To develop the model we included data from caterpillars in the resting posture and all the snake species sampled, but excluded data from caterpillars in their defensive posture. This model was then used to classify caterpillars in the defensive posture as either caterpillars or snakes (using a posterior probability threshold of $>0.5$ ). Posterior probabilities for the classification of resting and defensive posture caterpillars were extracted and we used a matched-pairs t-test to examine whether the probability of classification as a snake changed with posture. Next, we created a second LDA model to determine whether caterpillars in the defensive posture look more like vipers than colubrids. Specifically, a model was developed to discriminate among caterpillars, Colubridae snakes, and Viperidae snakes using data from caterpillars in their resting posture and all colubrid and viperid snake species sampled, but excluding data from caterpillars in their defensive posture. The resulting LDA model was then used to classify caterpillars when in their defensive posture (i.e., as a caterpillar, Colubridae snake, or Viperidae snake). Using the posterior probabilities of classification from the LDA model we tested whether the probability of classification of 
the caterpillars as a caterpillar, Colubridae snake, or Viperidae snake changed between postures with dependent t-tests. A final LDA model that included all significant PCs (i.e., including those that did not differ significantly with posture) was developed in the same way to determine the relative importance of each PC in discriminating among groups when all information is available. Differences between snake families (Colubridae and Viperidae) in dorsal and lateral PCs were tested using Welch's t-tests. All LDAs and ttests were conducted using R statistical software (R Development Core Team 2014).

\subsection{Results}

In quantifying the variation in shape when considering caterpillars in resting and defensive posture, as well as snakes from Colubridae and Viperidae (and one Boidae species), our PCA revealed that two PCs explained the majority of the variance in shape for the dorsal view ( $\%$ variance explained: $\mathrm{PC} 1=63.6 \%, \mathrm{PC} 2=19.6 \%$ ), and similarly two PCs explained the majority of the variance in the lateral view (\% variance explained: $\mathrm{PC} 1=37.7 \%, \mathrm{PC} 2=33.4 \%$ ). A greater dorsal PC1 value indicates a more anterior position for the widest points on the "head", a slight posterior shift of the "eyes", and an anterior lengthening of the "head" (Figure 4-2). In contrast an increase in dorsal PC2 represents a widening of the "head" with concurrent movement of the "eyes" away from the midline, and the anterior point moving in towards the body shortening the "head" (Figure 4-2). As the lateral PC1 value increases the body becomes dorso-ventrally thicker, the "eyes" move dorsally, and the anterior point of the body comes in toward the body (Figure 4-2). Finally, an increase in lateral PC2 indicates a dorso-ventral thickening, slight enlargement of the "eye", and a lengthening of the anterior point of the head 
(Figure 4-2). The morphospace occupied by each group in the dorsal and lateral view is depicted in Figure 4-3. 


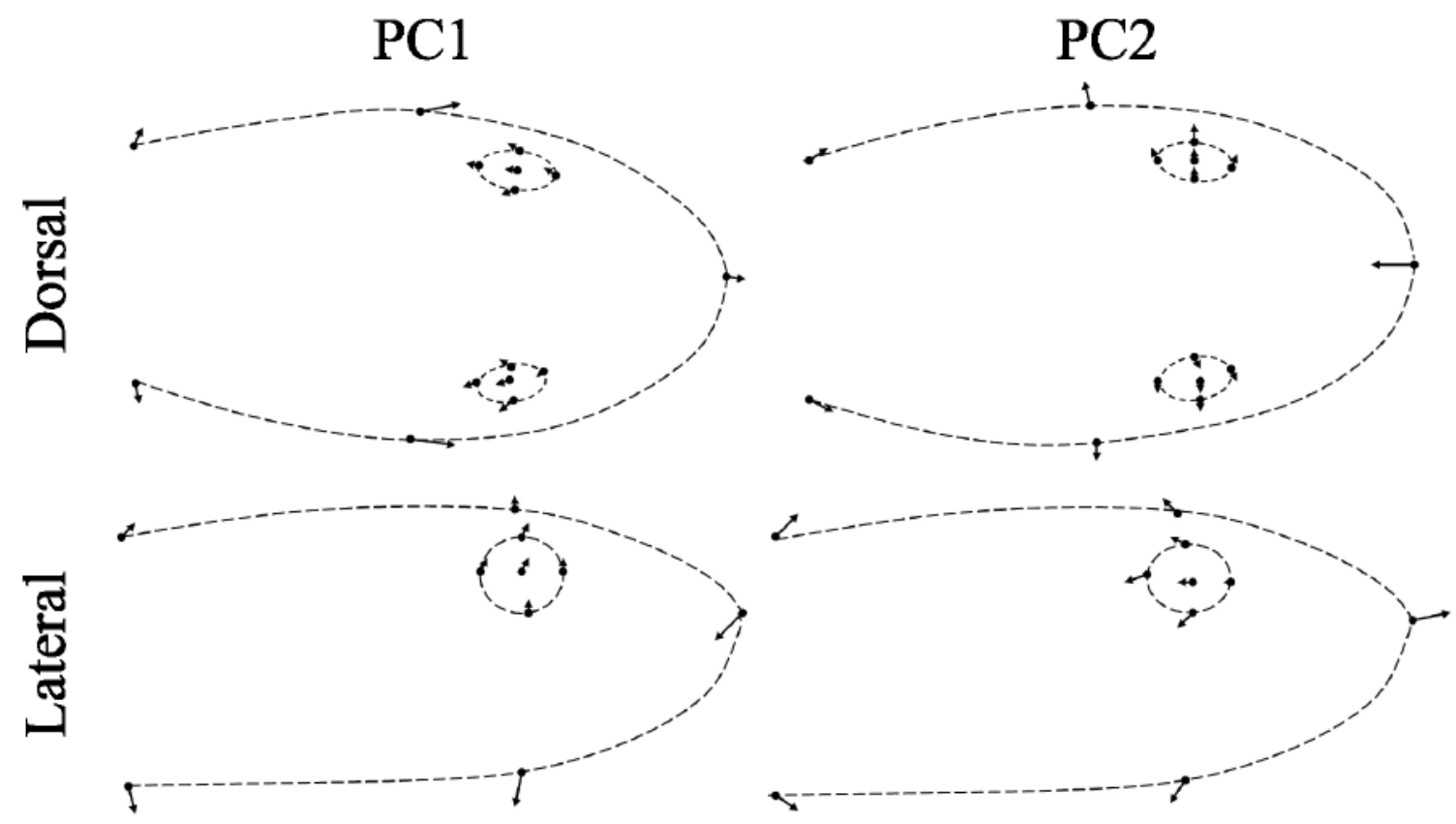

Figure 4-2: Vector plots illustrating the shape change described by principal components (PCs) 1 and 2 for both the dorsal and lateral view. Vectors point in the direction of an increasing PC value. 

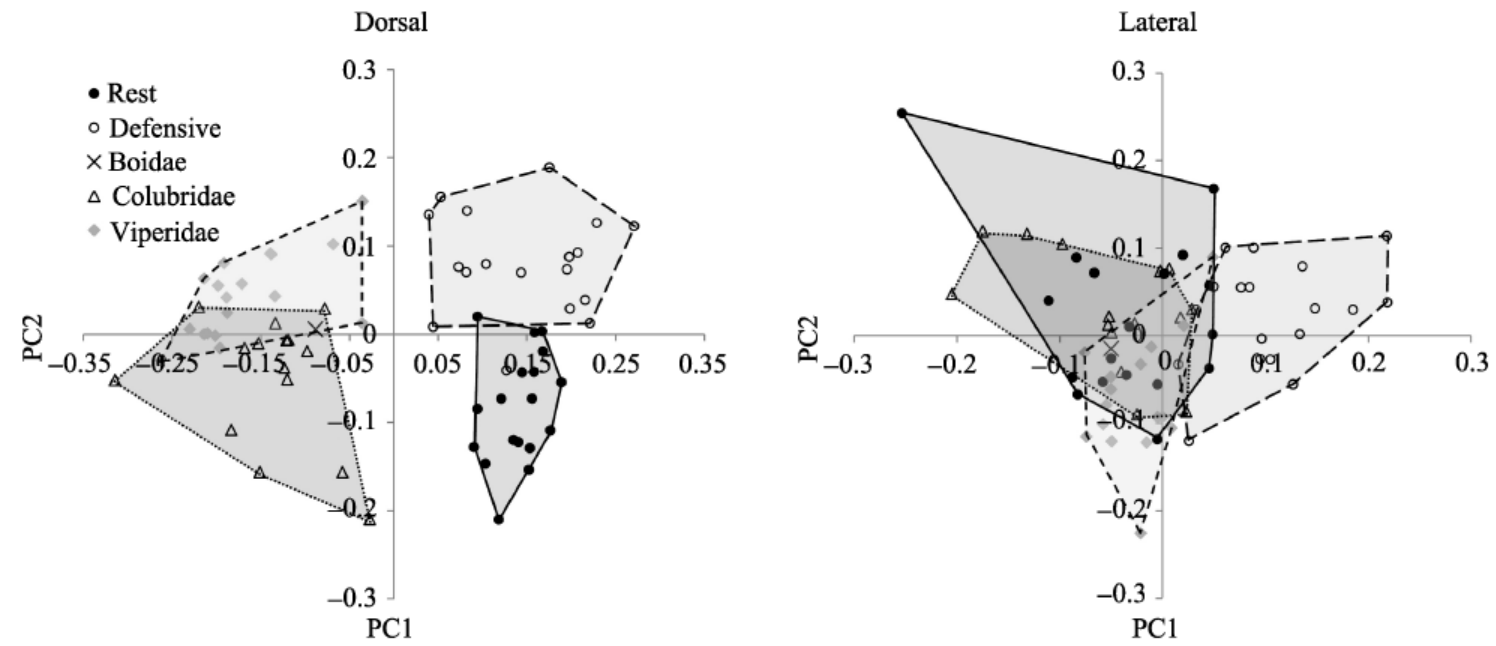

Figure 4-3: The morphospace occupied by caterpillars with eyespots in their resting and defensive postures as compared to Colubridae and Viperidae snakes. Only one Boidae snake was measured and its location is demarked with an X. 
Only two PCs described shape variation that differed significantly between the caterpillars in the resting and defensive posture: dorsal PC2 and lateral PC1 (Table 2, Figure 4-3). Yet only dorsal PC2, and not lateral PC1, differed significantly between Colubridae and Viperidae snakes (dorsal PC2: $t_{23.28}=-4.215, P=0.0003$, lateral PC1: $\left.t_{19.89}=-1.414, P=0.17\right)$. The first LDA was only able to correctly identify $50 \%(7 / 14)$ of the rest caterpillars and only misclassified 13\% (4/32) of snakes as caterpillars. When caterpillars in their defensive posture were classified by this model, an equivalent $50 \%$ of the caterpillars were identified as snakes (i.e., had posterior probability of $>0.5$, Table S44). Caterpillars from three species shifted from being classified as a caterpillar in the rest posture to a snake when in the defensive posture, and similarly three species classified as snakes in the rest posture were classified as caterpillars in the defensive posture (Table S4-4). A dependent t-test did not indicate a significant change in the posterior probability of classification as a snake when considering all species examined $(\mathrm{t}=-0.41, \mathrm{df}=13, P=$ 0.69), although some species clearly became more snake-like (Table S4-4). 
Table 4-2: Results from matched-pairs t-tests comparing the shape of caterpillars in their rest and defensive postures for each of four shape variables (i.e. principal components).

\begin{tabular}{|c|c|c|c|c|c|c|c|}
\hline \multicolumn{8}{|l|}{ Principal } \\
\hline Component & Posture & Mean & SD & $\mathbf{n}$ & $\mathbf{t}$ & df & p-value \\
\hline \multirow[t]{2}{*}{ Dorsal PC1 } & Rest & 0.14 & 0.03 & 18 & -0.04 & 17 & 0.63 \\
\hline & Defensive & 0.148 & 0.0733 & 18 & & & \\
\hline \multirow[t]{2}{*}{ Dorsal PC2 } & Rest & -0.082 & 0.0626 & 18 & -9.734 & 17 & $<0.0001$ \\
\hline & Defensive & 0.082 & 0.0581 & 18 & & & \\
\hline \multirow[t]{2}{*}{ Lateral PC1 } & Rest & -0.037 & 0.0751 & 18 & -6.356 & 17 & $<0.0001$ \\
\hline & Defensive & 0.105 & 0.0606 & 18 & & & \\
\hline \multirow[t]{2}{*}{ Lateral PC2 } & Rest & 0.023 & 0.0927 & 18 & -0.049 & 17 & 0.96 \\
\hline & Defensive & 0.024 & 0.0608 & 18 & & & \\
\hline
\end{tabular}


In the second LDA, $57 \%(8 / 14)$ of rest caterpillars were correctly identified as caterpillars ( 6 were classified as Colubridae snakes), whereas $71 \%(22 / 31)$ of snakes were correctly assigned to family. Three colubrid snakes were misclassified as vipers, but only two vipers as a colubrid. Four snakes were misclassified as caterpillars (three Colubridae and one Viperidae). This LDA model correctly classified 57\% (8/14) of the defensive posture caterpillars as caterpillars, the remaining $43 \%$ (6/14) were misclassified as vipers. Of the caterpillars misclassified as vipers, three had been classified as caterpillars in the resting posture and three had been classified as colubrid snakes (Table S4-4). Dependent t-tests revealed that upon adopting the defensive posture caterpillars generally became significantly less caterpillar-like $(t=8.09$, df $=13, P<0.001)$, significantly more viper-like $(t=-3.34, \mathrm{df}=13, P=0.005)$, but not significantly more colubrid-like $(t=-0.67, \mathrm{df}=13, P=0.52)$.

Data inspection revealed that a shift to the defensive posture actually decreased mimetic fidelity in terms of the shape change described by lateral PC1 (Figure 4-3). In addition, only dorsal PC2, but not lateral PC1, differed between colubrid and viperid snakes. Taken together this indicated that mimicry might be operating from the dorsal view only. Thus, we conducted an additional LDA using only dorsal PC2 to examine classification rates under the assumption that predators primarily use this information. Similar to the previous analysis (i.e., LDA 2) we found that $8 / 14$ resting caterpillars were classified as caterpillars (4/14 as colubrids, 2/14 as vipers), but when the model was applied to the defensive posture all 14 species were classified as snakes $(1 / 14$ as a colubrid, 13/14 as vipers, Figure 4-4). Importantly, dependent t-tests confirmed that caterpillars were less "caterpillar-like" in the defensive posture $(t=9.67, \mathrm{df}=13, P$ 
$<0.001)$, but also that they were less colubrid-like $(t=6.90$, df $=13, P<0.001)$ and more viper-like $(t=-10.63, \mathrm{df}=13, P<0.001)$. 


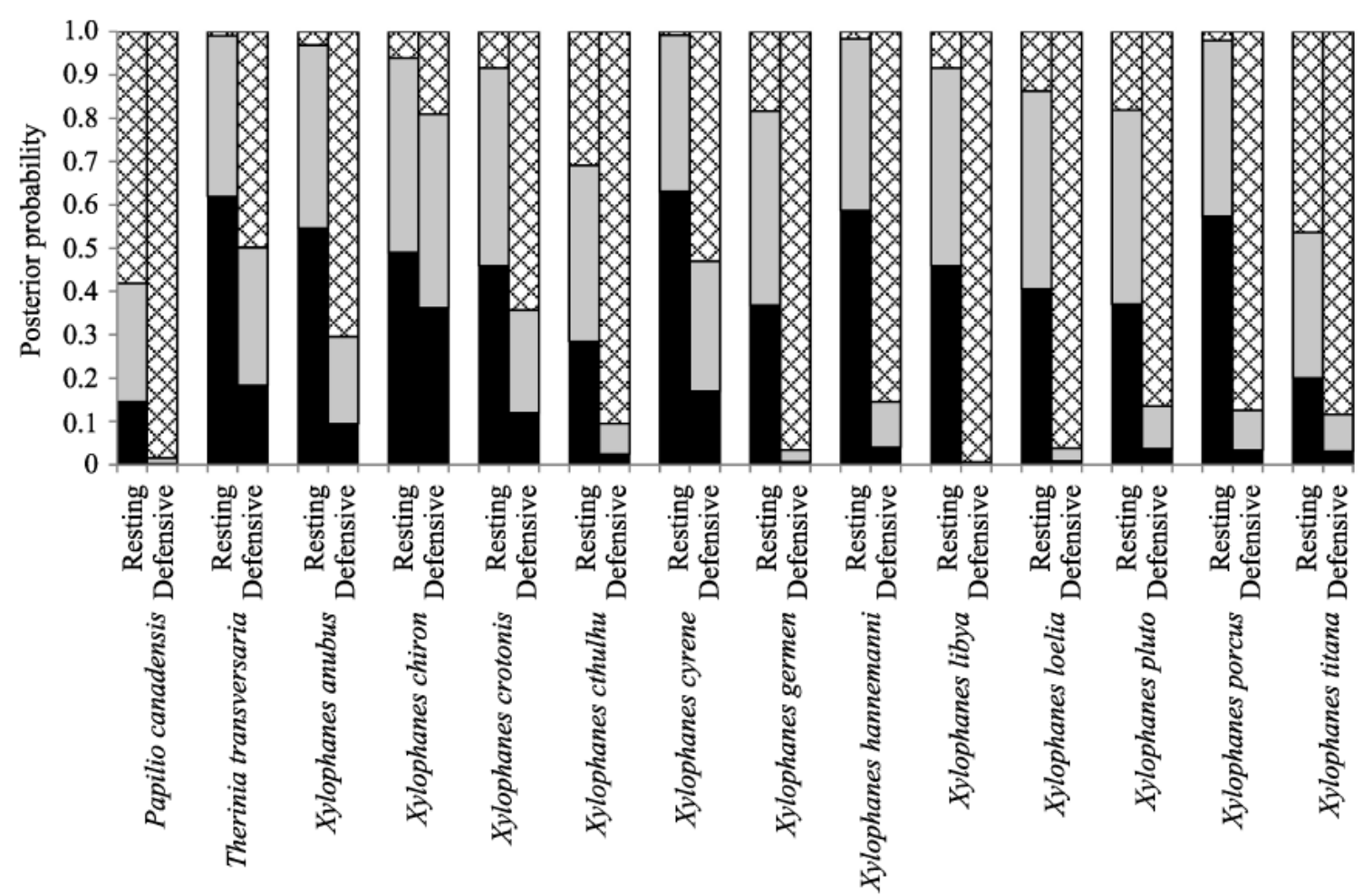

Figure 4-4: Posterior probabilities for the classification of caterpillars from 14 species as a caterpillar (black), colubrid snake (grey), or viperid snake (hatched) when in their resting and defensive posture. Posterior probabilities shown here were extracted from a linear discriminant analysis using only dorsal features that changed with posture (i.e., dorsal PC2 only). 
Finally, the overall LDA model which included all four PCs (i.e. PC1 and PC2 from both dorsal and lateral views) had the most accurate assignment rates. All rest caterpillars were correctly assigned as caterpillars (i.e., in both resting and defensive postures), two Colubridae snakes were misclassified as vipers, and one viper was misclassified as a Colubridae snake. The standardized coefficients of the linear discriminants for all LDA models are provided in the electronic supplementary material (Table S4-3).

\subsection{Discussion}

We quantified the head morphology of preserved snakes as well as the shape of the anterior body segments (i.e., the apparent head) of 14 caterpillars with eyespots in their resting and defensive postures to test for evidence of behavioural mimicry. Specifically, we tested whether the defensive posture of these caterpillars increases their resemblance to snakes in general, or if it increases their resemblance to vipers. Our first discriminant analysis was developed to distinguish between resting posture caterpillars and snakes, but only assigned $50 \%$ of the resting caterpillars correctly. Applying this model to the same caterpillars when in their defensive posture revealed that the change in posture did not significantly increase the probability of classification as a snake. We therefore did not find sufficient evidence to indicate that the defensive posture increases (or reduces) the resemblance of these caterpillars to snakes in general.

The second discriminant analysis, developed to distinguish among caterpillars at rest, Colubridae snakes, and Viperidae snakes, revealed that caterpillars in their defensive posture were more viper-like compared to when in their resting posture. Also, no 
caterpillar in the defensive posture was misclassified as a colubrid snake, but $43 \%$ were misclassified as vipers. As the lateral view reduced mimetic fidelity upon adopting the defensive posture (see Figure 4-3), and did not differ between colubrid and viperid snakes, an additional analysis assessed how caterpillars would be classified if only the key dorsal features (i.e., dorsal PC2) were used. This analysis revealed that the classification of caterpillars shifted significantly towards a more viper-like appearance and away from that of a resting caterpillar or colubrid snake (Figure 4-4). All 14 caterpillars in the defensive posture were classified as snakes (13/14 as vipers, $1 / 14$ as a colubrid). Our analyses thus provide statistical evidence for behavioural mimicry in all species examined (including species from Papilionidae, Sphingidae, and Saturniidae), and reveal that the shift in posture helps these caterpillars mimic dorsal, but not lateral, features of venomous snakes. Of course, just because the caterpillars happen to look more viper like does not prove that they are adopting this posture in order to improve their mimetic fidelity - the enhanced similarity could be entirely incidental. However, our analysis is consistent with this theory, as well as repeated anecdotal observations that the caterpillars become more snake like when they adopt their defensive postures.

Our final LDA which included all four shape variables (PCs) did not misclassify any caterpillars as snakes, even when the caterpillars adopted the defensive posture. This indicates that it should be possible for a predator to discriminate between snakes and each of the caterpillars examined here based solely on the features we quantified. Critically, perfect mimicry is not required to yield a selective advantage over non-mimics (Edmunds 2000; Penney et al. 2012); even small adjustments in mimetic fidelity can result in large differences in the degree of protection, particularly when the model is highly noxious 
(Duncan \& Sheppard 1965; Dittrich et al. 1993; Sherratt 2002). It is therefore likely that the apparent morphological changes resulting from a shift in posture, as quantified here, translate into a significant protective effect. Indeed, we have recently shown such an effect in the field using model caterpillars designed to represent the resting and defensive postures (Hossie \& Sherratt 2013). Predators also face a speed-accuracy trade-off which imposes limits to the amount of information that can be acquired and instead favours rapid decisions based on conspicuous features (Chittka \& Osorio 2007; Abbott \& Sherratt 2013). Insect-eating birds likely adopt a "safety first" strategy in order to balance the benefit of avoiding an imminent mortality threat which far outweighs the cost of losing a meal. Such a strategy would enable caterpillars to gain protection from even relatively poor levels of mimetic fidelity (Ruxton 2005; Bura 2010; Olofsson et al. 2012). Finally, we note that by adopting a more viper-like "head" shape the caterpillars resemble a snake that is less frequently confused with caterpillars (as judged by our analyses). Therefore increasing resemblance to vipers may simply aid in concealing the caterpillar's true identity, as opposed to indicating that viper-specific resemblance has arisen because vipers pose a greater threat to attackers.

Although the apparent head shape of caterpillars in the defensive posture increases the caterpillar's resemblance to Viperidae snakes when viewed dorsally, adopting the defensive posture did not increase resemblance to either snake family (or to snakes in general) from the lateral view. Features visible from above, such as apparent head width, may be under stronger selection to resemble snakes because insect-eating birds often view caterpillars from above, or because dorsal features more reliably distinguish colubrid and viperid snakes. Constraints relating to caterpillar anatomy may 
also help explain the lack of similarity of the defensive posture when viewed laterally. For example, inflating the anterior body segments to achieve the goal of widening the apparent head might simultaneously deepen the "head" due to the caterpillar's soft exoskeleton. This explanation is consistent with our morphometric analysis which illustrated that a change from rest to defensive posture resulted in a wider but shorter apparent head dorsally (dorsal PC2), and deeper but shorter apparent head laterally (lateral PC1). There is however substantial overlap between resting caterpillars and snakes in the lateral morphospace (Figure 4-3), indicating that resting caterpillars may effectively mimic snakes while resting so long as they are viewed laterally. Briefly, we highlight that our method of quantifying mimetic fidelity is distinct from other approaches (e.g., Taylor et al. 2013), but such landmark-based approaches are particularly well suited for examining changes in similarity due to behaviour (i.e., behavioural mimicry) and offer a promising tool for future research. Landmark-based methods are useful when analogous features (e.g., eyespots vs. real eyes) are easily denoted among models and mimics (even if such features are not homologous), whereas methods akin to Taylor et al. (2013) are likely to be superior in cases where colour patterns are not immediately analogous (e.g., yellow and black bands on mimetic flies and their hymenopteran models).

Under what conditions should we expect behavioural mimicry to evolve? Does it arise in relatively good mimics reflecting the comparatively strong selection for mimetic perfection or in poor mimics as a means of compensation? Initial evidence in hoverflies suggests that behavioural mimics also tend to be relatively good morphological mimics (Penney et al. 2014). Anecdotally, this also seems to be true for caterpillars with eyespots 
as the most complete case of behavioural mimicry of which we are aware occurs in caterpillars from the Hemeroplanes genus (Sphingidae). In the defensive posture these species show an uncanny resemblance to snakes, and further extend the deception by reaching out from the tree in a display that convincingly resembles an arboreal viper outstretched from a branch (TJH, personal observation; see photos in Janzen \& Hallwachs 2014). Further, we expect behavioural mimicry in caterpillars with eyespots to be more pronounced in large species for a number of reasons. First, large species are more profitable prey items making predators more inclined to search them out and less easily deterred from attack upon encounter (see also Penney et al. 2012). Second, large prey are more conspicuous and may therefore be under stronger selection to evolve defences that offer protection post-encounter. Third, behavioural mimicry may be more convincing (and therefore effective) when the caterpillar falls closer to the size range of real snakes. More generally, larger "items" may simply be more likely to pose a significant threat, thereby favoring startle or mimicry defences more strongly in relatively large prey. Finally, large caterpillars may be more readily attacked by non-avian predators that utilize a different set of cues to identify snakes and thereby require more complete mimicry to deceive a wider range of predators.

Although our results provide an important step towards understanding mimicry in this system by quantifying mimetic fidelity of caterpillars with eyespots to their putative models, interpretation of our results should be tempered by the following considerations. First, our analyses only considered snakes as possible models, whereas lizards and other dangerous vertebrates may serve to generate or reinforce the aversion to attacking eyespot caterpillars. We also acknowledge the possibility that the specific snake species 
used in our analysis may have influenced our results. Specifically, the head morphology of some colubrid snakes not included here may be more similar to that of the "viper-like" head shape described by our analyses. However, we attempted to select species from a range of taxonomic lineages with diverse head morphologies, and thus our results should be robust to the addition or removal of a given species. Unfortunately, we were not able to measure enough Boidae species to compare mimetic fidelity to these snakes directly, although these snakes likely pose an important risk to birds and may represent an important model. Due to a lack of photos we did not characterize the head-on view of caterpillars which may also play an important role in the mimetic resemblance to snakes, particularly for those caterpillars that hide in leaf shelters. Similarly, a lack of available photos limited our investigation to 3 families within Lepidoptera, the majority of which came from one genus (Xylophanes). Finally, we note that there are several caterpillars that lack or have poorly developed eyespots which adopt defensive postures similar to those described here, and a mimetic function is suspected in at least some of these species (e.g., Eumorpha phorbas, Papilio cresphontes).

The evolution of eyespots and coincident behavioural mimicry could have important evolutionary consequences within Lepidoptera, such as influencing the rate of speciation. There are several examples of species-rich genera within Lepidoptera which have independently evolved eyespots, and the associated behavioural mimicry discussed here, including two that we examined: Papilio (Papilionidae) and Xylophanes (Sphingidae). Whereas caterpillars that employ crypsis rely on host-specific colour matching for effective concealment, and aposematic caterpillars rely on secondary metabolites from their host plant to gain toxicity, caterpillars with eyespots and deceptive 
behaviours are diminished in their reliance on a specific host plant for protection from predators possibly facilitating radiation. Ongoing research will examine this possibility that species with caterpillars employing deceptive anti-predator defences (e.g., snake or bird-dropping mimics) radiate more rapidly. The augmented rates of speciation observed in orchids (Orchidaceae) have been similarly explained by the evolution of deception, though within a different context (Cozzolino \& Widmer 2005; Papadopulos et al. 2013). Doubtless, the numerous examples of caterpillars which appear to mimic snakes or other threatening vertebrates indicates that deception has played an important role within Lepidoptera, and has likely influenced each species' behaviour or life history in ways yet to be discovered.

\subsection{Acknowledgements}

We thank Carita Lindstedt-Kareksela and Mikael Mokkonen for their invitation to participate in this special edition on deception. We thank Michèle Steigerwald and the Canadian Museum of Nature, Ottawa for access to collections. Chris Hassall and two anonymous referees provided useful comments on an earlier version of this manuscript. We also thank Dan Janzen and Winnie Hallwachs for the opportunity to observe the behaviour of several tropical caterpillars in the field. Funding for this research was provided by a Discovery Grant (NSERC) awarded to TNS. 


\title{
5 Chapter: An eyespot that "blinks": an open and shut case of eye mimicry in Eumorpha caterpillars (Lepidoptera: Sphingidae)
}

\author{
Thomas J. Hossie ${ }^{1 *}$, Thomas N. Sherratt ${ }^{1}$, Daniel Janzen ${ }^{2}$ and Winnie Hallwachs ${ }^{2}$ \\ ${ }^{1}$ Department of Biology, Carleton University, Ottawa, ON, Canada \\ ${ }^{2}$ Department of Biology, University of Pennsylvania, Philadelphia, PA, USA \\ *Corresponding author
}

Published in Journal of Natural History (2013) 47: 2915-2926

Reproduced in whole in accordance with Taylor and Francis policy. 


\section{$5.1 \quad$ Abstract}

We describe the final instars of two tropical Sphingidae caterpillars - Eumorpha phorbas and Eumorpha labruscae - from Área de Conservación Guanacaste, northwestern Costa Rica, whose anal horn has become a posterior eyespot structure capable of rapid palpitation. When approached or harassed, the caterpillars palpitate this eyespot and produce the effect of a blinking vertebrate eye. We propose that this "blinking" is an extension of eye-mimicry or at least draws attention to the eyespot, functioning to startle and/or intimidate would-be predators. Since snakes lack eyelids and do not blink, this suggests that the blinking eye represents a more generalized eye, or a mammalian eye. Eyespot "blinking" is probably controlled by the same musculature used to wave the anal horn in earlier instars. The extent to which this eyespot is perceived as a blinking eye, and the degree of protection from the caterpillars' suite of potential predators, remains to be discovered. 


\subsection{Introduction}

Eyespots are circular or elliptical markings that occur on the bodies of many animals. To varying degrees, these markings resemble either a single or pair of vertebrate eyes (reviewed by Stevens 2005). The function typically ascribed to eyespots is protection from, or during, a predatory attack (Edmunds 1974; Ruxton et al. 2004a; Janzen et al. 2010), although other (or additional) processes can also generate and maintain similar markings including sexual selection when the eyespots are on adults (e.g., Breuker and Brakefield 2002; Oliver et al. 2009). The eyespots of many animals are kept concealed, then revealed suddenly when the prey is approached by an another animal that is perceived as a potential threat, possibly functioning to startle, confuse, frighten (Janzen et al 2010), threaten, and/or otherwise intimidate a predator. Indeed the sudden appearance of eyespots likely augments their protective value (Vallin et al. 2005, 2006). In contrast, (and occasionally in concert), some species possess constantly displayed eyespots that also appear to prevent attack, possibly through predator fright or intimidation (Karplus \& Algom 1981; Kodandaramaiah et al. 2009; Janzen et al. 2010).

Naturalists have long recognized that a variety of Lepidoptera caterpillars possess eyespots, and that some of these species closely resemble the eyes of snakes, lizards, mammals and birds (Bates 1862; Weismann 1882; Poulton 1890; Janzen et al. 2010). Such resemblance may be generated morphologically and/or behaviourally by various traits acting independently or synergistically to convey the impression of a snake or other predatory vertebrate. Henry Walter Bates described his encounter with one such caterpillar as "The most extraordinary instance of imitation I ever met with..." (Bates 1862, p.509). As naturalists continue to discover additional species, and describe 
previously undocumented larval stages of known species, it has become apparent that eyespots are frequent among tropical caterpillars. This is illustrated graphically in Janzen et al. (2010), as well as in images in the Área de Conservación Guanacaste (ACG) caterpillar database (Janzen \& Hallwachs 2014).

It seems to be generally accepted that caterpillar eyespots mimic the eyes of a snake or other threatening vertebrate (e.g., Edmunds 1974; Pough 1988; Lederhouse 1990; Janzen et al. 2010), thereby causing a predator to flee or hesitate in a strike, giving the caterpillar an escape opportunity (e.g., by dropping off the host plant or by simply causing the potential predator to not return to the microlocation of the caterpillar to verify its initial reaction; Janzen et al. 2010). The natural history of tropical birds and caterpillars argues strongly that the plethora of caterpillars with eyespots is the product of natural selection imposed by a set of avian predators, encompassing a variety of species, that innately respond to eye or face-like stimuli by adopting a range of risk mitigation measures including hesitation and abruptly fleeing (Janzen et al. 2010). The ubiquitous danger associated with close proximity to a predator's eyes (i.e., regardless of predator species) has likely favoured the generalized avoidance of eye-like features by birds (Janzen et al. 2010). That the markings on a given caterpillar do not seem to mimic any specific model predator (Rothschild 1984; Pough 1988; Janzen et al. 2010), in contrast to the frequent case with Batesian and Müllerian wing-pattern mimicry, further supports this idea.

Many of the most impressive, and perhaps best known, eyespot caterpillars are members of the family Sphingidae (Weismann 1882; Poulton 1890; Edmunds 1974; Janzen et al. 2010). Caterpillars from this group can be as long as $10 \mathrm{~cm}$ and $2 \mathrm{~cm}$ in 
diameter, and possess a distinctive mid-dorsal horn on the posterior end of their body (segment A8). This horn has sometimes been modified to be an eyespot or hardened button-like structure, especially in the caterpillar's ultimate instar (Heinrich 1979; Wagner 2005).

As part of ongoing research in Costa Rica we closely examined a Eumorpha phorbas (Cramer 1775) caterpillar (Sphingidae: Macroglossinae). In the ultimate instar this caterpillar's caudal horn has been reduced to a single eyespot capable of movement such that it appears to "blink" when the caterpillar is approached or harassed. That is, the fleshy exoskeleton around the posterior eye-like spot contracts then is quickly released creating what looks like a blinking eye. E. labruscae (Linnaeus 1758) is a closely related species collected previously in the same and adjacent forests in ACG (see below), and is similarly capable of "blinking" the posterior eyespot during the final instar (DHJ, personal observation). What follows is a description of the E. phorbas and E. labruscae caterpillars, our thoughts on the function of a "blinking" eyespot, and the implications of these observations for eyespot function. While this is the first published description of $E$. phorbas caterpillars (but see multiple images at Janzen and Hallwachs 2014), the specimen descriptions provided here are focused on describing features linked to the caterpillars' anti-predator strategy rather than an attempt to describe taxonomically relevant features.

\subsection{Material and methods}

The Eumorpha phorbas caterpillar was collected and reared within Sector San Cristobal by parataxonomists based at the rain forest Estación San Gerardo (10.8809 N, - 
85.3887 W, elev. 575 m) of Área de Conservación Guanacaste (ACG), Costa Rica, as part of an ongoing long-term caterpillar inventory (Janzen et al. 2005; Smith et al. 2008; Janzen \& Hallwachs 2014). The specimen is indexed within the ACG caterpillar database (11-SRNP-2980; Janzen and Hallwachs 2014). As is standard protocol for rearing caterpillars for the project, the caterpillar was maintained in an inflated plastic bag along with fresh foliage of its host plant, in this case Sarcopera sessiflora (Marcgraviaceae), a canopy-level large woody vine (that was rendered accessible by the $25 \mathrm{~m}$ tall tree being uprooted). The description of the E. labruscae caterpillar is based on the following caterpillars collected within the dry forest Sector Santa Rosa of ACG and indexed in the same database (but this species also occurs in ACG rain forest): 78-SRNP-35, 84-SRNP1316, 87-SRNP-560, 92-SRNP-1514.

Preliminary observation had revealed that the ultimate instar of the E. phorbas caterpillar "blinked" its posterior eyespot in response to tactile stimulus and to motion (See video in ESM 1). Here, a "blink" is characterized by a quick localized depression of the posterior section of the eyespot, resulting in a greater portion of the black "button" being revealed. To gain further insight into how this caterpillar might respond to an attacker, we carefully removed the caterpillar from its rearing bag and after approximately 1 minute of acclimation we prodded the specimen three times on the anterior segments; each prod was separated by $\sim 5-6 \mathrm{~s}$. The caterpillar was then placed back into its rearing bag with trimmings of its host plant. After $1 \mathrm{~h}$, the specimen was again removed and similarly prodded on the posterior end. Notes on the behaviour of $E$. labruscae are based on observations of specimens made previously by DHJ and WH in 
the course of the ongoing inventory of the ACG between 1978-1992 (see dates in rearing records at http://janzen.sas.upenn.edu).

\subsection{Results}

\subsubsection{Description of Eumorpha phorbas caterpillar}

Penultimate instar. The body is largely a uniform green colour that (to human eyes) qualitatively matches the foliage of its host plant. The lateral sides of the caterpillar are further textured with small irregular-shaped blue-white dashes nearly all of which occur above the spiracles (see images of 11-SRNP-2980 at http://janzen.sas.upenn.edu). The contrast of these markings is enhanced by dark charcoal-coloured edging which surrounds each marking, and they appear similar to small spots of insect damage on a leaf. One pair of such markings is particularly distinct and occurs on each side of the first abdominal segment. A red anal horn protrudes from the posterior margin of a single dorsal eyespot on the A8 segment; this eyespot is composed of a dark oval-shaped outer ring surrounding a single dark central spot. Both the inner and outer margins of the outer ring are highlighted by a lighter green, and the area between the ring and the central spot is the same green as the body, having transitioned from the black-brown colour of the central spot (Figure 5-1A). This caterpillar in nature perches on the leaves themselves when diurnally resting (Elda Araya, personal observation).

Ultimate instar. The specimen was $60 \mathrm{~mm}$ in length and $12 \mathrm{~mm}$ in diameter at the middle of the body. When undisturbed the caterpillar appears to human eyes especially cryptic against a naturally heterogeneous brown bark-colored background. We assume that 
during the daytime, this caterpillar rests on the tree bark under the epiphytic viney food plant, as is the case with other ACG caterpillars with this color pattern. Dorsally, the caterpillar's colouration was dominated by light and dark brown patterning (Figure 51B). The first abdominal segment possessed a pale-yellow cross marking dorsally (Figure 5-2A), whereas abdominal segments possessed textured green stripes extending towards the dorsal midline (Figure 5-1B). A pair of small cream coloured spots $(1.6 \mathrm{~mm} \times 0.7$ $\mathrm{mm}$ ) appeared on most of the abdominal body sections, located dorsal-laterally adjacent to the green markings (Figure 5-1B). Additional textured green markings bordered a reasonably sharp contrast between the caterpillar's dorsal and lateral colouration, where a much lighter brown patterning, with flashes of light green and dark brown that predominate on the lateral side of the abdominal segments (Figure 5-1B). When this color morph pulls its head into its thorax and expands the thorax, the frontal view is that of an arboreal viper such as the dark morph of Bothrops schlegeli (see images DHJ493714 through DHJ493719 for voucher code 11-SRNP-2980 at Janzen and Hallwachs, 2014).

However, an equally striking feature is the posterior eyespot of the ultimate instar. The characteristic anal horn of a sphingid caterpillar has been modified into a shiny black, hardened "button" partially covered by fleshy tissue. This "button" is located within an oval-shaped marking $(3 \mathrm{~mm} \times 3.5 \mathrm{~mm})$ surrounded by a thin black border and otherwise filled with a dark cream colour (Figure 5-2B). The eyespot also contains an additional small, black, oval-shaped marking $(0.8 \mathrm{~mm} \times 0.4 \mathrm{~mm})$ in its center (Figure 52B). 


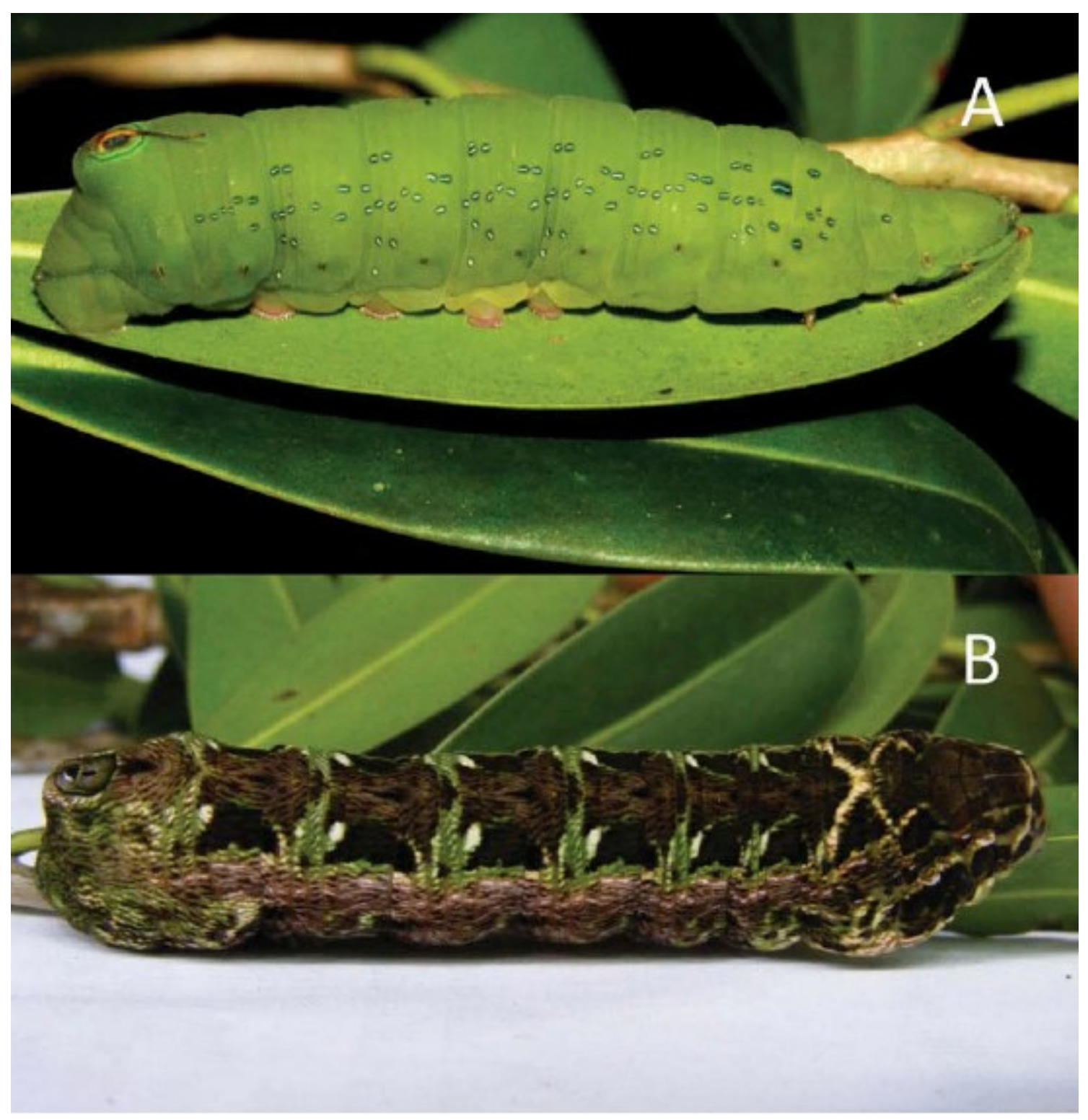

Figure 5-1: Lateral view of Eumorpha phorbas caterpillar during the penultimate (A) and ultimate (B) instars. 


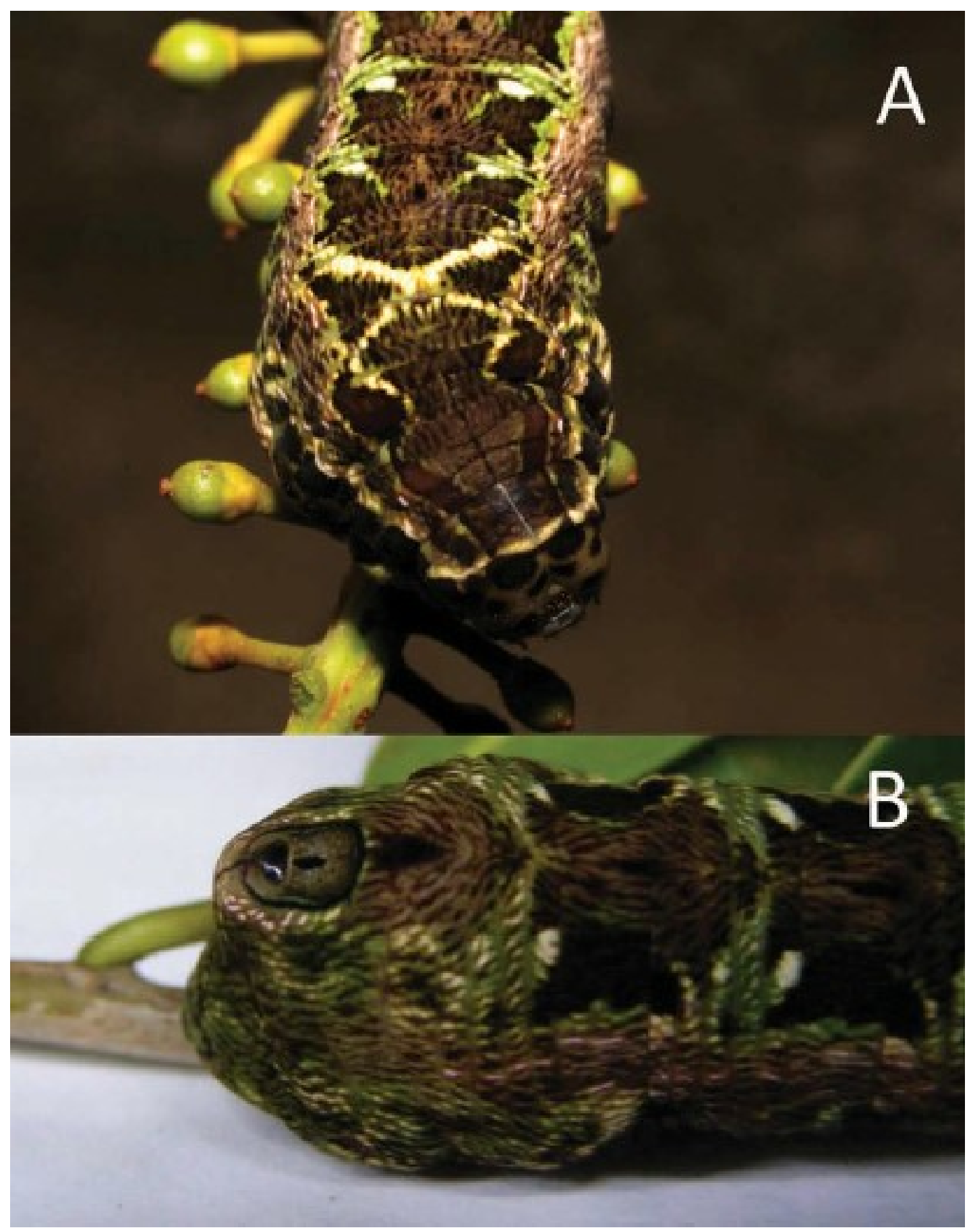

Figure 5-2: Close-up views of Eumorpha phorbas caterpillars. (A) Dorsal view of the anterior body segments, (B) dorso-lateral view of posterior eyespot which replaces the anal horn present in the penultimate instar. 
Behaviour. When initially disturbed, the caterpillar pulls its head into its thorax, expanding the thorax thus rendering it much like the head of an arboreal crypticallycoloured viper, with somewhat developed eyespots on the thorax (see above). When in this defensive posture both terminal ends are wider than the mid-body sections, the anterior reaching a diameter of $14 \mathrm{~mm}$ and the posterior end widening to a diameter of 13 $\mathrm{mm}$. The caterpillar thrashed its anterior body laterally in response to the first anterior prod, and "blinked" in response to all three anterior prods (see video in ESM 2).

Similarly, the caterpillar flicked its posterior body in response to the first posterior prod, and "blinked" in response to all three prods (see video in ESM 3). Notably, "blinks" were more frequent and rapid in response to the posterior prods than to the anterior prods (14 vs. 6 "blinks" in total; see ESM 2-3).

\subsubsection{Description of Eumorpha labruscae caterpillar}

Penultimate instar. In contrast to the penultimate instar of E. phorbas, the body of $E$. labruscae penultimate instar is largely a textured brown colour with green pear-shaped ovals on the flanks of the abdominal segments A2-A7 and distinct eyespots on the first abdominal segment (A1, Figure 5-3A). The dorsal body colour is, on average, a darker brown than the flanks and the lateral sides of the thoracic segments are coloured light yellow-brown. The combination of these colors renders the caterpillar cryptic when on tree bark, where it rests when not feeding on the foliage of its viney food plant. The eyespots on the A1 segment occur just above the spiracle and are composed of a black ring surrounded by a highly contrasting light yellow ring, with a dark white-speckled center. In addition, there is a distinct white spot within the black eyespot just above, and 
anterior to, the center. The long red anal horn extends upwards from the posterior margin of a black spot on the A8 segment (Figure 5-3A). This spot is surrounded by a highly contrasting orange ring, which is in turn bordered by a dark brown ring (Figure 5-3A).

Ultimate instar. About $80 \mathrm{~mm}$ long and with a similar colour pattern to that of $E$.

phorbas. The eyespots on segment A1 are present but less distinctive in comparison with the penultimate instar (Figure 5-3B). The distinct black ring remains, inside of which is a gray-brown base colour. The white spot remains within the center of each eyespot and is itself surrounded by a fine black ring (Figure 5-4A). An additional white spot may occur within the eyespot (e.g., images of 92-SRNP-1514 at Janzen and Hallwachs 2014). The eyespots contain several smaller small oval markings, which also occur on segment A-1 outside of the eyespot (Figure 5-4A). The dorsal colour of abdominal segments A2-A7 is similar to that of E. phorbas, but E. labruscae is distinct in having short light yellowbrown streaks that extend from the anterior margin of each segment (Figure 5-3B). A pair of small faint spots can be seen on the dorsal side of segments A1-A7, towards the posterior side of each segment. The anal horn on segment A8 is reduced to a hardened "button" and is similar to that of the ultimate instar E. phorbas (Figure 5-4B). It differs in being more circular, having an outer pale yellow ring, and containing an additional internal ring that surrounds the hardened button (Figure 5-4B). 


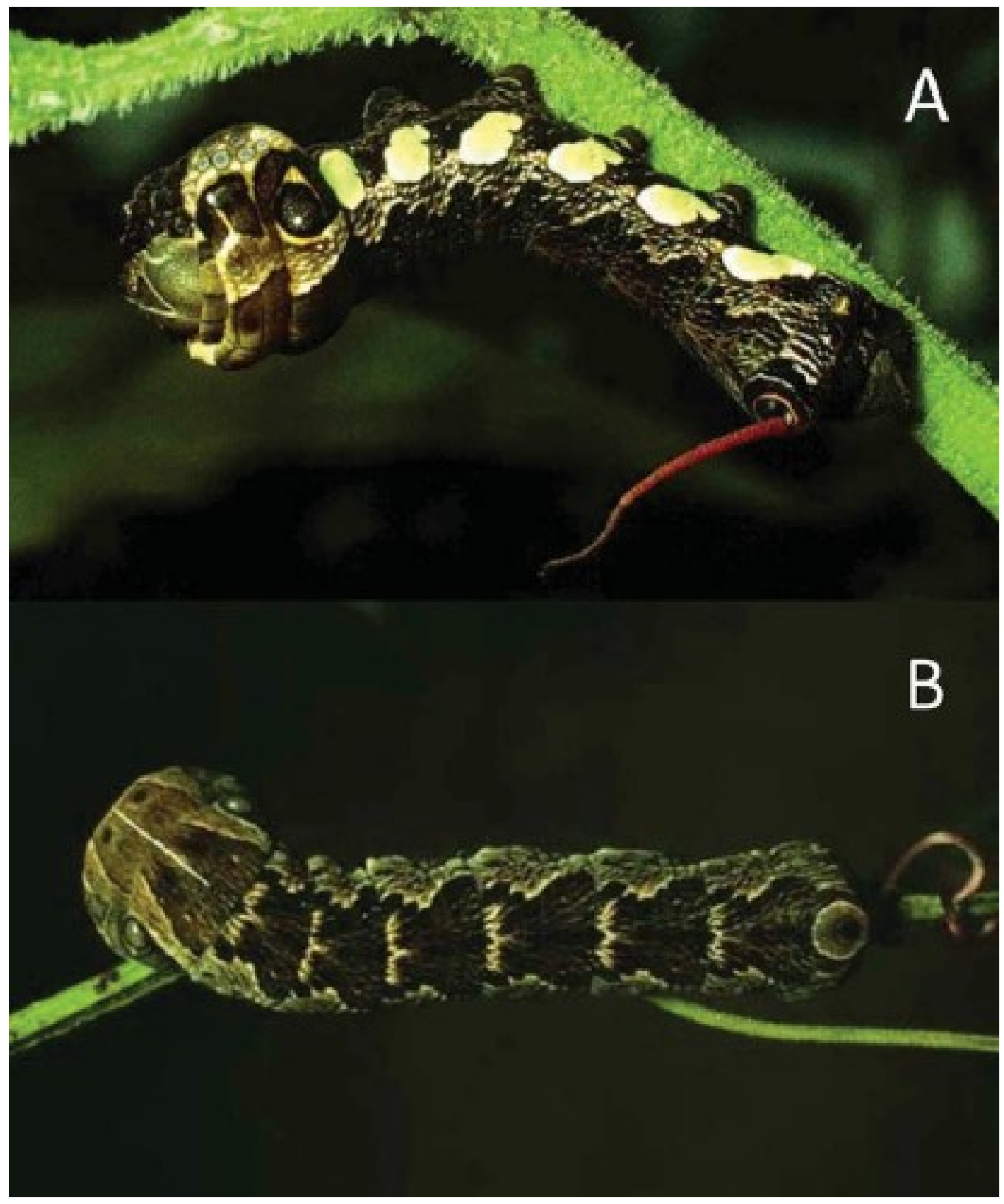

Figure 5-3: Lateral view of Eumorpha labruscae caterpillar during the penultimate (A) and ultimate (B) instars. 


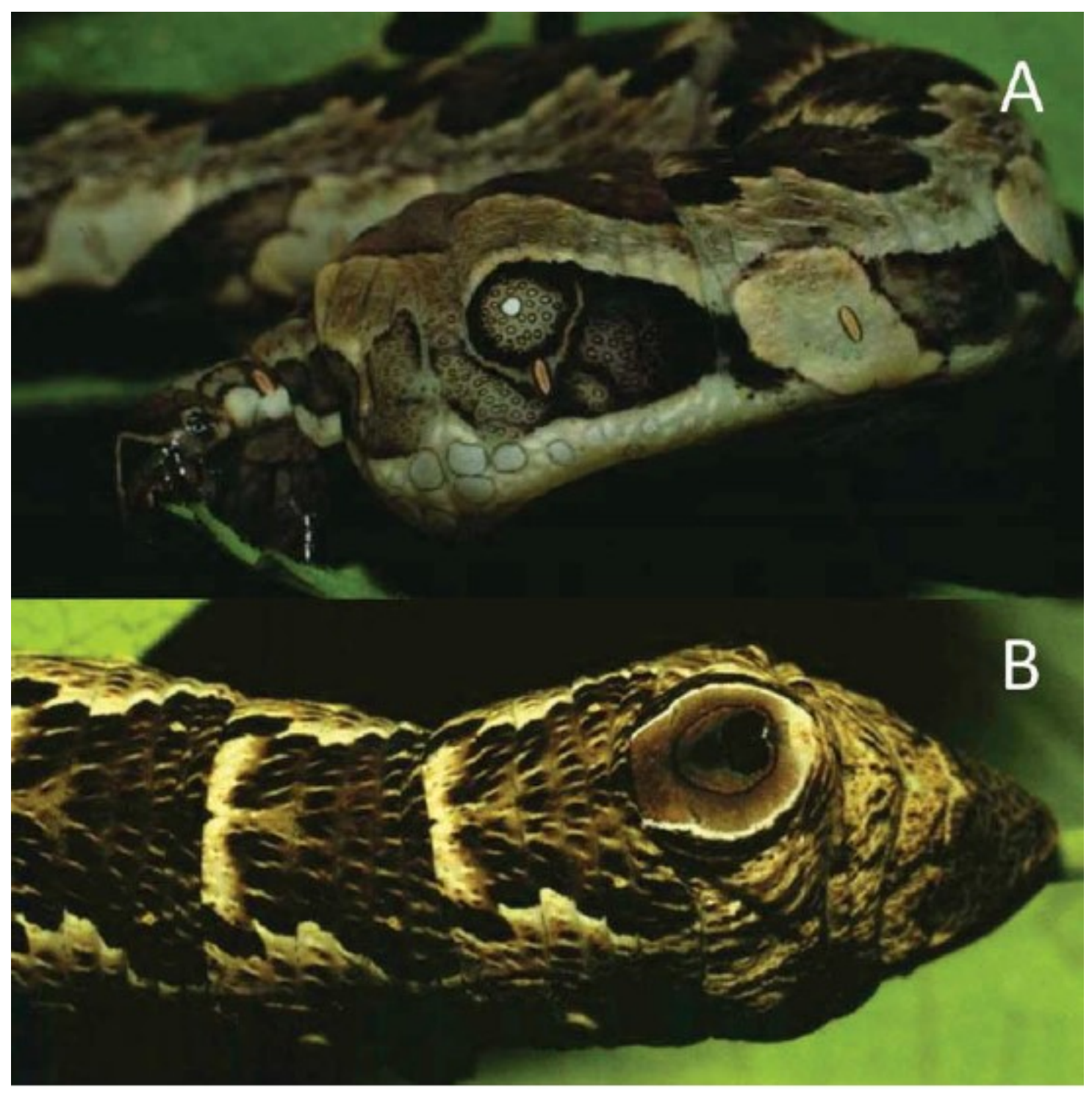

Figure 5-4: Close-up views of Eumorpha labruscae caterpillar. (A) Lateral view of the anterior body segments, (B) dorsal view of posterior eyespot that which replaces the anal horn present in the penultimate instar. 
Behaviour. When harassed both the ultimate and penultimate instars of this caterpillar have an effective snake-like display during which they telescopically pull the head into the thoracic segments, which in turn inflates the caterpillar's anterior segments. This behaviour creates a shape similar to that of a snake's head and increases the conspicuousness of the pair of eyespots on the first anterior body segment. Penultimate instar caterpillars flick the red anal horn when harassed, whereas the ultimate instar "blinks" the posterior eyespot.

\subsection{Discussion}

We have described the Eumorpha phorbas and E. labruscae caterpillars which, during the ultimate instar, respond to close approach and tactile stimulation by contracting a localized portion of its posterior eyespot; to human observers such palpitation produces the effect of a blinking eye. Eyespot "blinking" involves a constantly displayed eyespot which appears to close and open and is thereby distinct from well-known cases where concealed eyespots are suddenly revealed upon attack (e.g. the eye spots on the wings of many Lepidoptera). Although, suddenly revealing otherwise hidden eyespots may produce a greater startle effect, a "blinking" eyespot likely mimics a real vertebrate eye more closely. When approached or harassed, the described Eumorpha caterpillars also inflate their thoracic segments to form a diamond shape similar to the head shape of dangerous, co-occurring snakes. These traits may work independently or in combination with eyespots to deter predators from attacking once they have detected the caterpillar and the facultative expression of these traits likely minimizes any costs 
associated with signal expression (e.g., via reduced crypsis, energetic expenditure or lost foraging opportunity).

The rear eyespot is only contracted upon close approach or harassment, suggesting that "blinks" are directed towards a perceived threat and we suggest that that this "blinking" is an extension of eye mimicry. Quickly concealing/revealing the dark central "pupil" of an eyespot upon harassment ought to make a more convincing "eye". Furthermore, the response of a small insect-eating bird that finds itself very close to what appears to be a blinking eye would almost certainly be to distance itself from that object (Janzen et al. 2010). As such, it seems to us that the "blinking" behaviour described here likely protects the caterpillar from attackers by mimicking a vertebrate eye.

Both E. labruscae and E. phorbas caterpillars possess a long red anal horn during the penultimate and pre-penultimate instars, attached just posterior to the site of the hardened button (Fig. 5-3A, 5-4A). When disturbed during the pre-penultimate instar, $E$. labruscae (and perhaps also E. phorbas) caterpillars rapidly beat or whip this long anal horn (Moss 1912). Whipping the anal horn may fend off parasites (Moss 1920), intimidate attackers by resembling a snake tongue (Curio 1965), or function as part of a generalized startle display. The ability to "blink" the rear eyespot during the ultimate instar is likely produced by the same musculature used formerly for whipping the anal horn in the intermediate instars. As the observed specimens were part of the ongoing ACG inventory project, which involves rearing to adulthood, lethal sampling and dissection was not possible.

Other published accounts of a "blinking" eyespot include Moss (1912) who describes the ultimate instar of Pholus labruscae (now: Eumorpha labruscae, the same 
species as observed in ACG) collected in Peru, Hayward (Hayward 1929) who described an ultimate instar P. labruscae (E. labruscae) caterpillar from Villa Ana, Argentina, and Curio (1965) who described an ultimate instar P. labruscae (now: E. labruscae yupanquii) collected on Isla Santa Cruz (Indefatigable) in the Galápagos. All three descriptions emphasize the snake-like appearance of the anterior segments, as well as the posterior spot that oscillates with muscular action. Notably, Moss (1912) suggests that both ends of the caterpillar resemble the head of a snake, and Hayward (1929) observed that the caterpillar "...is reminiscent of certain poisonous snakes, and the natives refuse to touch it".

Interestingly, the posterior spot on the ultimate instar of E. labruscae was described by Moss (1912) as “...capable of rapid palpitation, catching the light and flashing like a serpent's eye when molested". However, although snakes are, almost exclusively, cited as the model for caterpillars with eyespots (e.g., Pough 1988; Lederhouse 1990), snakes have transparent eye scales ("brilles") in place of eyelids and therefore do not blink or flash (Mead 1976). It is unclear whether Moss' use of "flashing" refers to the blinking action of the spot, or simply that the white spot appears that there is light reflected from a black spot as it would a snake's eye. We emphasize that for small insect-eating birds the ubiquitous threat associated with proximity to an eye suggests that eyespots need not mimic the eye of any specific model species to provide some degree of protection (Janzen et al. 2010). A fear response would be similarly generated by the fear of other co-occurring lizards, mammals and birds that do blink and also pose a considerable threat to insect-eating birds. Since the anterior end of E. labruscae and $E$. phorbas possesses features that mimic a snake, and the "blinking" spot on the posterior 
end mimics the eye of a different enemy (suggested also by Curio 1965), such a feature likely extends the range of predators that can be deterred from attack upon detecting caterpillar.

To date, E. phorbas and E. labruscae and are the only species known to be capable of "blinking" an eyespot, and are the only Eumorpha species known to have evolved this specialized posterior eyespot. It remains to be seen whether E. capronnieri, a closely related species, also possess this specialized posterior eyespot. The other reared ACG Eumorpha (E. obliquus, E. satellitia, E. vitis, E. megaeacus, E. fasciatus, E. triangulum, E. anchemolus) are all cryptic green to yellow to pink in coloration with various sizes of white diagonal side slashes, but lack the this specialized "blinking" eyespot (see images at Janzen \& Hallwachs 2014). We propose that the "blinking" eyespot observed in these final-instar Eumorpha caterpillars intimidates would-be predators from attack. However the extent to which caterpillar predators perceive the rear eyespot as an eye blinking and the degree of protection conferred remains unknown. We are not aware of any reported observations of bird responses to these caterpillars. Unfortunately, the rarity of these Eumorpha species currently precludes direct examination of ecologically-relevant predators responding to the "blinking" eyespots of these caterpillars. Future collection of E. labruscae for more detailed study may be 
possible outside of its primary habitat where it is ephemerally abundant (but of course where also the relevant predators may be absent).

\subsection{Acknowledgments}

We thank Tim Caro and an anonymous referee for helpful comments on our manuscript. We are grateful to ACG parataxonomists Elda Araya, Carolina Cano, Osvaldo Espinoza and Gloria Sihezar for finding and rearing this Eumorpha phorbas caterpillar and Adrian Guadamuz for identifying its food plant; equally we are grateful to ACG Parataxonomists Ricardo Calero, Dinia Martinez, Petrona Rios, Calixto Moraga, Manuel Rios and Freddy Quesada for finding the second Eumorpha phorbas caterpillar and rearing it to adulthood. The study has been supported by U.S. National Science Foundation grant 0515699, and grants from the Wege Foundation, International Conservation Fund of Canada, Blue Moon Fund, Guanacaste Dry Forest Conservation Fund, Área de Conservación Guanacaste, University of Pennsylvania (DHJ and WH) and NSERC Discovery (TNS). 


\section{Chapter: Body size affects the evolution of eyespots in caterpillars}

Thomas John Hossie ${ }^{1 *}$, John Skelhorn ${ }^{2}$, Jesse W. Breinholt ${ }^{3}$, Akito Y. Kawahara ${ }^{3}$, and Thomas N. Sherratt ${ }^{1}$

${ }^{1}$ Department of Biology, Carleton University, Ottawa, ON, Canada

${ }^{2}$ Institute of Neuroscience, Newcastle University, Framlington Place, Newcastle, UK

${ }^{3}$ Florida Museum of Natural History, University of Florida, Gainesville, FL, USA

*Corresponding author

In preparation for submission to Proceedings of the National Academy of Sciences USA

Short Title: Large caterpillars are more likely to evolve eyespots

Classification: Biological Sciences: Evolution

Keywords: Lepidoptera, pastry caterpillars, Gallus gallus domesticus, mimicry, predatorprey, antipredator defence, Sphingidae 


\subsection{Abstract}

Many caterpillars possess conspicuous eye-like markings, commonly called eyespots. These markings have long captivated the interest of biologists, and despite recent work demonstrating their efficacy in deterring avian predators a satisfactory explanation for why some species have eyespots while others do not has not been forthcoming. We propose that caterpillars which attain a large body size are more likely to evolve these markings because (i) large prey are innately conspicuous, (ii) large prey are more profitable, and/or (iii) eyespots are more intimidating to predators when on large-bodied prey. Using a phylogenetically-controlled analysis, we show that large body size is indeed associated with the possession of eyespots, at least in Macroglossinae (Sphingidae). To evaluate the proposed mechanisms we subjected model caterpillars arranged in a $2 \times 2$ factorial design (eyespots vs. no-eyespots; small vs large) to predation by wild birds in the field. These same treatments were also offered to naïve domestic chicks in a controlled lab setting where we measured search time, inspection time, and overall latency to attack. As predicted, eyespots increased predation rates on small prey both in the field and the lab, but only intimidated chicks when on the large-bodied prey. Large prey with eyespots survived equally well as large prey without eyespots in the field indicating that eyespots did not further enhance the conspicuousness of large prey. Thus, both selection against eyespots in small caterpillars and for eyespots in large caterpillars has led to the observed eyespot-body size patterns we see in extant species. 


\subsection{Significance statement}

Eyespots are an important and widespread antipredator defence, particularly within Lepidoptera, and have been recognised as such for over 150 years. In caterpillars, eyespots are thought to intimidate insect-eating birds from attack because they possess an innate fear of predator eyes. Yet, to date an explanation for why eyespots occur in some species but not others has not been forthcoming. This work combines a phylogeneticallycontrolled analysis, a field experiment with wild birds, and a lab experiment with naïve chicks to provide the most comprehensive investigation of the phenomenon to date. Our phylogenetic work demonstrates that large body size is closely associated with the evolution of eyespots, while our experimental work reveals the selection pressures that led to this relationship. 


\subsection{Introduction}

Predation is a strong selective force acting to shape animal phenotypes and numerous traits have evolved to reduce the probability of predator detection or attack (Edmunds 1974; Ruxton et al. 2004a). For caterpillars, one important and remarkably widespread adaptation is the possession of conspicuous eyespots (Lederhouse 1990; Janzen et al. 2010). These markings caught the attention of several eminent evolutionary biologists including Henry Walter Bates (Bates 1862), August Weismann (Weismann 1882) and E. B. Poulton (Poulton 1890), each of whom understood them to be defensive features mimicking the eyes of a dangerous vertebrate and thereby intimidating would-be predators from their attack. This idea was treated more formally by Pough (Pough 1988) who described these caterpillars as snake mimics explicitly. Recently, Janzen et al (Janzen et al. 2010) updated and expanded this explanation, further suggesting that the aversion to eye- or face-like stimuli is innate and that there is no particular selection for eyespots to resemble the eye of any specific model. Subsequent work has now empirically confirmed the protective value of eyespots to caterpillars (Hossie \& Sherratt 2012,2013 ) and shown that the mimetic fidelity of many caterpillars with eyespots to dangerous snakes increases when they adopt their defensive posture (Hossie \& Sherratt 2014); although some research has questioned the degree to which "eyespots" confer protection through eye mimicry at least in adult Lepidoptera (Stevens 2005; Stevens \& Ruxton 2014). If eyespots are beneficial in deterring predation, then one might wonder why they are not seen in all caterpillars. However, to date there has been no formal identification of the species which have eyespots and those that do not (but see Prudic et 
al. 2007), let alone a test of why these features have evolved in some species and not others.

A mounting body of research is attempting to relate anti-predator adaptations to other aspects of the species' life history or habitat (e.g., Lovegrove 2001; Hagman \& Forsman 2003; Prudic et al. 2007; Hultgren \& Stachowicz 2009; Stankowich \& Caro 2009; Penney et al. 2012; Rudh 2013). For example, the evolution of aposematism in Papilio caterpillars was unrelated to diet or chemical specialization (as observed in poison arrow frogs; Santos et al. 2003; Darst et al. 2005), but was instead strongly influenced by host plant architecture, with conspicuous warning signals selected in species that feed on host plants where it is difficult to hide (i.e., narrow-leaved plants) (Prudic et al. 2007). Similarly, Stankowich and Caro (Stankowich \& Caro 2009) demonstrated that large body size and living in open habitats, both of which increase conspicuousness, are related to the possession of weaponry (i.e., horns) in female bovids. Large body size has also been associated both with reduced investment in camouflage in crabs (Hultgren \& Stachowicz 2009), and more recently with an increase in mimetic fidelity in hover flies (Penney et al. 2012). In caterpillars that live fully exposed to potential predators, eyespots seem to occur primarily on relatively large species (although eyespots do also occur on many small caterpillars that feed in leaf shelters) (Janzen et al. 2010). In addition, many of the most impressive examples of putative snake mimic caterpillars, such as Deilephila elepenor, Hemeroplanes ornatus, Madoryx plutonius, and various Xylophanes $s p$., are comparatively large (Weismann 1882; Poulton 1890; Moss 1920). Indeed, even in the above species, eyespots are substantially reduced or absent altogether during the early instars and tend not to become prominent until the late instars 
when the caterpillars are much larger (Weismann 1882; Janzen \& Hallwachs 2014). Taken together this suggests that the selective advantage of eyespots to caterpillars may depend on their body size.

We see three main non-mutually exclusive reasons for selection to favour eyespots more strongly in large caterpillars. First, large animals are innately more conspicuous and may therefore face relatively strong selection favouring the evolution of defences that operate after detection. Small animals on the other hand are better able to go undetected by predators and either do not experience strong selection for secondary defences, or face selection against conspicuous markings that break crypsis. Similar arguments have suggested previously that mimicry is more likely to evolve in species that are already moderately conspicuous (Charlesworth \& Charlesworth 1975; Gilbert 1991; Speed \& Ruxton 2010). Second, selection may favour eyespots more strongly in large caterpillars because large prey represent relatively profitable prey items to their predators. Both the incentive to locate a given prey item, and to then discriminate edible mimics from dangerous or inedible models upon discovery, should increase with prey size. Consequently, prey with increasingly large body size may experience progressively stronger selection for effective secondary defences (Penney et al. 2012). Finally, eyespots may confer a greater advantage to large caterpillars because their size enables them to convincingly resemble something that poses a legitimate threat (such as a snake). In this way, large body size itself may act as an exaptation (Gould \& Vrba 1982), predisposing large caterpillars to the evolution of deceptive traits like eyespots.

Herein we have examined the relationship between body size and eyespots by conducting: i) the first phylogenetically-controlled assessment of the association between 
larval body size and the possession of eyespots in caterpillars, ii) a field experiment using caterpillar models of different sizes exposed to predation by wild birds, and iii) a lab experiment where similar caterpillar models were presented to naïve domestic chicks. A phylogenetically-controlled analysis was essential to conservatively control for any of lack of independence of species data arising through shared ancestry. The field experiment directly tested whether the protective value of eyespots is conditional on large body size, and staged presentations in the lab enabled us to quantify the effects of body size and eyespots on prey detection and predator intimidation. Thus, by combining phylogenetic, field, and lab approaches we comprehensively investigated whether the evolution of eyespots in caterpillars is size-dependent, and tested hypotheses as to why this might be so.

\subsection{Results}

\section{Phylogenetic comparison}

The phylogenetic generalize least squares (PGLS) model using an OrnsteinUhlenbeck correlation structure $($ alpha $=12.49)$ was a significantly better fit than the model assuming Brownian motion $\left(\triangle \mathrm{AIC}=3.22\right.$; Likelihood Ratio Test, $\chi^{2}=5.22, P=$ 0.02). There was a significant relationship between maximum body size in the final larval instar and the presence of eyespots in Macroglossinae caterpillars $\left(\mathrm{t}_{63}=2.26, P=0.03\right.$, Figure 6-1). Phylogenetic signal in the residuals (Pagel's $\lambda$ ) was estimated at 0.698 . The phylogeny that we used had high node support and the topology was identical using maximum likelihood or Bayesian methods. 


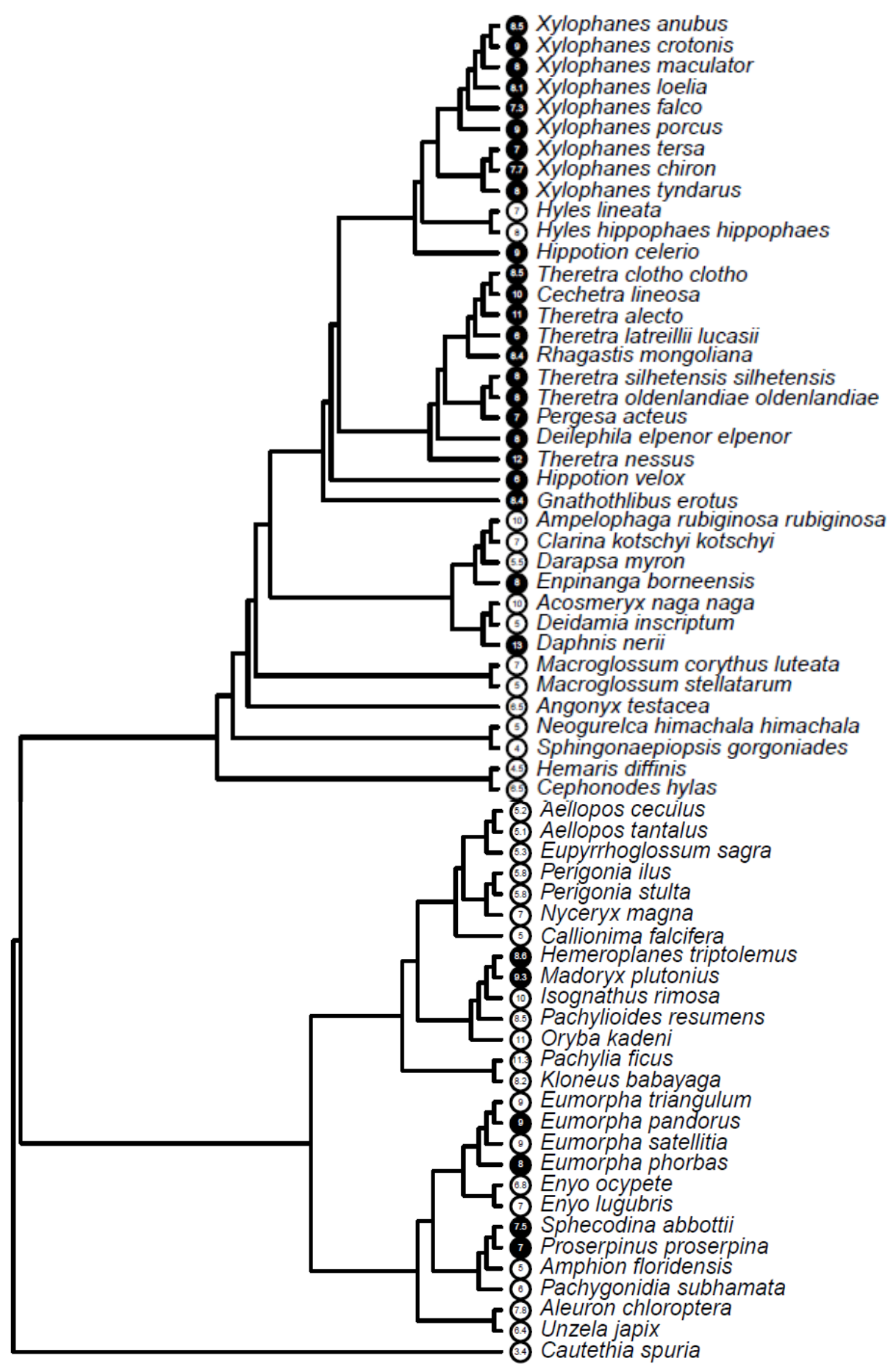

Figure 6-1: Phylogeny of Macroglossinae (Sphingidae) species included in the analysis. Black symbols indicate the species whose caterpillars possess eyespots and the number inside indicates the caterpillar's maximum body length $(\mathrm{cm})$ at the larva's final instar. 


\section{Field presentations}

The $90 \mathrm{~h}$ mortality rate of artificial caterpillars ranged from $24 \%$ to $97 \%$ per location (mean \pm SE: $68.5 \pm 4.52 \%$ ). Hazard rate (i.e., mortality rate) was significantly influenced by the main effects of both body size $(z=3.04, P=0.002)$ and eyespots $(z=$ 2.24, $P=0.025)$, but we did not detect a significant interaction $(z=-1.04, P=0.30)$. Possessing a larger body size increased hazard by $37 \pm 14.8 \%$, and possessing eyespots also appeared to increase hazard overall by $23.7 \pm 14.9 \%$. Yet, closer inspection revealed that this eyespot-induced increase in hazard only significantly affected small prey $(26.3 \%$ increase, Wald $=6.45, P=0.011)$, and did not affect large prey (5.61 increase, Wald $=$ $0.36, P=0.55)$. Therefore, eyespots strongly hinder the survival of small prey, but did not affect survival of large prey (Fig. 6-2). Classifying only prey recovered with peck marks as killed (i.e. considering all missing prey as censored) produced qualitatively similar results, but with a stronger effect of body size $(251.6 \pm 28.26 \%$ increase in hazard, $\mathrm{z}=4.45, P<<0.001)$, and a non-significant effect of eyespots $(\mathrm{z}=0.93, P=$ $0.35)$. The interaction was also non-significant $(\mathrm{z}=-0.74, P=0.46)$. This difference from the original model resulted from a greater proportion of small prey being completely removed upon attack $\left(F_{3,20}=4.44, P=0.015\right.$; small-no eyespots: $83.2 \pm 1.85 \%$ removed completely, small-eyespots: $80.8 \pm 2.19 \%$, large-no eyespots: $48.7 \pm 5.00 \%$, largeeyespots: $49.5 \pm 4.60 \%)$. 


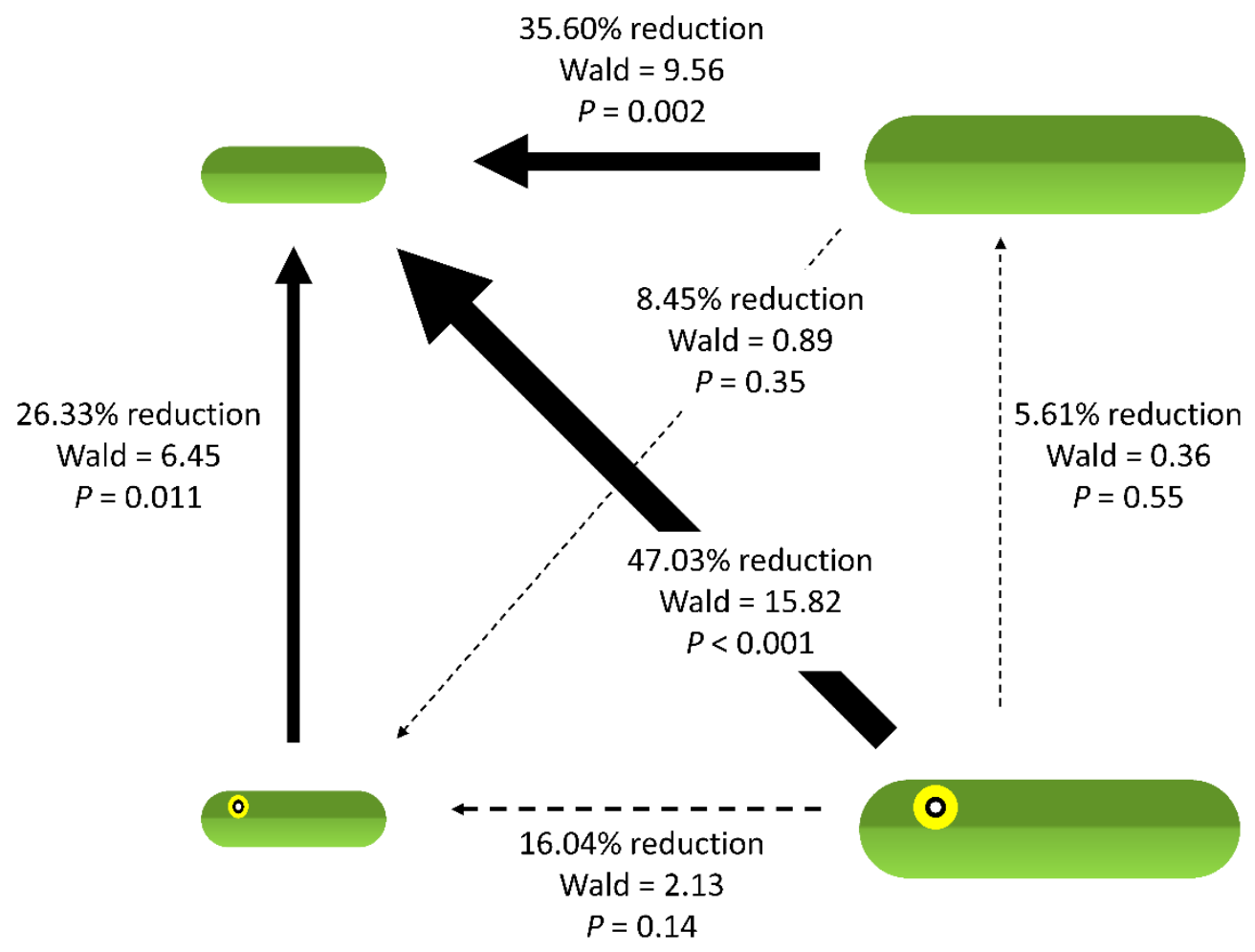

Figure 6-2: Results from pairwise Cox proportional hazard regression comparisons of pastry caterpillar survival in the field experiment. Eyespot (i.e. no eyespots versus eyespots present) and body size (i.e. $2 \mathrm{~cm}$ versus $4 \mathrm{~cm}$ in length) treatments were arranged in a $2 \times 2$ factorial design. Arrow width is proportional to the relevant Wald statistic and point towards the treatment with lower hazard rate. Solid arrows are significant at $\mathrm{P}<0.05$. 


\section{Lab presentations}

We observed significant main effects of body size $\left(\mathrm{F}_{1,36}=37.33, \mathrm{P}<0.001\right)$ and eyespots $\left(\mathrm{F}_{1,36}=15.36, \mathrm{P}<0.001\right)$ on the latency to attack (total time from introduction to attack), as well as a significant body size $\times$ eyespots interaction $\left(\mathrm{F}_{1,36}=66.04, \mathrm{P}<\right.$ 0.001). The presence of eyespots had opposite effects on the latency to attack in small and large prey (Fig. 6-3). Specifically, chicks took a longer time to attack large prey with eyespots (mean \pm SE: $305 \pm 37.30 \mathrm{~s})$ compared to large-no eyespot prey $(198.5 \pm 19.53 \mathrm{~s}$; Tukey HSD: $\mathrm{P}=0.026$ ), but a relatively short time to attack small prey with eyespots $(49.8 \pm 6.96 \mathrm{~s})$ compared to small-no eyespot prey $(240.5 \pm 12.16$; Tukey HSD: $\mathrm{P}<$ 0.001). Interestingly, the latency to attack was not significantly different between smallno eyespot prey and both large-no eyespot and large-eyespot prey (Tukey HSD: P $=0.49$ and $\mathrm{P}=0.42$, respectively). Search time (time to first inspection) was affected by the main effect of eyespots $\left(\mathrm{F}_{1,36}=26.71, \mathrm{P}=0.001\right)$ and the body size $\times$ eyespot interaction $\left(\mathrm{F}_{1,36}=38.57, \mathrm{P}<0.001\right)$, but the main effect of body size was not significant $\left(\mathrm{F}_{1,36}=\right.$ $3.38, \mathrm{P}<0.07)$. In the absence of eyespots, search time was greater for small prey (smallno eyespots: $236.3 \pm 12.44$ s, large-no eyespots: $160.4 \pm 19.84$ s; Tukey HSD: $\mathrm{P}=0.019$ ), and the addition of eyespots to small prey substantially reduced search time (smalleyespots: $38.8 \pm 6.69 \mathrm{~s}$; Tukey HSD: $\mathrm{P}<0.001)$. In contrast, adding eyespot to large prey did not increase the search time (large-eyespots: $178.5 \pm 24.73 \mathrm{~s} ; \mathrm{P}=0.88$ ). Unexpectedly, large-eyespot prey had a longer search time than small-eyespot prey (Tukey HSD: $\mathrm{P}<0.001)$, but not small-no eyespot prey $(\mathrm{P}=0.11)$. Body size, eyespots and their interaction significantly affected inspection time $\left(\mathrm{F}_{1,36}=142.63, \mathrm{P}<0.001, \mathrm{~F}_{1,36}\right.$ $=16.43, \mathrm{P}<0.001$, and $\mathrm{F}_{1,36}=20.84, \mathrm{P}<0.001$, respectively). The inspection time did 
not differ between small prey with and without eyespots (small-no eyespots: $4.2 \pm 0.84$, small-eyespots: $3 \pm 0.58$; Tukey HSD: $\mathrm{P}=0.98$ ), yet eyespots significantly increased inspection time in large prey (large-no-eyespots: $38.1 \pm 5.24$, large-eyespots: $127.1 \pm$ $24.05 \mathrm{~s}$; Tukey HSD: $\mathrm{P}<0.001)$. Chicks took longer to inspect large prey even when they lacked eyespots (Tukey HSD: P $<0.001$ ). Finally, body size and eyespots had nonindependent effects on approach-retreat behaviour $\left(X^{2}=22.93, \mathrm{df}=4, \mathrm{P}<0.001\right)$. Specifically, the behaviour was never seen in trials with small prey, but was observed in $20 \%(2 / 10)$ of the large-no eyespot trials and in $80 \%(8 / 10)$ of the trails with largeeyespot prey. 


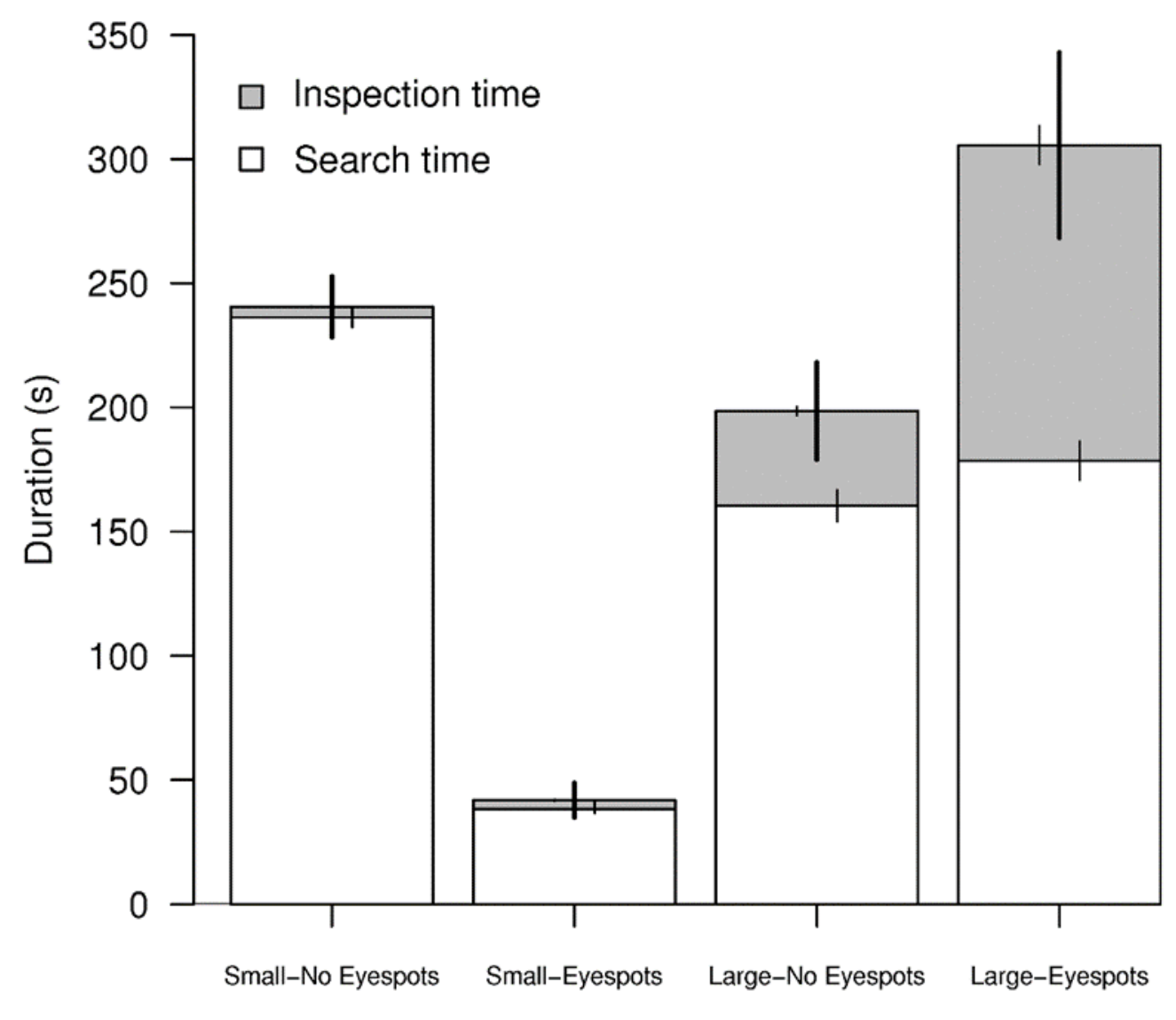

Figure 6-3: Results from the lab experiment using domestic chicks. Bars represent the latency to attack broken up into search time (i.e., time to first monocular inspection), and inspection time (i.e., time from first inspection to attack). Ten chicks were exposed to each treatment. Vertical lines on each horizontal bar represent the estimated standard error (SEM). The thickened vertical lines represent the SEM for overall latency to attack. 


\subsection{Discussion}

Our results provide strong evidence that body size has influenced the evolution of eyespots in caterpillars. By examining traits of Macroglossinae (Sphingidae) caterpillars, and controlling for the effects of common ancestry, we found that species whose larvae attain relatively large body size in their final instar tend to have eyespots. To examine the mechanisms underpinning this relationship we subjected artificial caterpillars of different sizes to predation by wild birds in the field, and to domestic chicks in the lab. In the field, large prey had lower survival than small prey indicating either that large body size increases relative conspicuousness or that predators preferentially target large prey because they are more profitable. The survival of small, but not large, prey in the field was hindered by the addition of eyespots suggesting that the protective value of eyespots is size-dependent. In the lab, small prey without eyespots took the longest to find, and adding eyespots to these prey severely reduced search time (along with overall latency to attack). In contrast, even without eyespots large prey were intimidating to chicks, and yet adding eyespots to large prey delayed attack even further though an additional increase ( $\sim 3$ fold) in time from detection to attack. Moreover, eyespots induced approach-retreat behaviour in chicks only when they were on large prey, emphasizing that the ability of eyespots to intimidate predators is dependent on prey body size. Taken together, this work illustrates that both selection against eyespots at small body sizes, as well as selection for eyespots at large body sizes, have likely been important in generating and 
maintaining the observed size-dependent distribution of eyespots across species and ontogeny.

Mimetic species are generally thought to descend from cryptic ancestors (Charlesworth \& Charlesworth 1975) and it remains somewhat unclear how mimicry can arise gradually from crypsis (Kikuchi \& Pfennig 2009). Specifically, how do relatively poor mimics balance the cost of increasing their conspicuous to predators during the initial stages of evolving mimicry? This paradox is resolved when low mimetic fidelity can secure significant survival benefits (Kikuchi \& Pfennig 2009), or if animals have sufficient incentive to break crypsis anyway (e.g., through active foraging) thereby minimizing the effects of reductions in morphological crypsis (Gilbert 1991). Our field experiment revealed that increasing body size substantially increases the predation risk for cryptic prey, and similar results reported elsewhere have been attributed to associated increases in detection by a predator (Remmel \& Tammaru 2009). Alternatively, such increases in risk with body size could be attributed to selection for larger, more profitable prey (Penney et al. 2012). Interestingly, we also found that in large prey there was no cost associated with possessing conspicuous eyespots. Therefore, the costs which might otherwise impede the evolution of conspicuous markings in cryptic morphs are likely low enough in large, easy to detect, caterpillars to facilitate the evolution of eyespots even if their protective value is only moderate. This is not the case in small caterpillars however, where eyespots impose large costs in terms of increased detectability that are not counterbalanced by a protective effect thereby precluding the evolution of such markings.

Both our field and lab experiments show that eyespots do not effectively intimidate predators when possessed by small prey, yet a clear intimidation effect of 
eyespots was observed in the lab when large prey with eyespots were presented to chicks. The onset of approach-retreat behaviour in chicks upon their spotting the large prey models with eyespots makes it harder to believe that the increase in the chicks' latency to attack results from dietary conservatism, neophobia, or avoiding of prey with conspicuous signals (Stevens 2005; Stevens \& Ruxton 2014), rather than from mimicry of snakes or other dangerous vertebrates as many have suggested (Pough 1988; Janzen et al. 2010; Hossie \& Sherratt 2014). It is unlikely that large prey (with or without eyespots) took longer to detect than small prey with eyespots. Instead, we suspect that our lab experiment overestimated the proportion of the overall latency to attack allocated to "search time" for large prey because the time to true first detection is not accurately represented by latency to first monocular inspection when prey are conspicuous and intimidating. Although previous experiments using model caterpillars have observed a protective effect of eyespots (Hossie \& Sherratt 2012, 2013), large prey with and without eyespots did equally well in our field experiment. We suspect that the wider array of avian predators in the field, a subset of which are less fearful of eyespots or prioritize different features as cues of danger (e.g., "head" shape, Hossie \& Sherratt 2013, 2014; or sinuous movements, Janzen et al. 2010), renders the protective value of eyespots more variable or moderate in natural settings. Moreover, experiments using stationary models are likely to underestimate the protective value of eyespots to large caterpillars which enhance these signals though behavioural mimicry.

Although eyespots occur in large species that live fully exposed (and primarily during the larger, late instars of those species), in other families within Lepidoptera (e.g., Hesperiidae) they also occur occasionally on small species whose larvae feed inside leaf 
shelters (Janzen et al. 2010). Our work shows that eyespots would actually be detrimental to small prey that live fully exposed because eyespots increase conspicuousness without making them intimidating to predators. However, by inhabiting leaf shelters small prey would be able to minimize the conspicuousness cost while also maximizing the 'startle' effect of these markings and making it more difficult for a bird to estimate their body size. Eyespots did not seem to increase the conspicuousness of large prey, yet some large species with eyespots conceal their eyespots within folds of their cuticle (e.g. Madoryx plutonius; Janzen et al. 2010). This might indicate that under natural conditions there is still selection for large prey to minimize the conspicuousness of these markings.

An important exception to the body size-eyespot relationship we observed is the frangipani hornworm (Pseudosphinx tetrio), which is also a member of Macroglossinae. The caterpillar can attain a maximum larval body length of $11.3 \mathrm{~cm}$ (11-SRNP-57021, Janzen \& Hallwachs 2014), and lacks eyespots. Instead of having eyespots this caterpillar appears to be aposematic (Merle et al. 2001; Sloan et al. 2006; Dunford \& Barbara 2008; Marsh \& Rothschild 2009), and may even mimic coral snakes (Janzen 1980), clearly having found a distinct way to solve the problem of being a large bodied prey item. Curiously, within Sphingidae, larval eyespots seem to be restricted to Macroglossinae and it remains unclear why this would be the case. It is not immediately apparent what might constrain the evolution of eyespots in these subfamilies, when many of Smerintinae and Sphinginae larvae are large (Janzen \& Hallwachs 2014), and eyespots appear to have evolved multiple times within Macroglossinae. Also, Papilio caterpillars have evolved eyespots (and "snake-like" defensive postures; Hossie \& Sherratt 2014) even though they are much smaller than the eyespotted Macroglossinae caterpillars 
examined here, and many of them live fully exposed. It has been proposed that pigmented and forked defensive structure that can be everted from behind the head of Papilio caterpillars (i.e. the osmeterium) might resemble a snake's tongue (Pickens 1927), perhaps making up for their small size and pre-adapting them to snake mimicry.

Eyespots have attracted attention from naturalists and evolutionary biologists for over 150 years, and herein we provide an explanation for why eyespots in caterpillars seem to be restricted to large species, late instars, and small species inhabiting leafshelters. In essence it is because eyespots tend to be selected against in small continuously exposed prey, but favoured in larger prey through their size-dependent intimidatory effect, which offsets any associated minor increase in conspicuousness. The next step is to explain the observed variation in mimetic fidelity among these species (Penney et al. 2012). This system poses a unique challenge however because unlike many classical model-mimic systems these caterpillars seem to be exploiting an innate wariness to their own enemies (Pough 1988; Janzen et al. 2010). Despite this difficulty it should still be possible to quantify mimetic fidelity, perhaps by measuring the responses of naïve or trained birds to photographs (Dittrich et al. 1993) or by using techniques that don't assume any single model species (Hossie \& Sherratt 2014). This system seems particularly amenable to examining the generality of several interesting patterns recently reported in other mimetic taxa. For example, are higher levels of mimetic fidelity required on the edge of the model's range, as observed in coral snakes (Harper \& Pfennig 2007)? Does mimetic fidelity correlate with body size, as observed in hoverflies (Penney et al. 2012)? Is behavioural mimicry (e.g., highly sinuous movements, mock striking) more likely to evolve in high fidelity mimics (Penney et al. 2014)? Future investigations 
of these questions will undoubtedly extend our understanding of Batesian mimicry and should work to include mimicry complexes where avoidance of the model is innate.

\subsection{Materials and methods}

Phylogenetic comparison

We collected data on maximum body size and the presence or absence of eyespots in final instar Sphingidae caterpillars from a range of reliable online databases, field guides, and other publications (detailed in Table S6-1). Interestingly, all the examples of caterpillars with eyespots came from the Macroglossinae subfamily. There is substantial overlap in body size among the larvae of all three subfamilies, suggesting that the evolution of eyespots is prevented in Smerinthinae and Sphinginae caterpillars by a constraint other than body size. This is an interesting observation in itself, but not the focus of our work. We thus restricted our analyses to Macroglossinae. Using a molecular phylogeny (built using maximum likelihood methods by JWB and AYK) we tested the hypothesis that larger caterpillars are more likely to have eyespots. Specifically, we conducted a phylogenetic generalized least squares (PGLS) analysis with body size as the response variable and the presence/absence of eyespots as a binary predictor (factors are not permitted as response variables in PGLS). We ran PGLS models assuming either a Brownian motion or Ornstein-Uhlenbeck (Martins \& Hansen 1997) model of evolution then compared their fits using a likelihood ratio test (Paradis \& Claude 2002). To ensure that accounting for phylogenetic autocorrelation was necessary, we tested whether the residuals from a linear model without phylogenetic control showed phylogenetic signal 
(Revell 2010) using the function phylosig in the R package phytools (Revell 2012).

Artificial prey

Artificial caterpillars were designed to quantify the selective advantage of eyespots in large and small prey. Manufacture of artificial caterpillars followed methodology similar to previous studies (Hossie \& Sherratt 2012, 2013). For ecological relevance our models were based loosely on Papilio canadensis and Papilio glaucus caterpillars. Caterpillar models comprised four treatments: small-no eyespots; large-no eyespots; small-eyespots; large-eyespots. All treatments were similarly countershaded, because Hossie and Sherratt (Hossie \& Sherratt 2012) revealed that eyespots effectively protected these artificial caterpillars from birds only when the caterpillars were also countershaded.

Pastry caterpillars were manufactured with a 3:1 mixture of white flour and lard. Light green pastry was produced by adding three drops of Leaf Green AmeriColor ${ }^{\mathrm{TM}}$ Soft Gel Paste ${ }^{\mathrm{TM}}$ (AmeriColor Corp., Placentia, CA, U.S.A.) and $20 \mathrm{ml}$ of water to $40 \mathrm{ml}$ of lard and $118 \mathrm{ml}$ flour, whereas six drops of gel paste and $15 \mathrm{ml}$ of water were added to the same volumes of flour and lard to produce the dark pastry. Dark and light pastry was rolled into strips, $3.5 \mathrm{~mm}$ in diameter for small prey and $5 \mathrm{~mm}$ in diameter for large prey, which were then cut into lengths of $20 \mathrm{~mm}$ or $40 \mathrm{~mm}$ for small and large prey respectively. Two-toned (i.e. countershaded) prey were then created by pressing together one piece of dark and light pastry such that the final artificial prey were cylinders $20 \mathrm{~mm}$ long and $5 \mathrm{~mm}$ in diameter for small prey, or $40 \mathrm{~mm} \times 7 \mathrm{~mm}$ for large prey. For our 
purposes the dark side was considered dorsal as prey were presented on the upper side of branches.

We used the same eyespot design as in Hossie \& Sherratt (Hossie \& Sherratt 2013). Eyespots were hand-painted onto pastry caterpillars using Elmer's ${ }^{\mathrm{TM}}$ Non-toxic Tempera Paint (Elmer's Products, Inc., Westerville, OH, U.S.A.). For the large prey, eyespots were $4.5 \mathrm{~mm}$ in diameter, $4 \mathrm{~mm}$ apart, and $7 \mathrm{~mm}$ from the anterior tip of the prey. The eyespots had a $3 \mathrm{~mm}$ black 'pupil', with a $1 \mathrm{~mm}$ white 'sparkle'. For the small prey, eyespots were sized proportional to their body size. Specifically, eyespots were 2.2 $\mathrm{mm}$ diameter, $2 \mathrm{~mm}$ apart and $3.5 \mathrm{~mm}$ from the anterior tip. The black 'pupil' was 1.5 $\mathrm{mm}$, and the white 'sparkle' was $0.5 \mathrm{~mm}$ in diameter. A dorsal view of the eyespot treatments is provided in appendix Fig. S6-1. Finished pastry caterpillars were checked against a standard model prey for each treatment to ensure uniformity in size and shape of the body, as well as the size and position of eyespots. These artificial prey were used in both field presentations to wild birds and the lab presentations to domestic chicks.

\section{Field presentations}

Field trials were conducted at three sites in Ontario, Canada (site 1: near the Ferguson Forest Centre, Kemptville, $45^{\circ} 03^{\prime} 00^{\prime} \mathrm{N}, 7^{\circ} 39^{\prime} 44^{\prime \prime} \mathrm{W}$; site 2: the University of

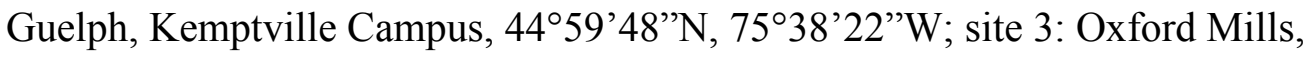
$\left.44^{\circ} 56^{\prime} 27^{\prime} \mathrm{N}, 75^{\circ} 38^{\prime} 56^{\prime \prime} \mathrm{W}\right)$. Experiments were run between 7 May and 7 September 2013. All three sites were composed of secondary growth mixed-deciduous forest and no 
site was closer than $5.5 \mathrm{~km}$ to another site. Insectivorous bird species observed in each of the field locations are detailed in appendix E Table S6-2.

Pastry caterpillars were deployed between 1600 and 1900 hours on the first day of a replicate site. Specifically, 24 trees were selected along a linear transect ( $\sim 10 \mathrm{~m}$ apart), and to each tree a single artificial prey was pinned on the upper side of a live branch in the north, south, east and west zone of a tree. Trees were restricted to one of four species (Populus tremuloides, Populus deltoides, Betula papyrifera, Acer rubrum). Each tree received a single pastry caterpillar from each treatment and branch zone (i.e. north, south, east, west), allocated randomly. Selected branches were 1-2 $\mathrm{m}$ from the ground and were roughly $0.5-1 \mathrm{~cm}$ in diameter. Pastry caterpillars were considered killed if they were no longer present or had been attacked by a bird (i.e., peck marks present). 'Survival' was checked at $14,19,24,38,43,48,62,67,74$ and $90 \mathrm{~h}$ from deployment. In all cases of missing baits, the pin was found and the ground underneath was searched for any remaining pieces. All attacked prey were photographed then removed. Artificial prey damaged by rain, slugs or ants were removed and considered censored (i.e. considered to have survived only to that time period, but not considered killed) in the final analysis. After $90 \mathrm{~h}$, remaining prey were removed.

The entire process was repeated six times for a total of 576 artificial prey deployed. Each consecutive replicate was conducted in an entirely new location within one of the three larger sites and was at least $200 \mathrm{~m}$ from any previous location within that site. To avoid temporal confounds among sites, consecutive replicates were always 
conducted at a different site in a systematic order (i.e. site 1, location 1; site 2, location 1; site 3 , location 1 ; site 1 , location 2 , etc.).

\section{Lab presentations}

Forty-eight domestic chicks of the 'Ross' strain (Gallus gallus domesticus) were purchased from a commercial hatchery on the day they hatched. They were housed in cages measuring $120 \mathrm{~cm}$ x $50 \mathrm{~cm}$ x $50 \mathrm{~cm}$, and were subject to a $14 \mathrm{hrs} \mathrm{light} \mathrm{:} 10 \mathrm{hrs}$ dark daily cycle using florescent lights with full daylight spectrum. The temperature of the laboratory was maintained at $24-27^{\circ} \mathrm{C}$ using room heaters. Water was provided $a d l i b$., as were chick starter crumbs, except during training and experimenting when brief periods of food deprivation were necessary to ensure chicks were motivated to forage. All deprivation periods were in accordance with U.K. Home Office regulations and guidelines. At the end of the experiments chicks were euthanized by dislocation of the neck as outlined in the Code of Practice for the Humane Killing of Animals under Schedule 1 to the Animals (Scientific Procedures) Act 1986.

On day 1 post-hatch, chicks were left to acclimatize to the laboratory for one hour. After this, 40 experimental chicks were trained to eat chick starter crumbs from the white paper floor of an experimental arena. Two identical arenas were used, each consisting of a cage identical to the housing cages, with a section measuring $20 \mathrm{~cm} \times 50$ $\mathrm{cm} \times 50 \mathrm{~cm}$ partitioned off using wire mesh to create a separate 'buddy arena'. In all training and experimental trials, two chicks were placed in the buddy arena to reduce any potential distress from placing experimental chicks alone in the arena. These buddy 
chicks were selected from a stock of 8 individuals, and were changed every three trials. These chicks were not given access to artificial prey at any point during the experiment. Chicks underwent six training trials at regular intervals: three on day 1 post-hatch and three on day 2. In all trials, chick crumbs were scattered over the floor of the experimental arena, and chicks were then placed in the arena for an interval of $2-3$ minutes. In the first two trials, chicks were placed in the arena in groups of three; in the following two trials, chicks were placed in the arena in groups of two; and in the final two trials chicks were placed in the arena individually. In the final trial a $50 \mathrm{~cm}$ long field maple (Acer campestre) branch complete with leaves was placed in the centre of the arena to familiarize chicks with branches in order that they did not show a neophobic response to the branch in the subsequent test trial (see below). Chicks were food deprived for 30 minutes before each of the training trials on day 2 to ensure they were motivated to forage. During all training trials, the two arenas were used simultaneously, but during the test trial only one arena was used so that chicks could be observed individually. By the end of trial 6, all chicks had pecked the branch, and were eating crumbs in the experimental arena.

On day 3 , birds were randomly assigned to one of 4 experimental groups $(n=10$ per group). Birds in all groups received a single test trial, in which they were placed in the experimental arena individually (as in training, two chicks from the stock of 8 were placed in the buddy arena in each trial) after 30 minutes of food deprivation. In the experimental arena each chick encountered a field maple branch $(50 \mathrm{~cm}$ in length) containing a single pastry caterpillar. This was placed in the center of the experimental arena with one end $25 \mathrm{~cm}$ from the buddy arena and the other $25 \mathrm{~cm}$ from the rear wall of 
the arena: chicks were placed in a central position immediately in front of the rear wall of the experimental arena. Caterpillars were attached to the branches using pins pushed into the branches in random positions. The tops of the pins were then cut off leaving approximately $3 \mathrm{~mm}$ protruding from the branches: pastry caterpillars were impaled on these. However, the type of caterpillar birds encountered differed between experimental groups: one group received a small caterpillar without eyespots; one a small caterpillar with eyespots; one a large caterpillar without eyespots; and one a large caterpillar with eyespots. Birds were left in the arena until they attacked the caterpillar. We recorded the latency to first inspect the caterpillar (i.e., tilt the head and view the caterpillar monocularly) and term it "search time". In addition, the latency to attack the caterpillar outright, and the presence/absence of approach-retreat behaviour (repeatedly approaching and retreating from the caterpillar) were recorded. We calculated "inspection time" as the total latency to attack minus search time. In this way we were able to separate the effects of our treatments on the processes of prey detection (which should affect search time) and predator intimidation (which should affect the time from prey detection to attack).

\section{Statistical analyses of field and laboratory experiments}

The survival of artificial prey in the field was analyzed using Cox proportional hazards regression (Cox 1972), which can handle both censored data and non-uniform changes in predation risk. We controlled for the possible lack of independence between baits on the same tree by clustering the baits by tree in our analysis. We assessed overall significance of the model using the Wald test, which does not assume independence of observations within a cluster. Factors in our model included body size (i.e. small versus 
large), eyespot (i.e. eyespots versus no eyespots) and the body size×eyespot interaction. The analysis was stratified to permit separate baseline hazard rates for location and tree species. This analysis was conducted in R (R Development Core Team 2014) using the survival library (Therneau 2014), and the assumption of proportionality was tested using the cox. zph function.

Latency to attack (s), search time, and inspection time in the chick experiment were each analysed using separate factorial ANOVAs, with body size, eyespots and the body size $\times$ eyespot interaction as factors. Analyses were conducted in $\mathrm{R}(\mathrm{R}$ Development Core Team 2014). Latency to attack and inspection time were square root transformed to meet ANOVA assumptions. The presence/absence of approach-retreat behaviour was analyzed using a three dimensional chi square test with body size (large, small), eyespots (presence, absence), and approach-retreat (presence, absence) as the dimensions.

\subsection{Acknowledgements}

We thank Dan Janzen and Winnie Hallwachs for the opportunity to observe several tropical caterpillars in the field. Funding for this research was provided by a Discovery Grant (NSERC) awarded to TNS. 


\section{Chapter: General conclusions and future work}

\subsection{Thesis contributions and conclusions}

This thesis represents the most comprehensive treatment of the adaptive significance of caterpillar eyespots to date and attempts to lift the phenomenon out from the largely anecdotal and just-so style treatment it has received for decades. I have provided important empirical support for untested hypotheses related to how eyespots confer protection from predators and uncovered several novel insights as well. Specifically, the first step to understanding eyespots as an adaptation is to show that they have an effect on the fitness of animals which possess them. While some research on the adaptive value of eyespots in adult models had been conducted, before my work the contention that eyespots could affect survival of caterpillars in the field was largely conjecture, with limited support coming largely from anecdotal and poorly replicated studies. Chapters 2, 3, and 6 addressed this shortfall by showing that eyespots can enhance the survival rate of artificial caterpillars subject to predation, under at least some conditions. In addition, both of the comprehensive theoretical treatments of the phenomenon to date (i.e. Pough 1988; Janzen et al. 2010) propose that eyespots (among other features) confer protection to caterpillars because they exploit an innate, unlearned fear in birds. Chapter 6 experimentally demonstrated that eyespots clearly intimidate naïve domestic chicks from their attack, though successful intimidation is contingent on the caterpillar's body size.

Many view eyespotted caterpillars as snake mimics (e.g., Pough 1988; Wagner 2005), yet no study had ever attempted to quantify mimetic fidelity. A likely impediment to this was that no one caterpillar has a single, definable model while many different 
traits simultaneously contribute to what we might consider mimetic fidelity. By developing a means to quantify mimetic fidelity, I tested the widely held belief that the defensive posture creates a more snake-like head shape in the anterior body segments of the caterpillar (Chapter 4). I showed not only that these subjective beliefs are backed up by more objective measures of mimetic fidelity, but also that these defensive postures enhance survival (Chapter 3). Although snakes may play a primary role as the model in this system (as suggested by Pough 1988, and Chapter 4), Janzen et al (2010) made it clear that the eyes of other dangerous vertebrates likely play a role in generating or reinforcing the fear of eye-like features in the predator population. Chapter 5 illustrates this clearly by describing two caterpillars which possess a posterior eyespot that "blinks" upon harassment. Given that snakes do not blink (they have eye scales called "brilles" in place of eyelids) these "blinking" eyespots are clearly not directly mimicking the eye of a snake, but may instead mimic some other vertebrate eye. These same caterpillars also have a defensive posture that makes them appear more snake-like from the anterior end (analogous to those described in Chapter 4). I suggest that mimicking features from multiple distinct dangerous vertebrates may serve to protect these caterpillars from a wider array of predators.

Perhaps most importantly, my work provides an answer to the fundamental, yet overlooked, question of why some caterpillars evolve eyespots while others do not (Chapter 6). Phylogenetic evidence indicated that the presence of eyespots in those caterpillars that feed fully exposed can be explained principally by body size, with larger caterpillars being more likely to possess eyespots. To follow up on the mechanisms driving this association I conducted both a field experiment with wild birds and a lab 
experiment with naïve domestic chicks. These experiments show that the body sizeeyespot relationship emerges as a result of selection against eyespots in small prey, and for eyespots in large prey. Specifically, eyespots are intimidating to birds only when they occur on large caterpillars, and they incur substantial conspicuousness costs only to small caterpillars that are not already east to detect (Chapter 6). In my opinion, the value of this work is increased substantially by having the empirical support from my previous chapters (especially Chapters 2-4) which provide the requisite evidence that caterpillar eyespots contribute to fitness by increasing survival.

During the course of this research there have been several insights that I did not anticipate. For example, the efficacy of caterpillar eyespots seems to be affected by body colour (Chapter 2), and the defensive posture was equally protective for caterpillar models with and without eyespots (Chapter 3). Similarly unexpected was the observation that strong selection against eyespots in small caterpillars may be critically important in generating the eyespot-body size relationships we observe among species and across ontogeny (Chapter 6). While I had not predicted them, in retrospect each of these patterns are clearly consistent with what we observe in nature; caterpillars with eyespots are invariably cryptic and often countershaded, many caterpillars lacking eyespots seem to possess (or retain) snake-like defensive postures, and small caterpillars either lack eyespots or have eyespots and feed in leaf-shelters to minimize the conspicuousness cost of eyespots. Doubtless, many more unexpected results will emerge upon closer examination of this system, but each one will help us to develop a more complete view how these traits have evolved and currently operate. 


\subsection{Additional remarks on caterpillar eyespots}

Having worked on caterpillar eyespots for the last four years I have come to the general conclusion that in the majority of cases, eyespots on larval lepidopterans mimic the eyes of snakes or other dangerous vertebrates. This seems to be the only explanation that convincingly and naturally explains the following lines of evidence: 1) eyespots are located where you would expect them to be if they were mimicking eyes (Chapter 4); 2) defensive postures make the caterpillars look more snake like (Chapter 4); 3) naïve chicks only fear these traits when positioned in a typical head or face-like manner (Skelhorn et al, in prep); 4) rather than simply avoid eyespots and wide "head" shapes, birds seem to be actively afraid (Shirota 1980; Chapter 6, Skelhorn et al, in prep); 5) eyespots only work on large caterpillars, big enough to resemble a potential threat (Chapter 6); 6) especially convincing examples of snake mimicry exist (e.g., Hemeroplanes triptolemus) that appear to exploit the same features as other evolutionarily distinct examples of caterpillars with eyespots, suggesting widespread (taxonomically and geographically) convergent evolution. I therefore see caterpillars with eyespots explicitly in terms of Batesian mimicry, having been generated as a by-product of the innate fear that insect-eating birds possess for the eye- or face-like features of their own enemies.

I emphasize that not all circular markings on caterpillars will be selected through eye mimicry. As described in the general introduction, circular markings may in some cases have an aposematic function, a fact recognized early on by August Weismann (1882). In many cases the alternate function of circular markings may be apparent, however the distinction made between "eye-spots" and "ring-spots" by Weismann (1882) 
(i.e., that "eye-spots" have a "pupil" and mimic eyes, whereas "ring-spots" lack a pupil and are aposematic) may not always hold. Specifically, I would not expect that only circular markings that are pupillated could effectively mimic eyes. Given the high cost of failing to react quickly to a real threat combined with the speed at which predators must decide whether or not to flee, it is conceivable that features other than "pupils" could make markings sufficiently eye-like to elicit aversion (i.e., simply being circular, paired, and/or correctly placed on the body might be sufficient in many cases). Equally, I am reluctant to make the statement that pupillated spots could not have an aposematic or generalized "startle" function in some caterpillars, as a "pupil" could increase the salience of the marking to predators. In my opinion the function of such markings on a caterpillar is likely to be judged more reliably by the location of the marking (i.e., is it placed towards one end of the caterpillar?), and the body colour of the rest of the caterpillar (i.e., is the rest of the caterpillar cryptic or gaudy?). Specifically, a mimetic function is more likely when the markings are placed in the context of a head (towards one end of the caterpillar; Skelhorn et al, in prep), and when the rest of the caterpillar is cryptic (Chapter 2). Additional clues may come from how the caterpillar responds to harassment, and whether the caterpillar mounts a defensive posture akin to those described in Chapter 4.

\subsection{Ongoing and future work}

My research has attempted to take the phenomenon of caterpillar eyespots from a collection of passing observations and mature it into a system with a strong empirical foundation which can be used to deepen our understanding of mimicry and predator 
defense more generally. The groundwork is now laid for future investigators to employ caterpillars with eyespots as a means to push forward our understanding of predator psychology, imperfect mimicry, behavioural mimicry, and the evolutionary consequences of deception. Here I sketch out what I see as some of the more profitable directions for future research, some of which are currently ongoing.

\subsubsection{Mimicry versus conspicuous signals}

Strictly speaking, this thesis did not explicitly test whether eyespots and the defensive posture confer protection because they are eye- and head-like, or simply because these traits are novel and conspicuous (e.g., Stevens 2005). Stevens and Ruxton (2014) point out this interpretive limitation, stating explicitly that: "The authors did not modify the characteristics of the eyespots (e.g. shape, contrast, number, and so on) and so it is hard to draw conclusions about whether such spots work due to mimicry or conspicuousness". We are aware of this issue and have recently conducted experiments in collaboration with Dr. John Skelhorn (Newcastle University, UK) using naïve domestic chicks to address this point. Specifically, we examined whether chicks were equally deterred by eyespots and the defensive posture (i.e., the swollen body segments) if they were placed towards one end of the model (i.e., in a head-like context), or in the centre of the model (i.e. in a non-head-like context). These treatments were also compared to models lacking these features altogether. The head-like and non-head-like treatments were equally conspicuous, but differ in their resemblance to a head or face. Thus, if eyespots and the defensive posture confer greater protection when in the head-like context we can confidently be ascribe the protective value of these features to mimicry. 
This work was not yet submitted for external review when this thesis was submitted, but indeed found strong support for mimicry. Both eyespots and the defensive posture were only effective deterrents to naïve chicks when they occurred in the "head-like" context.

In this chick experiment described above, the effects of posture and eyespots were examined in separate experiments so we were unable to address the possible synergistic effect of both traits. Stevens and Ruxton (2014) suggested that such a synergistic effect was in important proof of mimicry, and we may investigate such effects in the future. However, I do not believe that such synergistic effects are the litmus test to identifying the mechanism through which these features work. If the traits work in isolation, and it can be demonstrated that protection is gained (from either feature) only when that feature is placed in a head- or face-like context, we are still able to soundly reject the conspicuous signal hypothesis. Failure to observe a subsequent increase in protection when both traits are present could result simply from prey reaching maximal protection from either trait alone, distinct subsets of the predator guild utilizing distinct features as predator cues, or overshadowing effects where predators use a few salient features to classify prey and ignoring other possibly informative features (Gamberale-Stille et al. 2012; Aronsson \& Gamberale-Stille 2012; see also (Valkonen et al. 2011a). In fact, Dr. David Kikuchi (NSF Fellow, Carleton University) is leading some ongoing work 
investigating such adaptive limits to learning and the consequences for mimicry in collaboration with my supervisor and collaborator Dr. Tom Sherratt.

\subsubsection{The role of behaviour}

Chapters 2, 3 and 6 demonstrated that eyespots on artificial caterpillars have an anti-predator function; however the protective effect of eyespots in the field was typically moderate. I have suggested throughout the thesis that the modest efficacy of eyespots in these experiments may be partially explained by the design excluding relevant antipredator behaviours (i.e., other than defensive posture). Indeed, prey behaviours that mimic the hostile behaviour of a snake (e.g., directed thrashing), produce smooth sinuous movements, or simply increase the chance that eyespots are seen might enhance a bird's fear response upon encountering a caterpillar with eyespots. Such behaviours seem to be widespread in 'snake mimic' caterpillars, yet it remains unclear the extent to which these behaviours increase the protective value of other mimetic features (or are even functionally linked to snake mimicry). Interestingly, human infants also have a predisposition to fear snakes, but movement seems to be necessary to for them to associate snakes with fear (Deloache \& Lobue 2009).

During the course of my thesis I made two explicit attempts to address the contribution of behaviour: first, by presenting live and freshly killed caterpillars with or without eyespots to wild birds (summer 2011, Figure 7.1); and second, by developing robotic caterpillar that would rear up upon tactile stimulus (summer 2013, Figure 7.2). Both projects unfortunately failed. I could not get birds to reliably visit the feeding platforms I had set up, and the activation mechanism in the model caterpillars was 
ineffective when the cord leading to the model was bent. I still see these projects as viable, simply requiring some reworking of the materials and methods. An obvious refinement for these experiments would be to conduct them in captivity with domestic chicks or wild-caught birds. The logistics involved in maintaining a population of live birds was beyond our capabilities, but this approach certainly remains a viable means forward for others who want to investigate the role of behaviour in the future. 


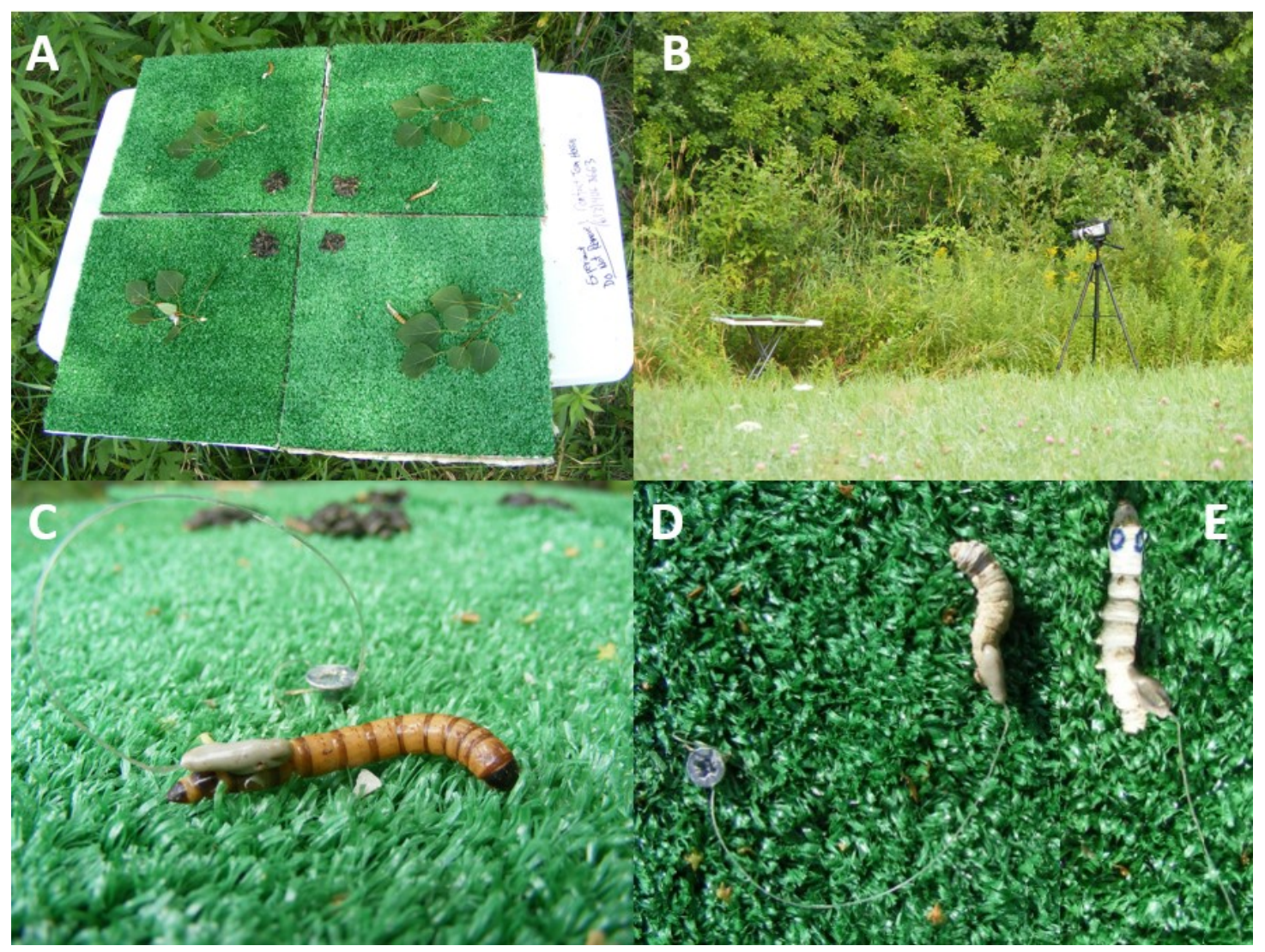

Figure 7-1: Experimental set up for a failed experiment in 2011 which set out to investigate bird responses to caterpillar eyespots and anti-predator behaviour (arranged as a $2 \times 2$ factorial). A) The feeding table during training phase. Live "superworms" (Zophobas morio larvae) tethered to screws using monofilament fishing line and hair-removal wax, and black sunflower seeds added to draw in birds and acclimate them to feeding on the table. B) The table and video camera set up in in field as viewed from the blind 20m away. C) Close-up of a tethered superworm D) Close-up of a tethered silkworm (Bombyx mori larvae), E) Tethered silkworm upon which eyespots were painted used as a preliminary test of the system. 


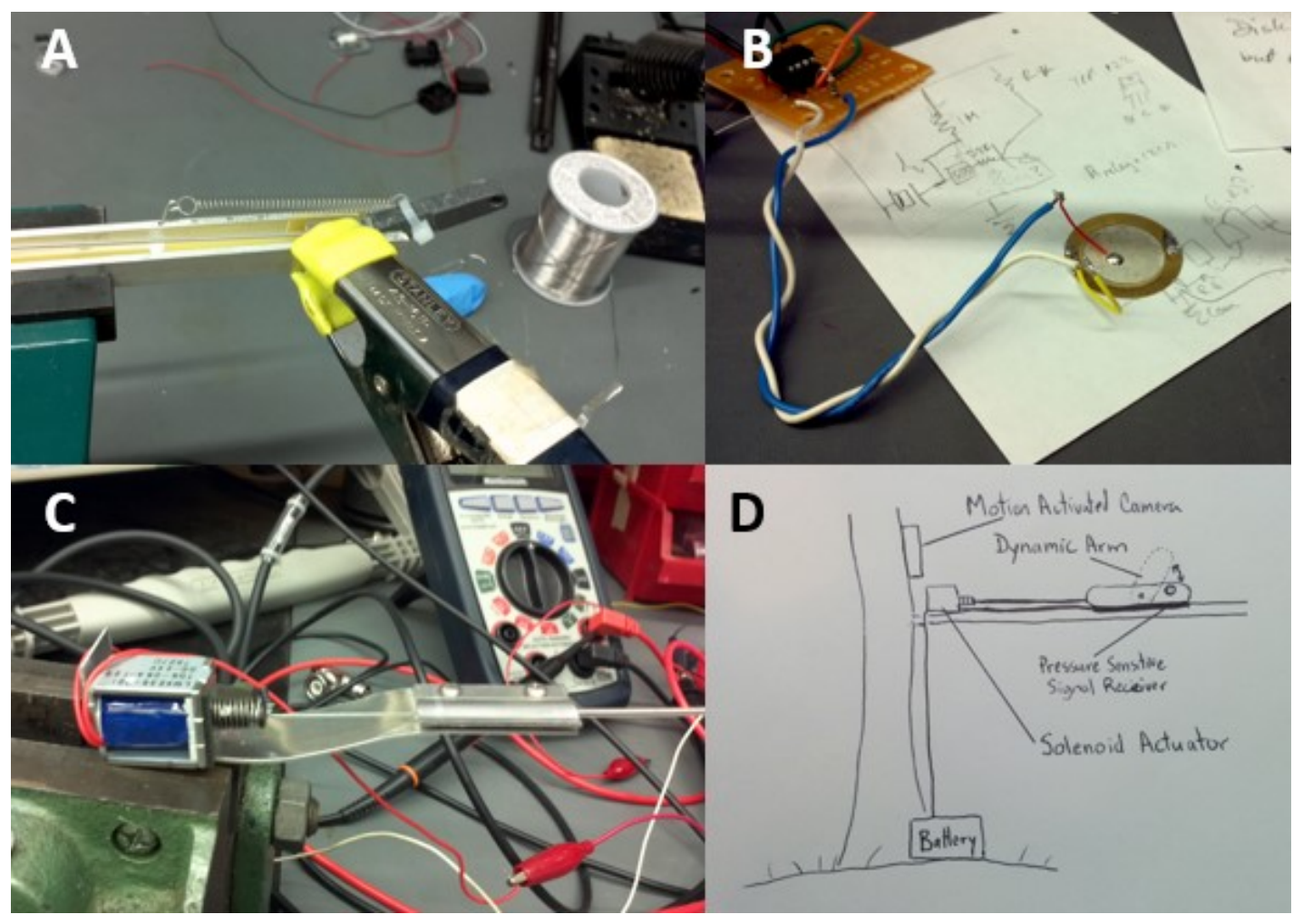

Figure 7-2: Photos from the prototyping process for a caterpillar model that could move upon attack by wild birds. A) The dynamic arm with spring-loaded release motion. B) A pressure sensitive signal receiver which would be triggered by a bird peck. C) A solenoid actuator which would activate and reset the dynamic arm. D) A schematic of how the model would be deployed in the field. 
Outside of anecdotal reports and the chick experiment in Chapter 6, we know relatively little about how birds react to caterpillars with mimetic traits. Future field experiments with wild birds should consider using motion-activated cameras (e.g., BirdCam ${ }^{\circledR}$ by Wingscapes ${ }^{\circledR}$ ) to record the behaviour of wild birds responding to either live or model caterpillars with and without mimetic features like eyespots. Such recordings would inform our understanding of the behavioural processes involved. One approach might be to deploy models with various combinations of the phenotypes (i.e., eyespots, posture, size, sinuous movements), then monitoring these models with birdoptimized trail cameras. I did some preliminary work with trail cameras optimized for mammals in 2011 and found that they were ineffective at reliably monitoring bird predation on pastry caterpillars. More generally, experiments with model prey should consider employing motion-activated camera systems on a subset of their models to gain improved insight into the specific predator community involved and how they interact with the models.

\subsubsection{Mimetic fidelity, body size, and behavioural mimicry}

As mentioned in the discussion section of chapter 6, recent work with hover flies has observed that mimetic fidelity is positively correlated with body size (Penney et al. 2012). Using the techniques developed in chapter 4 it should be possible to quantify mimetic fidelity across an entire taxonomic group, like the Papilio genus of Papilionidae or the Macroglossinae subfamily of Sphingidae. With a continuous measure of fidelity, body size data, and a robust phylogeny it becomes possible to test the fidelity-size relationship in this system. Currently, there is a lack of suitable photos for this endeavor 
(see photo requirements outlined in Chapter 4), but with long term photographic databases (e.g., Janzen and Hallwachs 2014), combined with social media websites like Flickr, this project may be possible in the near future. I set up a Flickr group called "Caterpillars with Eyespots" (https://www.flickr.com/groups/eyespots/) to collect images, partly with the hope that they could one day be used in such endeavours. Note that photographs from Flickr have previously provided useful data for scientific publications (e.g., Winterton et al. 2012; Nyffeler \& Knörnschild 2013). It may also be possible to estimate mimetic fidelity using human rankings, however it is unclear how well such rankings would correlate with those of ecologically relevant predators.

Work from the hoverfly system has revealed that that good mimics also tend to be behavioural mimics (Penney et al. 2014). Anecdotally, it does seem to be that the best snake mimics also have the most intricate displays, as evidenced by Hemeroplanes ornatus (Sphingidae), Madoryx plutonius (Sphingidae), and Phyllodes imperialis (Noctuidae). Investigating the relationship between mimetic fidelity and behavioural mimicry in this system is challenging. Specifically, it requires behavioural data gleaned from natural history observations that are inconsistently reported and subjective, or hardwon through numerous behaviour tests (as they were in Penney et al. 2014). It then requires some means of quantifying several disparate behaviours into a single comparable and quantitative metric. If video from standardized behaviour tests were to be collected into a single searchable database it would facilitate research into this and other questions in the behavioural ecology of anti-predator defence in caterpillars. 


\subsubsection{Evolutionary consequences of deception: speciation}

Many caterpillars with eyespots come from species rich clades; representative examples include the genera Papilio (Papilionidae) and Xylophanes (Sphingidae). This has led me to wonder whether the evolution of an anti-predator trait might influence the subsequent rate of diversification in that lineage. Botanists have argued that pollinator deception has facilitated the species diversity in orchids (Cozzolino \& Widmer 2005; Xu et al. 2012), but could predator deception work in a similar way? Generally speaking, one might predict that lineages which possess a particularly effective anti-predator trait would have greater rates of divergence. We can make the more specific prediction, however, that prey with an anti-predator strategy that involves predator deception (e.g., masquerade and mimicry) will have greater rates of diversification than prey using strategies that are more host-plant (or background environment) dependent (e.g., crypsis and aposematism).

At least within Lepidoptera the efficacy of both crypsis and aposematism rely on the caterpillar inhabiting its specific host plant; cryptic species because they depend on matching the background of their host plant to avoid detection, and aposematic species because their chemical protection is derived from diet-sourced chemicals present in their host plant (though the initial evolution of aposematism itself seems to be related to the signal environment; Prudic et al. 2007). For those caterpillars employing deception (i.e., masquerade or mimicry) their defence should prevent or deter attack even in novel habitats. Thus, in the rare event that an adult female lays her eggs on a novel host plant, prey whose defences are host-plant dependent may experience a greater increase in predation pressure compared to prey who deceive their predators post-detection. If this is indeed the case, those species whose defence is less context-specific may have a greater 
chance of colonizing new environments - a first step towards speciation. This idea is eminently testable, and the defensive strategies in larval lepidopterans offers a great system to examine this question.

A first step towards testing this idea would be to quantify variation in host plant use among species using distinct anti-predator defences. One would predict that species employing crypsis and aposematism would have lower diversity in their host plants than species which employ masquerade or mimicry. Indeed, this is precisely what Higginson et al. (2012) found - masquerading species fed on a greater diversity of host plants (measured by number of host plant genera or families) when compared to cryptic species. Whether mimetic species, like caterpillars with eyespots, also have a greater diversity of host plants and whether the exploitation of greater diversity in host plants translates to greater rates of diversification remains untested. Ancestral character reconstructions would also need to show that increased diversity of host plant is a consequence of evolving deception, as opposed to deception evolving in response to diversity in host plant assemblage - though either result would be equally interesting.

Phylogenetic tools now exist which can assess whether certain traits (e.g., defence strategy, diversity of host plants) are associated with an increase or decrease in diversification. One important limitation however is that many of the large molecular phylogenies that would be useful for this kind of analysis are built using diversified sampling, a technique where species are chosen to represent numerous subgroups (i.e., to maximize diversity). Such phylogenies are poor representations of the true diversity in each of the subgroups, and can lead to significant bias if used to estimate non-constant diversification rates (Höhna 2014). This problem is compounded by the fact that the 
many of the readily available phylogenies only include extant species. Papilionidae and Macroglossinae (Sphingidae) are two well-studied groups that seem suitable for such diversification analyses. In fact Papilionidae have already been used to examine the processes involved in generating the latitudinal gradients in species diversity (Condamine et al. 2012). Dr. Condamine was enthusiastic about possibly examining the effect of larval phenotype on diversification rate when I contacted him, and I am currently working with Dr. Akito Kawahara and Dr. Jesse Breinholt to build a more representative phylogeny for Macroglossinae that would be suitable for diversification analysis. If indeed the defensive strategy can constrain or facilitate the species radiation it would reveal an unappreciated evolutionary consequence resulting from anti-predator adaptation.

An underlying assumption of the hypothesis outlined above is that predation severely limits the radiation of species to novel host plants. This could be tested using a pastry caterpillar experiment. For simplicity, one could begin by testing whether eyespots facilitate radiation. Treatments could include: colour-matched (well-camouflaged), colour-mismatched (poorly camouflaged), colour-matched with eyespots, and colourmismatched with eyespots. All 4 treatments would be deployed on two tree species, one tree that treatments (i) and (iii) are calibrated to spectrally match (based on a bird vision model), and another tree that treatments (ii) and (iv) are calibrated to match. Thus each tree would have 2 colour-matched and 2 colour-mismatched treatments, but allows the researcher to disentangle the effect of the specific colour used from the more general question of colour matching. The prediction here is that the survival cost of being colourmismatched is greater for prey lacking eyespots. It would be possible to do a similar 
experiment with masquerade (e.g., bird-dropping mimic), but the 'masquerade' treatment would be identical between tree species. Also, attempts would have to be made to spectrally calibrate the masquerade models to match real bird-droppings.

\subsubsection{Innate fear in the evolution of mimicry}

Innate fear is well documented in birds, mammals, and fish (e.g., Pough 1988; Hawkins et al. 2004; Caro 2005), yet role of innate processes in the evolution of mimicry has largely gone unstudied. This is problematic as innate aversion seems to have been the primary force that led to the evolution of mimicry in many cases. Both Pough (1988) and Janzen et al (2010) make a strong case for innate processes driving the mimicry of snakes and predator eyes in caterpillars. Innate aversion by the predator seems to have driven the evolution of mimicry in other systems as well including mimicry of venomous snakes by non-venomous ones (reviewed by Pough 1988), and the mimicry of ants by spiders (Nelson \& Jackson 2006; Nelson et al. 2006). Despite this growing body of evidence that innate fear or aversion to noxious co-occurring animals, the fact that these aversions might play a key role in generating or maintaining mimicry does not seem to be widely acknowledged or well understood. Instead this potentially important and general phenomenon exists largely as a collection of disparate facts and observations from various systems. A comprehensive review and theoretical treatment of this topic would be timely.

Some emerging questions about the role of innate processes in mimicry include: What features of model are programmed into these innate 'archetypes'? Why those features and not others? To what extent can these innate aversions be altered over the 
lifetime on an animal though experience and learning? How is innate fear related to the processes of neophobia and dietary conservatism? For example, does neophobia facilitate the evolution of mimicry by allowing poor mimics to arise, or does weaken selection for mimicry by failing to provide sufficient adaptive value to mimetic phenotypes over nonmimetic polymorphisms? Clearly, the role of innate processes in the evolution of mimicry remains an area wide open for future work. 


\section{Appendices}

\section{Appendix A Blog posts from “Caterpillar Eyespots” highlighting Moss' descriptions of snake-mimic caterpillars}

\section{A.1 Moss' account of Hemeroplanes larvae in Para}

When you are starting to dig through the literature for a new research topic, it is often very rewarding to go back and actually read the earliest accounts you can find - especially for biologists. This is because early naturalists wrote beautifully and often provide very detailed, thorough descriptions. Sometimes you are even rewarded with really amazing engravings, paintings, or sketches of the specimens they observed or collected. I also feel that going back to these early works gives you a great historical perspective on the work you are doing. Occasionally you even feel a kind of connection to these writers when you are studying the same phenomena or specimens they witnessed (or collected) 50-100 years ago (perhaps even earlier). Many biologists have this experience when they read Darwin's On the Origin of Species. If not, there are many other places to look.

Some really detailed work on South American hawkmoths, and their larvae, was done by a man named Rev. Arthur Miles Moss, M.A., F.Z.S., F.E.S., British Chaplain of Pará. His work is interesting to me because of his detailed descriptions of many caterpillars with eyespots, and their close relatives. I haven't been able to find much information on the man himself except for what is posted on the British Natural History Museum website which I have reproduced below:

Arthur Miles-Moss (1873-1948)

Born in Liverpool, Arthur Miles-Moss studied at Trinity College, Cambridge. He was ordained deacon in the Church of England in 1895 and then priest the following year. In 1897 he travelled to South America and from 1907 to 1910 he lived in Peru before moving to Pará in Brazil, 100 miles south of the mouth of the river Amazon where he stayed until 1945. In 1912 he built the Pará Anglican Church. His parish covered a vast area of Brazil and he was known as the vicar of the largest parish in the world. In his spare time he began a detailed study of the insects of the region and amassed a large collection of butterflies and moths (Lepidoptera). He also reared and made detailed drawings of the caterpillars and pupae. A talented artist, he also painted many land-and seascapes, some of which he exhibited at the Royal Academy, London.

Moss put his skills as an accomplished artist to work painting the countless caterpillars and pupae 
that he reared in his spare time. The Natural History Museum has a collection of his manuscripts, drawings and photographs. Many of these are watercolour paintings or drawings of caterpillars annotated with various observations he made when rearing the larvae. Their collection also hold $\sim 25000$ preserved specimens from the caterpillars that he reared. Here is a sample of his artwork:

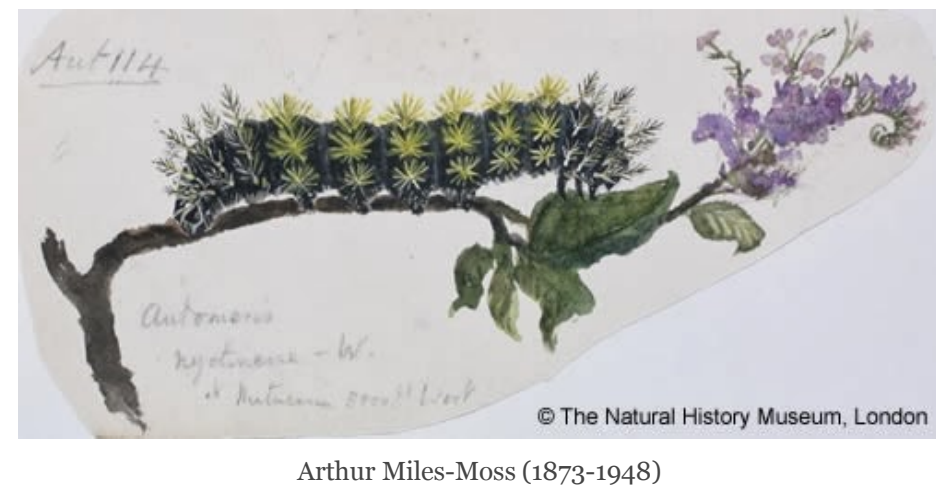

Drawing of the larval stage of the Saturniid moth (Leucanella nyctimene)

Watercolour on paper, $78 \times 165 \mathrm{~mm}$.

I would love to visit this collection. I have seen a few other pieces of his work from the parts of it that he published, but I'm sure that there are still many of his paintings and detailed notes that for now remain out of sight. The two notable publications I have been interested in are "On the Sphingidae of Peru" from 1912 and "Sphingidae of Para, Brazil" written in 1920. They have detailed descriptions of many hawkmoth caterpillars - some of which have eyespots - and include notes on their behaviour.

I have written before about the extraordinary snake-mimic caterpillars of Hemeroplanes $s p$. here. Moss also collected and wrote about this species. To give you an idea of how spectacular these caterpillars can be here is a photo:

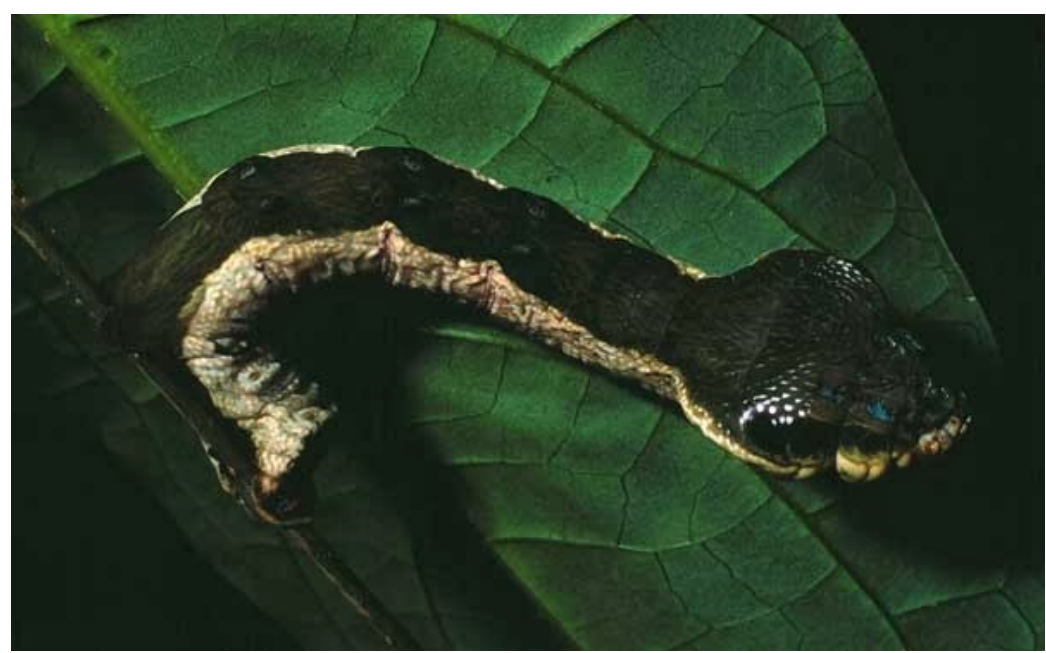

Hemeroplanes triptolemus (Sphingidae) Photo of specimen 87-SRNP-1156 from the Janzen and Hallwachs database. 
Moss (1920) gives a really nice description of this caterpillar (Moss refers to it as Leucorhampha triptolemus) and includes notes on its intricate defensive behaviour. I have reproduced his description of the mimetic and behavioural features below and added photos of a live specimen to illustrate the features he is describing:

\section{Leucorhampha triptolemus. (Plates 6 \& 9.) [p. 391-392]}

The description which I now quote was written in November 1916 for the larva of ornatus, but as it applies equally well for triptolemus and must be regarded as doing double duty, I prefer to insert it here. The larva is quite one of the most remarkable of living creatures that I have ever seen, a perfect Aaron's rod, combining in the most novel and striking way the principles of protective resemblance with an aggressive snakemimicry. When at rest as an adult caterpillar, it hangs by two pairs of claspers in the vertical from the stem of its food-plant, and appears to be nothing but a broken branch covered with a creamy white lichen. A strange black chequered dorsal design, with a gradual intensification of the grey on certain segments completes the deception.

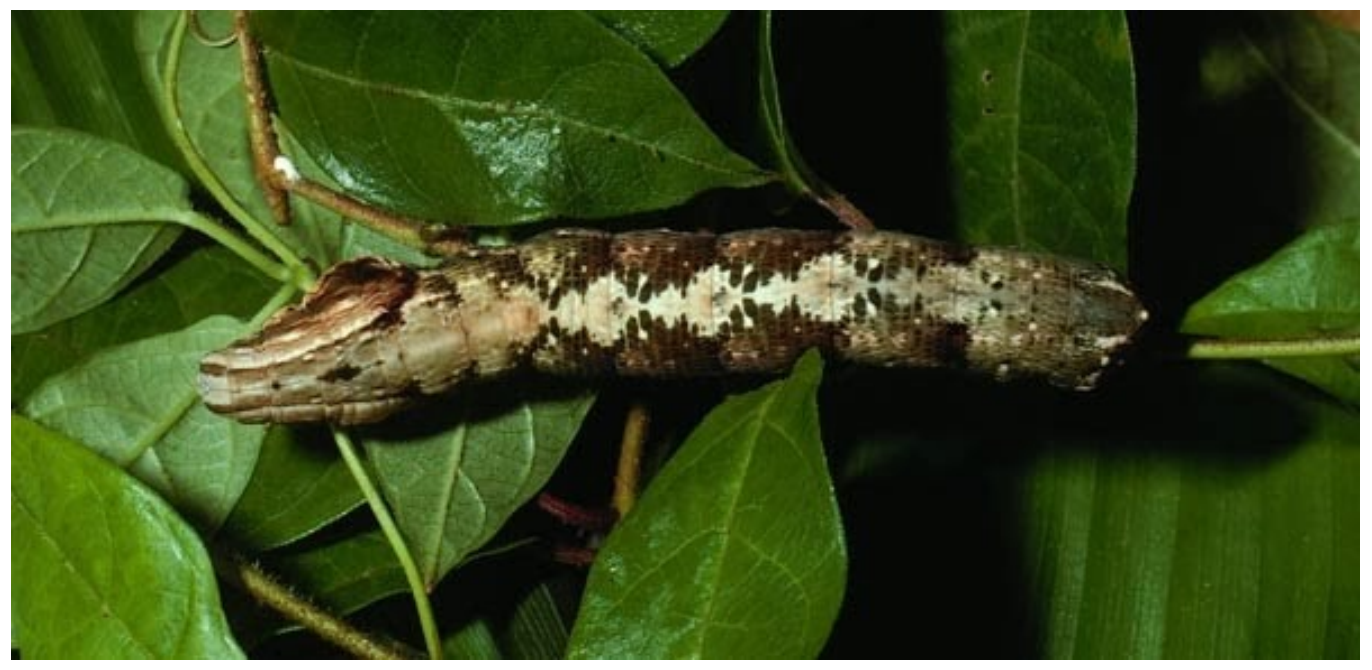

Hemeroplanes triptolemus (Sphingidae)

Photo of specimen 03-SRNP-11366 from the Janzen and Hallwachs database. 
The wonder, however, is if possible exceeded when, on being disturbed, this marvel of creative evolution endeavours once more to deceive by turning into a snake, and in quite a different way to that adopted by Xylophanesor even by its fairly close relative Madoryx pluto.

Aside: The relationship between Madoryx and Hemeroplanes is still being worked out. It looks as though they are indeed related but evolved snake mimicry independently - that is to say that it is unlikely that both species mimic snakes simply because they share a common snake-mimicking ancestor.

Though this wonderful transformation wants to be seen in life to be fully appreciated, I may explain briefly that the effect is produced by the creature turning itself over and exhibiting its ventral area, which is adorned by a broad band of dark olive-green with the three anterior sets of claspers completely withdrawn and scarcely visible.

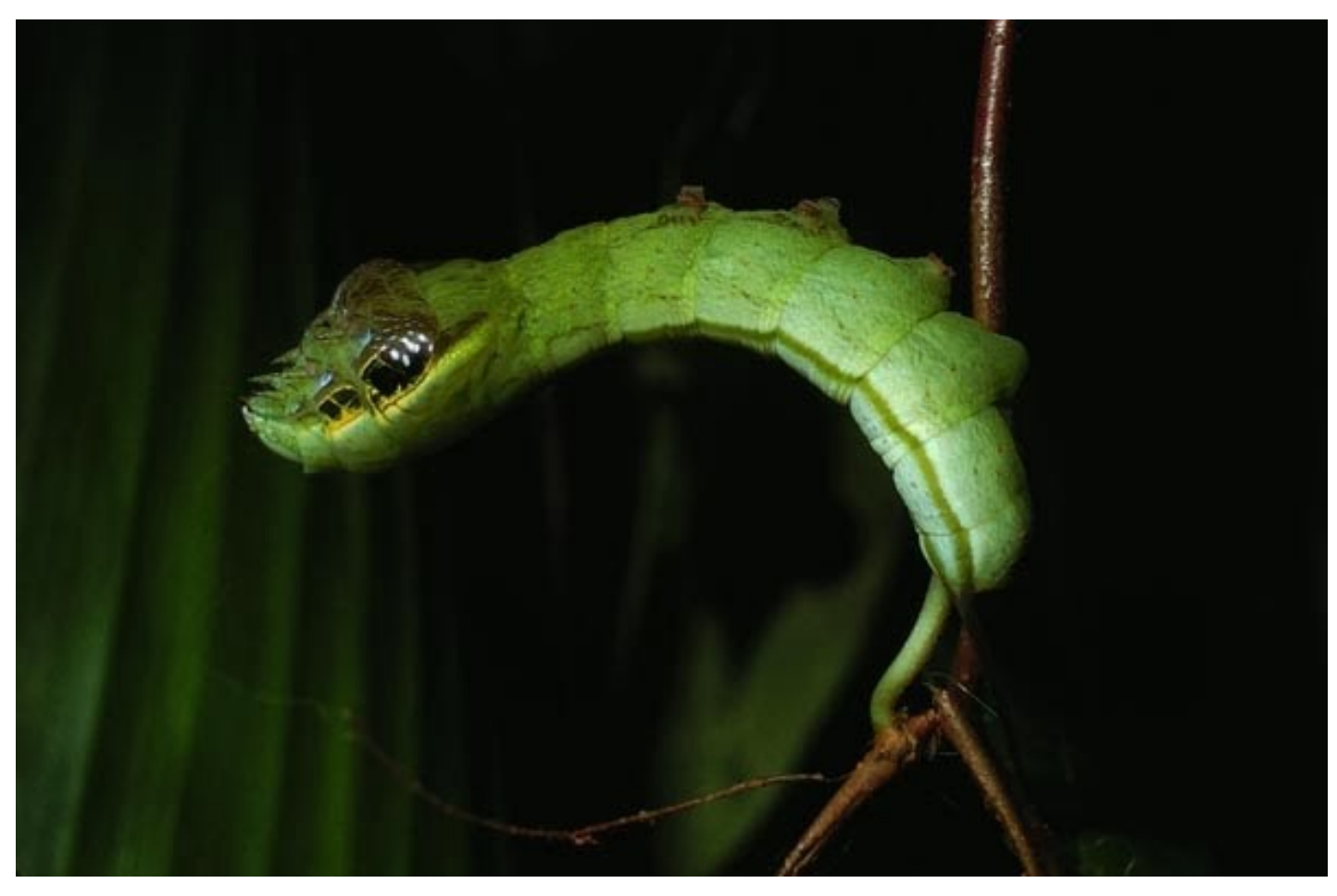

Hemeroplanes triptolemus (Sphingidae)

Photo of specimen 03-SRNP-11366 from the Janzen and Hallwachs database. 
The thoracic segments, which are always swollen, become puffed out laterally to an exaggerated extent; $a$ pair of black eyes on segment 4 ,

hitherto concealed and

situated behind the now recumbent and wholly inconspicuous legs, open out;

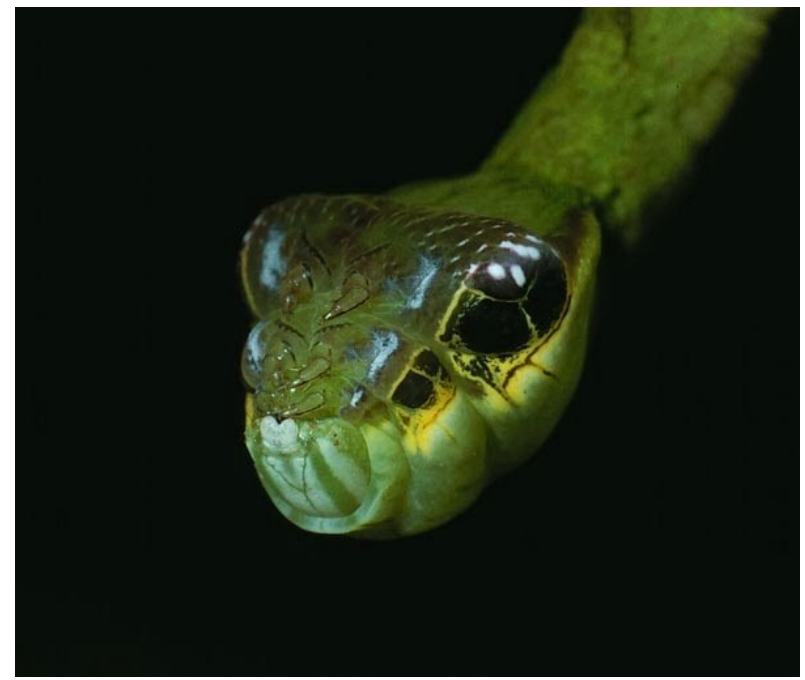

Hemeroplanes triptolemus (Sphingidae) Photo of specimen 03-SRNP-11366 from the Janzen and Hallwachs database.

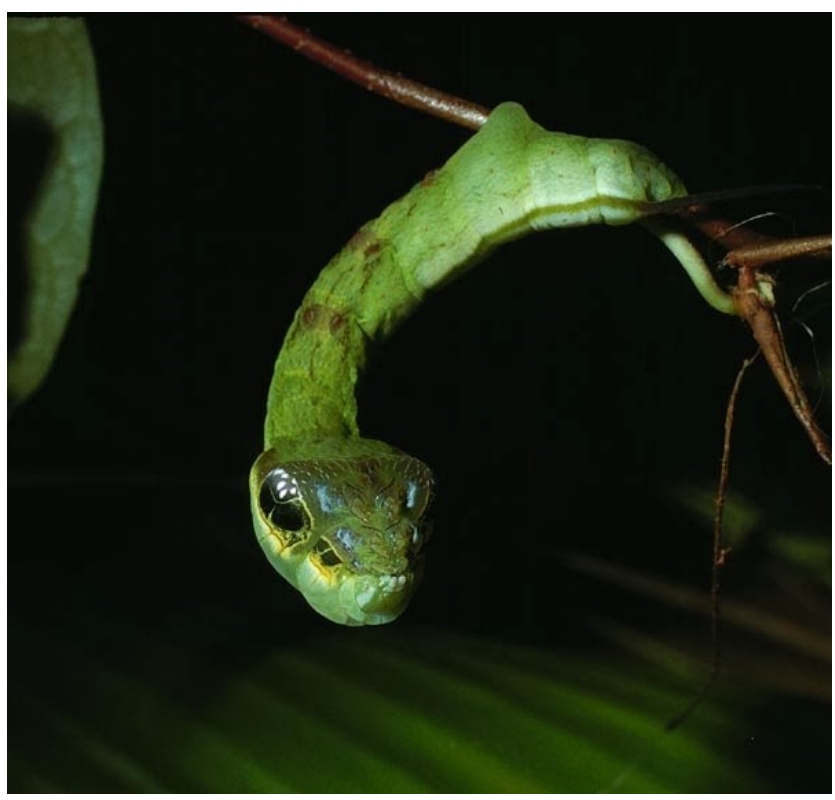

the cheeks appear to be adorned by yellow scales with black edges; and the fraudulent notion that one is beholding merely the head and neck of a formidable, if small, snake is carried to a nicety by the rigidity of the curve adopted.

Hemeroplanes triptolemus (Sphingidae)

Photo of specimen 03-SRNP-11366 from the Janzen and Hallwachs database. 
Then, as if to mesmerize, a swaying side-to-side motion is kept up for an appreciable number of seconds, before the creature, seeming to realize that an attack is no further contemplated, gradually closes its false eyes and relapses once more into diurnal slumbers. That this mimicry of the fore-part of a small serpent, if mimicry it be, for it is hard to give it any other name, should be chiefly produced on the ventral surface, a feature peculiar in itself, and that every detail should so contribute in perfecting the deception, is altogether remarkable.

Blog post published by Thomas Hossie - 14 December 2013 at: http://caterpillar-eyespots.blogspot.ca/2013/12/moss-account-of-hemerplanes-larvaein.html

\section{A.2 Moss' account of another snake-mimic: Madoryx plutonius}

In my last post, I shared Moss' description of snake-mimicry in Hemeroplanes triptolemus caterpillars. In the same publication - The Sphingidae of Para, Brazil - he describes another spectacular caterpillar he encountered - Madoryx plutonius (he refers to it at M. pluto). This is another sphingid which also appears to mimic a snake. Moss supposed that these two species were close relatives, and this is now supported with molecular genetics as well (Kawahara et al 2009). Interestingly though, despite being related species the colour patterns and defensive behaviours in each species are so distinct that I suspect that they evolved independently. One of my current projects involves identifying how many times eyespots and snake mimicry have evolved independently in Sphingidae.

For now, I'd just like to showcase this amazing caterpillar and Moss' description of its defensive behaviour (see below). These photos of Madoryx plutonius are again taken from the Janzen and Hallwachs database. The specimens were collected in Costa Rica as part of the ongoing inventory project within the Área de Conservación Guanacaste run by Dan Janzen and Winnie Hallwachs. 


\section{Madoryx pluto. (Plate 6.) [p. 394]}

The larva in its final in star, if the championship among frauds be already won by the Leucorhawpha species [Moss is referring to H. triptolemus], nevertheless runs them closely, and takes a very high second place in the perfect success of those devices resorted to in first simulating the living stalk or stem of its food-plant, and then by endeavouring to terrorize one into the belief that one is gazing at an open-mouthed snake with red jaws and a couple of fierce, blue- black eyes' set immediately above them.

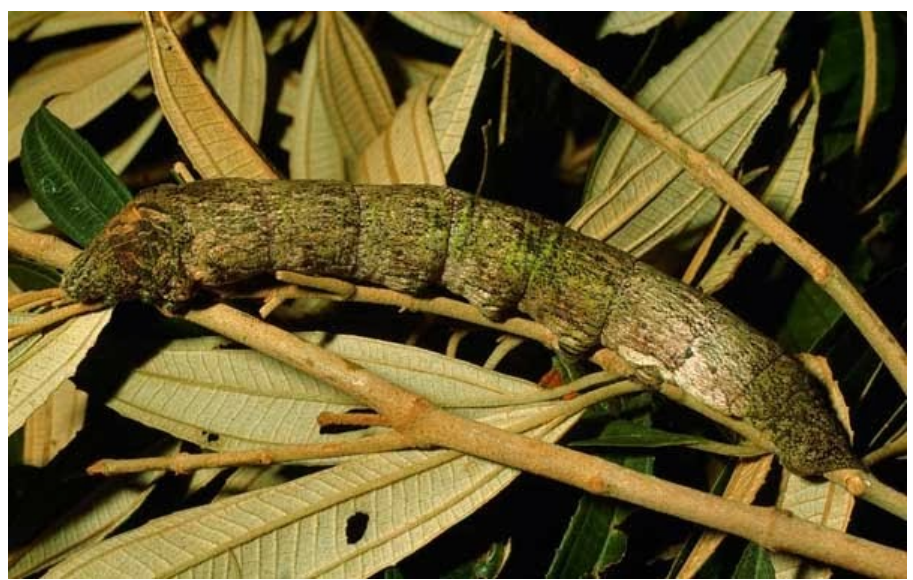

Final instar Madoryx plutonius caterpillar (at rest)

Photo of specimen 95-SRNP-4624 from Janzen and Hallwachs database.

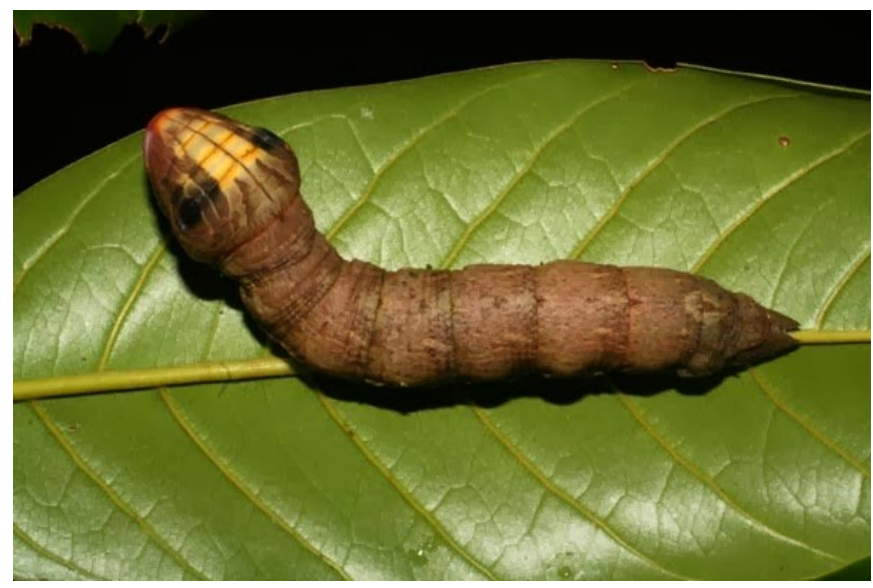

Final instar Madoryx plutonius caterpillar (defensive posture)

Photo of specimen 95-SRNP-4624 from Janzen and Hallwachs database.

Final instar Madoryx plutonius caterpillar (defensive posture)

Photo of specimen 95-SRNP-4624 from Janzen and Hallwachs database. 
This particular effect is produced entirely on the dorsal area by the larva lowering its head towards its legs, puffing out its thoracic segments (not laterally this time), and exhibiting these wonderfully brilliant touches of external ornament which at all other times when the caterpillar is sleeping or even when it is eating or in motion are entirely concealed within the interstices of its segments, and looks a perfect stick of a light brown coloration. Though I secured two photos, to take its portrait as a snake in pencil and paint was an exceedingly difficult task, for it would never maintain the attitude for a sufficient length of time, and soon got tired of even repeating the hoax for the edification of my much-impressed friends.
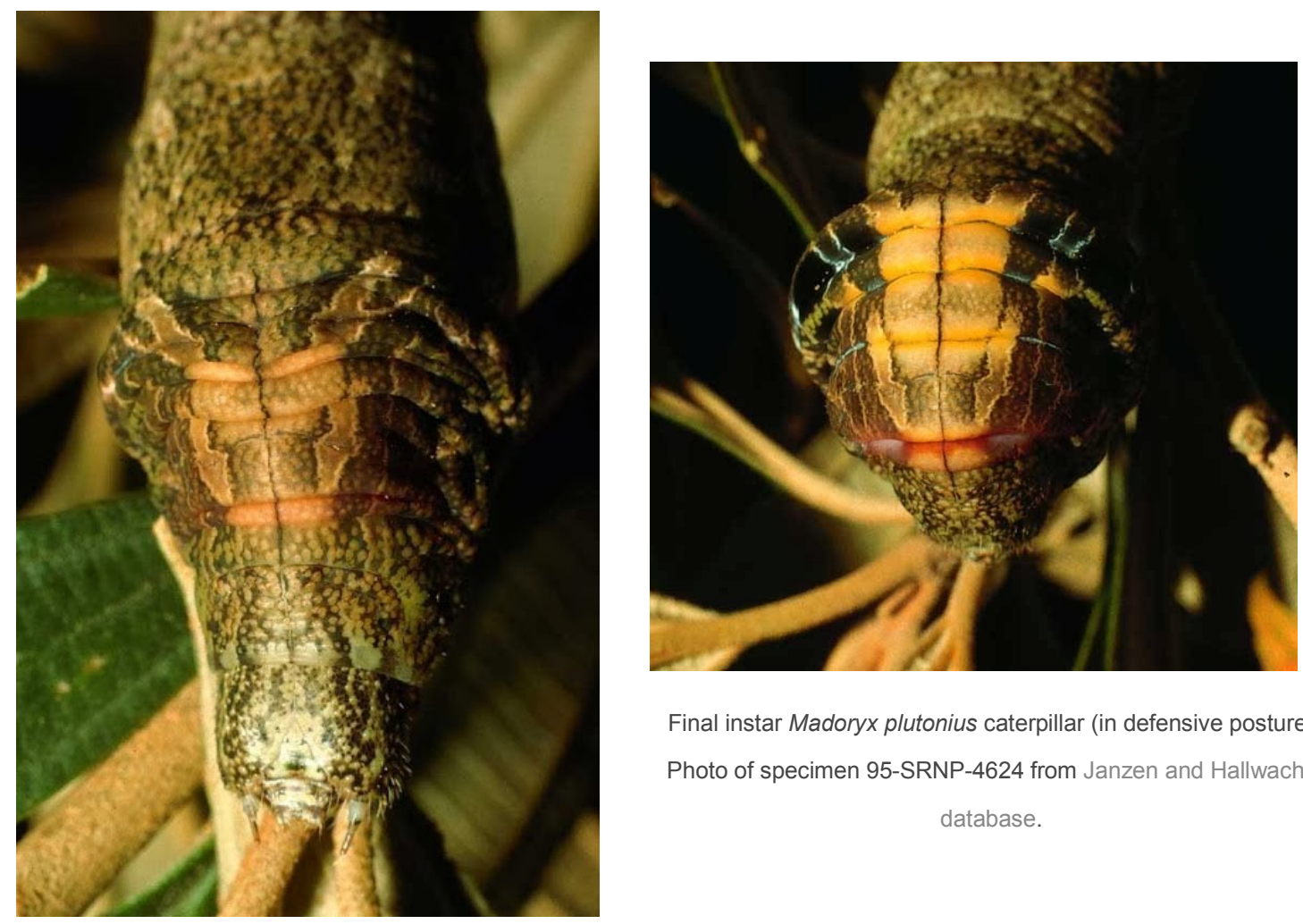

Final instar Madoryx plutonius caterpillar (in defensive posture) Photo of specimen 95-SRNP-4624 from Janzen and Hallwachs database.

Final instar Madoryx plutonius caterpillar (at rest)

Photo of specimen 95-SRNP-4624 from Janzen and Hallwachs database. 

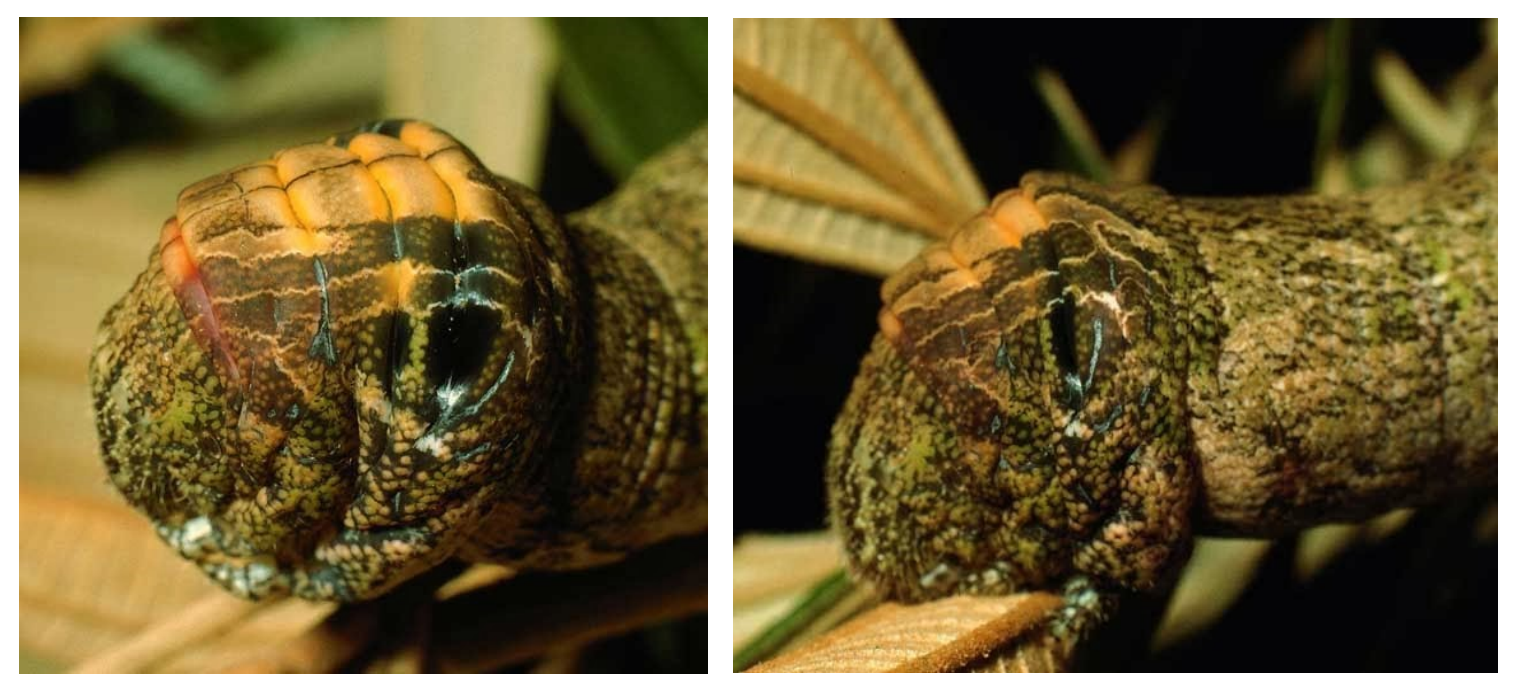

Final instar Madoryx plutonius caterpillar

Final instar Madoryx plutonius caterpillar

(in defensive posture)

Photo of specimen 95-SRNP-4624

from Janzen and Hallwachs database.

(recovering to rest posture)

Photo of specimen 95-SRNP-4624

from Janzen and Hallwachs database.

Blog post published by Thomas Hossie - 23 December 2013 at:

http://caterpillar-eyespots.blogspot.ca/2013/12/moss-account-of-another-snake-

$\underline{\text { mimic.html }}$ 


\section{Appendix B Supplementary material for Chapter 2}

Table S2-1: Insectivorous birds observed in each location during the associated field trail conducted over the summer of 2010 near Kemptville

(Sites 1 and 2) and Oxford Mills (Site 3), ON, Canada

\begin{tabular}{|c|c|c|c|c|c|c|c|c|c|c|}
\hline \multirow[t]{2}{*}{ Bird species } & \multirow[t]{2}{*}{ Migration } & \multicolumn{3}{|l|}{ Site 1} & \multicolumn{3}{|l|}{ Site 3} & \multicolumn{3}{|l|}{ Site 3} \\
\hline & & 25 May & $14 \mathrm{Jul}$ & 11 Aug & 8 Jun & $20 \mathrm{Jul}$ & 18 Aug & $7 \mathrm{Jul}$ & $30 \mathrm{Jul}$ & 24 Aug \\
\hline Cardinalis & Resident & & & & & & & & & $\mathrm{x}$ \\
\hline Poecile atricapilla & Resident & & $\mathrm{x}$ & $\mathrm{x}$ & $\mathrm{x}$ & $\mathrm{x}$ & $\mathrm{x}$ & & $\mathrm{x}$ & $\mathrm{x}$ \\
\hline Sitta carolinensis & Resident & & & $\mathrm{x}$ & & & & & & \\
\hline Spinus tristis & Resident & $\mathrm{x}$ & $\mathrm{x}$ & & & $\mathrm{x}$ & & & $\mathrm{x}$ & \\
\hline Agelaius phoeniceus & Short-Medium & & & & $\mathrm{x}$ & & & & & \\
\hline Bombycilla cedrorum & Short-Medium & & & & & & & & & $\mathrm{x}$ \\
\hline Passerculus sandwichensis & Short-Medium & & & & & $\mathrm{x}$ & & & $\mathrm{x}$ & \\
\hline Quiscalus quiscula & Short-Medium & & & & $\mathrm{x}$ & & & $\mathrm{x}$ & & \\
\hline Sayornis phoebe & Short-Medium & & & & $\mathrm{x}$ & & & & $\mathrm{x}$ & \\
\hline Spizella passerina & Short-Medium & $\mathrm{x}$ & & & $\mathrm{x}$ & & & $\mathrm{x}$ & & \\
\hline Turdus migratorius & Short-Medium & & $\mathrm{x}$ & & $\mathrm{x}$ & $\mathrm{x}$ & $\mathrm{x}$ & $\mathrm{x}$ & & $\mathrm{x}$ \\
\hline Vireo gilvus & Short-Medium & & & & & & & $\mathrm{x}$ & & \\
\hline Zonotrichia albicollis & Short-Medium & & $\mathrm{x}$ & $\mathrm{x}$ & & & & $\mathrm{x}$ & & $\mathrm{x}$ \\
\hline Dendroica pensylvanica & Neotropical & & & & & & & $\mathrm{x}$ & & \\
\hline Dendroica petechia & Neotropical & $\mathrm{x}$ & & & & & & & & \\
\hline Dumetella carolinensis & Neotropical & & & & & & & $\mathrm{x}$ & & \\
\hline Geothlypis trichas & Neotropical & $\mathrm{x}$ & $\mathrm{x}$ & $\mathrm{x}$ & & & & & $\mathrm{x}$ & $\mathrm{x}$ \\
\hline Mniotilta varia & Neotropical & & & & $\mathrm{x}$ & $\mathrm{x}$ & & & & \\
\hline Pheucticus ludovicianus & Neotropical & & & & & & & & $\mathrm{x}$ & \\
\hline Setophaga ruticilla & Neotropical & & & & & & & $\mathrm{x}$ & & \\
\hline
\end{tabular}




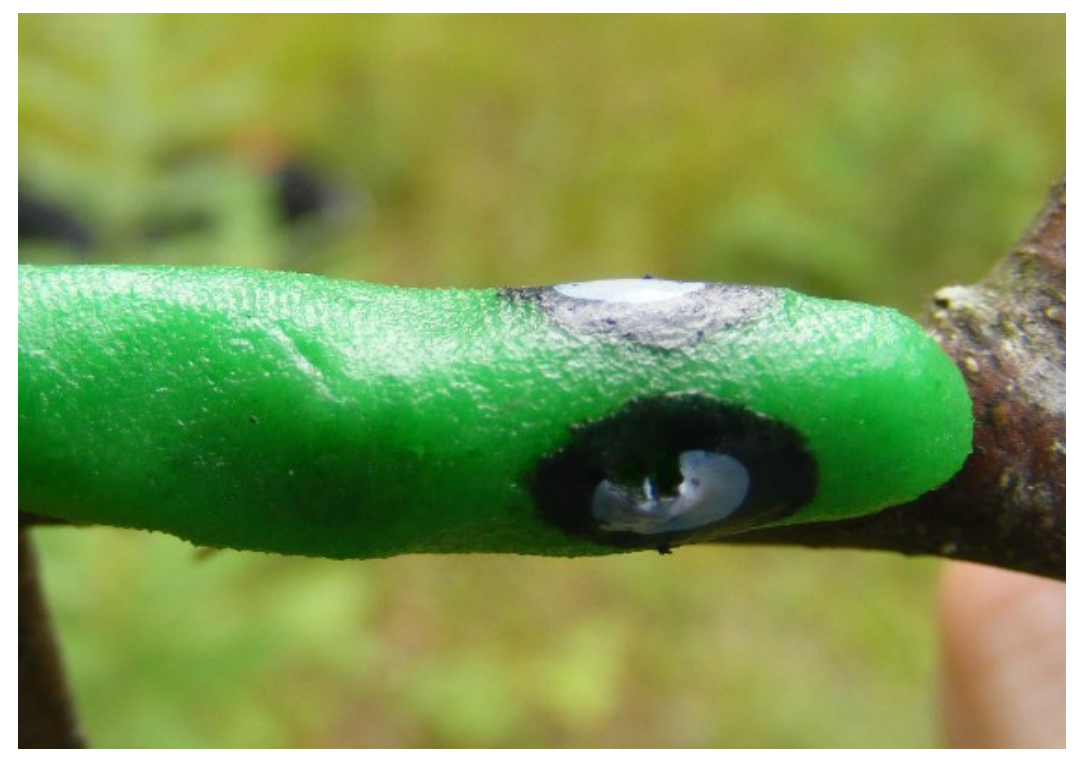

Figure S2-1 : Dorsolateral view of an eyespot pastry caterpillar used in our experiment.

Note that the pastry caterpillar in this photograph received a peck directly in the right eyespot. 
Table S2-2: Number of each tree species used to pin pastry caterpillars in each location at each site

\begin{tabular}{llllll}
\hline Site & Location & $\begin{array}{l}\text { Populus } \\
\text { tremuloides }\end{array}$ & $\begin{array}{l}\text { Populus } \\
\text { deltoides }\end{array}$ & $\begin{array}{l}\text { Betula } \\
\text { papyrifera }\end{array}$ & $\begin{array}{l}\text { Acer } \\
\text { rubrum }\end{array}$ \\
\hline 1 & 1 & 1 & 23 & 0 & 0 \\
& 2 & 1 & 23 & 0 & 0 \\
& 3 & 11 & 0 & 3 & 10 \\
2 & 1 & 6 & 0 & 10 & 8 \\
& 2 & 12 & 0 & 12 & 0 \\
& 3 & 10 & 0 & 8 & 6 \\
3 & & & & & \\
& 1 & 19 & 0 & 5 & 0 \\
& 2 & 8 & 2 & 4 & 10 \\
& 3 & 12 & 0 & 0 & 12 \\
\hline
\end{tabular}



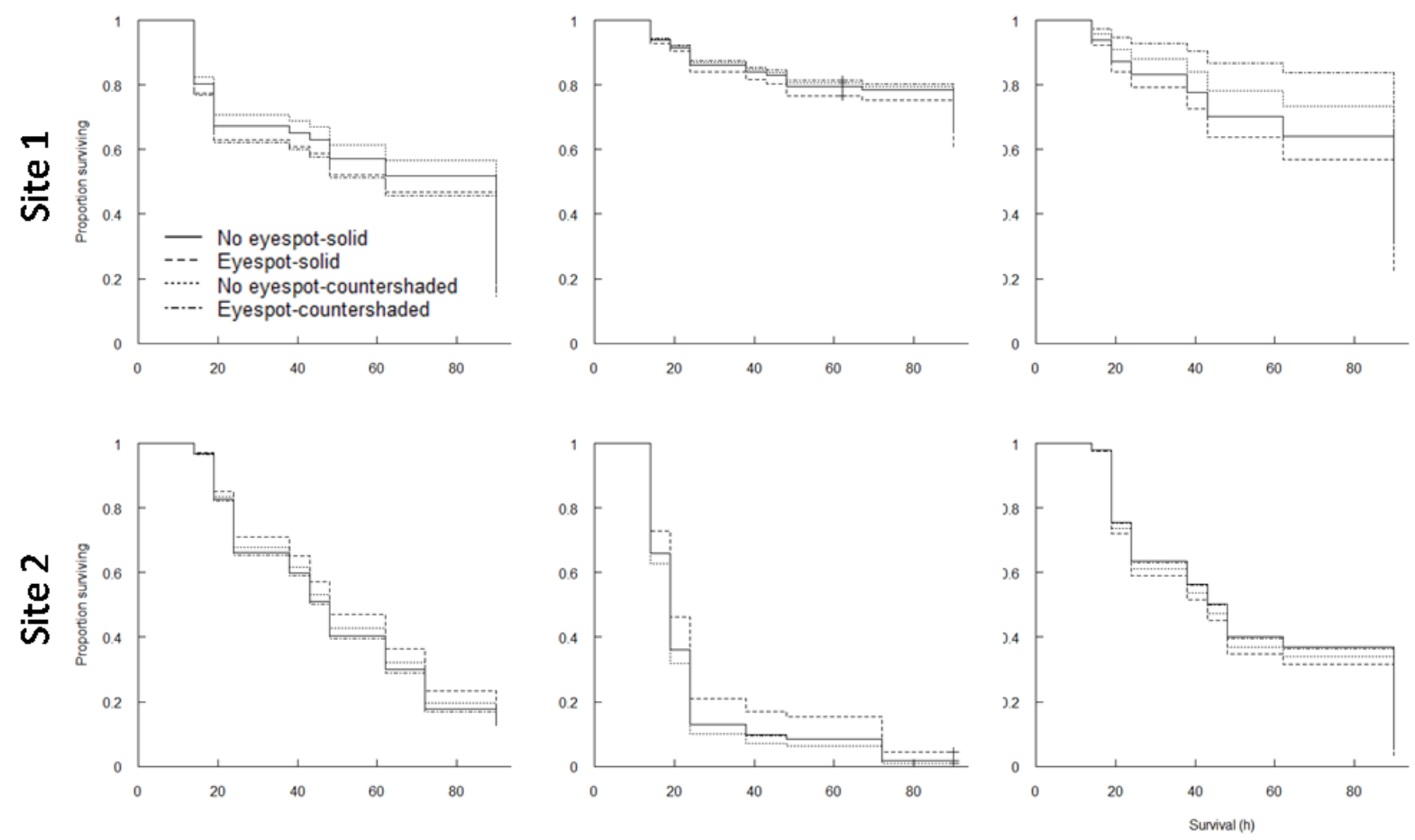

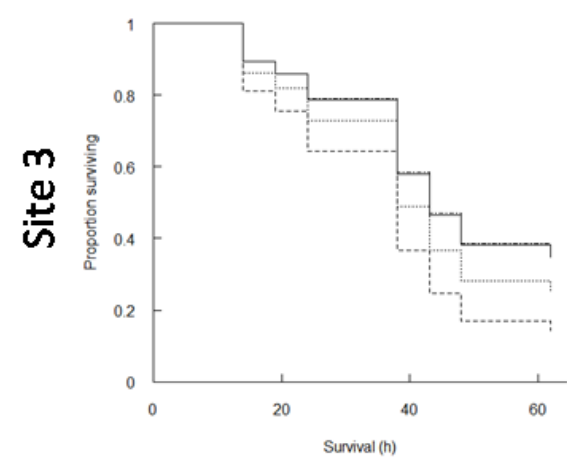

Location 1

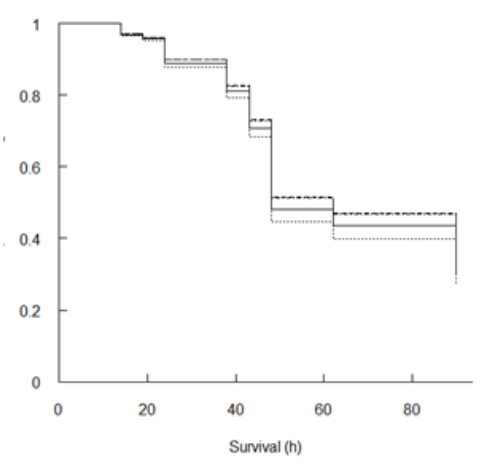

Location 2

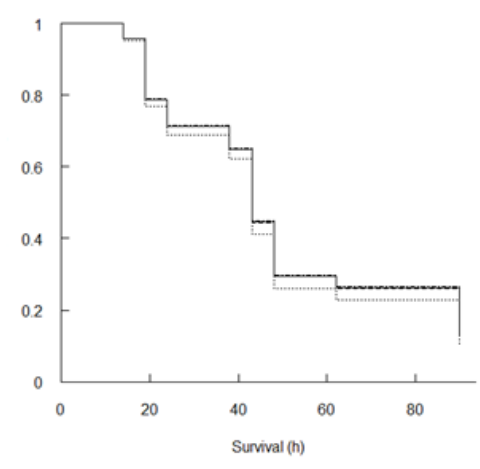

Location 3

Figure S2: Fitted cumulative survival charts comparing the survival of four treatments of pastry caterpillars in each of the nine locations. Note that each chart is based on $N=96$ baits and, for clarity, these survival curves are fitted without controlling for the tree species on which they were pinned. 

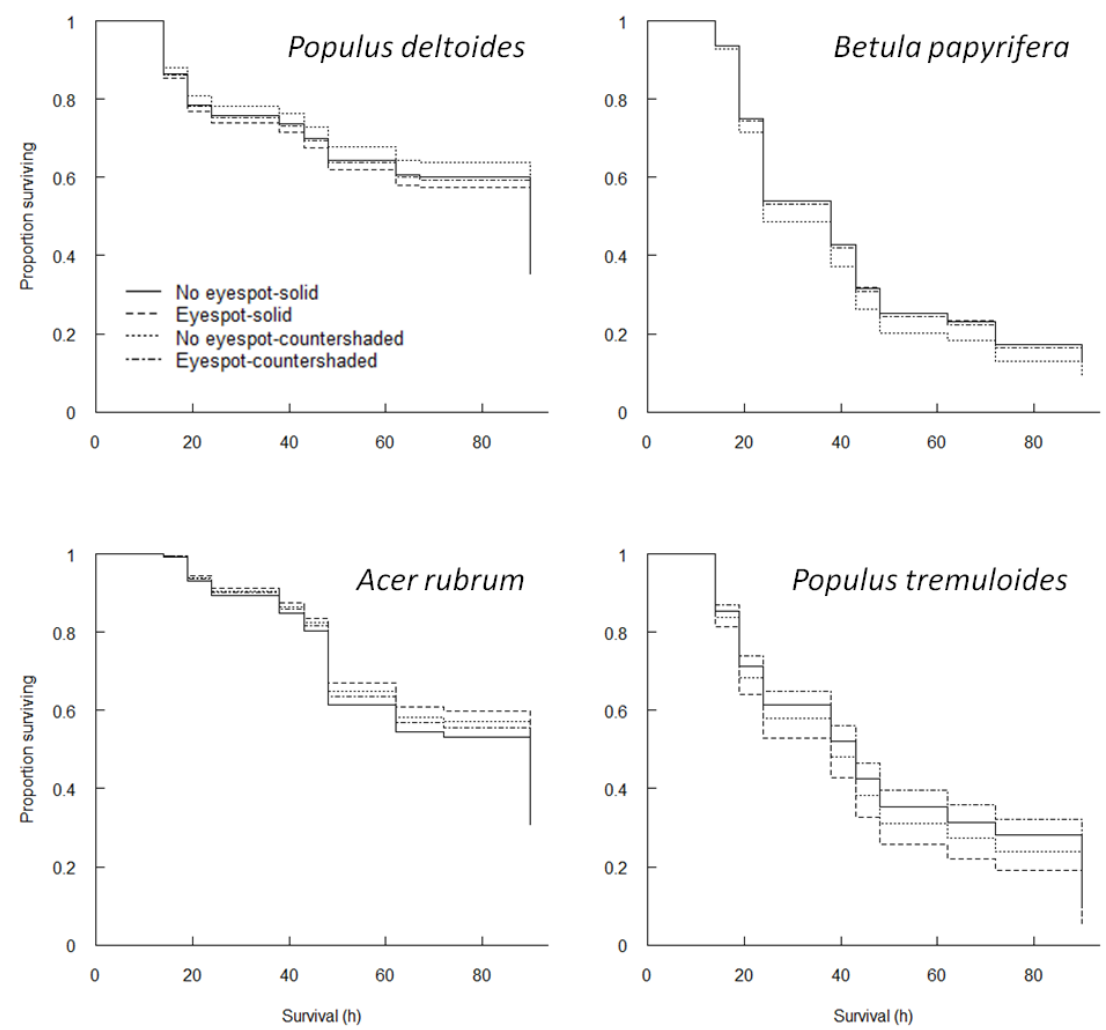

Figure S2-3: Fitted cumulative survival charts comparing the survival of four treatments of pastry caterpillars pinned to different tree species. Note that, for clarity, these survival curves are fitted without controlling for the location where the trials took place. Sample sizes also varied among the four charts: Populus tremuloides: $N=320$; Populus deltoids: $N=192 ;$ Betula papyrifera: $N=168$; Acer rubrum: $N=184$. Note: with the exception of $P$. tremuloides, there was no significant difference in the survival rate of caterpillars among the four eyespot/colour treatments $(P$. tremuloides: overall Wald $=14.67, d f=3, N=320, P=0.002 ; P$. deltiodes: overall Wald $=3.1, d f=3, N=192, P=0.38 ;$ B. papyrifera: overall Wald $=2.01, d f=3, N=$ 168, $P=0.57$; A. rubrum: overall Wald $=1.48, d f=3, N=184, P=0.69$ ). 
Table S2-3: Results from fitting Cox proportional hazards regression model of pastry caterpillar survival without censored data, stratified by location and tree species

\begin{tabular}{lllllll}
\hline & Coef & Exp(coef) & SE(coef) & $\begin{array}{l}\text { Robust } \\
\text { SE }\end{array}$ & Wald & $P$ \\
\hline Colour & 0.108 & 1.114 & 0.1725 & 0.1347 & 0.802 & 0.4228 \\
Eyespot & 0.209 & 1.2324 & 0.1695 & 0.1275 & 1.639 & 0.1012 \\
& & & & & & \\
Colour×eyespot & -0.4559 & 0.6339 & 0.2465 & 0.1812 & -2.516 & 0.0119 \\
\hline
\end{tabular}

In this reanalysis, only prey recovered from the field with peck marks were counted as prey killed, whereas all prey that disappeared over the course of the trial were censored at the time they went missing. Caterpillars pinned to the same tree were clustered in the analysis, and the robust standard error was used to calculate the Wald statistic. 
Table S2-4: Results from Akaike's Information Criterion (AIC) model selection used to determine the factors affecting the number and location of bird pecks on pastry caterpillars deployed in the field

\begin{tabular}{llll}
\hline Model & $d f$ & AIC & $\Delta$ AIC \\
\hline section $\times$ eyespots $+(1 \mid$ individual $)$ & 13 & 2295.665 & 0 \\
section + colour $+(1 \mid$ individual $)$ & 8 & 2331.238 & 35.573 \\
section + eyespots + colour $+(1 \mid$ individual $)$ & 9 & 2332.568 & 36.903 \\
section $+(1 \mid$ individual $)$ & 7 & 2333.523 & 37.858 \\
section + eyespots $+(1 \mid$ individual $)$ & 8 & 2334.536 & 38.871 \\
section + eyespots $\times$ colour $+(1 \mid$ individual $)$ & 10 & 2334.566 & 38.901 \\
section $\times$ colour $+(1 \mid$ individual $)$ & 13 & 2335.619 & 39.954 \\
$(1 \mid$ individual $)$ & 2 & 2782.861 & 487.196 \\
\hline
\end{tabular}

(1|individual): individual as a random factor in the analysis. For marginality reasons, all associated main effects were included in the fitted model when a given interaction was specified. 
Table S2-5: Summary output for the fixed effects from the best fit model explaining the abundance and distribution of pecks on pastry caterpillars with and without eyespots

\begin{tabular}{lrllll}
\hline & Estimate & $\mathrm{SE}$ & \multicolumn{1}{l}{$z$} & $\operatorname{Pr}(>|z|)$ & \\
\hline (Intercept) & 0.11251 & 0.08275 & 1.36 & 0.173949 & \\
section2 & -1.14244 & 0.14244 & -8.02 & $1.06 \mathrm{E}-15$ & $*$ \\
section3 & -1.17274 & 0.14410 & -8.139 & $4.00 \mathrm{E}-16$ & $*$ \\
section4 & -1.37684 & 0.15606 & -8.823 & $2.00 \mathrm{E}-16$ & $*$ \\
section5 & -1.08445 & 0.13937 & -7.781 & $7.19 \mathrm{E}-15$ & $*$ \\
seciton6 & -0.08963 & 0.10137 & -0.884 & 0.376591 & \\
Eyespots & -0.49519 & 0.13388 & -3.699 & 0.000217 & $*$ \\
section2:eyespots & 0.88867 & 0.20169 & 4.406 & $1.05 \mathrm{E}-05$ & $*$ \\
section3:eyespots & 0.29037 & 0.22627 & 1.283 & 0.19939 & \\
section4:eyespots & -0.11863 & 0.27019 & -0.439 & 0.66063 & \\
section5:eyespots & -0.11376 & 0.24053 & -0.473 & 0.636233 & \\
section6:eyespots & 0.78271 & 0.15374 & 5.091 & $3.56 \mathrm{E}-07$ & $*$ \\
\hline
\end{tabular}

$* P<0.05$. 


\section{Appendix C Supplementary material for Chapter 3}

Table S3-1: Insectivorous birds observed in each location during the associated field trail conducted over the summer of 2012 near Kemptville (Sites 1 and 2) and Oxford Mills (Site 3), ON, Canada

\begin{tabular}{|c|c|c|c|c|c|c|}
\hline \multirow[b]{2}{*}{ Bird Species } & \multicolumn{2}{|c|}{ Site 1} & \multicolumn{2}{|c|}{ Site 2} & \multicolumn{2}{|c|}{ Site 3} \\
\hline & June 14 & $\begin{array}{c}\text { July } \\
29 \\
\end{array}$ & $\begin{array}{c}\text { June } \\
20 \\
\end{array}$ & $\begin{array}{c}\text { Aug } \\
20 \\
\end{array}$ & $\begin{array}{c}\text { July } \\
12 \\
\end{array}$ & Aug 28 \\
\hline Bombycilla cedrorum & & & & & & $\mathrm{x}$ \\
\hline Cardinalis cardinalis & & $\mathrm{x}$ & & & & \\
\hline Carduelis tristis & $\mathrm{x}$ & & $\mathrm{x}$ & $\mathrm{x}$ & & \\
\hline Colaptes auratus & & $\mathrm{x}$ & & & & \\
\hline Contopus virens & & & & & $\mathrm{x}$ & \\
\hline Cyanocitta cristata & $\mathrm{x}$ & $\mathrm{x}$ & & & $\mathrm{x}$ & \\
\hline Dendroica Petechia & $\mathrm{x}$ & & & $\mathrm{x}$ & $\mathrm{x}$ & \\
\hline Dumetella carolinensis & $\mathrm{x}$ & & & & & $\mathrm{x}$ \\
\hline Geothlypis trichas & & & $\mathrm{x}$ & & & \\
\hline Melospiza melodia & & $\mathrm{x}$ & $\mathrm{x}$ & & & \\
\hline Mniotilta varia & & $\mathrm{x}$ & & & & \\
\hline $\begin{array}{l}\text { Passerculus } \\
\text { sandwichensis }\end{array}$ & & & $\mathrm{x}$ & & & \\
\hline Pheucticus ludovicianus & & & $\mathrm{x}$ & & & \\
\hline Poecile atricapilla & $\mathrm{x}$ & $\mathrm{x}$ & $\mathrm{x}$ & $\mathrm{x}$ & $\mathrm{x}$ & $\mathrm{x}$ \\
\hline Quiscalus quiscula & $\mathrm{x}$ & & & & & \\
\hline Sayornis phoebe & $\mathrm{x}$ & & & & & \\
\hline Sitta carolinensis & & & $\mathrm{x}$ & & & \\
\hline Spiza americana & & & & $\mathrm{x}$ & & \\
\hline Troglodytes aedon & & & & & & $\mathrm{x}$ \\
\hline Turdus migratorius & & $\mathrm{x}$ & & $\mathrm{x}$ & $\mathrm{x}$ & $\mathrm{x}$ \\
\hline Vireo solitarius & & & & $\mathrm{x}$ & & \\
\hline
\end{tabular}




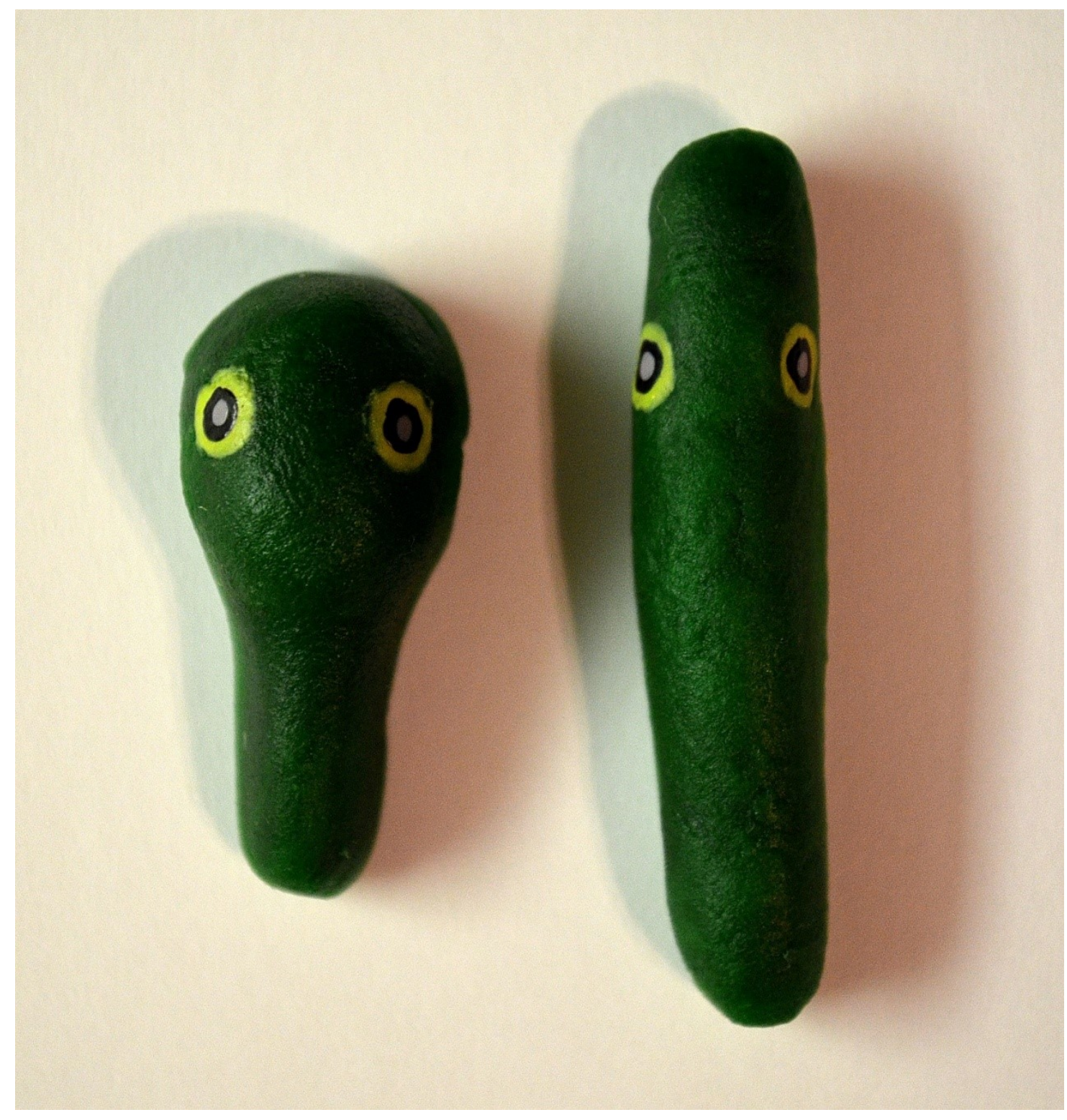

Figure S3-1: Dorsal view of pastry caterpillars in the eyespot treatments used in our experiment. 
Table S3-2: Number of each tree species used to pin pastry caterpillars in each location at each site

\begin{tabular}{llllll}
\hline Site & Location & $\begin{array}{l}\text { Populus } \\
\text { tremuloides }\end{array}$ & $\begin{array}{l}\text { Populus } \\
\text { deltoides }\end{array}$ & $\begin{array}{l}\text { Betula } \\
\text { papyrifera }\end{array}$ & Acer rubrum \\
\hline 1 & 1 & 11 & 0 & 10 & 3 \\
& 2 & 10 & 0 & 10 & 4 \\
2 & 1 & 3 & & & \\
& 2 & 6 & 3 & 5 & 10 \\
& & & & & \\
3 & 1 & 13 & 0 & 0 & 11 \\
& 2 & 20 & 0 & 4 & 0 \\
\hline
\end{tabular}



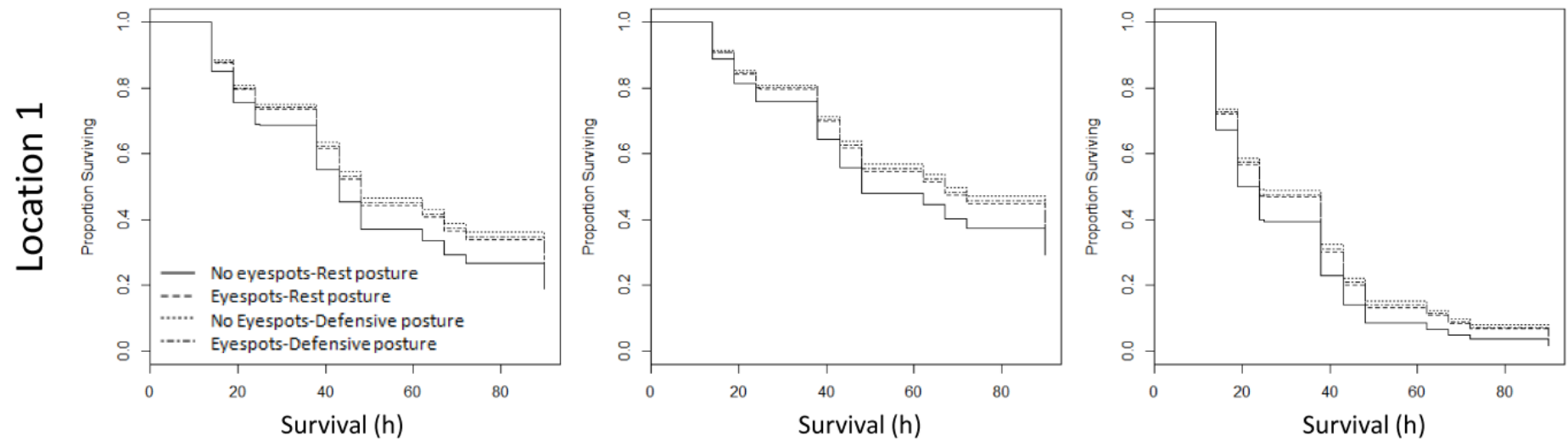

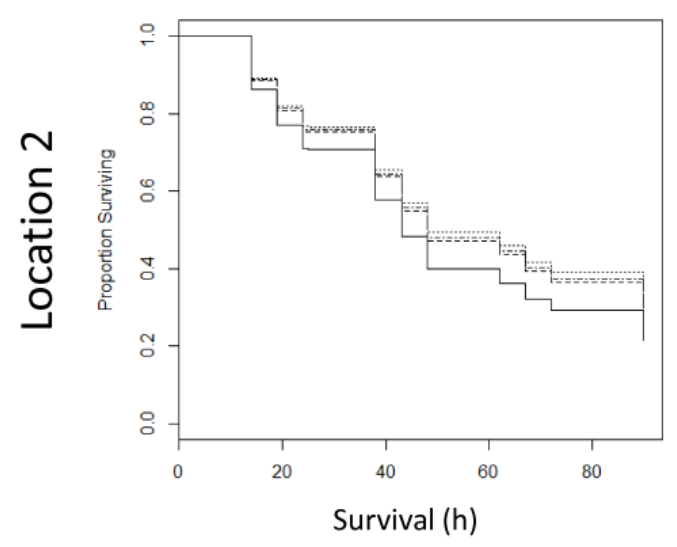

Site 1

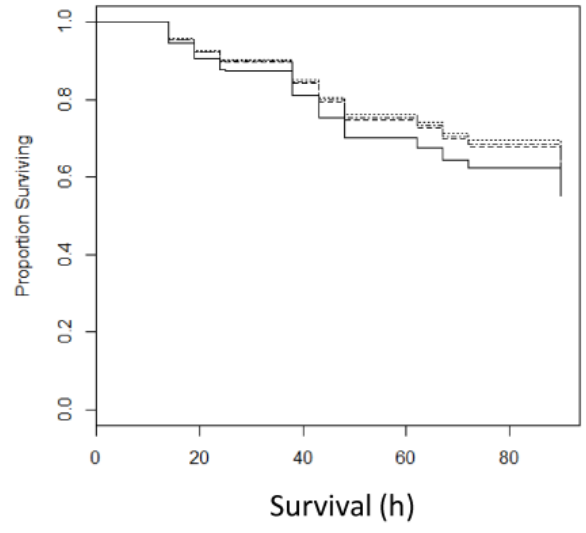

Site 2

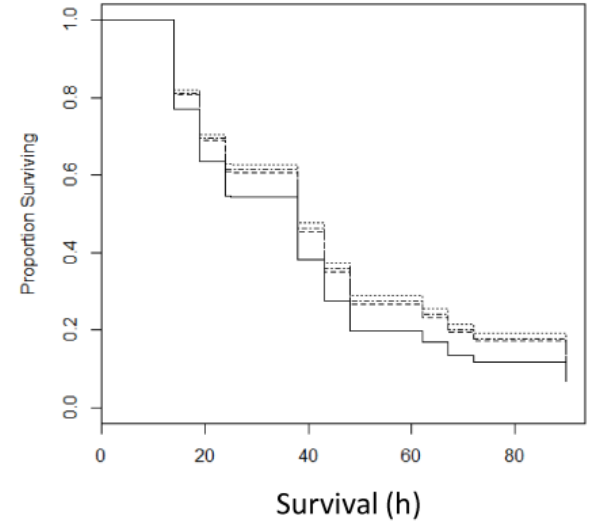

Site 3

Figure S3-2: Fitted cumulative survival charts comparing the survival of four treatments of pastry caterpillars in each of the six locations. Note that each chart is based on $N=96$ baits and, for clarity, these survival curves are fitted without controlling for the tree species on which they were pinned. 

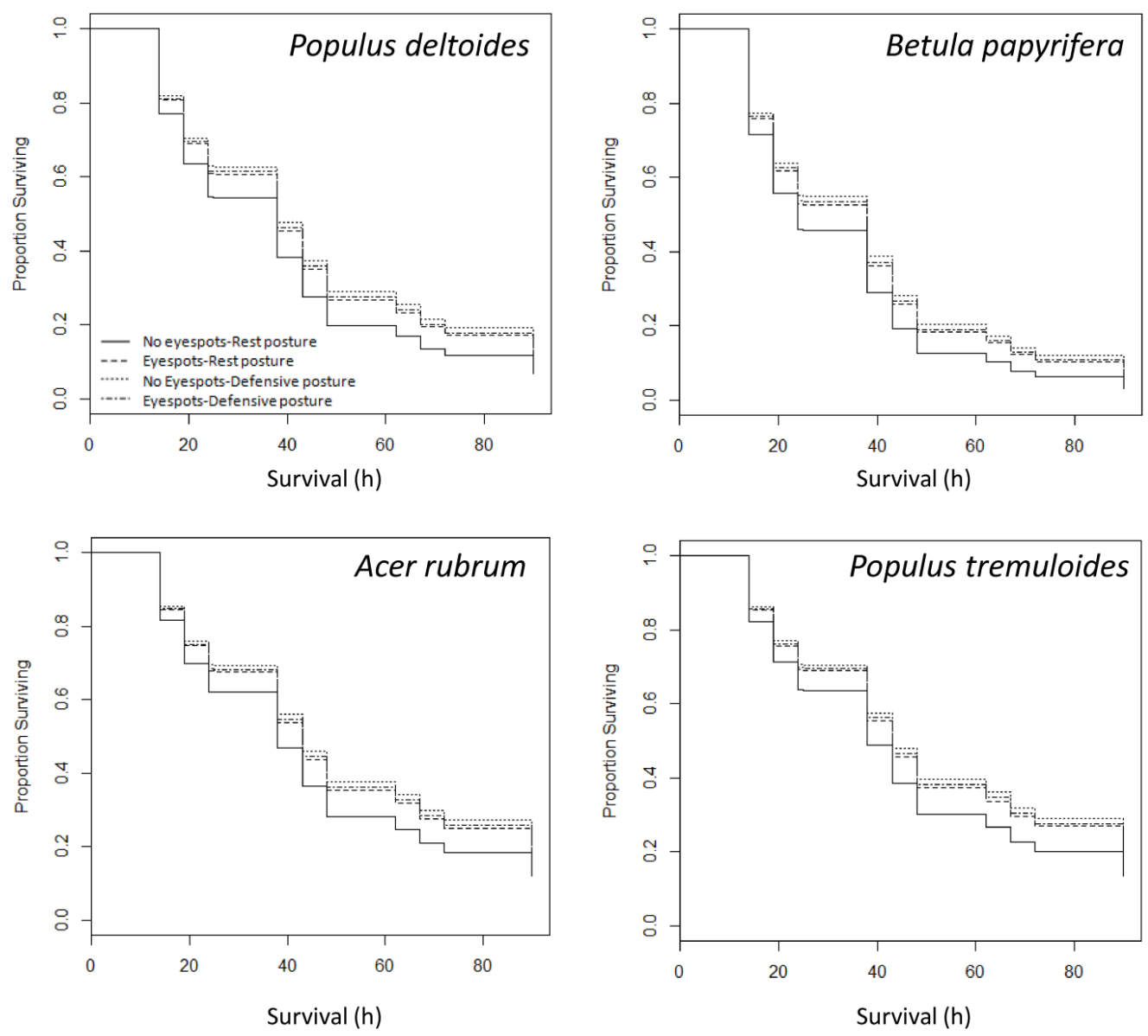

Figure S3-3: Fitted cumulative survival charts comparing the survival of four treatments of pastry caterpillars pinned to different tree species. Note that, for clarity, these survival curves are fitted without controlling for the location where the trials took place. Sample sizes also varied among the four charts: Populus tremuloides: $N=252 ;$ Populus deltoids: $N=80 ;$ Betula papyrifera: $N=120 ;$ Acer rubrum: $N=124$. 
Table S3-3: Results from fitting Cox proportional hazards regression model of pastry caterpillar survival when missing prey were censored, stratified by location and tree species.

\begin{tabular}{lcccccc}
\hline & & & \multicolumn{3}{c}{ Robust } \\
& Coef & $\operatorname{Exp}($ coef) & SE(coef) & SE & Z & $\boldsymbol{P}$ \\
\hline Eyespots & -0.3017 & 0.7395 & 0.205 & 0.1598 & -1.888 & 0.05906 \\
Head Shape & -0.3983 & 0.6715 & 0.2016 & 0.1465 & -2.718 & 0.00656 \\
Eyespots $\times$ Head Shape & 0.2004 & 1.2219 & 0.2986 & 0.1892 & 1.059 & 0.28942 \\
\hline
\end{tabular}

In this reanalysis, only prey recovered from the field with peck marks were counted as prey killed, whereas all prey that disappeared over the course of the trial were censored at the time they went missing. Caterpillars pinned to the same tree were clustered in the analysis. In our analysis we used a binary coding system (i.e., 0/1) for our factors indicating the absence/presence of eyespots or a widened head shape. 
Table S3-4: Results from pair-wise comparisons of the number of pecks in symmetrical body sections. We used a generalized linear model assuming a Poisson distribution with position as a fixed factor and individual as a random effect. Section numbers correspond to the following: Section 1: anterior tip, Section 2: body section with eyespots, Section 3: mid-anterior body, Section 4: mid-posterior body, Section 5: hind-mid posterior body, and Section 6: posterior tip. See Figure 4 in the main text for graphical representation of the data.

\begin{tabular}{lcccl}
\hline & $\begin{array}{c}\text { Section } \\
\text { comparison }\end{array}$ & $\boldsymbol{n}$ & $\boldsymbol{z}$ & $\boldsymbol{P}$-value \\
\hline No eyespots-Rest posture & 1 vs 6 & 56 & -1.434 & 0.152 \\
& 2 vs 5 & 56 & -1.882 & 0.0598 \\
Eyespots-Rest posture & 3 vs 4 & 56 & 0.633 & 0.527 \\
& 1 vs 6 & 43 & 0 & 0.99 \\
No eyespots-Defensive posture & 2 vs 5 & 43 & 0.587 & 0.557 \\
& 3 vs 4 & 43 & -0.707 & 0.48 \\
& 1 vs 6 & 46 & -0.346 & 0.729 \\
Eyespots-Defensive posture & 2 vs 5 & 46 & -1.825 & 0.068 \\
& 3 vs 4 & 46 & -0.284 & 0.776 \\
& 1 vs 6 & 40 & 0.089 & 0.929 \\
& 2 vs 5 & 40 & -3.119 & 0.0018 \\
& 3 vs 4 & 40 & -2.355 & 0.0185 \\
\hline
\end{tabular}




\section{Appendix D Supplementary material for Chapter 4}

Table S4-1: Voucher codes for the caterpillar photographs from the Janzen and Hallwachs (2013) database used in this study.

\begin{tabular}{|c|c|c|c|c|c|c|}
\hline \multirow{2}{*}{ Family } & \multirow[b]{2}{*}{ Genus } & \multirow[b]{2}{*}{ Species } & \multicolumn{2}{|c|}{ Voucher Code - Dorsal View } & \multicolumn{2}{|c|}{ Voucher Code - Lateral View } \\
\hline & & & Rest & Defensive & Rest & Defensive \\
\hline Papilionidae & Papilio & canadensis & Our photos(x10) & Our photos(x10) & Our photos(x10) & Our photos(x10) \\
\hline Saturniidae & Therinia & transversaria & Our photo & Our photo & Our photo & Our photo \\
\hline Sphingidae & Hemeroplanes & triptolemus & & & 97-SRNP-4423-DHJ42550 & $\begin{array}{l}\text { 03-SRNP-11366-DHJ75729, } \\
\text { 97-SRNP-4423-DHJ42596 }\end{array}$ \\
\hline Sphingidae & Xylophanes & acrus & & & 97-SRNP-999-DHJ40683 & 97-SRNP-999-DHJ40677 \\
\hline Sphingidae & Xylophanes & anubus & $\begin{array}{l}\text { 10-SRNP-56098-DHJ478827, } \\
\text { 10-SRNP-56277-DHJ478913 }\end{array}$ & $\begin{array}{l}\text { 02-SRNP-1085-DHJ64470, } \\
\text { 99-SRNP-136-DHJ50036 }\end{array}$ & 10-SRNP-56277-DHJ478914 & 10-SRNP-56277-DHJ478918 \\
\hline Sphingidae & Xylophanes & ceratomioides & & & $\begin{array}{l}\text { 07-SRNP-30429-DHJ419664, } \\
\text { 97-SRNP-1330-DHJ41367 }\end{array}$ & 07-SRNP-30429-DHJ419665 \\
\hline Sphingidae & Xylophanes & chiron & 89-SRNP-45-DHJ10874 & $\begin{array}{l}\text { 84-SRNP-778-DHJ7654, } \\
\text { 84-SRNP-781-DHJ7582 }\end{array}$ & $\begin{array}{l}\text { 03-SRNP-11913-DHJ75997, } \\
\text { 97-SRNP-1115-DHJ40852, } \\
\text { 97-SRNP-1115-DHJ40861 }\end{array}$ & 84-SRNP-778-DHJ7652 \\
\hline Sphingidae & Xylophanes & crotonis & $\begin{array}{l}\text { 88-SRNP-210-DHJ10547, } \\
\text { 97-SRNP-1277-DHJ41214 }\end{array}$ & $\begin{array}{l}\text { 05-SRNP-30799-DHJ403870, } \\
\text { 90-SRNP-131-DHJ12359 }\end{array}$ & $\begin{array}{l}\text { 07-SRNP-36072-DHJ421962, } \\
\text { 97-SRNP-1413-DHJ41460 }\end{array}$ & $\begin{array}{l}\text { 05-SRNP-30799-DHJ403873, } \\
\text { 97-SRNP-991-DHJ40663 }\end{array}$ \\
\hline Sphingidae & Xylophanes & cthulhu & $\begin{array}{l}\text { 04-SRNP-46796-DHJ85724, } \\
\text { 05-SRNP-70564-DHJ409953 }\end{array}$ & $\begin{array}{l}\text { 04-SRNP-46796-DHJ85713, } \\
\text { 04-SRNP-56290-DHJ400264, } \\
\text { 05-SRNP-31108-DHJ403970 }\end{array}$ & 04-SRNP-46796-DHJ401834 & $\begin{array}{l}\text { 05-SRNP-70564-DHJ409942, } \\
\text { 06-SRNP-5738-DHJ411363 }\end{array}$ \\
\hline Sphingidae & Xylophanes & cyrene & $\begin{array}{l}\text { 97-SRNP-1186-DHJ41014, } \\
\text { 97-SRNP-1308-DHJ41297, } \\
\text { 98-SRNP-4167-DHJ45054 }\end{array}$ & $\begin{array}{l}\text { 03-SRNP-20005-DHJ90017, } \\
\text { 04-SRNP-33965-DHJ86617 }\end{array}$ & $\begin{array}{l}\text { 03-SRNP-6092-DHJ74160, } \\
\text { 07-SRNP-30411-DHJ419654, } \\
\text { 07-SRNP-33235-DHJ430174, } \\
\text { 97-SRNP-1308-DHJ41287 }\end{array}$ & 02-SRNP-3532-DHJ67352 \\
\hline Sphingidae & Xylophanes & germen & 97-SRNP-759-DHJ40413 & 98-SRNP-2906-DHJ44485 & $\begin{array}{l}\text { 97-SRNP-759-DHJ40402, } \\
\text { 97-SRNP-759-DHJ40408 }\end{array}$ & 98-SRNP-2906-DHJ44487 \\
\hline Sphingidae & Xylophanes & hannemanni & $\begin{array}{l}\text { 05-SRNP-35718-DHJ406592, } \\
\text { 97-SRNP-1445-DHJ41503, } \\
\text { 98-SRNP-2904-DHJ44482 }\end{array}$ & $\begin{array}{l}\text { 95-SRNP-8588-DHJ25725, } \\
\text { 97-SRNP-1319-DHJ41328 }\end{array}$ & $\begin{array}{l}\text { 97-SRNP-1187-DHJ41027, } \\
\text { 97-SRNP-1189-DHJ41041, } \\
\text { 97-SRNP-1297-DHJ41273, } \\
\text { 97-SRNP-1446-DHJ41510 }\end{array}$ & $\begin{array}{l}\text { 05-SRNP-35718-DHJ406600, } \\
\text { 89-SRNP-782-DHJ11707, } \\
\text { 96-SRNP-7049-DHJ28067 }\end{array}$ \\
\hline Sphingidae & Xylophanes & jocasta & 01-SRNP-2579-DHJ62779 & 03-SRNP-15009-DHJ76686 & & \\
\hline Sphingidae & Xylophanes & juanita & $\begin{array}{l}\text { 81-SRNP-233-DHJ2260, } \\
\text { 84-SRNP-668-DHJ7569, } \\
\text { 84-SRNP-1403-DHJ8202 }\end{array}$ & 84-SRNP-1403-DHJ8203 & & \\
\hline
\end{tabular}




\begin{tabular}{|c|c|c|c|c|c|c|}
\hline Sphingidae & Xylophanes & libya & $\begin{array}{l}\text { 01-SRNP-1685-DHJ57768, } \\
\text { 06-SRNP-5047-DHJ410874, } \\
\text { 08-SRNP-56233-DHJ442748, } \\
\text { 84-SRNP-668-DHJ7569, } \\
\text { 99-SRNP-12438-DHJ51791 }\end{array}$ & 97-SRNP-3065-DHJ42131 & $\begin{array}{l}\text { 01-SRNP-1685-DHJ63211, } \\
\text { 99-SRNP-12438-DHJ51793 }\end{array}$ & 99-SRNP-12438-DHJ51781 \\
\hline Sphingidae & Xylophanes & loelia & $\begin{array}{l}\text { 04-SRNP-41581-DHJ85472, } \\
\text { 08-SRNP-42461-DHJ449689 }\end{array}$ & 08-SRNP-42458-DHJ449660 & $\begin{array}{l}\text { 01-SRNP-4377-DHJ58256, } \\
\text { 04-SRNP-41581-DHJ85463, } \\
\text { 08-SRNP-42461-DHJ449686 }\end{array}$ & 08-SRNP-42458-DHJ449661 \\
\hline Sphingidae & Xylophanes & maculator & $\begin{array}{l}\text { 03-SRNP-6093-DHJ74217, } \\
\text { 05-SRNP-30849-DHJ403890 }\end{array}$ & 84-SRNP-176-DHJ7129 & & \\
\hline Sphingidae & Xylophanes & pluto & $\begin{array}{l}\text { 01-SRNP-10544-DHJ61875, } \\
\text { 81-SRNP-633-DHJ2814, } \\
\text { 92-SRNP-894-DHJ14973, } \\
\text { 92-SRNP-1004-DHJ15019, } \\
\text { 98-SRNP-4966-DHJ45363 }\end{array}$ & 88-SRNP-263-DHJ10610 & $\begin{array}{l}\text { 92-SRNP-1004-DHJ15003, } \\
\text { 98-SRNP-4966-DHJ45359 }\end{array}$ & $\begin{array}{l}\text { 06-SRNP-55100-DHJ409510, } \\
\text { 81-SRNP-633-DHJ2817 }\end{array}$ \\
\hline Sphingidae & Xylophanes & porcus & $\begin{array}{l}\text { 06-SRNP-21539-DHJ411902, } \\
\text { 84-SRNP-367A-DHJ7287, } \\
\text { 95-SRNP-7691-DHJ24958, } \\
\text { 98-SRNP-3198-DHJ44671 }\end{array}$ & 97-SRNP-1185-DHJ41007 & $\begin{array}{l}\text { 06-SRNP-21539-DHJ411904, } \\
\text { 06-SRNP-21542-DHJ411912, } \\
\text { 84-SRNP-367A-DHJ7286 }\end{array}$ & 10-SRNP-56353-DHJ478981 \\
\hline Sphingidae & Xylophanes & tersa & & & $\begin{array}{l}\text { 05-SRNP-34374-DHJ405859, } \\
\text { 97-SRNP-7088-DHJ43706, } \\
\text { 98-SRNP-4176-DHJ45099 }\end{array}$ & $\begin{array}{l}\text { 08-SRNP-40795-DHJ442047, } \\
\text { 97-SRNP-1395-DHJ41417, } \\
\text { 97-SRNP-2397-DHJ41875 }\end{array}$ \\
\hline Sphingidae & Xylophanes & titana & $\begin{array}{l}\text { 02-SRNP-1570-DHJ87871, } \\
\text { 06-SRNP-65788-DHJ425756 }\end{array}$ & $\begin{array}{l}\text { 02-SRNP-1567-DHJ67108, } \\
\text { 02-SRNP-1567-DHJ67129 }\end{array}$ & 02-SRNP-1567-DHJ67106 & 02-SRNP-1567-DHJ67131 \\
\hline Sphingidae & Xylophanes & zurcheri & 98-SRNP-2996-DHJ44551 & 02-SRNP-1942-DHJ67139 & & \\
\hline Papilionidae & Papilio & canadensis & Our photos(x10) & Our photos(x10) & Our photos(x10) & Our photos(x10) \\
\hline
\end{tabular}


Table S4-2: Voucher codes for snake specimens from the collections at the Canadian Museum of Nature photographed for this study.

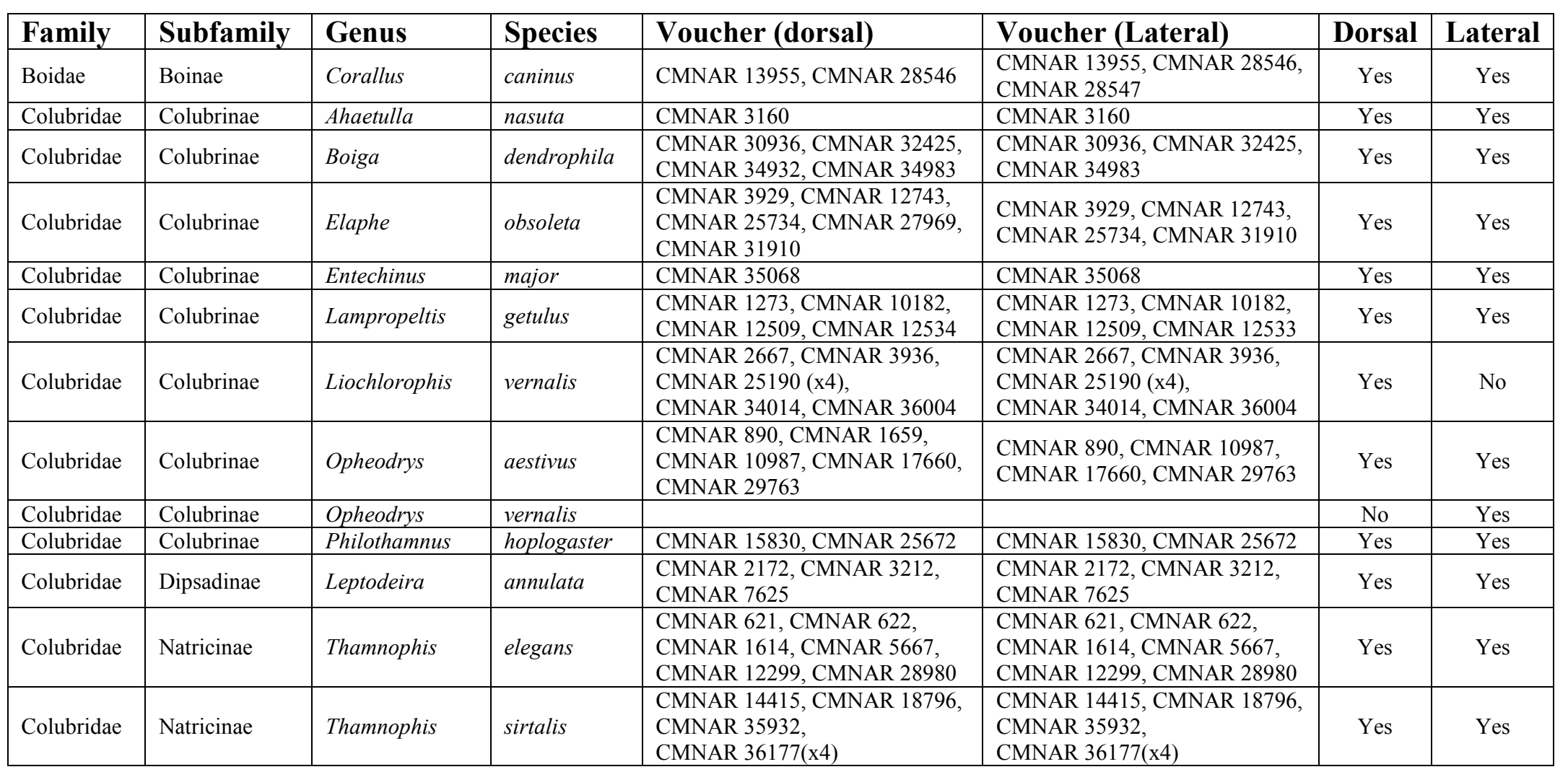




\begin{tabular}{|c|c|c|c|c|c|c|c|}
\hline Colubridae & Xenodontinae & Heterodon & platyrhinos & $\begin{array}{l}\text { CMNAR 14886, CMNAR 15855, } \\
\text { CMNAR 19201, CMNAR 29583, } \\
\text { CMNAR } 34170\end{array}$ & $\begin{array}{l}\text { CMNAR 14886, CMNAR 15855, } \\
\text { CMNAR 19201, CMNAR 29583, } \\
\text { CMNAR } 34170\end{array}$ & Yes & Yes \\
\hline Colubridae & Xenodontinae & Oxybelis & aeneus & CMNAR 27243 & CMNAR 27243 & Yes & Yes \\
\hline Colubridae & Xenodontinae & Oxybelis & fulgidus & CMNAR 34864 & CMNAR 34864 & Yes & Yes \\
\hline Colubridae & Xenodontinae & Sibynomorphus & vagus & CMNAR 35191 & CMNAR 35191 & Yes & Yes \\
\hline Viperidae & Crotalinae & Agkistrodon & acutus & CMNAR 30966, CMNAR 34908 & $\begin{array}{l}\text { CMNAR 30966(x2), CMNAR } \\
34908\end{array}$ & Yes & Yes \\
\hline Viperidae & Crotalinae & Agkistrodon & bilineatus & CMNAR 30950 & & Yes & No \\
\hline Viperidae & Crotalinae & Agkistrodon & contortrix & $\begin{array}{l}\text { CMNAR 12564, CMNAR 34671, } \\
\text { CMNAR 34879, CMNAR } 34962\end{array}$ & $\begin{array}{l}\text { CMNAR 12564, CMNAR 34671, } \\
\text { CMNAR 34879, CMNAR } 34962\end{array}$ & Yes & Yes \\
\hline Viperidae & Crotalinae & Agkistrodon & halys & CMNAR 32423, CMNAR 34960 & CMNAR 32423, CMNAR 34960 & Yes & Yes \\
\hline Viperidae & Crotalinae & Agkistrodon & rhodostoma & CMNAR 34959 & CMNAR 34959 & Yes & Yes \\
\hline Viperidae & Crotalinae & Bothrops & godmani & CMNAR 13798 & CMNAR 13798 & Yes & Yes \\
\hline Viperidae & Crotalinae & Bothrops & lateralis & & CMNAR 30949 & No & Yes \\
\hline Viperidae & Crotalinae & Bothrops & nummifer & CMNAR 34915 & & Yes & No \\
\hline Viperidae & Crotalinae & Bothrops & schlegeli & $\begin{array}{l}\text { CMNAR 32421(x3), CMNAR } \\
34901\end{array}$ & $\begin{array}{l}\text { CMNAR 32421(x3), CMNAR } \\
34901\end{array}$ & Yes & Yes \\
\hline Viperidae & Crotalinae & Crotalus & adamanteus & CMNAR 33995 & CMNAR 33995 & Yes & Yes \\
\hline Viperidae & Crotalinae & Crotalus & atrox & CMNAR 8304 & CMNAR 8304 & Yes & Yes \\
\hline Viperidae & Crotalinae & Crotalus & durissus & CMNAR 31654 & CMNAR 31654 & Yes & Yes \\
\hline Viperidae & Crotalinae & Crotalus & horridus & CMNAR 13954, CMNAR 34319 & CMNAR 13954, CMNAR 34319 & Yes & Yes \\
\hline Viperidae & Crotalinae & Crotalus & scutulatus & CMNAR 12441, CMNAR 34694 & CMNAR 12441, CMNAR 34694 & Yes & Yes \\
\hline Viperidae & Crotalinae & Crotalus & tigris & CMNAR 34412, CMNAR 34981 & CMNAR 34981 & Yes & Yes \\
\hline Viperidae & Crotalinae & Crotalus & viridis & CMNAR 17663 & CMNAR 17663 & Yes & Yes \\
\hline Viperidae & Crotalinae & Lachesis & muta & CMNAR 34902 & CMNAR 34902 & Yes & Yes \\
\hline Viperidae & Crotalinae & Sistrurus & catenatus & CMNAR 13786(x4) & CMNAR 13786(x4) & Yes & Yes \\
\hline Viperidae & Crotalinae & Sistrurus & miliarius & CMNAR 1283 & CMNAR 1283 & Yes & Yes \\
\hline
\end{tabular}


Table S4-3: The standardized coefficients of the linear discriminants.

\begin{tabular}{|c|c|c|c|c|}
\hline $\begin{array}{l}\text { Principal } \\
\text { Component }\end{array}$ & $\begin{array}{c}\text { Caterpillar vs. Snake } \\
\text { (LDA1) }\end{array}$ & $\begin{array}{c}\text { Caterpillar, } \\
\text { Colubridae, or } \\
\text { Viperidae (LDA2) }\end{array}$ & $\begin{array}{c}\text { Caterpillar, } \\
\text { Colubridae, or } \\
\text { Viperidae } \\
\text { (Dorsal PC2 only) }\end{array}$ & Overall model (LDA3) \\
\hline Dorsal PC1 & & & & 21.297 \\
\hline Dorsal PC2 & 20.717 & 25.483 & 17.281 & -14.289 \\
\hline Lateral PC1 & -20.012 & -17.529 & & 13.99 \\
\hline Lateral PC2 & & & & -5.514 \\
\hline
\end{tabular}


Table S4-4: Classification of caterpillars as predicted by the linear discriminant analyses (LDAs). Values in brackets indicate the posterior probability for the assigned classification, expressed as a percentage. LDAs were developed to discriminate between snakes and resting caterpillars (LDA1) or among Colubridae snakes, Viperidae snakes, and resting caterpillars (LDA2) then used to classify the same caterpillars when adopting their defensive posture.

\begin{tabular}{lllllll}
\hline & & & \multicolumn{2}{c}{ LDA1 } & \multicolumn{2}{c}{ LDA2 } \\
\hline Family & Genus & Species & \multicolumn{1}{c}{ Rest } & Defensive & \multicolumn{1}{c}{ Rest } & Defensive \\
\hline Papilionidae & Papilio & canadensis & Snake & Snake & Caterpillar & Viperidae \\
& & & $(55 \%)$ & $(63 \%)$ & $(46 \%)$ & $(73 \%)$ \\
Saturniidae & Therinia & transversaria & $\begin{array}{l}\text { Caterpillar } \\
\text { Caterpillar }\end{array}$ & Caterpillar & Caterpillar \\
& & & $(97 \%)$ & $(84 \%)$ & $(95 \%)$ & $(84 \%)$ \\
Sphingidae & Xylophanes & anubus & Snake & Caterpillar & Colubridae & Caterpillar \\
& & & $(53 \%)$ & $(85 \%)$ & $(50 \%)$ & $(86 \%)$ \\
Sphingidae & Xylophanes & chiron & Caterpillar & Caterpillar & Caterpillar & Caterpillar \\
& & & $(56 \%)$ & $(99 \%)$ & $(57 \%)$ & $(99 \%)$ \\
Sphingidae & Xylophanes & crotonis & Snake & Caterpillar & Colubridae & Caterpillar \\
& & & $(77 \%)$ & $(93 \%)$ & $(62 \%)$ & $(92 \%)$ \\
Sphingidae & Xylophanes & cthulhu & Snake & Snake & Colubridae & Viperidae \\
& & & $(80 \%)$ & $(89 \%)$ & $(50 \%)$ & $(83 \%)$ \\
Sphingidae & Xylophanes & cyrene & Caterpillar & Caterpillar & Caterpillar & Caterpillar \\
& & & $(88 \%)$ & $(51 \%)$ & $(83 \%)$ & $(54 \%)$ \\
Sphingidae & Xylophanes & germen & Caterpillar & Snake & Caterpillar & Viperidae \\
& & & $(85 \%)$ & $(80 \%)$ & $(84 \%)$ & $(81 \%)$ \\
Sphingidae & Xylophanes & hannemanni & Caterpillar & Snake & Caterpillar & Caterpillar \\
& & & $(67 \%)$ & $(54 \%)$ & $(64 \%)$ & $(41 \%)$ \\
Sphingidae & Xylophanes & libya & Snake & Snake & Colubridae & Viperidae \\
& & & $(60 \%)$ & $(99 \%)$ & $(51 \%)$ & $(99 \%)$ \\
Sphingidae & Xylophanes & loelia & Snake & Snake & Colubridae & Viperidae \\
& & & $(58 \%)$ & $(93 \%)$ & $(48 \%)$ & $(93 \%)$ \\
Sphingidae & Xylophanes & pluto & Caterpillar & Snake & Caterpillar & Viperidae \\
& & & $(56 \%)$ & $(92 \%)$ & $(58 \%)$ & $(84 \%)$ \\
Sphingidae & Xylophanes & porcus & Snake & Caterpillar & Colubridae & Caterpillar \\
& & & $(59 \%)$ & $(64 \%)$ & $(56 \%)$ & $(63 \%)$ \\
Sphingidae & Xylophanes & titana & Caterpillar & Caterpillar & Caterpillar & Caterpillar \\
& & & $(56 \%)$ & $(95 \%)$ & $(58 \%)$ & $(95 \%)$ \\
\hline
\end{tabular}




\section{Appendix E Supplementary material for Chapter 6}

Table S6-1: Data sources for body size and eyespot presence/absence in Macroglossinae caterpillars.

\begin{tabular}{|c|c|c|c|c|c|}
\hline Latin Name & Subfamily & Eyespot & $\begin{array}{l}\text { Max } \\
\text { Body Size } \\
\end{array}$ & Source & Voucher \\
\hline Cautethia spuria & Macroglossinae & 0 & 3.4 & Janzen database & 99-SRNP-1121 \\
\hline Unzela japix & Macroglossinae & 0 & 6.4 & Janzen database & 01-SRNP-2399 \\
\hline Aleuron chloroptera & Macroglossinae & 0 & 7.8 & Bura Thesis & \\
\hline Pachygonidia subhamata & Macroglossinae & 0 & 6 & Janzen database & 08-SRNP-32172 \\
\hline Amphion floridensis & Macroglossinae & 0 & 5 & Wagner 2005 & \\
\hline Proserpinus proserpina & Macroglossinae & 1 & 7 & Pittaway \& Kitch & database \\
\hline Sphecodina abbottii & Macroglossinae & 1 & 7.5 & Wagner 2005 & \\
\hline Enyo lugubris & Macroglossinae & 0 & 7 & Janzen database & 05-SRNP-31417 \\
\hline Enyo ocypete & Macroglossinae & 0 & 6.8 & Janzen database & 05-SRNP-56550 \\
\hline Eumorpha phorbas & Macroglossinae & 1 & 8 & Hossie et al 2013 & \\
\hline Eumorpha satellitia & Macroglossinae & 0 & 9 & Janzen database & 09-SRNP-56221 \\
\hline Eumorpha pandorus & Macroglossinae & 1 & 9 & Wagner 2005 & \\
\hline Eumorpha triangulum & Macroglossinae & 0 & 9 & Janzen database & 99-SRNP-283 \\
\hline Kloneus babayaga & Macroglossinae & 0 & 8.2 & Janzen database & 04-SRNP-60316 \\
\hline Pachylia ficus & Macroglossinae & 0 & 11.3 & Janzen database & 08-SRNP-70715 \\
\hline Oryba kadeni & Macroglossinae & 0 & 11 & Janzen database & 07-SRNP-32563 \\
\hline Pachylioides resumens & Macroglossinae & 0 & 8.5 & Janzen database & 78-SRNP-51 \\
\hline Isognathus rimosa & Macroglossinae & 0 & 10 & Janzen database & 78-SRNP-116 \\
\hline Madoryx plutonius & Macroglossinae & 1 & 9.3 & Janzen database & 97-SRNP-32926 \\
\hline Hemeroplanes triptolemus & Macroglossinae & 1 & 8.6 & Janzen database & 03-SRNP-11365 \\
\hline Callionima falcifera & Macroglossinae & 0 & 5 & Janzen database & 11-SRNP-12976 \\
\hline Nyceryx magna & Macroglossinae & 0 & 7 & Janzen database & 09-SRNP-2633 \\
\hline Perigonia stulta & Macroglossinae & 0 & 5.8 & Janzen database & 09-SRNP-36431 \\
\hline Perigonia ilus & Macroglossinae & 0 & 5.8 & Janzen database & 01-SRNP-14555 \\
\hline Eupyrrhoglossum sagra & Macroglossinae & 0 & 5.3 & Janzen database & 06-SRNP-30742 \\
\hline Aellopos tantalus & Macroglossinae & 0 & 5.1 & Tuskes 1980 & \\
\hline Aellopos ceculus & Macroglossinae & 0 & 5.2 & Janzen database & 02-SRNP-7920 \\
\hline Cephonodes hylas & Macroglossinae & 0 & 6.5 & \multicolumn{2}{|c|}{ Pittaway \& Kitching database } \\
\hline Hemaris diffinis & Macroglossinae & 0 & 4.5 & \multicolumn{2}{|l|}{ Wagner 2005} \\
\hline Sphingonaepiopsis gorgoniades & Macroglossinae & 0 & 4 & \multicolumn{2}{|l|}{ Pittaway database } \\
\hline Neogurelca himachala & Macroglossinae & 0 & 5 & \multicolumn{2}{|c|}{ Pittaway \& Kitching database } \\
\hline Angonyx testacea & Macroglossinae & 0 & 6.5 & \multicolumn{2}{|c|}{ Pittaway \& Kitching database } \\
\hline Macroglossum stellatarum & Macroglossinae & 0 & 5 & \multicolumn{2}{|c|}{ Pittaway \& Kitching database } \\
\hline Macroglossum corythus luteata & Macroglossinae & 0 & 7 & \multicolumn{2}{|c|}{ Pittaway \& Kitching database } \\
\hline Daphnis nerii & Macroglossinae & 1 & 13 & \multicolumn{2}{|c|}{ Pittaway \& Kitching database } \\
\hline Deidamia inscriptum & Macroglossinae & 0 & 5 & \multicolumn{2}{|l|}{ Wagner 2005} \\
\hline
\end{tabular}




\begin{tabular}{|c|c|c|c|c|c|}
\hline Acosmeryx naga naga & Macroglossinae & 0 & 10 & \multicolumn{2}{|c|}{ Pittaway \& Kitching database } \\
\hline Enpinanga borneensis & Macroglossinae & 1 & 8 & \multicolumn{2}{|c|}{ Pittaway \& Kitching database } \\
\hline Darapsa myron & Macroglossinae & 0 & 5.5 & \multicolumn{2}{|l|}{ Wagner 2005} \\
\hline Clarina kotschyi kotschyi & Macroglossinae & 0 & 7 & \multicolumn{2}{|l|}{ Pittaway database } \\
\hline Ampelophaga rubiginosa & Macroglossinae & 0 & 10 & \multicolumn{2}{|c|}{ Pittaway \& Kitching database } \\
\hline Gnathothlibus erotus & Macroglossinae & 1 & 8.4 & \multicolumn{2}{|l|}{ Moulds 1981} \\
\hline Hippotion velox & Macroglossinae & 1 & 6 & \multicolumn{2}{|c|}{ Pittaway \& Kitching database } \\
\hline Theretra nessus & Macroglossinae & 1 & 12 & \multicolumn{2}{|c|}{ Pittaway \& Kitching database } \\
\hline Deilephila elpenor elpenor & Macroglossinae & 1 & 8 & \multicolumn{2}{|c|}{ Pittaway \& Kitching database } \\
\hline Pergesa acteus & Macroglossinae & 1 & 7 & \multicolumn{2}{|c|}{ Pittaway \& Kitching database } \\
\hline Theretra oldenlandiae & Macroglossinae & 1 & 8 & \multicolumn{2}{|c|}{ Pittaway \& Kitching database } \\
\hline Theretra silhetensis & Macroglossinae & 1 & 8 & \multicolumn{2}{|c|}{ Pittaway \& Kitching database } \\
\hline Rhagastis mongoliana & Macroglossinae & 1 & 8.4 & \multicolumn{2}{|c|}{ Pittaway \& Kitching database } \\
\hline Theretra latreillii lucasii & Macroglossinae & 1 & 6 & \multicolumn{2}{|c|}{ Pittaway \& Kitching database } \\
\hline Theretra alecto & Macroglossinae & 1 & 11 & \multicolumn{2}{|l|}{ Pittaway database } \\
\hline Cechetra lineosa & Macroglossinae & 1 & 10 & \multicolumn{2}{|c|}{ Pittaway \& Kitching database } \\
\hline Theretra clotho clotho & Macroglossinae & 1 & 8.5 & \multicolumn{2}{|c|}{ Pittaway \& Kitching database } \\
\hline Hippotion celerio & Macroglossinae & 1 & 9 & \multicolumn{2}{|l|}{ Pittaway database } \\
\hline Hyles hippophaes hippophaes & Macroglossinae & 0 & 8 & \multicolumn{2}{|l|}{ Pittaway database } \\
\hline Hyles lineata & Macroglossinae & 0 & 7 & \multicolumn{2}{|l|}{ Wagner 2005} \\
\hline Xylophanes tyndarus & Macroglossinae & 1 & 8 & Janzen database & 09-SRNP-56411 \\
\hline Xylophanes chiron & Macroglossinae & 1 & 7.7 & Janzen database & 04-SRNP-1496 \\
\hline Xylophanes tersa & Macroglossinae & 1 & 7 & Janzen database & 05-SRNP-34374 \\
\hline Xylophanes porcus & Macroglossinae & 1 & 9 & Janzen database & 06-SRNP-21542 \\
\hline Xylophanes falco & Macroglossinae & 1 & 7.3 & \multicolumn{2}{|c|}{ Kawahara, unpublished } \\
\hline Xylophanes loelia & Macroglossinae & 1 & 8.1 & Janzen database & 04-SRNP-41581 \\
\hline Xylophanes maculator & Macroglossinae & 1 & 8 & Janzen database & 05-SRNP-30849 \\
\hline Xylophanes crotonis & Macroglossinae & 1 & 9 & Janzen database & 97-SRNP-1321 \\
\hline Xylophanes anubus & Macroglossinae & 1 & 8.5 & Janzen database & 01-SRNP-6893 \\
\hline
\end{tabular}


Table 6-2: Insectivorous birds observed in each location during the associated field trail conducted over the summer of 2013 near Kemptville (Sites 1 and 2) and Oxford Mills (Site 3), ON, Canada

\begin{tabular}{|c|c|c|c|c|c|c|}
\hline \multirow[b]{2}{*}{ Bird Species } & \multicolumn{2}{|c|}{ Site 1} & \multicolumn{2}{|c|}{ Site 2} & \multicolumn{2}{|c|}{ Site 3} \\
\hline & May 8 & June 19 & May 25 & July 21 & June 3 & Sept 4 \\
\hline Bombycilla cedrorum & & & & & & $\mathrm{x}$ \\
\hline Cardinalis cardinalis & & & $\mathrm{x}$ & & & \\
\hline Carduelis tristis & & & $\mathrm{x}$ & & $\mathrm{x}$ & \\
\hline Contopus virens & & & & & & $\mathrm{x}$ \\
\hline Cyanocitta cristata & & & $\mathrm{x}$ & $\mathrm{x}$ & $\mathrm{x}$ & $\mathrm{x}$ \\
\hline Dendroica Petechia & & & & & $\mathrm{x}$ & \\
\hline Dumetella carolinensis & & & $\mathrm{x}$ & & $\mathrm{x}$ & \\
\hline Geothlypis trichas & & $\mathrm{x}$ & $\mathrm{x}$ & & $\mathrm{x}$ & \\
\hline Melospiza melodia & & $\mathrm{x}$ & & & & $\mathrm{x}$ \\
\hline Passerculus sandwichensis & & & & & $\mathrm{x}$ & \\
\hline Pheucticus ludovicianus & & $\mathrm{x}$ & $\mathrm{x}$ & $\mathrm{x}$ & & \\
\hline Poecile atricapilla & $\mathrm{x}$ & $\mathrm{x}$ & $\mathrm{x}$ & $\mathrm{x}$ & $\mathrm{x}$ & $\mathrm{x}$ \\
\hline Quiscalus quiscula & & & & & $\mathrm{x}$ & \\
\hline Sitta carolinensis & & & & $\mathrm{x}$ & & $\mathrm{x}$ \\
\hline Turdus migratorius & $\mathrm{x}$ & $\mathrm{x}$ & $\mathrm{x}$ & $\mathrm{x}$ & $\mathrm{x}$ & $\mathrm{x}$ \\
\hline Vireo solitarius & & & & & & \\
\hline Zonotrichia albicollis & $\mathrm{x}$ & $\mathrm{x}$ & & & & \\
\hline Passerina cyanea & & & & & $\mathrm{x}$ & \\
\hline Setophaga pensylvanica & & & & & $\mathrm{x}$ & $\mathrm{x}$ \\
\hline Agelaius phoeniceus & & & & & $\mathrm{x}$ & \\
\hline Catharus fuscescens & & $\mathrm{x}$ & & & & \\
\hline Setophaga virens & & & & $\mathrm{x}$ & & \\
\hline Myiarchus crinitus & & & & $\mathrm{x}$ & & \\
\hline Troglodytes hiemalis & & & & $\mathrm{x}$ & & \\
\hline Eremophila alpestris & & & & & & $\mathrm{x}$ \\
\hline Vireo olivaceus & & & & & & $\mathrm{x}$ \\
\hline
\end{tabular}




\section{References}

Abbott, K. R. \& Sherratt, T. N. 2013. Optimal sampling and signal detection: unifying models of attention and speed-accuracy trade-offs. Behavioral Ecology, 24, 605-616.

Allen, T. J., Brock, J. P. \& Glassberg, J. 2005. Caterpillars in the field and garden: a field guide to the butterfly caterpillars of North America. New York, NY: Oxford University Press, Inc.

Aronsson, M. \& Gamberale-Stille, G. 2012. Colour and pattern similarity in mimicry: evidence for a hierarchical discriminative learning of different components. Animal Behaviour, 84, 881-887.

Bates, H. 1862. Contributions to an insect fauna of the Amazon Valley: Lepidoptera: Heliconiae. Transactions of the Linnean Society of London, 23, 495-564.

Bates, D., Maechler, M. \& Bolker, B. M. 2011. Linear mixed-effects models using S4 classes.

Benitez-Vieyra, S., Medina, a. M. \& Cocucci, a. a. 2009. Variable selection patterns on the labellum shape of Geoblasta pennicillata, a sexually deceptive orchid. Journal of Evolutionary Biology, 22, 2354-2362.

Blest, A. D. 1957. The function of eyespot patterns in the Lepidoptera. Behaviour, 11, 209-256.

Blut, C., Wilbrandt, J., Fels, D., Girgel, E. I. \& Lunau, K. 2012. The "sparkle" in fake eyes - the protective effect of mimic eyespots in lepidoptera. Entomologia Experimentalis et Applicata, 143, 231-244.

Breuker, C. J. \& Brakefield, P. M. 2002. Female choice depends on size but not symmetry of dorsal eyespots in the butterfly Bicyclus anynana. Proceedings. Biological sciences / The Royal Society, 269, 1233-9.

Brower, J. 1958. Experimental studies of mimicry in some North American butterflies: Part II. Battus philenor and Papilio troilus, P. polyxenes and P. glaucus. Evolution, 12, $123-136$.

Buckler, W. 1887. The larvae of the British butterflies and moths - volume II The sphinges or hawk-moths and part of the bombyces. 2nd edn. London: Printed for the Bay Society.

Bura, V. L. 2010. Sound production in Bombycoidea caterpillars: a comparative study of taxonomic diversity, mechanisms and function. Carleton University. 
Caro, T. 2005. Antipredator defenses in birds and mammals. Chicago: Chicago University Press.

Charlesworth, D. \& Charlesworth, B. 1975. Theoretical Genetics of Batesian Mimicry I. Single-locus models. Journal of theoretical biology, 55, 283-303.

Chittka, L. \& Osorio, D. 2007. Cognitive dimensions of predator responses to imperfect mimicry. PLoS biology, 5, e339.

Church, S. C., Jowers, M. \& Allen, J. A. 1997. Does prey dispersion affect frequencydependent predation by wild birds? Oecologia, 111, 292-296.

Condamine, F. L., Sperling, F. a H., Wahlberg, N., Rasplus, J.-Y. \& Kergoat, G. J. 2012. What causes latitudinal gradients in species diversity? Evolutionary processes and ecological constraints on swallowtail biodiversity. Ecology letters, 15, 267-77.

Cott, H. B. 1940. Adaptive coloration in animals. London: Methuen \& Co. Ltd.

Cox, D. R. 1972. Regression models and life-tables. Journal of the Royal Statistical Society. Series B (Methodological), 34, 187-220.

Cozzolino, S. \& Widmer, A. 2005. Orchid diversity: an evolutionary consequence of deception? Trends in ecology \& evolution, 20, 487-494.

Curio, E. 1965. Die Schlangenmimikry einer südamerikanischen Schwärmerraupe. Natur und Museum, 95, 207-211.

Darst, C. R., Menéndez-Guerrero, P. a, Coloma, L. a \& Cannatella, D. C. 2005. Evolution of dietary specialization and chemical defense in poison frogs (Dendrobatidae): a comparative analysis. The American naturalist, 165, 56-69.

Darwin, C. 1882. The decent of man, and selection in relation to sex. 2nd edn. London: John Murray.

Deloache, J. S. \& Lobue, V. 2009. The narrow fellow in the grass: human infants associate snakes and fear. Developmental science, 12, 201-207.

Dittrich, W., Gilbert, F. S., Green, P., McGregor, P. \& Grewcock, D. 1993. Imperfect Mimicry: A Pigeon's Perspective. Proceedings of the Royal Society B: Biological Sciences, 251, 195-200.

Duncan, A. C. J. \& Sheppard, P. M. 1965. Sensory discrimination and its role in the evolution of batesian mimicry. Behaviour, 24, 269-282.

Dunford, J. C. \& Barbara, K. A. 2008. Tetrio Sphinx, Giant Gray Sphinx, Frangipani Hornworm, Pseudosphinx tetrio (Linnaeus) (Insecta: Lepidoptera: Sphingidae). 
Edmunds, M. 1974. Defence in animals: a survey of anti-predator defences. Harlow, Essex \& NY: Longman Group Ltd.

Edmunds, M. 2000. Why are there good and poor mimics? Biological Journal of the Linnean Society, 70, 459-466.

Edmunds, M. \& Dewhirst, R. A. 1994. The survival value of countershading with wild birds as predators. Biological Journal of the Linnean Society, 51, 447-452.

Endler, J. A. 1981. An overview of the relationships between mimicry and crypsis. Biological Journal of the Linnean Society, 16, 25-31.

Frankfater, C., Tellez, M. R. \& Slattery, M. 2009. The scent of alarm: ontogenetic and genetic variation in the osmeterial gland chemistry of Papilio glaucus (Papilionidae) caterpillars. Chemoecology, 19, 81-96.

Gamberale-Stille, G., Balogh, A. C. V, Tullberg, B. S. \& Leimar, O. 2012. Feature saltation and the evolution of mimicry. Evolution, 66, 807-17.

Gilbert, L. E. 1991. Biodiversity of a Central American Heliconius community: pattern, process, and problems. In: Plant-animal interactions: evolutionary ecology in tropical and temperate regions, (Ed. by P. W. Price, T. M. Lewinsohn, G. W. Fernandes, \& W. W. Benson), pp. 403-427. New York, NY: Wiley.

Gluckman, T.-L. \& Mundy, N. I. 2013. Cuckoos in raptors' clothing: barred plumage illuminates a fundamental principle of Batesian mimicry. Animal Behaviour,

Gould, S. J. \& Vrba, E. S. 1982. Exaptation-a missing term in the science of form. Paleobiology, 8, 4-15.

Hagman, M. \& Forsman, A. 2003. Correlated evolution of conspicuous coloration and body size in poison frogs (Dendrobatidae). Evolution; international journal of organic evolution, 57, 2904-10.

Hall, P., Layberry, R. \& Lafontaine, D. 1996. Checklist of the butterflies of the Ottawa district. Ottawa.

Harper, G. R. \& Pfennig, D. W. 2007. Mimicry on the edge: why do mimics vary in resemblance to their model in different parts of their geographical range? Proceedings. Biological sciences / The Royal Society, 274, 1955-61.

Hawkins, L. A., Magurran, A. E. \& Armstrong, J. D. 2004. Innate predator recognition in newly-hatched Atlantic salmon. Behaviour, 141, 1249-1262.

Hayward, K. J. 1929. Larval descriptions from the Argentine. The Entomologist's Record and Journal of Variation, 40/41, 143-144. 
Heinrich, B. 1979. Foraging strategies of caterpillars: leaf damage and possible predator avoidance strategies. Oecologia, 42, 325-337.

Higginson, A. D., De Wert, L., Rowland, H. M., Speed, M. \& Ruxton, G. D. 2012. Masquerade is associated with polyphagy and larval overwintering in Lepidoptera. Biological Journal of the Linnean Society, 106, 90-103.

Höhna, S. 2014. Likelihood inference of non-constant diversification rates with incomplete taxon tampling. PloS ONE, 9, 17-20.

Hossie, T. J. \& Sherratt, T. N. 2012. Eyespots interact with body colour to protect caterpillar-like prey from avian predators. Animal Behaviour, 84, 167-173.

Hossie, T. J. \& Sherratt, T. N. 2013. Defensive posture and eyespots deter avian predators from attacking caterpillar models. Animal Behaviour, 86, 383-389.

Hossie, T. J. \& Sherratt, T. N. 2014. Does defensive posture increase mimetic fidelity of caterpillars with eyespots to their putative snake models? Current Zoology, 60, 76-89.

Hultgren, K. M. \& Stachowicz, J. J. 2009. Evolution of decoration in majoid crabs: a comparative phylogenetic analysis of the role of body size and alternative defensive strategies. The American naturalist, 173, 566-78.

Janzen, D. H. 1980. Two Potential Coral Snake Mimics in a Tropical Deciduous Forest. Biotropica, 12, 77-78.

Janzen, D. H. 1988. Ecological characterization of a Costa Rican dry forest caterpillar fauna. Biotropica, 20, 120-135.

Janzen, D. H. \& Hallwachs, W. 2011. Joining inventory by parataxonomists with DNA barcoding of a large complex tropical conserved wildland in northwestern Costa Rica. PloS one, 6, e18123.

Janzen, D. H. \& Hallwachs, W. 2014. Dynamic database for an inventory of the macrocaterpillar fauna, and its food plants and parasitoids, of Area de Conservacion Guanacaste (ACG), northwestern Costa Rica (nn-SRNP-nnnnn voucher codes).

Janzen, D. H., Hajibabaei, M., Burns, J. M., Hallwachs, W., Remigio, E. \& Hebert, P. D. N. 2005. Wedding biodiversity inventory of a large and complex Lepidoptera fauna with DNA barcoding. Philosophical transactions of the Royal Society of London. Series B, Biological sciences, 360, 1835-45.

Janzen, D. H., Hallwachs, W. \& Burns, J. M. 2010. A tropical horde of counterfeit predator eyes. Proceedings of the National Academy of Sciences of the United States of America, 107, 11659-11665. 
Johnson, J. A. \& Brodie, E. D. 1975. The selective advantage of the defensive posture of the newt, Taricha granulosa. American Midland Naturalist, 93, 139-148.

Kang, C.-K., Moon, J.-Y., Lee, S.-I. \& Jablonski, P. G. 2012. Camouflage through an active choice of a resting spot and body orientation in moths. Journal of evolutionary biology, 25, 1695-1702.

Kapan, D. D. 2001. Three-butterfly system provides a field test of Müllerian mimicry. Nature, 409, 338-340.

Karplus, I. \& Algom, D. 1981. Visual cues for predator face recognition by reef fishes. Zeitschrift für Tierpsychologie, 55, 343-364.

Kikuchi, D. W. \& Pfennig, D. W. 2009. High-model abundance may permit the gradual evolution of Batesian mimicry: an experimental test. Proceedings of the Royal Society B: Biological Sciences, 277, 1041-8.

Kjernsmo, K. \& Merilaita, S. 2013. Eyespots divert attacks by fish Eyespots divert attacks by fish.

Kodandaramaiah, U. 2011. The evolutionary significance of butterfly eyespots. Behavioral Ecology, 22, 1264-1271.

Kodandaramaiah, U., Vallin, A. \& Wiklund, C. 2009. Fixed eyespot display in a butterfly thwarts attacking birds. Animal Behaviour, 77, 1415-1419.

Kodandaramaiah, U., Lindenfors, P. \& Tullberg, B. S. 2013. Deflective and intimidating eyespots: a comparative study of eyespot size and position in Junonia butterflies. Ecology and Evolution, n/a-n/a.

Lederhouse, R. C. 1990. Avoiding the hunt: Primary defenses of lepidopteran caterpillars. In: Insect Defenses: Adaptive Mechanisms and Strategies of Prey and Predators, (Ed. by D. L. Evans \& J. O. Schmidt), pp. 175-189. Albany: State University of New York Press.

Lenzi-Mattos, R., Antoniazzi, M. M., Haddad, C. F. B., Tambourgi, D. V, Rodrigues, M. T. \& Jared, C. 2005. The inguinal macroglands of the frog Physalaemus nattereri (Leptodactylidae): structure, toxic secretion and relationship with deimatic behaviour. Journal of Zoology, 266, 385-394.

Leslie, A. \& Berenbaum, M. 1990. Role of the osmeterial gland in swallowtail larvae (Papilionidae) in defense against an avian predator. Journal of the Lepidopterists' Society, 44, 245-251. 
Lovegrove, B. G. 2001. The evolution of body armor in mammals: plantigrade constraints of large body size. Evolution; international journal of organic evolution, 55, 1464-73.

Lyytinen, A., Brakefield, P. M. \& Mappes, J. 2003. Significance of butterfly eyespots as an anti-predator device in ground-based and aerial attacks. Oikos, 373-379.

Lyytinen, A., Brakefield, P. M., Lindström, L. \& Mappes, J. 2004. Does predation maintain eyespot plasticity in Bicyclus anynana? Proceedings of the Royal Society B: Biological Sciences, 271, 279-283.

Mallet, J. \& Joron, M. 1999. Evolution of diversity in warning color and mimicry: polymorphisms, shifting balance, and Speciation. Annual Review of Ecology and Systematics, 30, 201-233.

Mallet, J., Barton, N. H. \& Mar, N. 1989. Strong Natural Selection in a Warning-Color Hybrid Zone. Evoution, 43, 421-431.

Marsh, N. \& Rothschild, M. 2009. Aposematic and cryptic Lepidoptera tested on the mouse. Journal of Zoology, 174, 89-122.

Martins, E. \& Hansen, T. 1997. Phylogenies and the comparative method: a general approach to incorporating phylogenetic information into the analysis of interspecific data. American Naturalist, 149, 646-667.

Mayer, R. 1985. The artist's handbook of materials and techniques. 4th edn. New York, NY: Viking Penguin Inc.

Mead, A. 1976. Vascularity in the reptilian spectacle. Investigative Ophthalmology \& Visual Science, 15, 587-591.

Merilaita, S., Vallin, A., Kodandaramaiah, U., Dimitrova, M., Ruuskanen, S. \& Laaksonen, T. 2011. Number of eyespots and their intimidating effect on naive predators in the peacock butterfly. Behavioral Ecology, 22, 1326-1331.

Merle, H., Suchocki, D., Gérard, M. \& Donnio, A. 2001. Kératite par projection de polis de chenille de Pseuosphinx tetrio. Journal Français d'Ophtalmologie, 24, 635-638.

Moss, A. M. 1912. On the Sphingidae of Peru. Transactions of the Zoological Society of London, 20, 73-135.

Moss, A. M. 1920. The Sphingidae of Para, Brazil. Novitates Zoologicae, 27, 333-424.

Müller, F. 1879. Ituna and Thyridia; a remarkable case of mimicry in butterflies. (R. Meldola translation). Proclamations of the Entomological Society of London, 1879, 2029. 
Nelson, X. J. \& Jackson, R. R. 2006. Vision-based innate aversion to ants and ant mimics. Behavioral Ecology, 17, 676-681.

Nelson, X. J. \& Jackson, R. R. 2009. Collective Batesian mimicry of ant groups by aggregating spiders. Animal Behaviour, 78, 123-129.

Nelson, X. J., Jackson, R. R., Li, D., Barrion, A. T. \& Edwards, G. B. 2006. Innate aversion to ants (Hymenoptera: Formicidae) and ant mimics: experimental findings from mantises (Mantodea). Biological Journal of the Linnean Society, 88, 23-32.

Nicholson, A. J. 1927. A new theory of mimicry in insects. Australian Zoology, 4, 10104.

Nyffeler, M. \& Knörnschild, M. 2013. Bat predation by spiders. PloS one, 8, e58120.

O'Hanlon, J. C., Holwell, G. I. \& Herberstein, M. E. 2014. Pollinator deception in the orchid mantis. The American naturalist, 183, 126-32.

Oliver, J. C., Robertson, K. a \& Monteiro, A. 2009. Accommodating natural and sexual selection in butterfly wing pattern evolution. Proceedings. Biological sciences / The Royal Society, 276, 2369-75.

Olofsson, M., Vallin, A., Jakobsson, S. \& Wiklund, C. 2010. Marginal eyespots on butterfly wings deflect bird attacks under low light intensities with UV wavelengths. PloS one, $\mathbf{5}$, e10798.

Olofsson, M., Løvlie, H., Tibblin, J., Jakobsson, S. \& Wiklund, C. 2012. Eyespot display in the peacock butterfly triggers antipredator behaviors in naïve adult fowl. Behavioral Ecology, 24, 305-310.

Papadopulos, A. S. T., Powell, M. P., Pupulin, F., Warner, J., Hawkins, J. A., Salamin, N., Chittka, L., Williams, N. H., Whitten, W. M., Loader, D., Valente, L. M., Chase, M. W., Savolainen, V. \& B, P. R. S. 2013. Convergent evolution of floral signals underlies the success of Neotropical orchids Convergent evolution of floral signals underlies the success of Neotropical orchids. Proceedings of the Royal Society B: Biological Sciences, 280, 20130960.

Paradis, E. \& Claude, J. 2002. Analysis of comparative data using generalized estimating equations. Journal of Theoretical Biology, 218, 175-185.

Penney, H. D., Hassall, C., Skevington, J. H., Abbott, K. R. \& Sherratt, T. N. 2012. A comparative analysis of the evolution of imperfect mimicry. Nature, 483, 461-4.

Penney, H. D., Hassall, C., Skevington, J. H., Lamborn, B. \& Sherratt, T. N. 2014. The relationship between morphological and behavioral mimicry in hover flies (Diptera: Syrphidae). The American Naturalist, 183, 281-289. 
Pfennig, D. W. \& Mullen, S. P. 2010. Mimics without models: causes and consequences of allopatry in Batesian mimicry complexes. Proceedings. Biological sciences / The Royal Society, 277, 2577-85.

Pickens, A. 1927. A Possible Serpent-Like Mimic. Copeia, 162, 24-25.

Pough, F. H. 1988. Mimicry of vertebrates: are the rules different? The American Naturalist, 131 Supple, S67-S102.

Poulton, E. B. 1890. The colours of animals: their meaning and use especially considered in the case of insects. London: Kegan Paul, Trench, Trubner, \& Co. Ltd.

Prudic, K. L., Oliver, J. C. \& Sperling, F. a H. 2007. The signal environment is more important than diet or chemical specialization in the evolution of warning coloration. Proceedings of the National Academy of Sciences of the United States of America, 104, 19381-6.

R Development Core Team. 2014. R: a language and environment for statistical computing computer program.

Remmel, T. \& Tammaru, T. 2009. Size-dependent predation risk in tree-feeding insects with different colouration strategies: a field experiment. The Journal of animal ecology, 78, 973-80.

Revell, L. J. 2010. Phylogenetic signal and linear regression on species data. Methods in Ecology and Evolution, 1, 319-329.

Revell, L. J. 2012. phytools: An R package for phylogenetic comparative biology (and other things). Methods in Ecology and Evolution, 3, 217-223.

Ritland, D. \& Brower, L. P. 1991. The viceroy butterfly is not a Batesian mimic. Nature, 350, 497-498.

Rohlf, F. J. 2006. tpsDig2.

Rothschild, M. 1984. Aide memoire mimicry. Ecological entomology, 9, 311-319.

Rowland, H. M., Speed, M., Ruxton, G. D., Edmunds, M., Stevens, M. \& Harvey, I. F. 2007. Countershading enhances cryptic protection: an experiment with wild birds and artificial prey. Animal Behaviour, 74, 1249-1258.

Rowland, H. M., Cuthill, I. C., Harvey, I. F., Speed, M. \& Ruxton, G. D. 2008. Can't tell the caterpillars from the trees: countershading enhances survival in a woodland. Proceedings of the Royal Society B: Biological Sciences, 275, 2539-45. 
Rudh, A. 2013. Loss of conspicuous coloration has co-evolved with decreased body size in populations of poison dart frogs. Evolutionary Ecology, 27, 755-767.

Ruxton, G. D. 2005. Intimidating butterflies. Trends in ecology \& evolution, 20, 276278.

Ruxton, G. D., Sherratt, T. N. \& Speed, M. 2004a. Avoiding attack: the evolutionary ecology of crypsis, warning signals \& mimicry. New York, NY: Oxford University Press, Inc.

Ruxton, G. D., Speed, M. \& Kelly, D. J. 2004b. What, if anything, is the adaptive function of countershading? Animal Behaviour, 68, 445-451.

San Martin, G., Bacquet, P. \& Nieberding, C. M. 2011. Mate choice and sexual selection in a model butterfly species, Bicyclus anynana: state of the art. Proceedings of the Netherlands Entomological Society Meeting, 22, 9-22.

Santos, J. C., Coloma, L. a \& Cannatella, D. C. 2003. Multiple, recurring origins of aposematism and diet specialization in poison frogs. Proceedings of the National Academy of Sciences of the United States of America, 100, 12792-7.

Saul-gershenz, L. S. \& Millar, J. G. 2006. Phoretic nest parasites use sexual deception to obtain transport to their host's nest. Proceedings of the National Academy of Sciences of the United States of America, 103, 14039-14044.

Scott, J. A. 1986. The butterflies of North America. Stanford, CA: Stanford University Press.

Scriber, J. M., Lederhouse, R. C. \& Dowell, R. V. 1995. Hybridization studies with North American swallowtails. In: Swallowtail butterflies: their ecology and evolution, (Ed. by J. M. Scriber, Y. Tsubaki, \& R. C. Lederhouse), pp. 269-281. Gainesville, FL: Scientific Publishers.

Sheets, H. D. 2011a. CoordGen7a.

Sheets, H. D. 2011b. PCAGen7a.

Sheppard, P. M. 1962. Some aspects of the geography, genetics, and taxonomy of a butterfly. In: Taxonomy and Geography, (Ed. by D. Nichols), pp. 135-152. Syst. Assoc. Publ. No. 4.

Sheppard, P. M. 1967. Natural Selection and Heredity. 3rd ed. edn. London: Hutchinson.

Sherratt, T. N. 2002. The evolution of imperfect mimicry. Behavioral Ecology, 13, 821826. 
Sherratt, T. N. 2008. The evolution of müllerian mimicry. Naturwissenschaften, 95, 681-695.

Shirota, Y. 1980. Protective function of eyespot patterns in caterpillars. Japanese Journal of Entomology, 48, 1-5.

Sloan, S. A., Zimmerman, J. K. \& Sabat, A. M. 2006. Phenology of Plumeria alba and its Herbivores in a Tropical Dry Forest. Biotropica, 39, 195-201.

\section{Smith, M. A., Rodriguez, J. J., Whitfield, J. B., Deans, A. R., Janzen, D. H.,}

Hallwachs, W. \& Hebert, P. D. N. 2008. Extreme diversity of tropical parasitoid wasps exposed by iterative integration of natural history, DNA barcoding, morphology, and collections. Proceedings of the National Academy of Sciences of the United States of America, 105, 12359-64.

Speed, M. P. 1993. Muellerian mimicry and the psychology of predation. Animal behaviour, 45, 571-580.

Speed, M. \& Ruxton, G. D. 2010. Imperfect Batesian mimicry and the conspicuousness costs of mimetic resemblance. The American Naturalist, 176, E1-14.

Speed, M. P. \& Turner, J. R. G. 1999. Learning and memory in mimicry: II. Do we understand the mimicry spectrum? Biological Journal of the Linnean Society, 67, 281312.

Speed, M., Kelly, D. J., Davidson, A. M. \& Ruxton, G. D. 2004. Countershading enhances crypsis with some bird species but not others. Behavioral Ecology, 16, 327334.

Srygley, R. B. 1999. Incorporating motion into investigations of mimicry. Evolutionary Ecology, 13, 691-708.

Srygley, R. B. 2004. The aerodynamic costs of warning signals in palatable mimetic butterflies and their distasteful models. Proceedings. Biological sciences / The Royal Society, 271, 589-94.

Stankowich, T. \& Caro, T. 2009. Evolution of weaponry in female bovids. Proceedings. Biological sciences / The Royal Society, 276, 4329-34.

Stevens, M. 2005. The role of eyespots as anti-predator mechanisms, principally demonstrated in the Lepidoptera. Biological Reviews, 80, 573-88.

Stevens, M. \& Merilaita, S. 2009. Animal camouflage: current issues and new perspectives. Philosophical transactions of the Royal Society of London B: Biological sciences, 364, 423-7. 
Stevens, M. \& Ruxton, G. D. 2014. Do animal eyespots really mimic eyes? Current Zoology, 60, 26-36.

Stevens, M., Hopkins, E., Hinde, W., Adcock, A., Connolly, Y., Troscianko, T. \& Cuthill, I. C. 2007. Field experiments on the effectiveness of "eyespots" as predator deterrents. Animal Behaviour, 74, 1215-1227.

Stevens, M., Hardman, C. J. \& Stubbins, C. L. 2008a. Conspicuousness, not eye mimicry, makes "eyespots" effective antipredator signals. Behavioral Ecology, 19, 525531.

Stevens, M., Hardman, C. J. \& Stubbins, C. L. 2008b. Conspicuousness, not eye mimicry, makes "eyespots" effective antipredator signals. Behavioral Ecology, 19, 525531.

Stevens, M., Stubbins, C. L. \& Hardman, C. J. 2008c. The anti-predator function of "eyespots" on camouflaged and conspicuous prey. Behavioral Ecology and Sociobiology, 62, 1787-1793.

Takagi, M., Hirose, Y. \& Yamasaki, M. 1995. Antipredation defense in Papilio larvae: effective or not? In: Swallowtail butterflies: their ecology and evolutionary biology, pp. 85-92. Gainesville, FL: Scientific Publishers.

Taylor, C. H., Gilbert, F. \& Reader, T. 2013. Distance transform: a tool for the study of animal colour patterns. Methods in Ecology and Evolution, 4, 771-781.

Therneau, T. 2014. A package for survival analysis in S: R package version 2.37-7.

Valkonen, J. K., Nokelainen, O. \& Mappes, J. 2011a. Antipredatory function of head shape for vipers and their mimics. PloS one, 6, e22272.

Valkonen, J., Niskanen, M., Björklund, M. \& Mappes, J. 2011b. Disruption or aposematism? Significance of dorsal zigzag pattern of European vipers. Evolutionary Ecology, 25, 1047-1063.

Vallin, A., Jakobsson, S., Lind, J. \& Wiklund, C. 2005. Prey survival by predator intimidation: an experimental study of peacock butterfly defence against blue tits. Proceedings of the Royal Society B: Biological Sciences, 272, 1203-7.

Vallin, A., Jakobsson, S., Lind, J. \& Wiklund, C. 2006. Crypsis versus intimidationanti-predation defence in three closely related butterflies. Behavioral Ecology and Sociobiology, 59, 455-459.

Vallin, A., Dimitrova, M., Kodandaramaiah, U. \& Merilaita, S. 2011. Deflective effect and the effect of prey detectability on anti-predator function of eyespots. Behavioral Ecology and Sociobiology, 65, 1629-1636. 
Wagner, D. L. 2005. Caterpillars of Eastern North America. Princeton, NJ: Princeton University Press.

Waldbauer, G. 1970. Mimicry of hymenopteran antennae by Syrphidae. Psyche, 77, 4549.

Wallace, A. R. 1889. Darwinism: an exposition of the theory of natural selection with some of its applicaitons, . London: Macmillan and Co.

Webster, R. J., Callahan, A., Godin, J.-G. J. \& Sherratt, T. N. 2009. Behaviourally mediated crypsis in two nocturnal moths with contrasting appearance. Philosophical transactions of the Royal Society of London. Series B, Biological sciences, 364, 503-510.

Weismann, A. 1882. Studies in the theory of descent: the origin of the markings of caterpillars. London: Sampson Low, Marston, Searle, \& Rivington.

Werner, Y. 1985. Similarities of the colubrid snakes Spalerosophis and Pythonodipsas to vipers: an additional hypothesis. Copeia, 1985, 266-268.

Werner, Y. 1986. Evolutioanry implications of occasional (non-mimetic) behavioural triangulation of the head in snakes (Coluber rhodorhachis and Malpolon monspessulanus). The Snake, 18, 37-41.

Werner, Y. \& Frankenberg, E. 1982. Head triangulation in two colubrine snakes: probable behavioural reinforcement of batesian mimicry. Israel journal of zoology, 31, $137-150$.

Winterton, S. L., Guek, H. P. \& Brooks, S. J. 2012. A charismatic new species of green lacewing discovered in Malaysia (Neuroptera, Chrysopidae): the confluence of citizen scientist, online image database and cybertaxonomy. ZooKeys, 11, 1-11.

Wüster, W., Allum, C. S. E., Bjargardóttir, I. B., Bailey, K. L., Dawson, K. J., Guenioui, J., Lewis, J., McGurk, J., Moore, A. G., Niskanen, M. \& Pollard, C. P. 2004. Do aposematism and Batesian mimicry require bright colours? A test, using European viper markings. Proceedings. Biological sciences / The Royal Society, 271, 2495-9.

Xu, S., Schlüter, P. M. \& Schiestl, F. P. 2012. Pollinator-Driven Speciation in Sexually Deceptive Orchids. International Journal of Ecology, 2012, 1-9.

Zelditch, M., Swiderski, D. L., Sheets, H. D. \& Fink, W. L. 2004. Geometric morphometrics for biologists: a primer. San Diego: Elsevier Academic Press. 